\title{
Context-Aware Algorithms for Sleep Apnea Monitoring and Sensor Acceptance Using Unobtrusive Pressure Sensors Arrays
}

by

Daphné Townsend B.Sc.E., M.A.Sc.E.

A thesis submitted to the Faculty of Graduate and Postdoctoral Affairs in partial fulfillment of the requirements for the degree of

Doctor of Philosophy in Electrical and Computer Engineering

Ottawa-Carleton Institute for Electrical and Computer Engineering Department of Systems and Computer Engineering

\author{
Carleton University \\ Ottawa, Ontario, Canada, K1S 5B6
}

(C) 2012, Daphné Townsend 
Library and Archives

Canada

Published Heritage

Branch

395 Wellington Street

Ottawa ON K1A ON4

Canada
Bibliothèque et

Archives Canada

Direction du

Patrimoine de l'édition

395 , rue Wellington

Ottawa ON K1A ON4

Canada
Your file Votre référence

ISBN: 978-0-494-89311-1

Our file Notre référence

ISBN: 978-0-494-89311-1

\section{NOTICE:}

The author has granted a nonexclusive license allowing Library and Archives Canada to reproduce, publish, archive, preserve, conserve, communicate to the public by telecommunication or on the Internet, loan, distrbute and sell theses worldwide, for commercial or noncommercial purposes, in microform, paper, electronic and/or any other formats.

The author retains copyright ownership and moral rights in this thesis. Neither the thesis nor substantial extracts from it may be printed or otherwise reproduced without the author's permission.
AVIS:

L'auteur a accordé une licence non exclusive permettant à la Bibliothèque et Archives Canada de reproduire, publier, archiver, sauvegarder, conserver, transmettre au public par télécommunication ou par l'Internet, prêter, distribuer et vendre des thèses partout dans le monde, à des fins commerciales ou autres, sur support microforme, papier, électronique et/ou autres formats.

L'auteur conserve la propriété du droit d'auteur et des droits moraux qui protege cette thèse. $\mathrm{Ni}$ la thèse ni des extraits substantiels de celle-ci ne doivent être imprimés ou autrement reproduits sans son autorisation.
In compliance with the Canadian Privacy Act some supporting forms may have been removed from this thesis.

While these forms may be included in the document page count, their removal does not represent any loss of content from the thesis.
Conformément à la loi canadienne sur la protection de la vie privée, quelques formulaires secondaires ont été enlevés de cette thèse.

Bien que ces formulaires aient inclus dans la pagination, il n'y aura aucun contenu manquant. 
The undersigned recommend to the Faculty of Graduate and Postdoctoral Affairs acceptance of the thesis

\title{
Context-Aware Algorithms for Sleep Apnea Monitoring and Sensor Acceptance Using Unobtrusive Pressure Sensor Arrays
}

\author{
submitted by
}

\section{Daphne Townsend B.Sc.E., M.A.Sc.E.}

In partial fulfillment of the requirements for

the degree of Doctor of Philosophy in Electrical and Computer Engineering

Dr. Rafik Goubran, Thesis Co-supervisor

Dr. Monique Frize, Thesis Co-supervisor,

Dr. Evelyn Morin, External Examiner,

Chair, Howard Schwartz, Department of Systems and Computer Engineering

Carleton University

May 2012 


\section{Abstract}

As older adults require more health care services, solutions are needed to shift the delivery of services from hospitals and nursing facilities to clients' homes. Many sleep disorders are serious enough to interfere with activities of daily living and have significant health and social consequences. Polysomnography, while invasive and expensive, provides a quantitative assessment of sleep quality by recording physiological variables with the use of multiple face and body electrodes.

The goal of this thesis is to develop unobtrusive sleep monitoring technologies to facilitate non-invasive and home-based biomedical and preventive monitoring. This thesis presents multiple algorithms for use with unobtrusive pressure sensors in sleep monitoring. The algorithms for rollover detection, central apnea detection, sensor fusion, and delay profiling were validated with controlled experiments or with data acquired using polysomnography and unobtrusive sensors simultaneously. These algorithms incorporate context from patient-driven and data-driven sources to optimize parameters and screen patients. This thesis also presents a model of sensor acceptance as a tradeoff between privacy and autonomy.

Algorithms were developed to address the importance of central apnea and the effect of positional sleeping on apnea severity. Using data collected from the West Ottawa Sleep Center, an algorithm classified central apnea events with a sensitivity $\geq 87 \%$, specificity $\geq 99 \%$, and a Cohen's kappa value of 0.875 , which compares well with the current literature. The decision tree created using controlled experiment data identified rollovers with a sensitivity $\geq 81 \%$ and $100 \%$ specificity, which compliments existing static position detection schemes. A hypothesis was put forth that most older adults would likely not 
accept video surveillance in any situation, however frail older adults might be willing to "trade in their privacy" if it meant preventing nursing home placement. The hypothesis was accepted following a literature review of smart home monitoring sensor preferences. 


\section{Acknowledgements}

First and foremost I would like to thank my supervisors Dr. Rafik Goubran and Dr. Monique Frize for their technical insight and professional mentoring throughout this journey. Thanks to my clinical advisor, Dr. Frank Knoefel, of the Elisabeth Bruyère Research Institute for lending his clinical expertise to the work, thus paving the way for much of the research and resulting publications.

Financial support for this research was provided by Carleton University, the Ontario Graduate Scholarship program (OGS) and the National Sciences and Engineering Research Council of Canada (NSERC). Measurement instrumentation and software was provided by S4 Sensors.

Thanks to Dr. Judy Leech and Becky Walker RPSGT and for their clinical insight and technical help at the West Ottawa Sleep Center to Darlene Hebert, Emily Byron, Jodie Taylor for their administrative and technical support at Carleton University and at Elizabeth Bruyère; and to the members of the MIRG and DSP labs at Carleton University for providing a stimulating and fun environment: Megan Holtzman, Dr. Amaya Arcelus, Aneliis Tosine, Bruce Wallace, Dr. Christophe Herry, Dr. Jeff Gilchrist, Shauna Mullally and Irena Zamboni.

Thanks to my parents Carmen Deschênes and Daniel Townsend and in-laws Allison McLaren and Stan Estabrooks for their unwavering support, hot meals and endless stroller-pushing in wind, rain, sun and snow.

Lastly, heartfelt thanks go to my husband Nathan for his continuous love, support and engaging conversation, and to baby Zola. The last two years have been especially transformative and rewarding. I love them dearly. This thesis is dedicated to them. 


\section{Statement of Originality}

The statement of originality explicitly specifies the contributions made by the author, D.

Townsend, in the following publications:

[1] M. Holtzman, D. Townsend, R. Goubran and F. Knoefel, "Validation of pressure sensors for physiological monitoring in home environments," in Proc. IEEE Int. Workshop Med. Meas. Appl., 2010, pp. 38-42.

D. Townsend assisted with the data collection, conducted the load distributions and step function experiments, results interpretation and contributed to the writing.

[2] D. Townsend, M. Holtzman, R. Goubran, M. Frize and F. Knoefel, "Simulated central apnea detection using the pressure variance," in Proc. 3Ist Annu. Int. Conf. IEEE EMBS, 2009 , pp. 3917-3920.

D. Townsend and M. Holtzman equally developed the method, which was implemented by $D$. Townsend, who also led the writing.

[3] D. Townsend, M. Holtzman, R. Goubran, M. Frize and F. Knoefel, "Effect of windowing on central apnea detection," in Proc. IEEE Int. Workshop Med. Meas. Appl., 2010, pp. 117-120.

D. Townsend developed the method to use windowing to improve classification results and implemented the algorithm. D. Townsend also led the writing.

[4] D. Townsend, M. Holtzman, R. Goubran, M. Frize and F. Knoefel, "Relative Thresholding with Under Mattress Pressure Sensors to Detect Central Apnea," IEEE Trans. Instrum. Meas., vol. 60, pp. 3281-3289, Oct. 2011.

D. Townsend devised of the method to use relative thresholding for apnea detection and implemented the algorithm, and led the writing. D. Townsend was the participant in the study which collected the data, and assisted with instrumentation setup.

[5] D. Townsend, R. Goubran and F. Knoefel, "Amplitude-based central apnea screening," in Proc. IEEE Int. Symp. Med. Meas. Appl., 2011, pp. 395-398. 
D. Townsend devised of the amplitude-based method, implemented the algorithm and led the writing.

[6] D. Townsend, R. Goubran, F. Knoefel and J. Leech, "Validation of Unobtrusive Pressure Sensor Array for Central Sleep Apnea Screening," IEEE Trans. Instrum. Meas., vol. 61, Jul. 2012.

D. Townsend devised the validation process using the Cohen's kappa, implemented the algorithm to detect the apneas in the data acquired from the sleep lab and led the writing.

[7] D. Townsend, J. Leech, F. Knoefel and R. Goubran, "Clinical Investigation into the Use of an Under Mattress Pressure Sensor in the Detection of Central Apneas," 26th Annual Meeting of the Associated Professional Sleep Societies, SLEEP, 2012.

All authors contributed equally to writing the abstract.

[8] D. Townsend, R. Goubran, M. Frize and F. Knoefel, "Preliminary results on the effect of sensor position on unobtrusive rollover detection for sleep monitoring in smart homes," in Proc. 31st Annu. Int. Conf. IEEE EMBS, 2009, pp. 6135-6138.

D. Townsend designed the study, collected the data, served as participant, developed the classification system, and led the writing.

[9] D. I. Townsend, M. Holtzman, R. Goubran, M. Frize and F. Knoefel, "Measurement of Torso Movement With Delay Mapping Using an Unobtrusive Pressure-Sensor Array," IEEE Trans. Instrum. Meas., vol. 60, pp. 1751-1760, May. 2011.

D. Townsend devised of the method to map delays resulting from torso movement, implemented the algorithm, and interpreted the results.

[10] D. Townsend, R. Goubran, M. Frize and F. Knoefel, "Measuring chest movement using an array of unobtrusive pressure sensors," in Proc. IEEE Int. Workshop Med. Meas. Appl., 2010, pp. 1053-1056.

D. Townsend devised of the method to measure chest movement using data previously collected by M. Holtzman. The writing was led by D. Townsend. 
[11] M. Holtzman, D. Townsend, R. Goubran and F. Knoefel, "Breathing sensor selection during movement," in Proc. 33rd Annu. Int. Conf. IEEE EMBS, 2011, pp. 381-384.

D. Townsend contributed to writing the manuscript.

[12] D. Townsend, R. Goubran, F. Knoefel, "Time Domain Characterization of Window Length and Type on Moving Variance Signal Features", in Proc. IEEE Int. Symp. Med. Meas. Appl., 2012.

D. Townsend devised of the analysis to quantify the effects of window type and parameters on the variance signal and conducted the experiments using data collected by M. Holtzman.

[13] D. Townsend, F. Knoefel and R. Goubran, "Privacy versus autonomy: A trade off model for smart home monitoring technologies," in Proc. 33rd Annu. Int. Conf. IEEE $E M B S, 2011$, pp. 4749-4752.

D. Townsend collaborated with the authors to review retained articles and develop the model. D. Townsend proceeded with the systematic review of all articles and led the writing.

[14] D. Townsend, F. Knoefel and R. Goubran, "Home Video Monitoring: A Tradeoff Between Privacy and Autonomy," IEEE 1st Annu. Int. Conf. WIE, 2011.

D. Townsend led the writing, helped by F. Knoefel for medical advice and R. Goubran for technical advice.

Signature of thesis supervisors:

Date: 


\section{Table of Contents}

List of Tables .................................................................................................................................. xiii

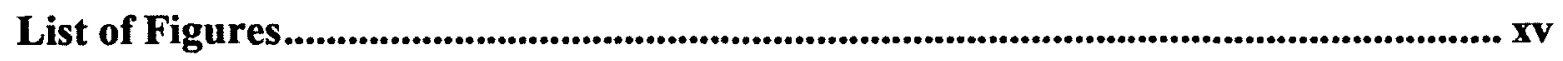

List of Abbreviations ..................................................................................................... xxi

List of Symbols ............................................................................................................................. xxiii

Chapter 1: Introduction ............................................................................................................... 1

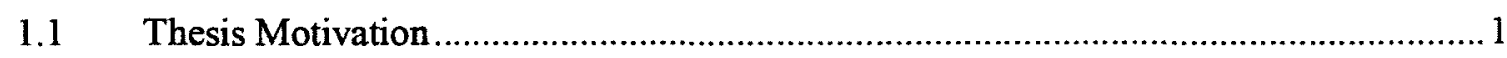

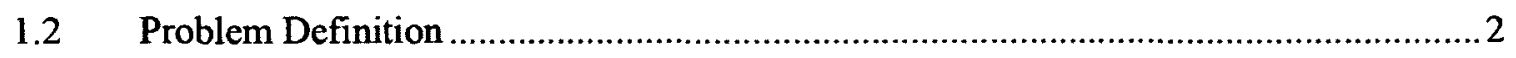

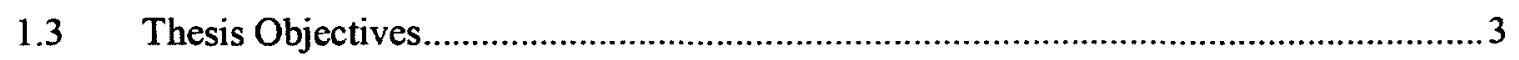

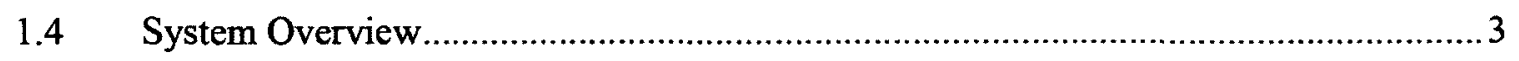

$1.5 \quad$ Research Methodology ……………...........................................................

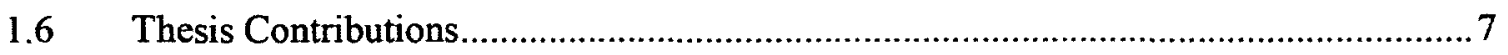

$1.7 \quad$ Document Layout ............................................................................................ 11

Chapter 2: Sleep Monitoring and Related Technologies ................................................. 12

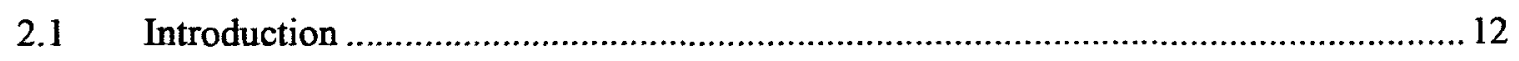

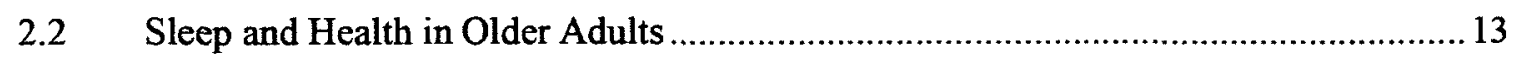

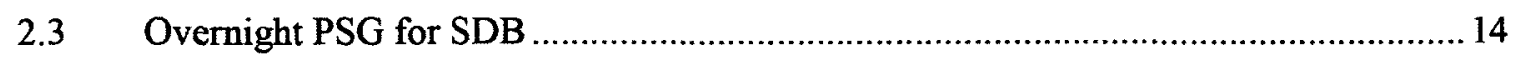

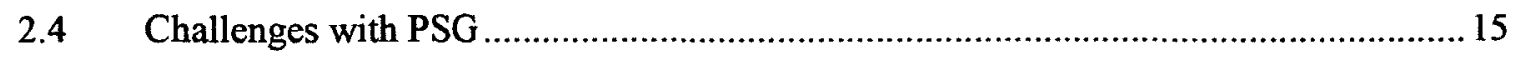

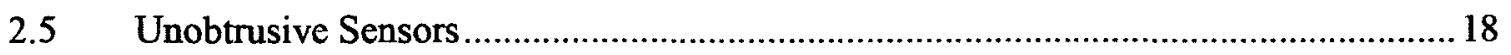

2.5.1 S4 Pressure Sensor Array ....................................................................................2

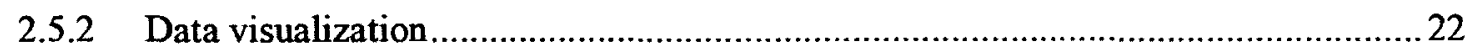

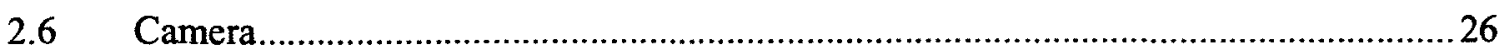

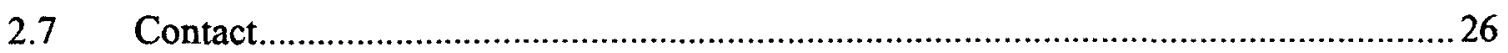

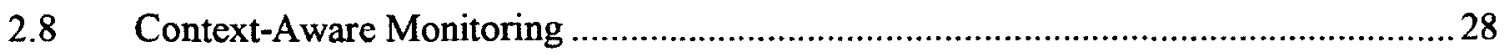


Chapter 3: Characterization of Sensor and Experimental Setup ................................ 31

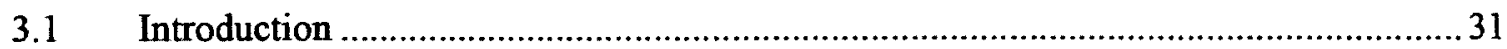

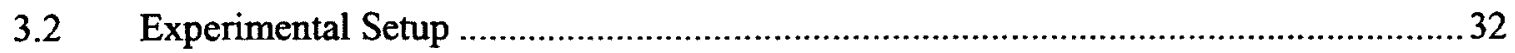

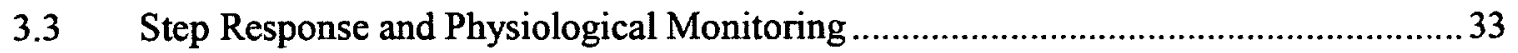

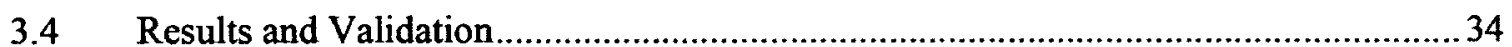

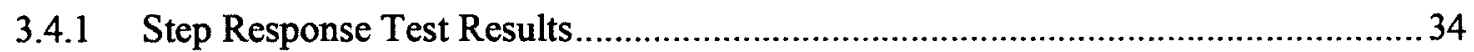

3.4.2 Physiological Monitoring Test Results .................................................................. 36

3.5 Discussion.

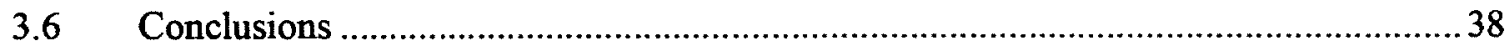

Chapter 4: Controlled Experiments for Central Apnea ............................................ 39

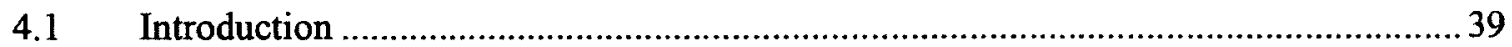

4.2 Sensor Above Mattress, Multiple Participant Trial - Controlled Experiment A ......... 40

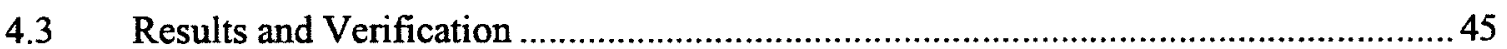

4.4 Single Participant Multiple Mattress Trial - Controlled Experiment B...................... 48

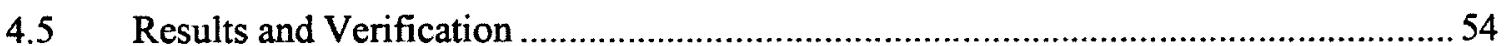

4.6 Event-Based Detection - Controlled Experiment C.......................................... 58

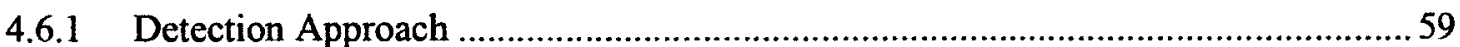

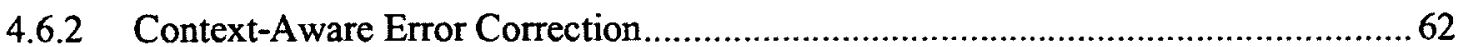

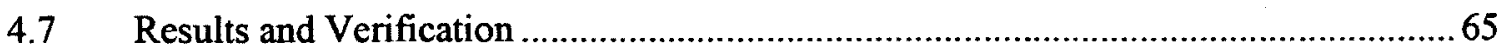

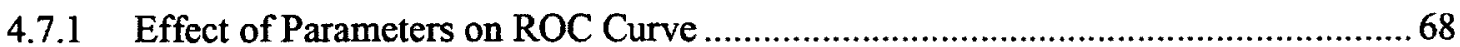

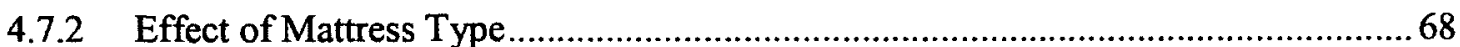

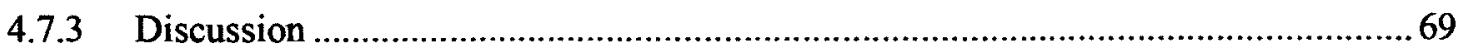

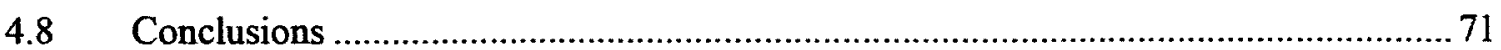

Chapter 5: Central Apnea Clinical Tests ...................................................................... 73 


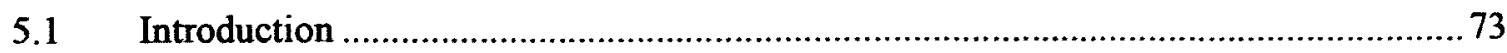

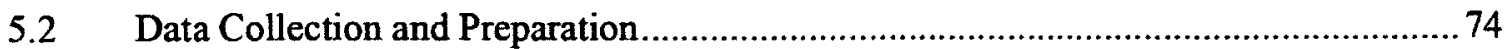

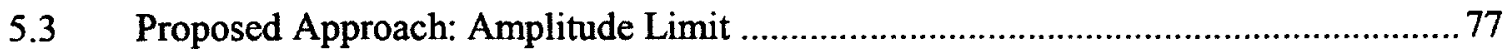

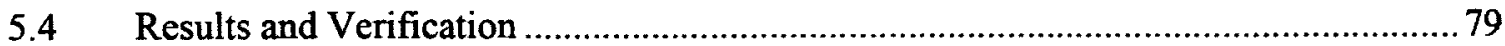

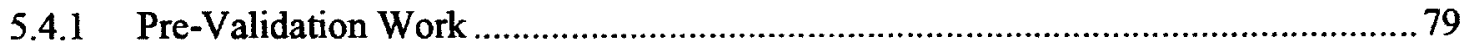

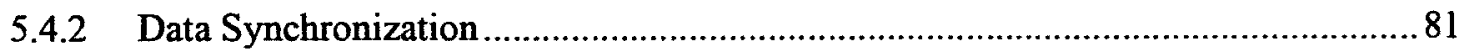

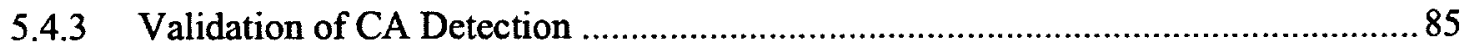

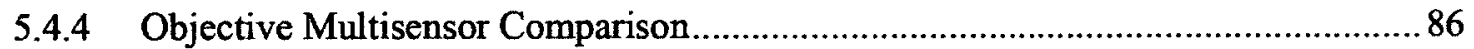

5.4.5 Effect of Desaturation Criteria on CA Scoring and Classification .......................... 88

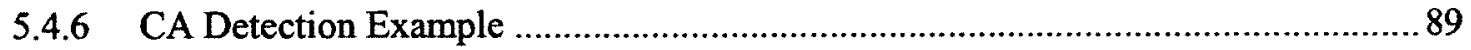

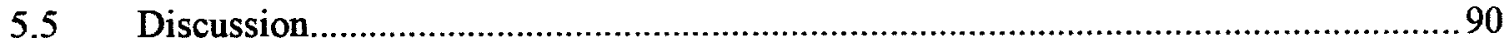

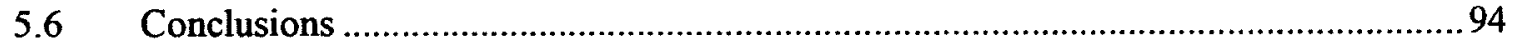

Chapter 6: Controlled Experiment for Rollover Classification ....................................95

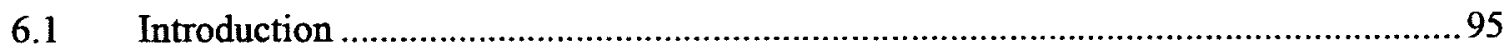

6.2 Multiple Sensor Positions - Controlled Experiment..............................................95

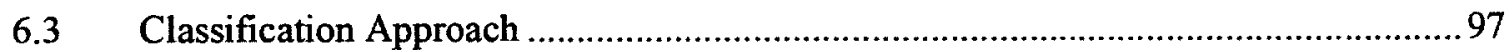

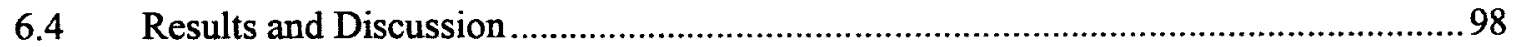

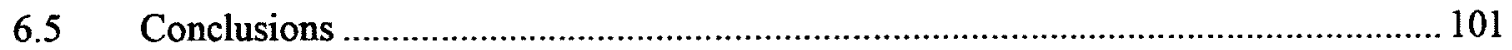

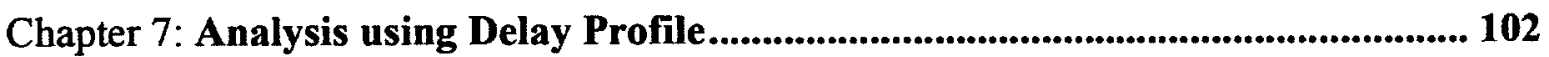

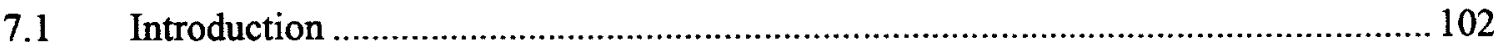

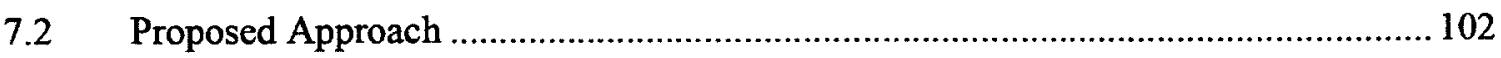

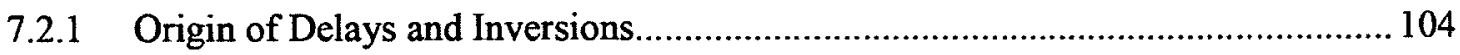

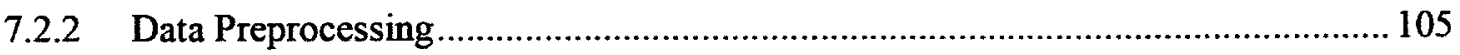

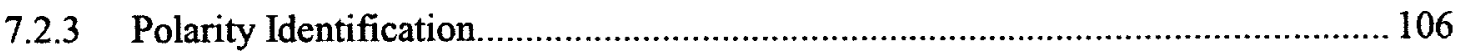

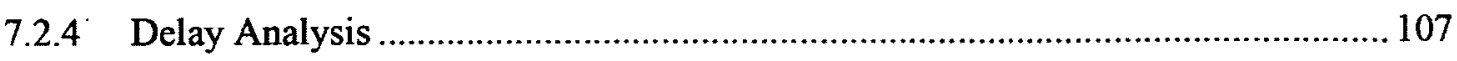

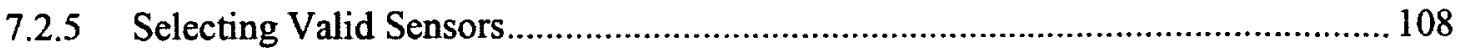




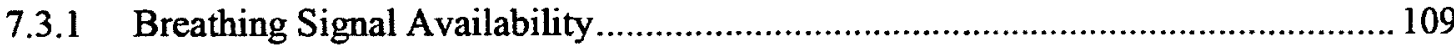

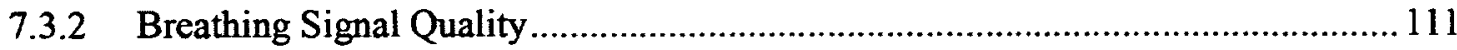

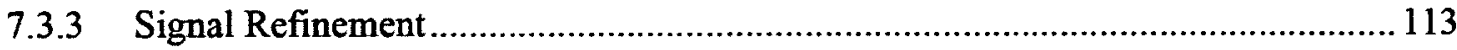

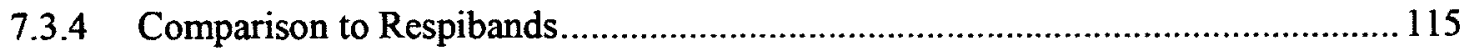

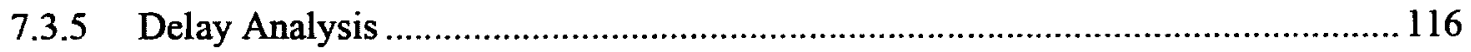

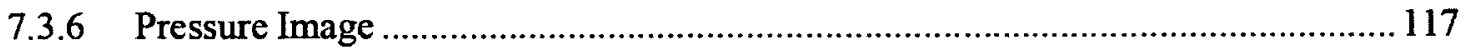

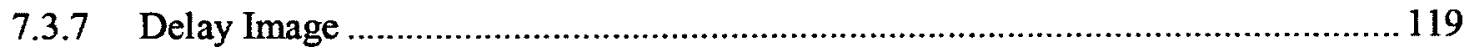

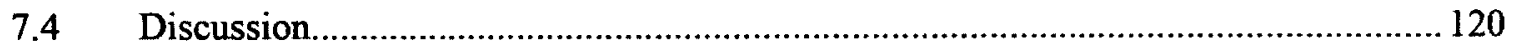

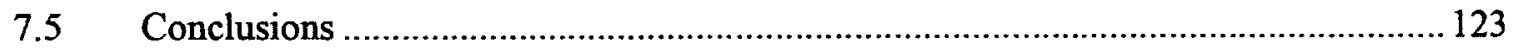

Chapter 8: Sensor Acceptance................................................................................. 124

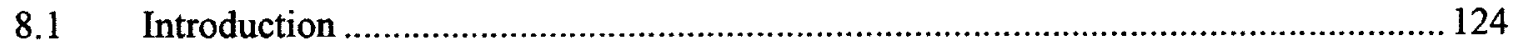

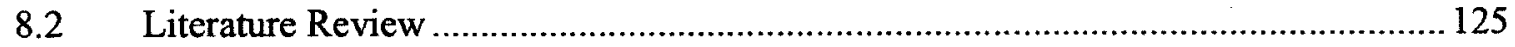

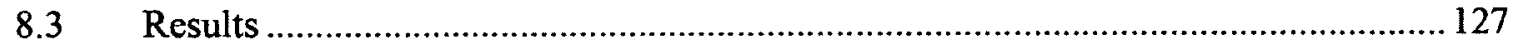

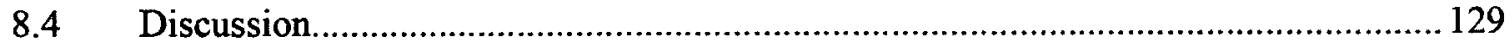

8.4.1 Tradeoff Model: Privacy versus Autonomy ....................................................... 129

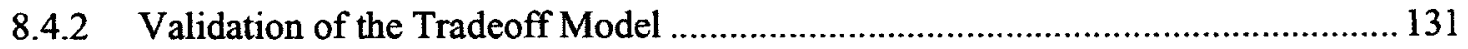

8.4.3 Challenges Comparing Focus Group and Pilot Results ................................. 132

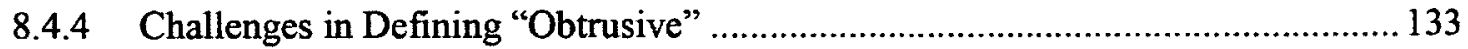

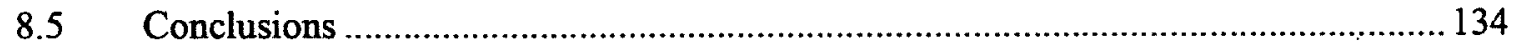

Chapter 9: Conclusions .......................................................................................................... 135

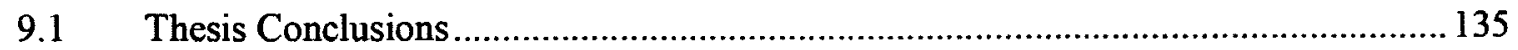

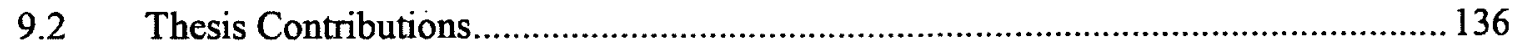

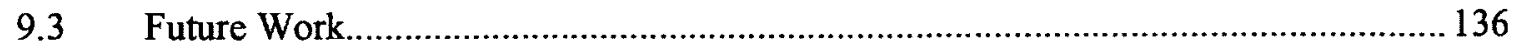

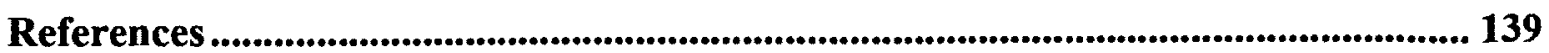




\section{List of Tables}

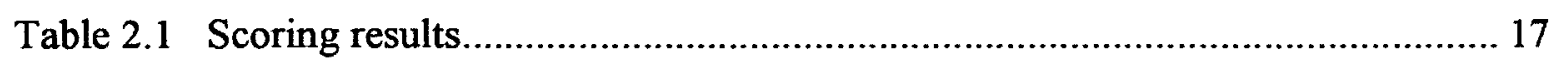

Table 3.1 Mattress types and thicknesses used in data collection .................................. 33

Table 4.1 Interparticipant CA classification results.................................................. 46

Table 4.2 CA classification results separated by position ........................................ 47

Table 4.3 Properties of recorded apneas in dataset ................................................... 49

Table 4.4 Simulated CA classification results for test set .......................................... 54

Table 4.5 Error on apnea duration estimates for both windowing methods .................. 56

Table 4.6 Position and mattress type for each CA during controlled experiment ..........59

Table 4.7 Optimal parameters determined by the training set .......................................67

Table 4.8 CA classification results for training, testing and validation set ................... 67

Table 4.9 CA classification results through algorithm evolution ............................... 72

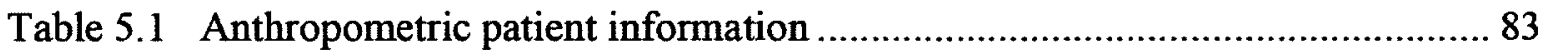

Table 5.2 CA classification results for the pressure sensor array ……......................... 86

Table 5.3 CA detection results for multiple sensor scenarios showing true positives (TP)

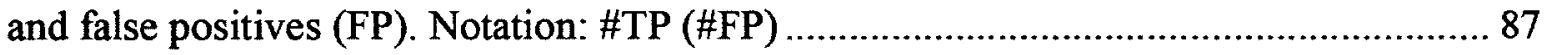

Table 5.4 Cohen's kappa coefficient for CA classification results for multiple sensor

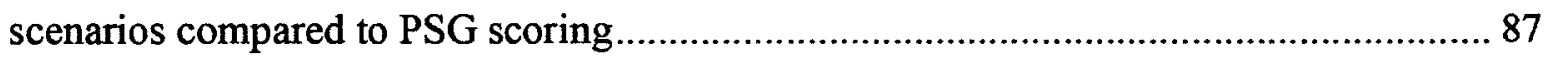

Table 5.5 ICC for CA classification for multiple sensor scenarios compared to PSG scoring

Table 5.6 Effect of including a desaturation criterion on CA Classification, Measured with Cohen's Kappa

Table 6.1 Number and type of movements for each pressure sensor position ............... 96 
Table 6.2 Extracted features for each movement. 97

Table 6.3 Intrapositional classification results for rollover detection (pruned tree)...... 99

Table 6.4 Relative feature importance for rollover detection for each positional tree 100

Table 7.1 Comparison of automated vs. manual movement detection 109

Table 7.2 Number of sensors with breathing or movement 110

Table 7.3 Epoch-based results for signal amplitude and SNR during signal combining steps 112

Table 7.4 Correlation coefficients for respibands and pressure sensor 116

Table 8.1 Type of study and number of participants for articles included in literature review 126

Table 8.2 Type of sensor presented in focus group or used in pilot 126

Table 8.3 Potential advantages to home monitoring sensors technologies as reported by participants 127

Table 8.4 Participant concerns classified in themes 127

Table 8.5 Sensor preferences as reported by participants and harmonized ranking $\ldots . .128$ 


\section{List of Figures}

Figure 1.1 System overview for validation data collection and analysis .................... 4

Figure 1.2 Traditional sensor data processing for sleep monitoring .......................... 4

Figure 1.3 Context-aware sleep monitoring.............................................................

Figure 1.4 Representation of datasets and algorithms used in chapters 4 to 7 ............. 7

Figure 2.1 Picture and technical drawing of the pressure sensitive array................... 20

Figure 2.2 Cross-sectional view of Kinotex sensor .............................................. 21

Figure 2.3 Diagram of pressure sensor array showing demarcation lines for individual

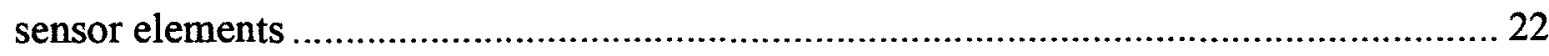

Figure 2.4 Data visualization during a rollover ................................................. 23

Figure 2.5 Time-elapsed view of selected pressure sensors during simulated apneas .... 24

Figure 2.6 Sensor output value interpreted as intensity of a pixel showing its colour changing intensity from dark to light to reflect the increase in applied pressure charted in the plot

Figure 2.7 Low resolution image of person lying on right side using normalized colour chart with lighter colours representing largest applied pressure 25

Figure 3.1 Subject in supine posture wearing two respibands ............................. 32

Figure 3.2 Step response rise time for the above mattress and below mattress pressure sensors 35

Figure 3.3 Load distribution effect in array above (top) and beneath (bottom) mattress 35

Figure 3.4 Percentage of activated sensors with respect to mattress thickness ............. 36

Figure 4.1 Block diagram for single sensor and multisensor veto approach ................. 40 
Figure 4.2 Output of two individual pressure sensors for raw data (top), data without mean load (bottom) 41

Figure 4.3 Variance for two individual pressure sensors (top), classified moving variance $(1=$ apnea, $0=$ no apnea) (middle) and number of sensors with variance $<$ threshold (bottom) 42

Figure 4.4 Histogram of $1 \mathrm{~s}$ moving variance calculated during prone breathing (top) and prone breath hold (bottom) 43

Figure 4.5 Effect of changing the number of votes on classifier performance for CA detection 45

Figure 4.6 Squaring the ROC curve by increasing number of votes 46

Figure 4.7 Model of experimental setup for controlled experiment with single sensor placed beneath mattress 48

Figure 4.8 Block diagram for windowing approach showing pre-processing, classification and windowing steps. 49

Figure 4.9 Output of two individual pressure sensors for raw data (top), data without mean load level (middle) and after filtering with $\mathrm{fc}=0.8 \mathrm{~Hz}$ (bottom). 50

Figure 4.10 Sum of the variance from all 24 pressure sensors in the array (top), classified moving variance $(1=$ apnea, $0=$ no apnea) $($ bottom $)$ 51

Figure 4.11 Chest and abdominal respiband signals and selected pressure sensor outputs during $\mathrm{CA}$ in controlled experiment 52

Figure 4.12 Respibands and classified moving variance during breathing and CA ....... 53

Figure 4.13 Windowing methods applied to classified moving variance signal 54 
Figure 4.14 Effect of window size on MCC (left) and TPR and FPR (right) on CA detection for the MA classifier

Figure 4.15 Effect of window size on MCC (left) and TPR and FPR (right) on CA detection for the WE classifier.

Figure 4.16 Calculating the apnea duration for the MA (top) and the WE (bottom) classifier 56

Figure 4.17 Comparison of apnea onset and offset for the abdomen respiband and one pressure sensor output.

Figure 4.18 Four parameter apnea model used to detect CAs in controlled experiment data 60

Figure 4.19 Four parameter apnea model applied to an apnea 60

Figure 4.20 Parametric apnea model block diagram 61

Figure 4.21 Parameter selection framework

Figure 4.22 Similarity between shallow breathing and simulated CA in respiband signal (top) and in pressure sensor array, two pressure sensor outputs shown (bottom) 63

Figure 4.23 Output of three pressure sensors (top plot). Movements, circled in black, create peaks in the variance signal (middle plot), which leads to false positives classifications (bottom plot) 64

Figure 4.24 Correct apnea detection 65

Figure 4.25 Averaged MCC results and effect of threshold d (left) and effect of $t 1$ on MCC for all d (right). Each line represents a different value of $\mathrm{tl}$ 66

Figure 4.26 Effect of $\mathrm{t} 2$ on the MCC. Each curve represents a different value for $\mathrm{t} 2$ (left) and effect of window length on MCC (right) 66 
Figure 4.27 Effect of the threshold d on the ROC curve for CA detection 68

Figure 4.28 MCC and PPV according to mattress type 69

Figure 5.1 Instrumentation setup for data collection in sleep laboratory showing sensors secured to piece of plywood located between mattress and box spring 75

Figure 5.2 Block diagram of data analysis for the amplitude limit approach 78

Figure 5.3 Central apnea model applied to moving variance signal, where ' $a$ ' is the amplitude of the variance contained within the $10 \mathrm{~s}$ sliding window. 78

Figure 5.4 Effect of the amplitude limit on the probability of CA detection for changes in window length $\mathrm{N}$ 80

Figure 5.5 Effect of mattress type on the probability of CA detection $(\mathrm{N}=1 \mathrm{~s})$ 81

Figure 5.6 PSG respiband signals during biocalibration (35 s of signals shown) 82

Figure 5.7 Output signal of selected array sensors during biocalibration of respibands 82 Figure 5.8 Central apnea $(t=23 \mathrm{~s}$ to $\mathrm{t}=40 \mathrm{~s})$ detected during a polysomnogram as captured by selected pressure sensors. 84

Figure 5.9 Central apnea in validation data as captured during the polysomnogram by the respiration band signals, air pressure sensor, and $\mathrm{Sp}_{2}$ sensor

Figure 5.10 Processed sensor array data showing correct identification of CA event (from approx. $28 \mathrm{~s}$ to $40 \mathrm{~s}$ ).

Figure 5.11 PSG signals for oronasal air pressure, and chest and abdomen respiration bands 91

Figure 5.12 Correct classification of one apnea. .92

Figure 5.13 PSG signals for one CA. 92 
Figure 6.1 Diagram of hospital bed showing sensor placement and grid layout of sensor elements

Figure 6.2 Sensor data during four rollovers (top) and approximated center of gravity signal (bottom) 98

Figure 7.1 Sensor positioning beneath volunteer and change in pressure signal shown for four sensors during normal breathing 103

Figure 7.2 Block diagram showing steps of sensor alignment, and delay correction.... 103

Figure 7.3 Recorded pressure in two adjacent sensors having inverse polarities 104

Figure 7.4 Array output showing four sensors with important properties for consideration during alignment and delay mapping. 106

Figure 7.5 Diagram of array instrumentation showing location of sensors with strongest (S), delayed (D), advanced (A) and inverted (I) signals shown in Figure 7.4 106

Figure 7.6 Output of four sensors after polarity alignment (left) and after time-alignment (right) 107

Figure 7.7 Pressure map of sensors showing movement (dark squares) for grid of $8 \times 6$ sensors (gap shown in white) 108

Figure 7.8 Number of sensors with breathing information 111

Figure 7.9 Raw sensor array data.

Figure 7.10 Evolution of signal strength during alignment algorithm 113

Figure 7.11 Number of polarity adjusted sensors per epoch 114

Figure 7.12 Epoch-based average breathing amplitude during signal alignment steps .. 115

Figure 7.13 Epoch-based SNR value during signal alignment steps 115

Figure 7.14 Delay values for participant data 117 
Figure 7.15 Aligned array data and respiband signals showing deep and regular breathing

Figure 7.16 Pressure map of breathing signal amplitude with lighter colour representing higher pressure 118

Figure 7.17 Pressure map for normalized breathing signal availability with lighter colour representing larger breathing amplitude 118

Figure 7.18 Delays between sensor outputs seen with changes in breathing depth in array data 120

Figure 7.19 Regular breathing (left) and breathing with delay (right) with lighter colour representing positive delay and darker colour representing negative delay with respect to reference sensor (shown in crosshatch) 120

Figure 8.1 Method used in selecting articles for literature review and synthesis 125 


\section{List of Abbreviations}

AASM

ADL

AHI

Avg

BCG

BMI

CA

$\mathrm{CE}$

$\mathrm{CE} \%$

$\mathrm{CP}$

CPAP

ECG

EEG

EOG

EMG

FN

FP

FPR

ICC

L

L\&P

MA

MCC

NPV

OA

OSA

$\mathrm{P}$

PPT

PPV

PSG
American Academy of Sleep Medicine

Activities of daily living

Apnea-hypopnea index

Average

Ballistocardiogram

Body-mass index

Central apnea

Corrupted epoch

Corrupted epoch percentage

Continuous physiological

Continuous positive air pressure

Electrocardiogram

Electroencephalogram

Electrooculogram

Electromyogram

False negative

False positive

False positive rate

Interclass correlation coefficient

Lateral

Location and position

Moving average windowing

Matthew's correlation coefficient

Negative predictive value

Obstructive apnea

Obstructive sleep apnea

Prone

Pulse transit time

Positive predictive value

Polysomnography 
REM

ROC

$\mathrm{S}$

SDB

Sens

$\mathrm{S}_{\mathrm{L}}$

SNR

Spe

$\mathrm{SpO}_{2}$

Stdev

TN

TP

TPR

TTR

V

WE

Rapid eye movement

Receiver operating curve

Supine

Sleep disordered breathing

Sensitivity

Length of sliding window

Signal to noise ratio

Specificity

Blood oxygen saturation

Standard deviation

True negative

True positive

True positive rate

Time to rise

Visual

Window Elimination 


\section{List of Symbols}

$\Delta$

$\kappa$

$\sigma^{2}$

$\mathrm{CoG} x$

$\mathrm{d} \%$

L

$\mathrm{N}$

$N_{\text {band }}$

$\mathrm{N}_{\text {peak }}$

Po

$\mathrm{Pc}$

Smvar

$\mathrm{SNR}_{\mathrm{db}}$

$S_{\text {peak }}$

$t_{1}$

$t_{2}$

$t_{3}$

$\mathrm{W}_{\mathrm{L}}$

$\mathrm{Wr}$
Difference between final and initial CoGx position

Cohen's kappa

$1 \mathrm{~s}$ moving variance

Center of gravity in the $\mathrm{x}$ direction

$\%$ decrease from baseline/current moving variance required to start counting $\mathrm{t} 3$ samples

Mean power loss due to placing the array below a mattress

Number of samples in moving variance window

Noise power in total respiratory frequency band

Estimated noise power in selected frequency bins

Probability of relative agreement

Probability of random agreement

Sum of moving variance signals

SNR of fused signal

Power of signal at maximal spectral peak

Time for moving variance to decrease by $\mathrm{d} \%$ to flag apnea onset

Time allotted for moving variance to return to baseline in order to flag apnea offset

Portion of apnea where moving variance $\leq \% \mathrm{~d}$ decrease, $\mathrm{t} 3$ values smaller than 10s are discarded in this experiment as they do not meet the clinical criteria for CAs discussed earlier.

Window length used for moving variance

Total pressure on each sensor array row 


\section{Chapter 1: Introduction}

\subsection{Thesis Motivation}

Many sleep disorders are serious enough to interfere with daily activities and have serious health and social implications, prompting The National Institute of Health to recommend the development of "novel noninvasive screening diagnostic methodologies that are less expensive and more widely applicable than standard full polysomnography" [15]. Doing so could help monitor the health of a greater number of individuals, some of whom may encounter roadblocks in accessing the traditional test.

Polysomnography (PSG) is a multisensor test for the diagnosis and treatment evaluation of sleep and movement disorders which involves determining the sleep stage and the presence and duration of movement and breathing events. Sleep disordered breathing (SDB) affects approximately $15 \%$ of adults [16] and many other sleep disorders related to movements are prevalent in older adults. SDB is linked to cardiovascular impairments (e.g. increased blood pressure, increased heart rate and risk of stroke), cognitive impairments (e.g.: decreased concentration, severe daytime sleepiness, headaches), and to their related effects such as an "increased risk of being involved in a deadly motor vehicle accident" [17]. There is widespread interest in addressing some of the challenges of PSG by providing sleep assessments or screenings with less obtrusive sensors, making the evaluation available in a variety of locations and enabling long-term monitoring. 
The goal of this thesis is to develop and validate algorithms for unobtrusive monitoring. This thesis presents an approach to the delivery of health care technology in nontraditional environments (e.g.: homes, smart apartments, palliative and continuing care) in part as a response to the changing context of healthcare: increasing pressures on services from the sleep laboratory, the care needs of the increasing demographic of older adults [18], and the increased recognition of the importance of sleep health as well as the aging demographics of health care providers. Central apneas in particular are the focus of this work as they cause significant health effects in the older adult population, and have a well defined morphology in many of the contact sensors used in their diagnosis. Many sensor technologies have been proposed for sleep monitoring in smart homes. Technical validation of the sensor can be performed in laboratory with PSG data; however, not all technologies are as welcomed by potential users and their acceptance must also be validated [19-21].

\subsection{Problem Definition}

Sleep monitoring for the purpose of sleep and breathing disorders is performed in a sleep laboratory, usually located in a hospital. Expanding monitoring services to homes removes the geographical barrier to access, addresses the night to night variability of certain disorders, and provides long-term monitoring, which makes epidemiological studies possible. Additionally, many of the sensors currently used to diagnose SDB are obtrusive and their complexity of use could result in low compliance and acceptance rates. This thesis begins to address some of the current challenges of traditional monitoring by presenting an alternate sensor and validating its performance against PSG for certain aspects of sleep monitoring. Context-awareness is added as a new feature to 
the traditional view on sleep monitoring by including data driven features such as body position and patient-driven features such as height and weight as algorithm parameters. Many types of sensors are available in smart homes; environmental, mounted, wearable, and embedded. Because sensor type can affect compliance and adherence to monitoring, this thesis presents a sensor acceptance model based on a literature review of smart home pilot studies and focus groups conducted with adults of age 65 and over.

\subsection{Thesis Objectives}

The main objective of this thesis is to provide elements of sleep monitoring within smart homes using a sensor that has a high likelihood of acceptance and that integrates the sensor and patient's context to improve algorithm performance. Algorithms are developed for multiple facets of sleep monitoring and are tested using controlled experiments and validated with PSG data. This thesis also presents a model of sensor acceptance which can then verify that the sensor chosen for the sleep assessment has a high chance of being accepted by older adults.

\subsection{System Overview}

The algorithms designed in this thesis make use of data collected during controlled experiments as well as during overnight PSG. In the latter case, the data was processed according to the diagram of Figure 1.1. The two data sources are the PSG sensors and the unobtrusive pressure sensor array data. The PSG signals are interpreted by an expert to determine the presence and duration of respiratory and movement events. The analysis makes use of some PSG signals and the signals from pressure sensor arrays to create single element output files which are then compared to the expert analysis. 


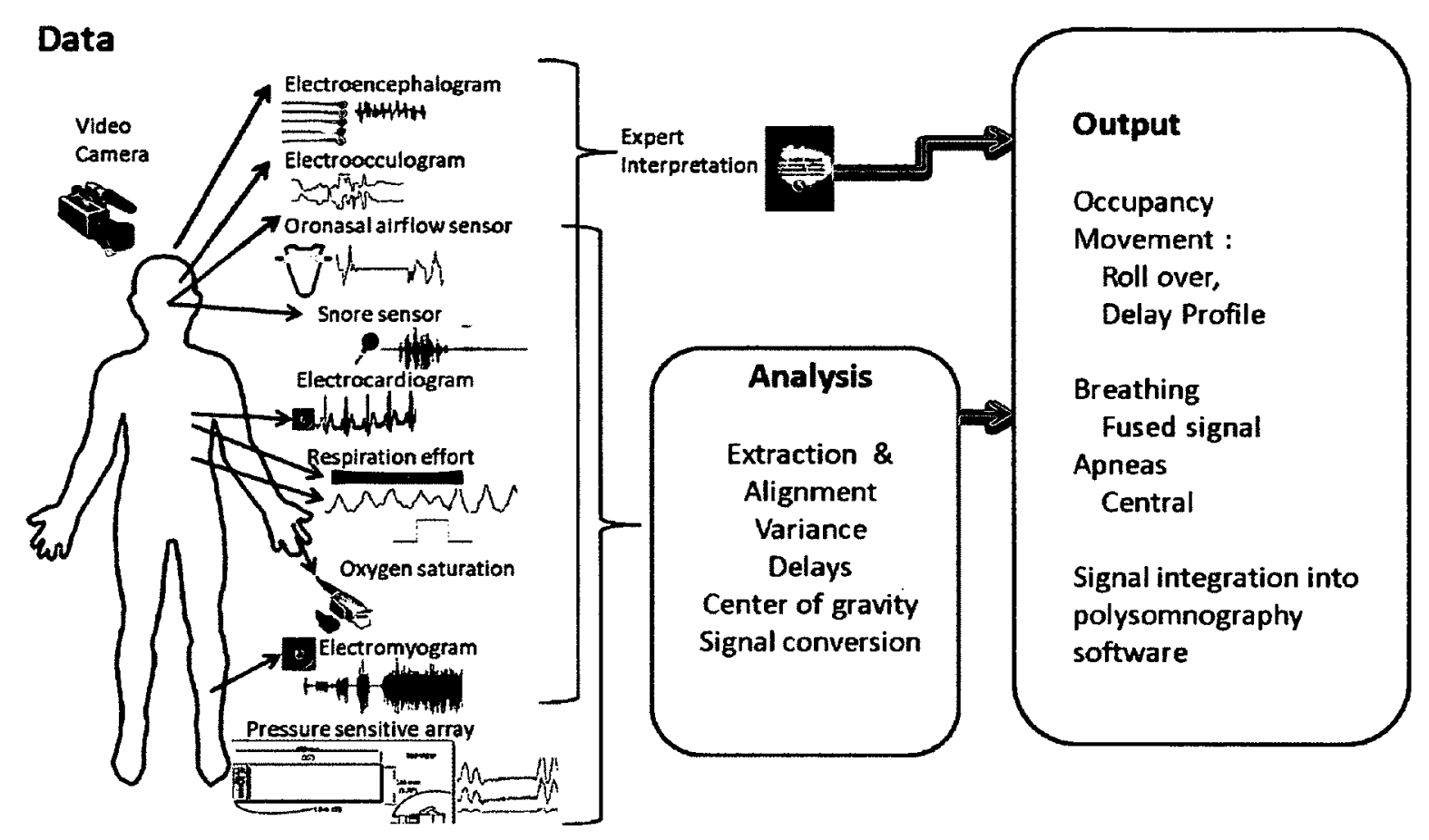

Figure 1.1 System overview for validation data collection and analysis

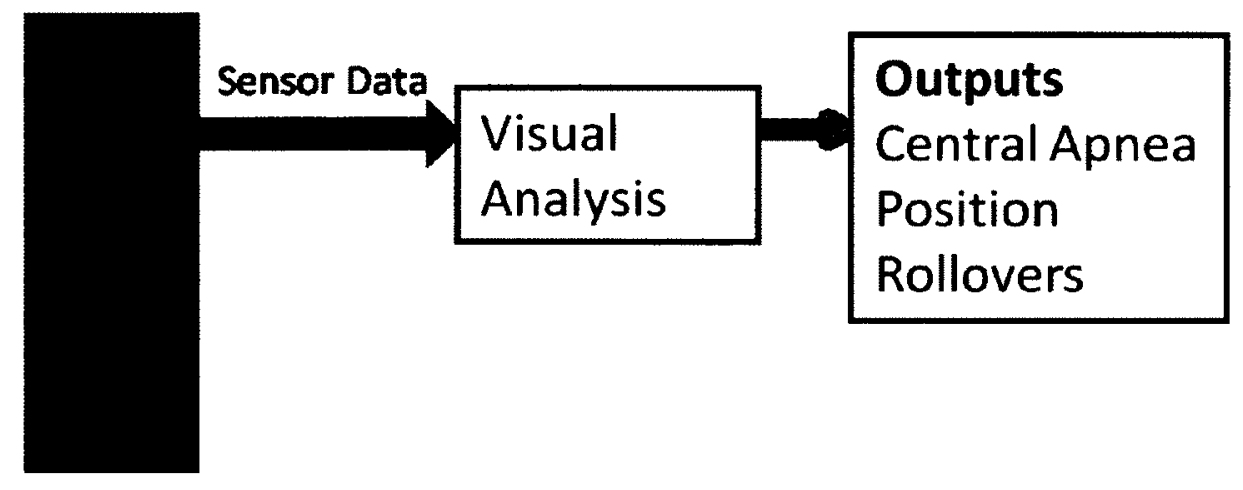

Figure 1.2 Traditional sensor data processing for sleep monitoring

Traditional algorithms for sleep monitoring rely solely on the information provided by the sensor as presented in Figure 1.2 where each sensor is subject to a visual interpretation to determine the presence of certain events. This thesis presents a contextaware algorithm as shown in Figure 1.3. The addition of information driven context (e.g.: patient's weight, height or gender) and data driven context (e.g.: information extracted by 
processing the sensor's data) used to select an algorithm are introduced. Past results can also be stored in a database and compared to current results for long-term trend analysis.
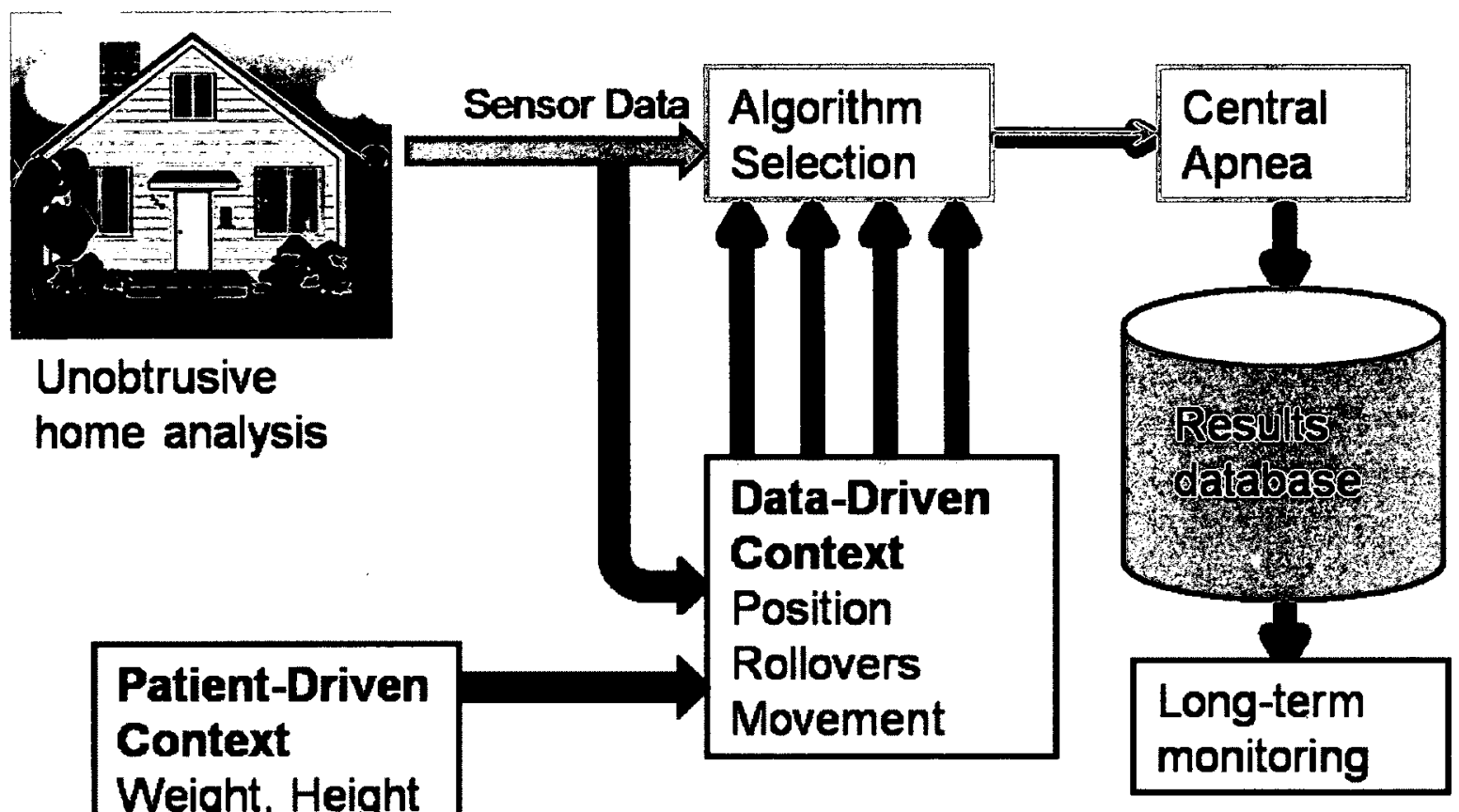

Patient-Driven

Context

Weight, Height

Mattress Type

Figure 1.3 Context-aware sleep monitoring

\subsection{Research Methodology}

The algorithms presented in this thesis are intended to monitor sleep and detect specific sleep events in a smart home occupant over long periods of time. Four controlled experiments were conducted: the first used multiple participants, the second used multiple mattresses, the third multiple sensors locations and, the fourth multiple sensors in a static location. Validation of an alternate sensor for the long-term monitoring of changes in central apnea patterns would also require long-term simultaneous PSG. Such data collection would be extremely expensive and falls outside the scope of this thesis requiring many different patients to be followed for years. Instead, controlled 
experiments were performed with healthy volunteers, and once the feasibility of central apnea (CA) detection had been established, patients scheduled to undergo a baseline PSG test were recruited to be part of the study. The data collection yielded complete datasets for over forty patients, though not all presenting with CAs. This database of complete overnight PSG records including expert interpretation and raw sensor output data for the unobtrusive pressure sensitive arrays is sufficient to demonstrate the feasibility and validity of sleep monitoring with en unobtrusive sensor array.

Figure 1.4 is a representation of the datasets and algorithms used in the experimental and validation chapters of the thesis. The 'Datasets' column describes the data and indicates whether movements or position were known. The 'Algorithms' column indicates which algorithm(s) were applied to each dataset and which context-derived variable can be used to modify the algorithm parameters to improve classification results. Below each algorithm name is listed the sections or chapter presenting the work.

Smart home pilot studies and focus groups studies discussing the use of sensors for biomedical monitoring in the home were analyzed. The sensor preferences reported by participants were used to synthesize a model of sensor acceptance. Though the model will require extensive validation with older adults in various stages of illness, and smart home occupants, it was validated by analyzing its counterparts in the literature. 
Representation of Datasets and Algorithms

Datasets

Single sensor

8 over mattress

- Single sensor under multiple mattresses

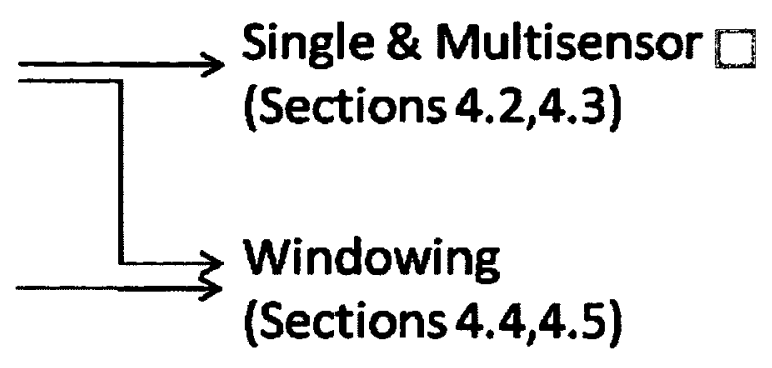

Dual sensors

under mattress

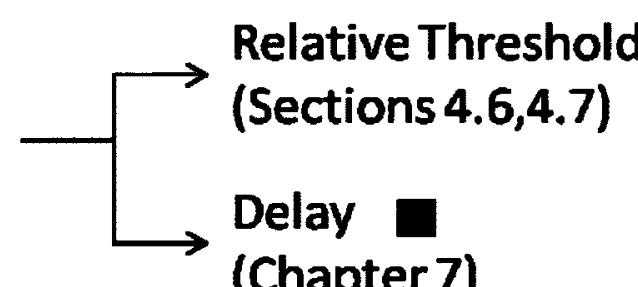

$\square$ Sleep laboratory

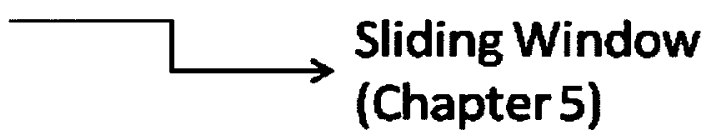

Multiple sensor positions

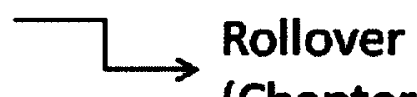

(Chapter 6)

Known in dataset:

Movements Positions

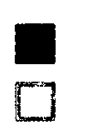

Context-Related Algorithm modifiers: Movements Positions BMI

Figure 1.4 Representation of datasets and algorithms used in chapters 4 to 7

\subsection{Thesis Contributions}

This thesis includes several contributions to the fields of unobtrusive sleep monitoring, medical instrumentation, and measurement. Most publications include both cosupervisors of this thesis Dr. Rafik Goubran, professor in the Department of Systems and 
Computer Engineering at Carleton University and Dr. Monique Frize, distinguished professor in the Department of Systems and Computer Engineering at Carleton University and professor emeritus at the School of Electrical Engineering and Computer Science at the University of Ottawa. The medical advisor for this research is Dr. Frank Knoefel, a physician specializing in geriatric rehabilitation and vice-president of medical affairs and health information at Bruyère Continuing Care in Ottawa, ON, Canada is cofounder of TAFETA, a research program focusing on the development of Technology Assisted Friendly Environment for the Third Age, where Dr. Goubran is a co-chair. Dr. Knoefel's invaluable expertise added clinical relevance and applications to most publications. Many publications resulted from collaboration with Megan Holtzman (formerly Howell Jones), a colleague and current $\mathrm{Ph}$. D. candidate in the Digital Signal Processing lab in the Department of Systems and Computer Engineering at Carleton University.

This section presents the main contributions and the resulting publications.

Contribution 1: The experimental setup for the pressure sensitive array used in this thesis was validated for physiological monitoring when placed above or below a variety of mattress types. The load distribution effect on sensor attenuation was also measured. Results of the experiments are presented in Chapter 3, with complete results published in [1].

[1] M. Holtzman, D. Townsend, R. Goubran and F. Knoefel, "Validation of pressure sensors for physiological monitoring in home environments," in Proc. IEEE Int. Workshop on Med. Meas. and Appl., 2010, pp. 38-42. 
Contribution 2: Algorithms for the detection of CAs in controlled experiment data were published in [2] and [3]. A third algorithm presenting the use of relative thresholds was published in [4] and investigated the effect of participant position as well as the effect of mattress type. These algorithms are presented in Chapter 4. A further algorithm was developed to detect the simulated CAs in both the data collected from the unobtrusive sensors and in the PSG signals. This algorithm was published in [5], and was validated in [6], where the algorithm was applied to PSG patients, and where the CA detections were compared to expert interpreters in Chapter 5. An abstract [7] presented at a medical conference discussed the clinical aspects of the study.

[2] D. Townsend, M. Holtzman, R. Goubran, M. Frize and F. Knoefel, "Simulated central apnea detection using the pressure variance," in Proc. 31st Annu. Int. Conf. IEEE EMBS, 2009 , pp. 3917-3920.

[3] D. Townsend, M. Holtzman, R. Goubran, M. Frize and F. Knoefel, "Effect of windowing on central apnea detection," in Proc. IEEE Int. Workshop on Med. Meas. Appl., 2010, pp. 117-120.

[4] D. Townsend, M. Holtzman, R. Goubran, M. Frize and F. Knoefel, "Relative Thresholding with Under Mattress Pressure Sensors to Detect Central Apnea," IEEE Trans. Instrum. Meas., vol. 60, pp. 3281-3289, Oct. 2011.

[5] D. Townsend, R. Goubran and F. Knoefel, "Amplitude-based central apnea screening," in Proc. IEEE Int. Symp.Med. Meas. Appl., 2011, pp. 395-398.

[6] D. Townsend, R. Goubran, F. Knoefel and J. Leech, "Validation of Unobtrusive Pressure Sensor Array for Central Sleep Apnea Screening," IEEE Trans. Instrum. Meas., vol. 61, July, 2012.

[7] Townsend, D. Leech, J. Knoefel, F. Goubran, R., "Clinical Investigation into the Use of an Under Mattress Pressure Sensor in the Detection of Central Apneas", 26th Annual Meeting of the Associated Professional Sleep Societies, SLEEP 2012. 
Contribution 3: The most appropriate location for the sensor under the mattress for the purpose of roll-over detection was determined. Signal features were extracted and used to identify rollovers [8]. The experiments are presented in Chapter 6.

[8] D. Townsend, R. Goubran, M. Frize and F. Knoefel, "Preliminary results on the effect of sensor position on unobtrusive rollover detection for sleep monitoring in smart homes," in Proc. 31st Annu. Int. Conf. IEEE EMBS, 2009, pp. 6135-6138.

Contribution 4: An algorithm for delay mapping was developed. The delay profile has two uses: the first is to increase the amplitude of a fused signal, while the second is to identify regions of interest with sensors having a common delay. The algorithm and its two uses were published in [9] with preliminary work presented in [10]. The derived work also included a comparison of the fused signal to respiratory inductance plethysmography (respibands), where the correlation coefficient between both sensor types was found to be significant, at $\geq 0.68$. The delay mapping technique is presented in Chapter 7. The delay profile also increases the amplitude and signal to noise ratio of fused signals. Others methods of signal combining in the presence of movement were investigated in [11] which is primarily the work of M. Holtzman, $\mathrm{Ph}$. D. candidate. Specific work on the time-domain analysis of the moving variance signal using four window types has been published [12].

[9] D. Townsend, M. Holtzman, R. Goubran, M. Frize and F. Knoefel, "Measurement of Torso Movement With Delay Mapping Using an Unobtrusive Pressure-Sensor Array," IEEE Trans. Instrum. Meas., vol. 60, pp. 1751-1760, Oct. 2011.

[10] D. Townsend, R. Goubran, M. Frize and F. Knoefel, "Measuring chest movement using an array of unobtrusive pressure sensors," in Proc. IEEE Int. Workshop Med. Meas. Appl., 2010, pp. 1053-1056. 
[11] M. Holtzman, D. Townsend, R. Goubran and F. Knoefel, "Breathing sensor selection during movement," in Proc. 33rd Annu. Int. Conf. IEEE EMBS, 2011, pp. 381-384.

[12] D. Townsend, R. Goubran, F. Knoefel, "Time Domain Characterization of Window Length and Type on Moving Variance Signal Features", in Proc IEEE Int. Symp. Med. Meas. Appl., 2012.

Contribution 5: A sensor acceptance model was created to explain an older adult's decision of whether or not to accept a monitoring technology. The model was published in [13], while the particular situation of acceptance of home video monitoring was published in [14]. The literature review and tradeoff model of privacy versus autonomy are presented in Chapter 8

[13] D. Townsend, F. Knoefel and R. Goubran, "Privacy versus autonomy: A trade off model for smart home monitoring technologies," in Proc. 33rd Annu. Int. Conf. IEEE $E M B S, 2011$, pp. 4749-4752.

[14] D. Townsend, F. Knoefel and R. Goubran, "Home Video Monitoring: A Tradeoff Between Privacy and Autonomy," IEEE 1st Annu. Int. Conf. WIE, 2011.

\subsection{Document Layout}

This thesis contains nine chapters beginning with a background review of sleep monitoring and a background review of sensor technologies, specifically for sleep monitoring in smart homes in Chapter 2. Chapter 3 presents the characterization of the sensor and experimental setup. Chapters 4 to 7 present the experimental setup and algorithms for $\mathrm{CA}$ detection and validation, rollover identification, and creation of the delay profile. Chapter 8 presents the sensor acceptance model. Thesis conclusions are presented in Chapter 9. 


\section{Chapter 2: \\ Sleep Monitoring \\ and Related \\ Technologies}

\subsection{Introduction}

This chapter reviews the relevant background information in sleep medicine and sensor technologies. The impacts of sleep and health for older adults are described in Section 2.2. The gold standard for sleep assessments is a polysomnogram, a multimodal overnight analysis. This test is described in Section 2.3 , and its challenges are presented in Section 2.4. This chapter also presents a background review of sensor technologies and the state of the art in relation to their use in non-traditional environments such as smart homes. Sensors that can be used for sleep monitoring are presented by level of intrusiveness, with a focus on those permitting some level of breath analysis, apnea detection and position detection. Section 2.5 presents unobtrusive sensors while Section 2.6 presents camera sensors. The use of contact sensors for sleep monitoring is described in Section 2.7. Context-aware sleep monitoring is presented in Section 2.8 and the chapter concludes in Section 2.9. 


\subsection{Sleep and Health in Older Adults}

Sleep medicine, a relatively young branch of medicine, studies all aspects of sleep including normal sleep, sleep disorders, sleep mechanisms, chronobiology and sleep pharmacology [22]. In the general population, the two most common sleep disorders are: insomnia and disordered breathing. Untreated, they can significantly affect the quality of life and health of sufferers. Many sleep disorders (e.g. apnea and movement disorders) are more prevalent in certain groups. Older adults ( $\geq 65$ years old) are at particular risk due to comorbidities which may impair sleep or exacerbate existing sleep disorders, which can result in excessive daytime sleepiness and cognitive impairments. A recent study of an older adult (71-87 years) population found the prevalence of SDB to be $24 \%$ [23]. Older adults also have more early morning awakenings, daytime sleepiness, depression, and increased nocturia which interfere with sleep, in addition to chronic illnesses and medication which can further fragment sleep [24], [25]. Sleep disturbances are linked to mobility problems and increased the risk of falling which is a primary cause of morbidity and mortality [25], [26]. Even in healthy older adults, sleep patterns change with age; continuous sleep periods are shorter, sleep latency increases and sleep is increasingly fragmented [24]. Disorders such as obstructive sleep apnea (OSA) are more frequent among older adults, especially men, and are related to hypertension, stroke and heart disease [25]. In older adults, sleep movements are less defined by sleep stage and more likely to lead to an awakening. Rollovers are an important sleep movement because they indicate position changes. Furthermore, positional sleeping has an effect on the severity of disorders such as apneas (both obstructive and central), snoring and Cheyne- 
Stokes respiration [27]. Rollovers do not occur during rapid eye movement (REM) sleep and may be useful for sleep staging [28].

\subsection{Overnight PSG for SDB}

PSG is the gold standard diagnostic test in sleep medicine. In the case of SDB, the patient's neurophysiologycal and cardiorespiratory parameters are recorded and interpreted from rules provided by the American Academy of Sleep Medicine (AASM), which were last revised in 2007 [29]. These rules are based primarily from the work of Rechtschaffen and Kales [30] who first devised a standard terminology, technique and scoring system for sleep stages in human subjects. The most prevalent type of SDB is sleep apnea which occurs in two major types: central and obstructive.

Central apneas (CAs) are characterized by a lack of respiratory drive. They occur frequently in premature infants due to their immature nervous system; however the majority of cases take place in older adults with heart failure when the brainstem stops sending signals to breathe in spite of increasing blood carbon dioxide levels [31]. CA is associated to congestive heart failure, and often leads to chronic fatigue, daytime sleepiness and restless sleep. The AASM recommends the following rules for the scoring of $\mathrm{CA}$, which rely on measurements from the airflow sensor, and the respiratory inductance plethysmography bands at the chest and abdomen level:

1) there is a drop in the peak thermal sensor excursion by $\geq 90 \%$ of baseline,

2) the event is $\geq 10 \mathrm{~s}$

3) $\geq 90 \%$ of the event meets criteria 1 ) and

4) absent inspiratory effort throughout the entire period of absent airflow. 
Obstructive apneas (OAs) are caused by an obstruction in the upper airway. They are characterized by a lack of airflow in spite of respiratory efforts for $\geq 10 \mathrm{~s}$. Often, breathing efforts will increase in an attempt to overcome the upper airway obstruction, resulting in a large phase offset (near 180 degrees) between the chest and abdomen respiratory bands, ending with sudden gasp as the person finally takes a breath. Much research focuses on the detection and classification of the more prevalent $\mathrm{OA}$; however CA remains an important element of SDB.

\subsection{Challenges with PSG}

Challenges with PSG include the invasiveness of the test, cost, access and a reliance on experts to interpret studies. PSG involves multiple head, face and body electrodes as well as constant monitoring through a video camera, which may not be conducive to normal sleep and for the monitoring of disorders which may vary night to night. Some patients are reluctant or unwilling to undergo testing due to the invasiveness of the test and the disruption to the normal sleeping routine. The cost of a sleep study varies greatly between countries: $800 \$$ in the United States and $450 \$$ in Australia [32]. In Canada, there is no direct cost to the patient, though there is a cost to society of an acute care hospital bed of $600 \$$ plus a consultation with a respirologist of $200 \$$. There are health and social consequences in foregoing diagnosis and treatment: studies conducted by George et al. [33] and Yoshino et al. [34] show a relationship between moderate to severe sleep apnea and motor vehicle accidents. The Canadian Medical Association's guidelines to physicians titled "Determining Medical Fitness to Operate Motor Vehicles", identifies sleep disorders as a reason for the suspension of a drivers license [35]. To this end the Ontario Health Insurance Program now subsidizes $75 \%$ of the cost of a continuous 
positive airway pressure (CPAP) device which is the standard accepted treatment for OSA [36]. The identification of older adults with problematic sleep is important, since the health benefits of treating breathing disorders have been identified. Jilek et al. for example showed the impact of treating sleep disordered breathing with a CPAP [37]. They followed a group of older apneic adults with congestive heart failure for 9 years (2002-2009) and found a significantly reduced likelihood of death in the treated group compared to the untreated group.

Access to PSG is hindered both by current capacity limits and barriers to access encountered by patients. Access can be delayed because of long wait times. These stem from the growing demand for the test partly due to the current medical interest in sleep monitoring generally and in sleep apnea in particular. Access to PSG may be difficult for older adults requiring care at night and to those providing care to a spouse at night. Additionally language barriers may exist between the technologists and some patients. Also PSG is limited to patients able to travel to hospital and willing to spend a night there. Sleep and respiration are highly disrupted in hospital in-patients as well due to sedatives and technology such as mechanical ventilators, pain, medication, noise, light and visitors. However, traditional PSG may be difficult to complete due to the frequent monitoring required to keep patients comfortable [26]. Palliative care patients also suffer from sleep fragmentation caused by illness and medication and would also benefit from a shift in the delivery of sleep monitoring services. Sleep monitoring with unobtrusive sensors addresses the availability and access barrier by bringing sleep assessments to the patient with equipment which does not interfere with any current monitors and does not prevent the patient from receiving other kinds of treatments at home or in hospital. 
A further challenge with PSG is the need for expert interpretation of the record, which is called scoring. Scoring is an interpretation of raw data signals, which requires a skilled, registered polysomnographic technologist. It is considered labor-intensive and expensive. The issue of interscorer reliability creates challenges in the comparison of scoring algorithms to human experts. Interscorer agreement is measured to quantify issues of reliability arising from the interpretive nature of the work. It is most often measured with Cohen's kappa coefficient, $\kappa$, which produces a measure of same classification beyond what would be expected by chance. In eq. (2.1) Po is the probability of relative agreement and $\mathrm{Pc}$ is the probability of random agreement. Kappa is between 1 (perfect agreement) and $[0,-1]$ depending on the distribution of the classifications, and is sensitive to misclassifications of the underrepresented class [38].

$$
\mathbf{k}=(\mathrm{Po}-\mathrm{Pc}) /(1-\mathrm{Pc})
$$

Cohen's kappa is calculated as follows for the data of Table 2.1, given two scorers interpreting the same 200 epochs for the presence of apnea. There are 20 epochs scored as apneic by both scorers and 165 epochs scored as non-apneic by both scorers. Thus, Po $=(20+165) / 200=0.925$ and $\mathrm{Pc}=(30 / 200)^{*}(25 / 200)+(170 / 200) *(175 / 200)=0.7625$. $\kappa=(0.925-0.7625) /(1-0.7625)=0.6842$.

Table 2.1 Scoring results

\begin{tabular}{|c|c|c|c|c|}
\hline & & Scorer 2 & & \\
\hline & & Asvea & N(o-Amenea & Totals \\
\hline Scorer 1 & Apnea & 20 & 10 & 30 \\
\hline & $N(0=A p$ dea & 5 & 1965 & 1 \\
\hline & Totals & 25 & 175 & 200 \\
\hline
\end{tabular}

Jensen et al. [39] studied interscorer reliability and found Cohen's Kappa of 0.87 (agreement rate $92.5 \%$ ) for 18 control patients and Cohen's Kappa of 0.73 (agreement 
rate $85.3 \%$ ) in a group of 10 Parkinson sufferers. Whitney et al. [40] measured the intraclass correlation coefficient (ICC) for respiration events in different scenarios and found that the inclusion of a desaturation criteria on the $\mathrm{SpO}_{2}$ sensor increased the ICC from 0.74 to $>0.90$. Suzuki et al. compared the classification of a single PSG record by one scorer from each of 16 different sleep laboratories in Japan [41]. They found a coefficient of variation (ratio of standard deviation divided by the mean) of 0.47 for the number of apneas detected per hour of sleep, or apnea index (AI) and that longer apneas were scored more accurately than shorter ones.

\subsection{Unobtrusive Sensors}

Unobtrusive sensors are a method of data collection which does not interfere with the subject's activities of daily living (ADL) or require any actions on the subject's behalf for the proper functioning of the sensor. Mack et al. trained a classifier for the estimation of heart rate and breathing rate averages during $30 \mathrm{~s}$ epochs and compared it to averaged rates for PSG data of 40 healthy volunteers [42]. Epochs lasting $30 \mathrm{~s}$ are selected to coordinate with the interpretation of PSG data, which is performed in $30 \mathrm{~s}$ epochs. Two pressure pads placed underneath bed sheets were adjusted to the patient's height. They obtained respiratory rate estimates within 2.10 breaths per minute compared to the respiratory inductance plethysmography bands used, and found that the discrepancy between PSG and pressure sensor averages increased with the apnea-hypopnea index (AHI) as post-apnea movement (often arousals) masked the cardiorespiratory signals.

A ballistocardiogram (BCG) signal measured with four bedpost load sensors was interpreted by Brink et al. to detect large body movements, heart rate and respiration rate during sleep [43]. Their algorithm determined whether the subject rolled towards their 
left or right, but did not specify actual body position. Similar instrumentation was used by Chung et al. to extract heart rate variability [44].

Pressure sensitive mats, which form pressure distribution pictures from an array of embedded pressure sensors, provide an enriched set of data. The static charge sensitive bed (SCSB) was presented thirty years ago to capture static charges induced by movement on a specialized membrane below a hospital mattress [45]. The physiological processes of respiration and heart rates, as well as muscular movements such as restless legs have been extracted with the SCSB. Rauhala et al. used the SCSB to detect periodic leg movements [46]. Comparing the detection accuracy to an anterior tibialis electromyogram (EMG) signal analyzed by two polysomnographers, he found an "incomplete concordance between the two methods" but a similar number of movements per hour. Watanabe et al. used a pneumatic under mattress sensor to measure movement, heartbeat, snoring and respiration for home health care monitoring [47]. They deemed the system as reliable with obtained signal to noise ratios (SNRs) ranging from 15.9 to 23.5 dB. Fox et al.[48] compared the number of epochs which contained movement with a non-contact pressure sensor array and compared it to actigraphy. They found a mean sensitivity of $79 \%$ and that the bed sensor often detected movements missed by the wrist-worn actigraphy. Beattie et al. placed a load cell under each of six bed supports for 4 PSG patients and trained a Bayes classifier with eight features, including signal variance, with 300 segments of disordered breathing and 150 of normal breathing [49]. The method correctly classified disordered breathing segments with a sensitivity of $77 \%$ and a specificity of $91 \%$. In PSG, position is assessed by infrared camera. Position was 
unobtrusively estimated by Foubert who trained a support vector machine to classify positions into six categories with an accuracy $\geq 90 \%$ using same patient data [50].

\subsubsection{S4 Pressure Sensor Array}

This section describes the fiber optic pressure sensor array which was used in the controlled experiments and for the collection of the clinical data. Figure 2.1 shows the technical drawing of the sensor reproduced with permission from S4 Sensors ${ }^{1}$. The pressure measuring sensor is constructed of nonlinear foam sandwiched in two semi-rigid plastic layers. Figure 2.2 shows the cross-sectional view of a KINOTEX ${ }^{\mathrm{TM}}$ transducer reproduced with permission from KINOTEX ${ }^{\mathrm{TM} 2}$. This sensor is similar to the one used in this thesis. Light from a light emitting diode (LED on Figure 2.2) is sent along one fiber optic cable into the foam. Light reflects on the foam and a portion of it returns along the second fiber optic cable to a photodiode.

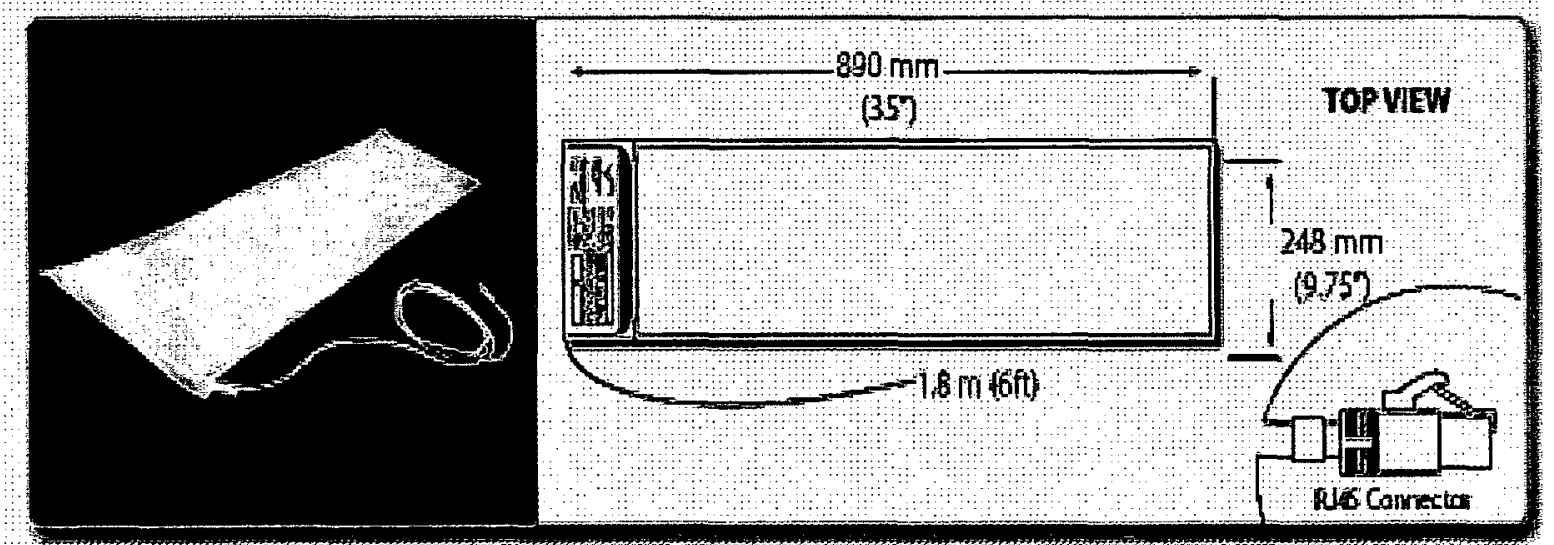

Figure 2.1 Picture and technical drawing of the pressure sensitive array

\footnotetext{
${ }^{1}$ Diagram of S4 bed occupancy sensor courtesy of S4 Sensors, www.s4sensors.com

${ }^{2}$ Diagram of Kinotex sensor, reprinted with permission from http://www.canpolar.com/principles.shtm.
} 


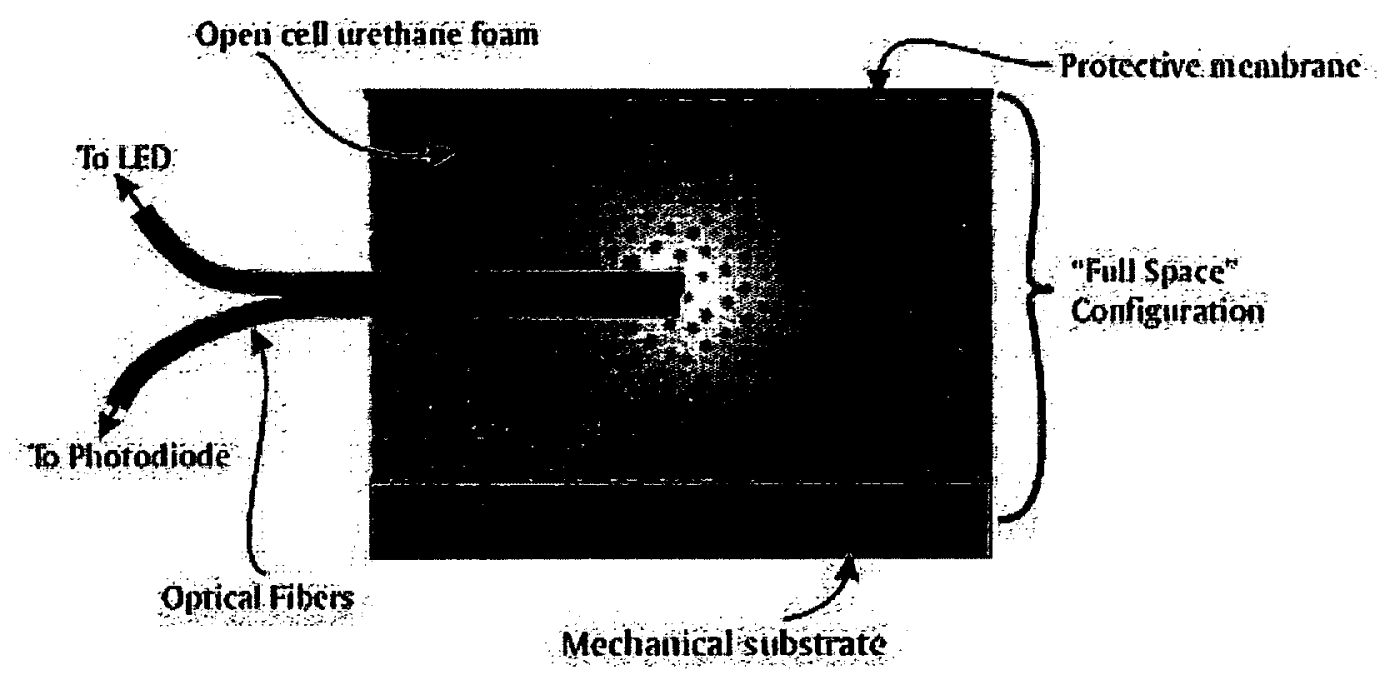

Figure 2.2 Cross-sectional view of Kinotex sensor

When pressure is applied to the protective membrane (semi-rigid plastic), the foam compresses. The increased density of the foam causes a greater quantity of light to be reflected back into the second fiber optic cable. The photodiode measures this quantity of light and generates a voltage which is converted to a pressure output value. The intensity-based output relates non-linearly to the applied pressure, and does not require calibration. The non-linear response of a similar pressure sensor has been characterized [51]. It can be placed on top of the mattress, but the rigid nature of the plastic and the sensitivity of the foam allow it to be placed underneath the mattress for use as an unobtrusive sensor which is not felt or seen by the user.

The sensor is less than $1 \mathrm{~cm}$ thick, and the pressure sensitive area of the sensor is approximately $80 \mathrm{~cm}$ by $25 \mathrm{~cm}$ and contains 24 evenly spaced sensor elements in a 8 by 3 grid as shown in Figure 2.3.The demarcation lines for individual sensor elements are not present on actual sensor, they are only presented here for illustration purposes. 


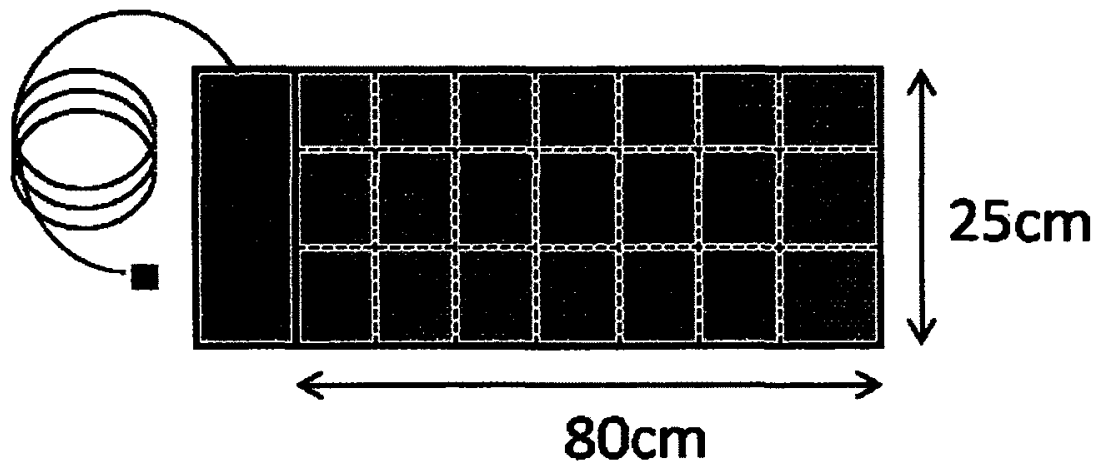

Figure 2.3 Diagram of pressure sensor array showing demarcation lines for individual sensor elements

\subsubsection{Data visualization}

The pressure sensor array data can be visualized as a pressure image which presents the pressure measured at each sensor element at a particular instance in time. The data can also be observed in a time-elapsed view where each sensor element's variations in pressure can be observed as they change over time. Figure 2.4 shows the data visualization in the pressure view and the time elapsed view for a rollover. The sensor array was located beneath the mattress and aligned with the participant's abdomen. The top row of images shows a study participant completing a rollover. The participant begins the sequence in one position (left image), begins to rollover (middle image) and completes the rollover (right image). The second row of images represents the pressure captured by the pressure sensor array according to a 'Hot' colourmap where maximum pressure is shown in white and minimal pressure in black. 


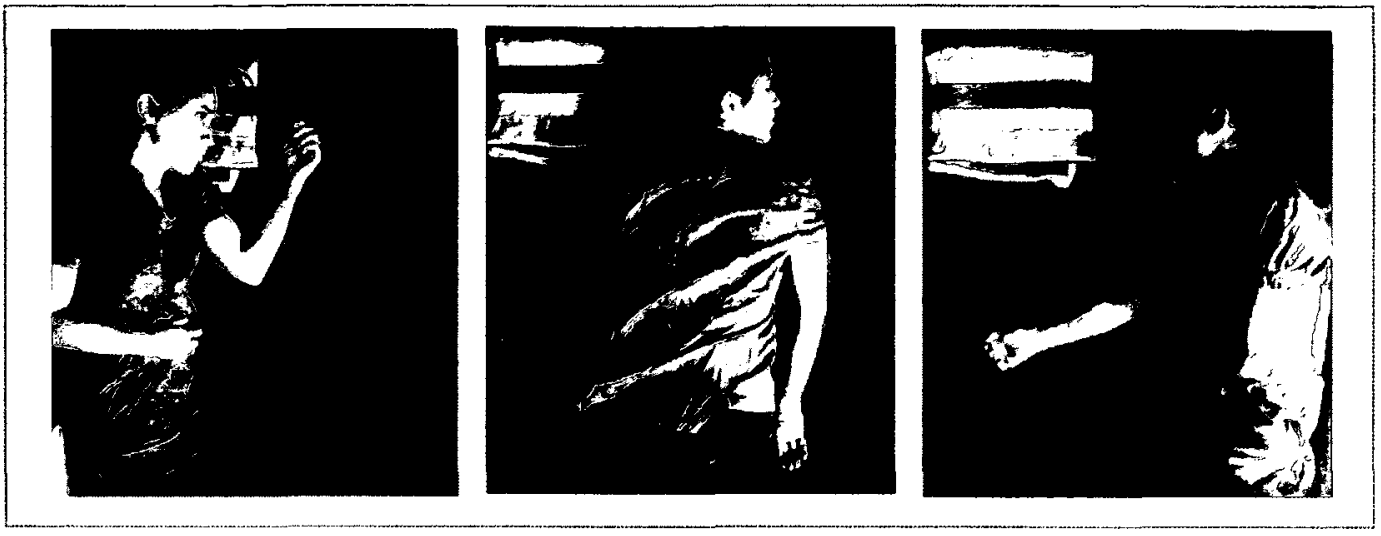

a) Top row shows participant completing rollover from left side $(t=2 \mathrm{~s})$ to prone $(t=9 \mathrm{~s})$ to right $(\mathrm{t}=18 \mathrm{~s})$ side.

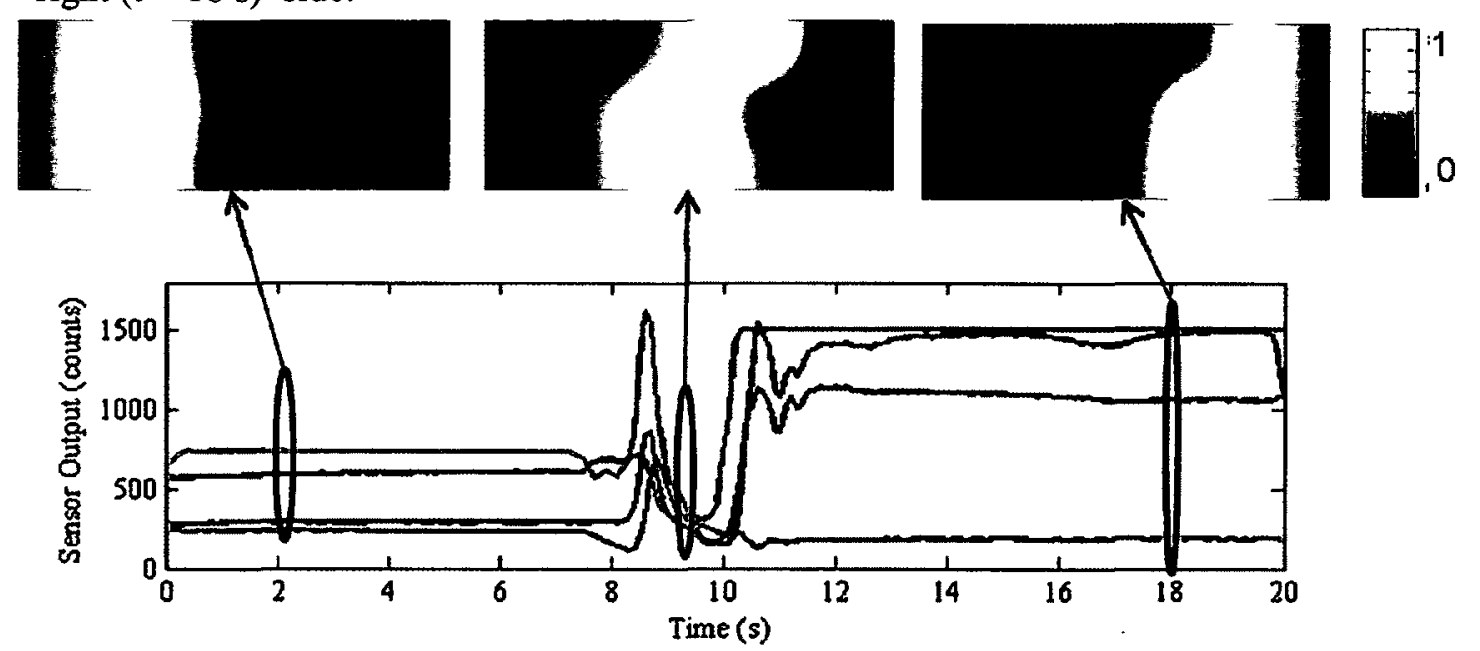

b) Second row of images shows activated sensor elements at the same three instances during the rollover, with the lighter colour indicating greater sensor activation. Bottom plot shows timeelapsed view of rollover of four sensor elements.

Figure 2.4 Data visualization during a rollover

As the rollover is completed, the most heavily weighted area of the pressure sensor shifts along with the participant from left to right. The bottom plot of Figure 2.4 presents the output of four selected sensor elements from the pressure array. Initially, the sensor element represented by the red line (top line at $t=2 \mathrm{~s}$ ) is the most heavily weighted while the others are less loaded. Upon completion of the rollover, the sensor element represented by the red line is the least heavily weighted while the purple, blue and green lines representing other sensor elements have increased in value. 
The fiber optic pressure sensor array data can be analyzed to show bed entry/exit patterns, breathing rates and identify movement onset times [52]. The individual pressure sensors collect information about the pressure detected in a local area. A pressure sensor may provide ample information about breathing motion or too little information, depending on the sensor's placement relative to the chest and abdomen of the participant. For example, sensors which are not directly under the person are less likely to contain information about physiological signals, whereas sensors located under the ribs are more likely to reveal information pertaining to breathing motion. Figure 2.5 presents a data interval containing a simulated CA (samples 300-500) and a data segment containing a simulated OA (samples 175-250) for illustrative purposes.
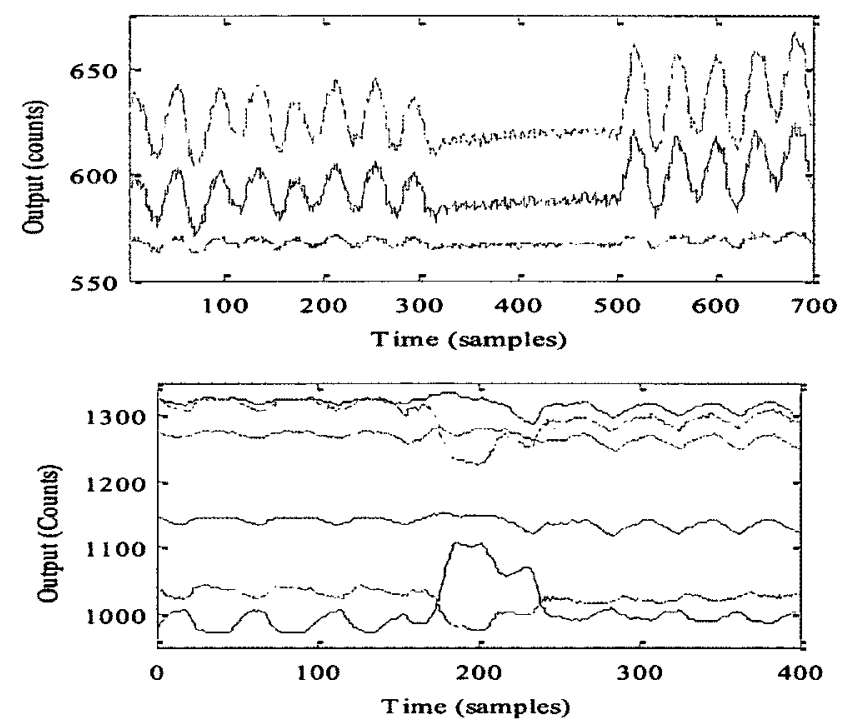

Top plot central apnea with characteristic flat signal between $t=300$ and $t=444$, and bottom shows obstructive apnea with sensor outputs going in opposite directions

Figure 2.5 Time-elapsed view of selected pressure sensors during simulated apneas

Clinically, the presence of an OA is confirmed by a phase offset in the abdominal and thoracic respiration band and by an absent or limited signal in the airflow sensor. Therefore an OA cannot be detected simply by looking for the absence of respiratory 
effort as evident in the CA. The plot of Figure 2.6 charts the output of a sensor under three different loads with increasing weight.

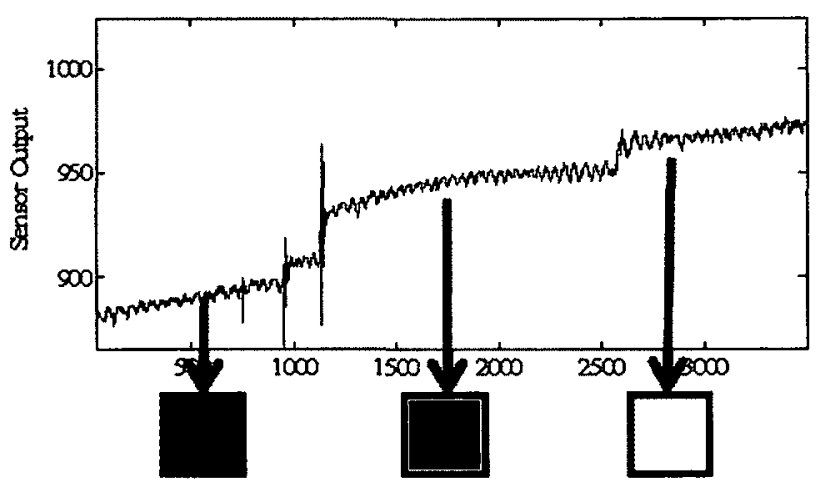

Figure 2.6 Sensor output value interpreted as intensity of a pixel showing its colour changing intensity from dark to light to reflect the increase in applied pressure charted in the plot

The three squares on the bottom row show the same data represented as an image. The sensor produces a 'pixel', with its colour changing intensity from dark to light to reflect the increase in applied pressure. In this manner, the sensor array can be interpreted as a low resolution ( 8 by 6 bit) image [9]. Stronger applied pressure results in a stronger output until saturation at 2047; at this point any increase in pressure does not result in an increased output.

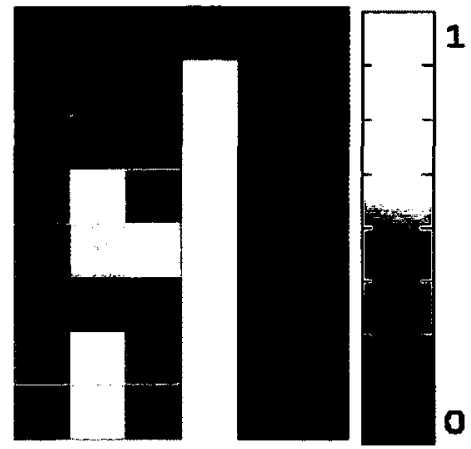

Figure 2.7 Low resolution image of person lying on right side using normalized colour chart with lighter colours representing largest applied pressure 
This technique is employed in chapter 7. Figure 2.7 shows the pressure image of a person sleeping on their right side. The pressure values are normalized to the range $[0,1]$. It is possible to discern features from the image. The white vertical pixel line is the saturated sensors at the shoulders, torso and hips while the medium intensity area on the bottom left displays the pressure from the bent knees and legs.

\subsection{Camera}

Sato et al. measured breathing motion and estimated air volume using two cameras to track a projected pattern on the bed [53]. Their multi-camera approach to respiration volume estimation obtained high correlation to volumes measured by spirometer. By exploiting the property that exhaled air is warmer than ambient air Murthy et al. successfully derived the breathing waveform for ten subjects, from the images collected by an infrared camera [54]. Ng et al. analyzed the frequency of 30 snorers with OSA and 10 snorers without to determine that a cutoff frequency of $470 \mathrm{~Hz}$ discriminated snorer types with a sensitivity of $88 \%$ and a specificity of $92 \%$ [55].

\subsection{Contact}

In PSG, contact sensors are used for all channels other than position estimation. Movement is detected with the EMG and apnea is confirmed with resistive inductance plethysmography bands (respibands), and airflow or pressure sensors. Respibands measure breathing effort through the change in chest and abdomen circumference due to the respiratory muscles and air intake, allowing inspiration and expiration to be easily distinguished. The electroencephalogram (EEG) and electrooculogram (EOG) are used to determine sleep stage. 
Alternate contact sensors have been proposed to reduce the complexity of the montage or to take the outputs of two sensors to replace a sensor. Accelerometers are used for breathing, movement and sleep-stage estimation. Wrist-worn accelerometers or actigraphs have been used since the late 1970 s to identify movements and estimate sleep stages [56]. An actigraph was used by Miwa et al. to identify rollovers who then used the frequency of rollovers (or their absence) to differentiate between two stages of sleep as determined by a commercial actigraph [28]. Bates et al. mounted accelerometers on the torso to accurately measure heart rate and breathing rate for short periods of time in static patients [57]. Morillo et al. placed the accelerometer on the throat to measure physiological parameters of PSG patients in supine position and obtained \pm 3 breaths per minute and an excellent correlation for heart rates [58]. In 2011, Sadeh reviewed the role of actigraphy in sleep monitoring [59]. He noted that in an older population there is some evidence that actigraphy can provide some indicators about individuals at risk of periodic leg movements and SDB; but that current discrimination ability is clinically insufficient. He also outlines there is some support for actigraphy to be used to measure compliance to sleep disorder treatments such as CPAP therapy.

The pulse transit time (PTT) combines an electrocardiogram (ECG) signal with a pulseoximeter signal to measure inspiratory effort in adults [60]. The work was extended to children by Pagani et al. who showed PPT to be a sensitive descriptor of respiratory effort for OSA [61]. A radial basis function was trained by Marcos et al. to classify nocturnal oximetry signals to discriminate between OSA and non-OSA patients with an accuracy of $85 \%$ in a study population of 111 OSA and 76 non-apneic patients [62]. Analyzing the respiband phase shift in breath curves to detect airway obstructions and 
OAs is performed in many automatic scoring algorithms and is a coded interpretation of the AASM guidelines.

Khandoker et al. developed automatic processing of single lead ECG to differentiate apneic form non-apneic breathing $[63,64]$, while de Chazal et al. focused on identifying OSA [65]. Analyzing airway pressure from CPAP machine to discriminate 25 OSA events from 25 CSA events was proposed by Yen et al. for patients already screened by PSG and receiving treatment via CPAP [66]. Kaniusas et al. studied the properties of an acoustical signal in 30 patients (including 10 apneic) using a microphone in a stethoscope chest piece taped near the heart. They also found that apneic snorers had a stronger presence of higher frequencies (up to $2000 \mathrm{~Hz}$ ) [67].

\subsection{Context-Aware Monitoring}

Context-aware monitoring in smart homes is a fast expanding area of research, where applications gather relevant parameters about a situation and use it to modify algorithms or interfaces which are monitoring or responding to the occupant's state. In 1994 Schilit et al. were the first to propose a definition of context-awareness as systems which adapt to "to the location of use, the collection of nearby people, hosts, and accessible devices" [68]. Robes and Kim later explain how context-awareness has expanded from state or location based to include situations which are identified by sensor [69]. A concrete example of this would be ADL recognition.

This section presents the recent literature on the incorporation of context-awareness for health-focused smart homes and presents relevant aspects of smart home infrastructure which can be included in context-aware monitoring. Still, context most often focuses on identifying the location of a person within smart home and identifying patterns of 
activity. In Kuo et al.'s camera based breath monitoring system context was limited to detecting large movements to limit computation to times that are movement-free [70]. A processing system informs the presence of movements and this context provides an on/off switch for breathing calculations. Mack et al. [42] detected outliers in the heart rate estimation and re-examines or averages them by comparison with estimates for surrounding epochs to provide context-aware error correction.

Karlen and Floreano addressed interparticipant variability in quantities of movement by using adaptable software for sleep-wake decisions made by the wearable accelerometer [71]. The device was made context aware by buzzing and requiring user feedback randomly. The software used the feedback along with processing on the movement data recorded by actigraphy to infer the sleep or wake state.

The analysis of energy usage is used for two purposes in context-aware smart homes. The first is to characterize the occupant's activity patterns and identify their location through the use of wireless power metering plugs [72]. The second is to analyze the energy uses of wireless sensors as to optimize the data transfer between sensors or between sensor and a processing node.

\subsection{Conclusions}

A new understanding about the role and importance of sleep in health combined with rapidly aging demographics presents opportunities for research and development of new approaches in monitoring. As well, challenges with the current PSG sleep test can be addressed by studying the suitability of alternate monitoring technologies and incorporating context-awareness in the developed solution. In many cases it may be more cost-effective to offer clinical services through new monitoring technologies which 
incorporate the patient's context rather than expanding services of PSG. Sensors must be selected carefully and validated to ensure they perform well in laboratory tests as well as in practice. In this thesis the sensor proposed for sleep monitoring is the unobtrusive pressure sensitive array. The inclusion of context-awareness from static and temporal data as well as stored data presents an enhancement over current methods of sleep monitoring. 


\section{Chapter 3:}

\section{Characterization of}

\section{Sensor and}

Experimental Setup

\subsection{Introduction}

Research using pressure sensors to monitor physiological signs, such as breathing and heart rate, is often performed in the context of controlled laboratory or institutional settings. The goal of this chapter is to characterize the sensor's response to different mattress types. The variability in mattress types is acknowledged to require measuring in certain applications where mattress compliance can affect sensor function and where a patient's conditions places requirements on mattresses (e.g. to prevent pressure ulcers or to facilitate bed transfers). This chapter describes the technical validation of the S4 pressure sensor used in this thesis. The chapter begins by presenting the experimental setup in Section 3.2. Section 3.3 presents controlled experiments performed to validate the use of the sensor array in diverse home environments ${ }^{3}$. Section 3.4 presents the results of the controlled experiments. The discussion of the sensor's ability to measure physiological signals is in Section 3.5 and conclusions are presented in Section 3.6.

\footnotetext{
${ }^{3}$ A significant portion of this chapter is published in [1].
} 


\subsection{Experimental Setup}

As presented in Chapter 1 this thesis focuses on unobtrusive sleep monitoring. To observe the effect of sensor placement on physiological signal capture, the responsiveness of two pressure sensor arrays were compared. One was placed on top of a mattress; where it would be in contact with a person lying down and the second was placed below the same mattress, directly under the top array using a ruler to align the sensors. Data was collected from both arrays concurrently. Figure 3.1 shows the subject in supine posture on the $35 \mathrm{~cm}$ pillow top mattress. The subject is seen wearing two respibands. One pressure sensor array is located under the top sheet directly beneath the torso of the participant and the second sensor is located underneath the mattress, directly beneath the first sensor. Each sensor array was first connected to a data acquisition box, then to the laptops using one serial connection and one USB connection.

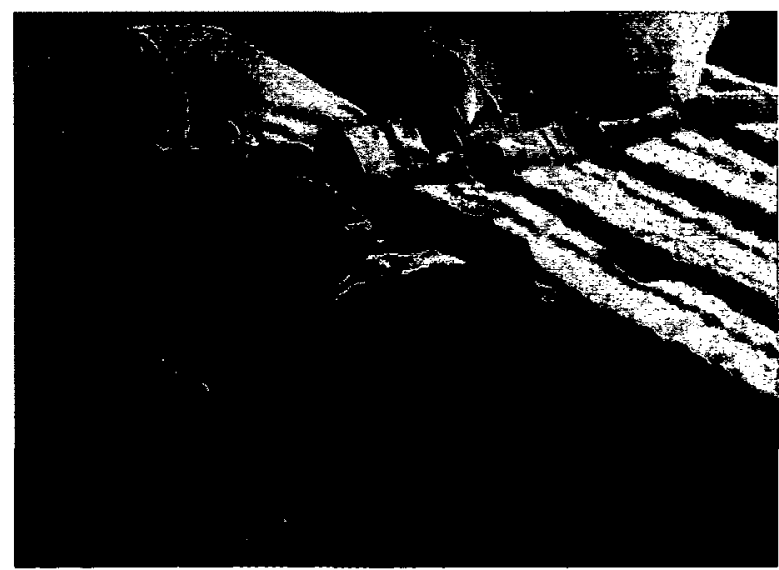

Figure 3.1 Subject in supine posture wearing two respibands 


\subsection{Step Response and Physiological Monitoring}

The measurement equipment was brought to four locations in order to collect biomedical data under a variety of scenarios to simulate the actual context of use. The nine mattresses tested ranged from $15 \mathrm{~cm}$ to $35 \mathrm{~cm}$ in thickness and were manufactured from popular materials: coil, foam, and futon. The institutional mattress was from a sleep laboratory (different from the sleep laboratory used for data collection in Chapter 5). Often, higher end foam mattresses are made of latex rubber foam, while less expensive foam mattresses are made with polyethylene foam. As such, both latex and polyurethane mattresses were used in this data collection. Table 3.1 which details the mattresses used. In that table, the latex mattress is indicated with a superscript $L$.

Table 3.1 Mattress types and thicknesses used in data collection

\begin{tabular}{|lllll|}
\hline$\#$ & Type & $\begin{array}{l}\text { Mattress } \\
(\mathbf{c m})\end{array}$ & $\begin{array}{l}\text { Pillow top } \\
(\mathbf{c m})\end{array}$ & Total $(\mathbf{c m})$ \\
\hline $\mathbf{1}$ & Coil & 23.0 & 12.0 & 35.0 \\
\hline $\mathbf{2}$ & Coil & 18.0 & 15.0 & 33.0 \\
\hline $\mathbf{3}$ & Coil & 18.0 & 5.0 & 23.0 \\
\hline $\mathbf{4}$ & Coil & 18.0 & 0.0 & 18.0 \\
\hline $\mathbf{5}$ & Foam & 15.2 & 0.0 & 15.2 \\
\hline $\mathbf{6}$ & Foam & 15.2 & 0.0 & 15.2 \\
\hline 7 & Foam & 15.2 & 0.0 & 15.2 \\
\hline $\mathbf{8}$ & L Foam & 15.2 & 5.0 & 20.2 \\
\hline $\mathbf{9}$ & Futon & 15.2 & 0.0 & 15.2 \\
\hline
\end{tabular}

Two tests were conducted, the first was a step response and the second was physiological monitoring. In the case of the step response, a $4.5 \mathrm{~kg}$ circular weight with a diameter of $20 \mathrm{~cm}$ was placed on the top sensor for 5 minutes. This test created the step response as well as load distribution patterns which were evaluated by comparing the number of sensors that were activated for each mat. Activation was defined as occurring when a 
sensor output rose more than $2 \%$ of its maximum possible value in response to the weight.

The second test required a subject to lay supine, prone and laterally while performing various types of breathing and limb movements to allow the observation of the respiration signal and possibly detect cardiogenic oscillations during simulated CAs. Signal power from each sensor was calculated according to the unbiased sample variance of the sensor output, omitting samples when movements occur. For each breath type, a reference signal was selected from each sensor array. The average fraction of power available from the bottom reference signal with respect to the top reference signal was calculated as the mean power loss, $L$ over all of the $S$ segments with eq. (3.1). $L$ is measured in decibels. The power loss per $\mathrm{cm}$, Loss $_{\mathrm{cm}}$ was also calculated by dividing $\mathrm{L}$ by the total mattress thickness.

$$
\mathrm{L}=10 \log \left(\frac{1}{s} \sum_{s=0}^{\mathrm{s}-1} \frac{\max \left(\mathrm{P}_{\text {above }}\right)}{\max \left(\mathrm{P}_{\text {below }}\right)}\right)
$$

\subsection{Results and Validation}

\subsubsection{Step Response Test Results}

Figure 3.2 presents a plot of the step response during the first $300 \mathrm{~s}$ after the weight drop. The step response of the sensor placed above the mattress is shown as the red line and the step response of the sensor placed under the mattress is shown as a blue dashed line. Initial reaction time is similar, with both arrays reaching the $80 \%$ mark within a few samples. On average, the under mattress sensors took a bit more time to reach the $98 \%$ line, then overtook the over mattress sensors. Many of the signals were still slowly drifting at the end of the recorded time. 


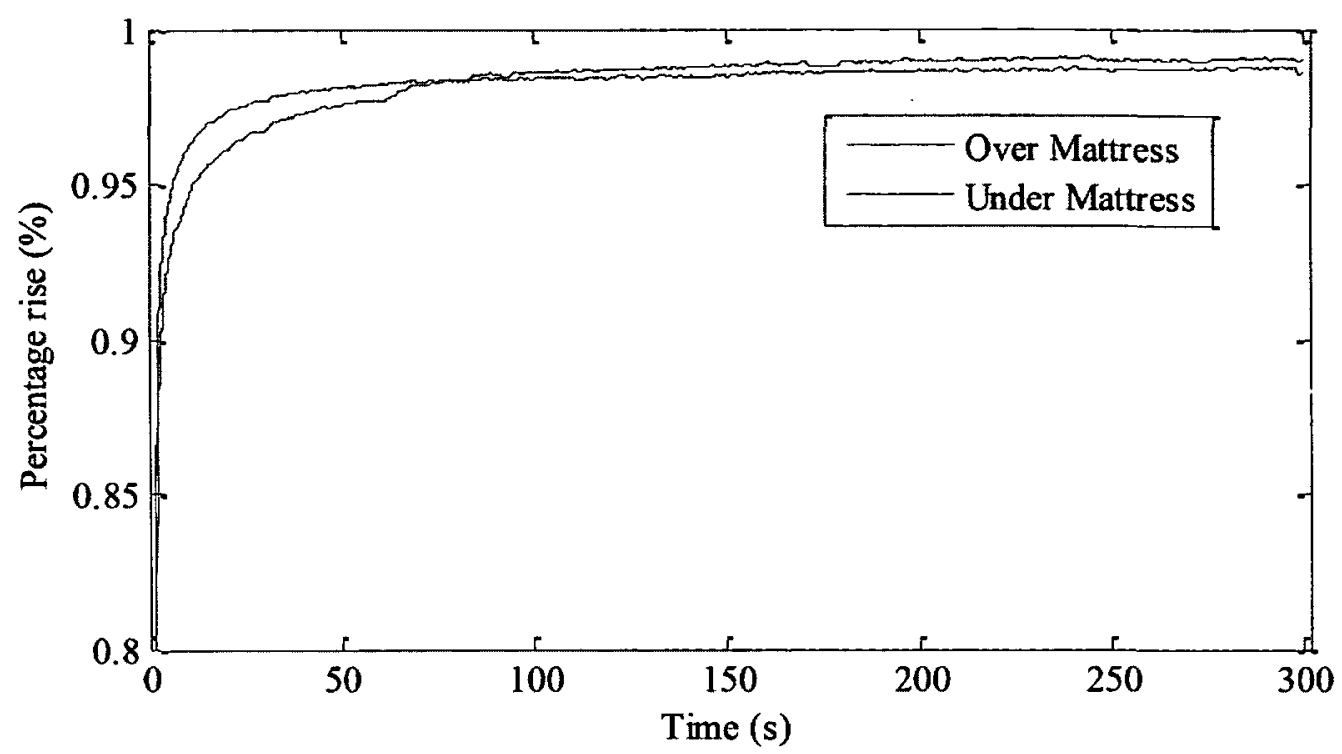

Figure 3.2 Step response rise time for the above mattress and below mattress pressure sensors

Signal drift is accommodated in data pre-processing by removing a $30 \mathrm{~s}$ moving average signal. The weight pressure captured by the two arrays during step response testing is exemplified in the interpolated pressure images of Figure 3.3, with both clearly depicting the presence of the weight. The sensor array below the mattress exhibits the effects of load distribution.
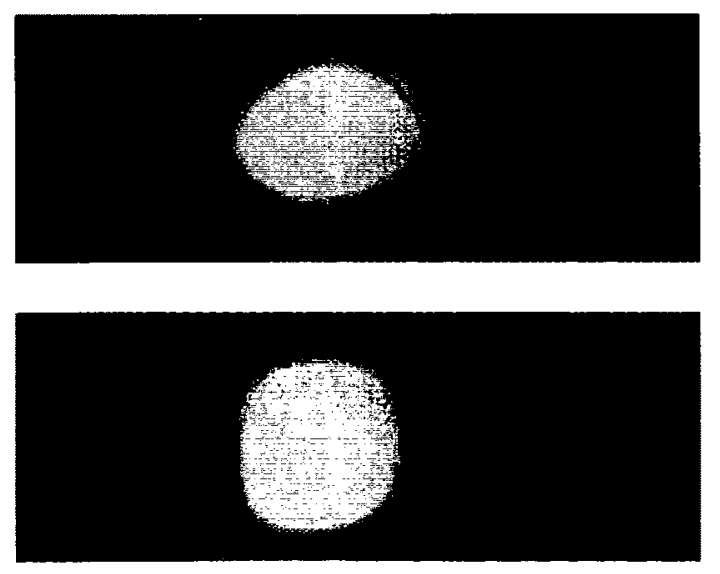

Figure 3.3 Load distribution effect in array above (top) and beneath (bottom) mattress 
Figure 3.4 plots the percentage of sensors in the array activated by the weight with respect to mattress thickness for both arrays. The number of activated sensors below the mattress increased with the mattress thickness though the mattress material may have had some effect as well. Interestingly, the thickness of the mattress has the inverse effect on the sensor pad above the mattress. The dashed line in Figure 3.4 indicates that increased mattress thickness resulted in fewer sensor activations. This may be because greater cushioning provided by thicker mattresses yields less stiffness between adjacent sensors, leading to better localization.

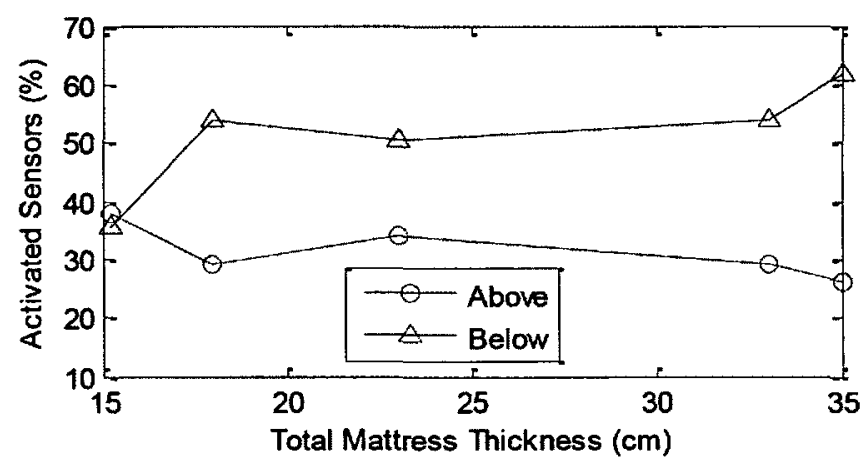

Figure 3.4 Percentage of activated sensors with respect to mattress thickness

\subsubsection{Physiological Monitoring Test Results}

M. Holtzman, the primary author in [1] conducted the analysis of the physiological monitoring test which revealed that as mattress thickness increases, generally more power is lost. On average, $69 \pm 12 \%$ of the sensors detected the respiratory signal above the mattress, and $22 \pm 10 \%$ below. In all instances, at least two sensors detected the respiratory signal. Chen et al reported breath detection rates between $76 \%$ and $99 \%$ when a differential pressure sensor was positioned in varying locations under a mattress; however the mattress type was not reported. [73]. The measurements for this controlled experiment show a much lower availability, but the sensors detect pressure over a smaller 
surface area and may be more localized. During simulated CAs, cardiogenic oscillations were available from at least one sensor for all mattresses and postures from above the mattress, and in $97 \%$ of cases from below. This compares closely with Chen et al.'s results [73]. Similarly, Watanabe achieved $97.6 \%$ availability of heart rate signals from below the mattress [47].

\subsection{Discussion}

The sensor array used in this work shows a signal response to many signatures of ADL such as bed entry and exit patterns, and other large and small body movements. As well, movements which are the result of normal breathing can be detected from beneath the mattress. During breath holds, the BCG signal is often available from the sensor placed below the mattress. The BCG signal was not measured during breathing as some frequencies may overlap with the breathing frequencies and overpower it. The pressure sensors itself can be adjusted to suit a particular application by adapting the sensitivity and response of the digital to analog converter in the data acquisition box, and by changing the foam contained in the array. The array however cannot detect some of the signals measured in polysomnography such as the EEG, EOG and airflow as these signals require contact sensors. Tanimoto et al. studied the properties of three pressure relieving mattresses [74]. The foam mattresses aim to evenly distribute pressure over a wide area to alleviate pressure ulcers using pressure distribution of the sacro-coccygeal region with a "Tekscan "Big-Mat" pressure measurement system". Mattress properties were quantified with the intention of helping spinal cord injury patients select a mattress that is sufficiently compliant to reduce pressure-ulcers but not so compressible that it hinders a person's sit-to-stand transition. 


\subsection{Conclusions}

Sensors placed below a mattress are less obtrusive and more comfortable than those placed above the mattress while retaining the potential for physiological signal monitoring. On exchange, the often heavily muted signals may feature less distinct localized loading and require more sophisticated signal extraction algorithms. 


\section{Chapter 4: Controlled \\ Experiments for Central Apnea}

\subsection{Introduction}

As introduced in Chapter 2, and supported by the National Institute of Health's research recommendations, there is increasing interest in using alternate sensors to detect sleep apneas. The following chapter presents three controlled experiments. The first experiment in Section 4.2 verifies that the pressure sensor can detect simulated CAs when placed above the mattress. The experiment's results are presented in Section 4.3. The second experiment described in Section 4.4 sees the pressure sensor located beneath the mattress and observes the effect of including position in the presented algorithm, and the results are presented in Section 4.5. The third experiment, in Section 4.6 presents an algorithm with a relative threshold and observes the effect of participant position and mattresses on algorithm parameters and classification. This experiment's results are in Section 0. The chapter concludes in Section 4.8 . 


\subsection{Sensor Above Mattress, Multiple Participant Trial - Controlled Experiment A}

This controlled experiment validates the use of the variance signal as having discrimination ability for simulated $\mathrm{CAs}^{4}$. The pressure array, located above the mattress was connected serially to a Compaq Amada $700 \mathrm{MHz}$ laptop. Software provided by S4 Sensors recorded time stamps and data from each of the pressure sensors into a commaseparated values log file. The data was originally collected for Megan Holtzman's master's thesis [75]. Seven study volunteers between 20 and 30 years old participated in the controlled experiment. They were asked to lie quietly on their own beds, with a pressure sensor located on top of their mattress. They performed a variety of breathing patterns in prone, supine and side lying as well as body movements during the data collection. Volunteers held their breath for up to $30 \mathrm{~s}$ to simulate CAs as suggested by Watanabe et al. [47]. Apnea onset time, offset times and times and posture were logged by an observer into a time stamped log file, which was manually aligned prior to data processing. Figure 4.1 presents the block diagram. Each sensor's output was filtered through a $30 \mathrm{~s}$ moving average filter to provide a mean load level as shown in eq. (4.1).

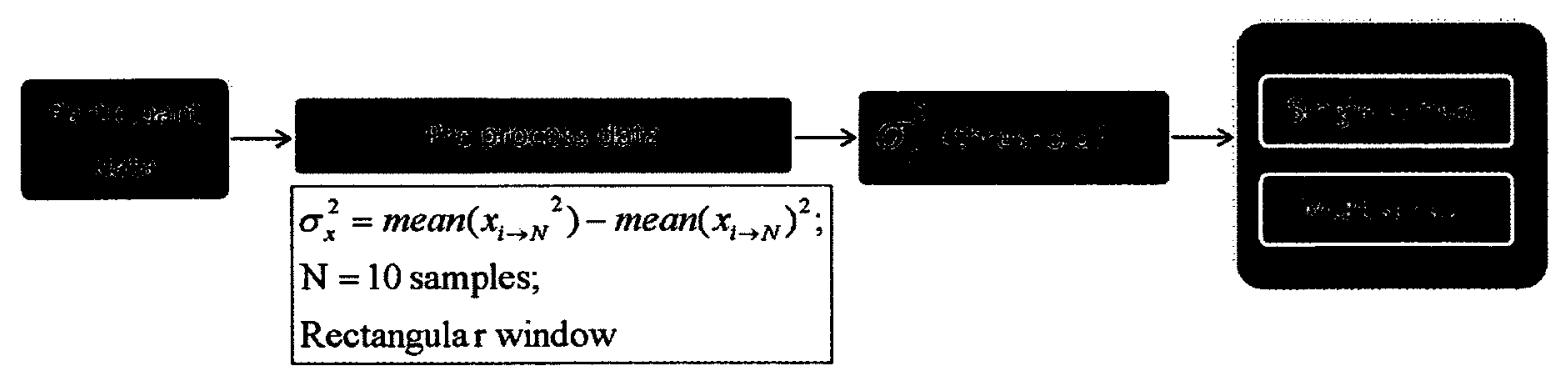

Figure 4.1 Block diagram for single sensor and multisensor veto approach

\footnotetext{
${ }^{4}$ A significant portion of Sections 4.2 and 4.3 is published in [2]
} 
$M A(i)=: x(i+N)+x(i+N-)+. .+x(i+)+.x(i)) / N s ; N s=300$

Figure 4.2 shows the output of two selected pressure sensors during the data preprocessing stage. The figure shows the data output from the acquisition box in the top plot. The bottom plot shows the raw data with the mean loading value removed. The signals are now centered at zero.

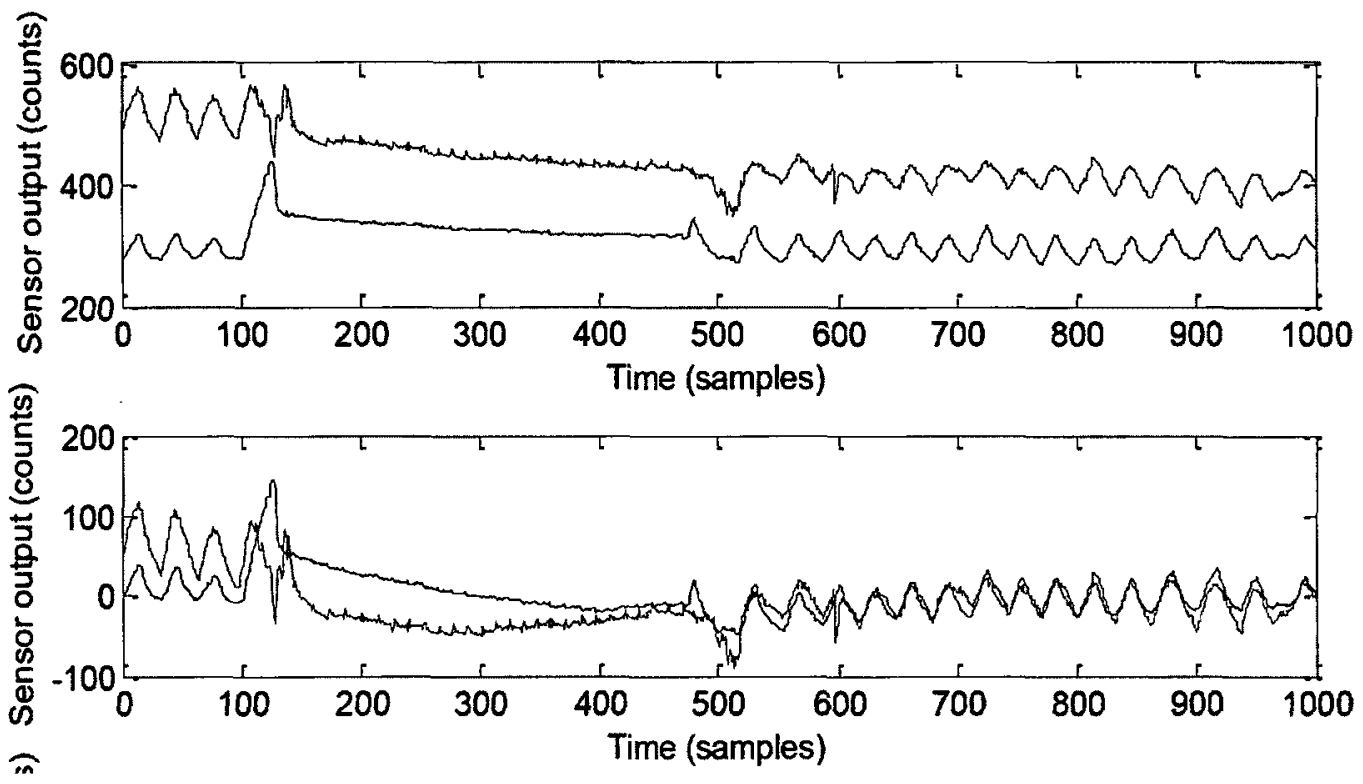

Figure 4.2 Output of two individual pressure sensors for raw data (top), data without mean load (bottom).

The mean load level is due to the weight of the participant loading the sensor and can be removed to recover the breathing signals without weight loading changes. Signal variance was identified to be a main feature for classification of apnea events because it is a measure of the spread, or distribution of the signal over a time interval. A $1 \mathrm{~s}$ moving variance $\left(\sigma^{2}\right)$ see eq. (4.2), was extracted from each of the sensor outputs at each sample time and compared to a threshold value to classify each sample as 'apnea' (if the variance is less than the threshold) and as 'breath' (if the variance is greater than the threshold). 
The variance is calculated by eq. (4.2), where the sensor output for $\mathrm{N}$ consecutive samples is $x(i)=[x(i), x(i+1), \ldots, x(i+N)]$.

$\sigma_{x}(i)=\frac{N}{N-}\left(E\left[x^{2}(i)\right]-E[x(i)]^{2}\right)$

Figure 4.3 shows the $1 \mathrm{~s}$ moving variances for the same two sensor outputs calculated with a rectangular window for the two filtered pressure sensors (top plot). In the figure, the threshold was set to 7 .

The middle plot shows the effect of applying the threshold to the moving variance signal. The bottom plot presents the number of sensors (out of a maximum of 24) who classified
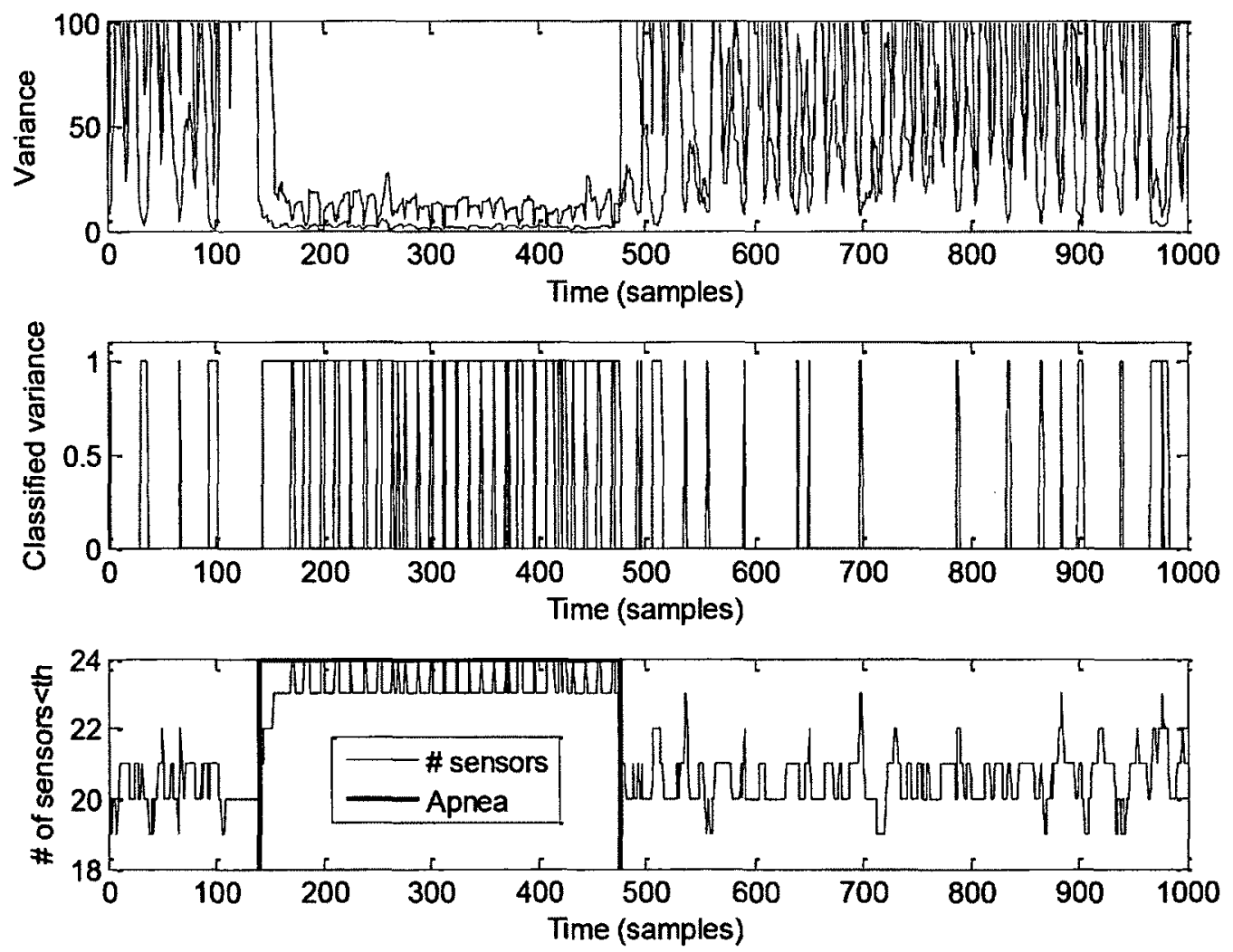

Figure 4.3 Variance for two individual pressure sensors (top), classified moving variance ( $1=$ apnea, $0=$ no apnea) (middle) and number of sensors with variance < threshold (bottom). 
as apnea, where the classification of apnea is assessed every $0.1 \mathrm{~s}$. The apnea recorded by the observer is shown (in red) by the vertical bars in this bottom plot.

Figure 4.4 (top) is a histogram of the $1 \mathrm{~s}$ moving variance signal, for $100 \mathrm{~s}$ of prone breathing for a participant lying on the pressure array. It can be compared to Figure 4.4 (bottom), which is a histogram of the $1 \mathrm{~s}$ moving variance in a $30 \mathrm{~s}$ apnea sample, which has a much smaller range.

For the final step in the block diagram of Figure 4.1, two approaches are considered for sample-based CA detection: the single sensor approach and the multisensor vote
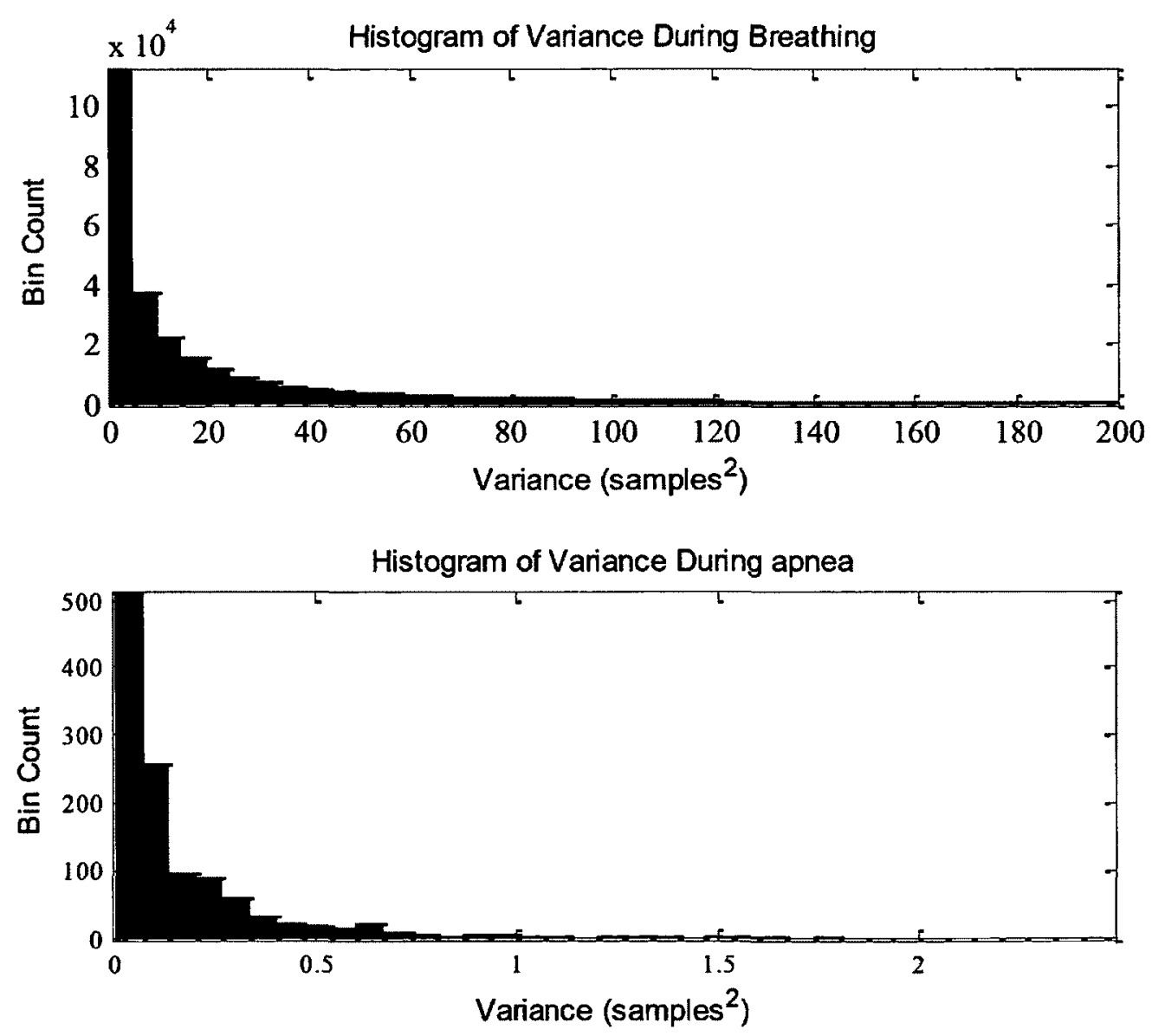

Figure 4.4 Histogram of $1 \mathrm{~s}$ moving variance calculated during prone breathing (top) and prone breath hold (bottom) 
approach. For the single sensor approach, each pressure sensor signal is treated as an experiment, and the results of each of 24 pressure sensor signal per patient file are combined. This is akin to placing one pressure sensor at a random location within the area covered by the mat and studying its classification ability.

In the fused vote method, a vote is made for every sample based on how many sensor outputs were classified as apnea with the number of 'apnea' votes needed to classify a sample as apnea is varied. To address aim ii) Matthew's correlation coefficient (MCC), shown in eq. (4.3), is used to evaluate a classifier with unevenly distributed classes. It is a measure of the quality of a two state classifier, taking into account all four elements of the confusion matrix to measure the performance of the classifier. MCC uses true positives (TP), true negatives (TN), false positives (FP), and false negatives (FN), to calculate a score $[-1,1]$, where +1 represents a perfect prediction, 0 a random prediction and -1 an inverse prediction.

$$
M C C=\frac{T P * T N-\square P * F N}{\sqrt{(T P}+\overline{F P)(T P}+\overline{\square N)(T N}+\overline{T P)(T N}+\overline{\square N)}}
$$

First, the effect of varying the number of required votes for an apnea decision was investigated. Then, two experiments were conducted: the first used the leave one out approach to determine the interparticipant variability in choosing an optimal threshold on the variance. The second experiment divided the data according to participant position and observed the positional effect on classification. The volunteers held their breath for a total of 25 simulated apneas lasting $28.9 \pm 9.4 \mathrm{~s}$. Simulated apneas represented $6 \%$ of all experiment data. To better characterize the classifiers, values for true positive rate (TPR) also known as sensitivity $T P /(T P+F N)$, specificity $T N /(T N+F P)$, and false positive rate 
(1-specificity) are also calculated in addition to the MCC. The threshold value for the variance was tested in 0.05 increments, in a range from 0 to 10 .

\subsection{Results and Verification}

The single sensor method was compared to the multisensor vote method. For the multisensor vote method, the number of votes was varied from 1 to 24 because the sensor array has 24 individual sensors. Figure 4.5 shows the effect of changing the number of votes required for an apnea classification with the false positive rate (FPR) decreasing as the number of votes was increased. The true positive rate (TPR) also decreases, but not as rapidly and stabilized at 21 votes. The MCC has its quickest rise between 21 and 24 votes. This indicates that consensus voting produces the best MCC and the lowest FPR, which are the two aims of the classifier.

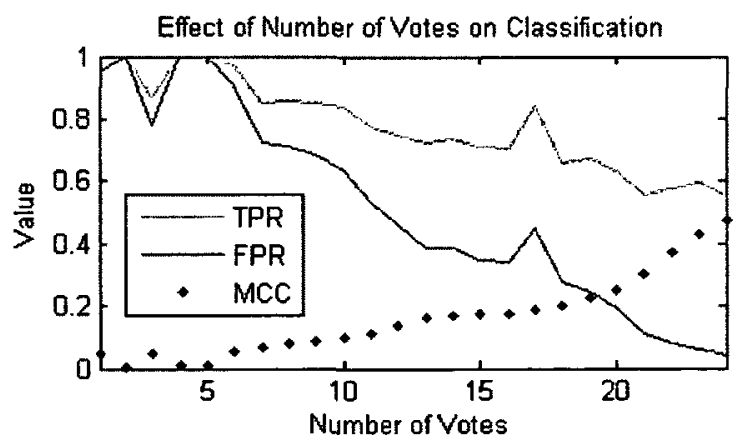

Figure 4.5 Effect of changing the number of votes on classifier performance for $\mathrm{CA}$ detection

Figure 4.6 show the receiver operating characteristic (ROC) curve for CA classification. Curves are shown for $\mathrm{N}=1$ through $\mathrm{N}=24$ votes. As $\mathrm{N}$ increases, the curve progresses from a straight line (chance classifier) to a more squared shape, showing that increasing the number of votes provides superior classification. 


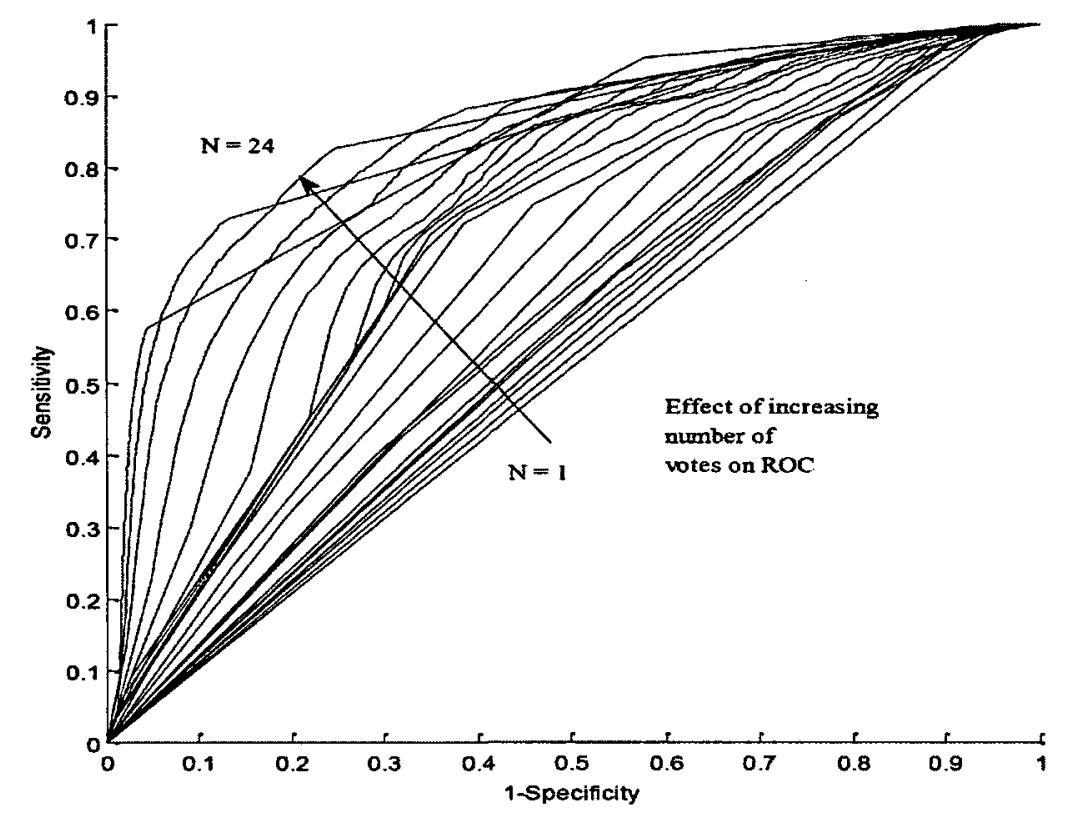

Figure 4.6 Squaring the ROC curve by increasing number of votes

Table 4.1 shows the results from the interparticipant classification using the leave one out approach. The validation results were combined and the average (Avg) value is reported for the classification parameters. The thresholds used for the $1 \mathrm{~s}$ variance that maximized the MCC are similar for each individual participant, indicating that individual participant data had a limited impact on the training.

Table 4.1 Interparticipant $C A$ classification results

\begin{tabular}{|lcccc|l|} 
& Sens & Spe & FPR & MCC & \multicolumn{1}{l|}{ N } \\
& & & & & Votes \\
\hline $\begin{array}{l}\text { Single sensor } \\
\text { Threshold }=1.80\end{array}$ & 0.87 & 0.35 & 0.63 & 0.10 & N/A \\
\hline $\begin{array}{l}\text { Multisensor vote } \\
\text { Threshold }=8.00\end{array}$ & 0.54 & 0.96 & 0.05 & 0.48 & 24 \\
\hline
\end{tabular}

All MCC values are positives, indicating the classification is better than random, but still quite low. Across participants, the sensitivity and specificity values were consistent 
(standard deviation $\leq 0.1$ ), suggesting that the feature of pressure variance is common across all participants as an indicator of simulated apneas.

Table 4.2 presents the effect of lying position on the classification for the complimentary methods. The threshold with the best MCC value is lowest for the side position in both approaches. The higher threshold and lower sensitivity in the multisensor vote method are in part due to the ballistocardiogram signal. This pulse-like signal appears in many sensors, during many simulated apneas, and contributes to a higher variance. The single sensor method has a high sensitivity; however the FPR consistently exceeds 0.5 , which is unacceptable for clinical applications. The FPR from the multisensor method is acceptable for many clinical applications, in all positions tested.

Table 4.2 CA classification results separated by position

\begin{tabular}{|c|c|c|c|c|c|c|c|}
\hline & ipnea & $\begin{array}{l}\text { Thresh- } \\
\text { old }\end{array}$ & Sens & Spe & FPR & $\mathrm{MCC}$ & \#Votes \\
\hline \multicolumn{8}{|c|}{ Single Sensor } \\
\hline Prone & 7.34 & 7 & 0.86 & 0.41 & 0.59 & 0.15 & N/A \\
\hline Supine & 5.55 & 1.65 & 0.80 & 0.42 & 0.58 & 0.11 & $\mathrm{~N} / \mathrm{A}$ \\
\hline Side & 6.18 & 0.15 & 0.83 & 0.43 & 0.57 & 0.12 & N/A \\
\hline \multicolumn{8}{|c|}{ Multisensor Vote } \\
\hline Prone & 7.34 & 10 & 0.52 & 0.97 & 0.03 & 0.53 & 23 \\
\hline Supine & 5.55 & 7 & 0.69 & 0.97 & 0.03 & 0.63 & 24 \\
\hline Side & 6.18 & 4.8 & 0.71 & 0.95 & 0.05 & 0.57 & 24 \\
\hline
\end{tabular}

The multisensor vote approach shows how optimizing the number of sensor votes decrease FPs and increases the MCC. The distribution of the pressure sensor output's variance changes during breathing events and this allows the $1 \mathrm{~s}$ variance to be a useful classification feature. The experiment revealed that in side lying more individual sensors were unloaded or saturated. These sensors had a very small variance and were very often classified as apnea regardless of participant activity. As well, cardiogenic oscillations were observed during simulated apneas which resulted in areas of higher variance. 


\subsection{Single Participant Multiple Mattress Trial - Controlled Experiment B}

Controlled experiment B address some challenges with controlled experiment A which were a) a high number of false detections and b) reports that some participants found the sensor uncomfortable when side lying ${ }^{5}$. For this experiment, a single subject provided data and the pressure sensor array was placed beneath the mattress as intended in clinical use as shown in the diagram of Figure 4.7.

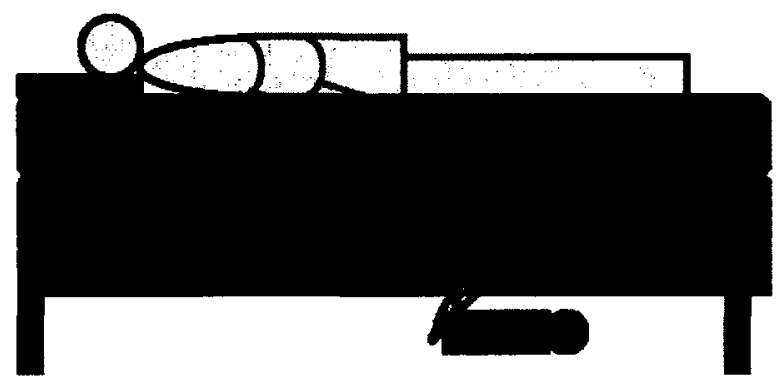

Figure 4.7 Model of experimental setup for controlled experiment with single sensor placed beneath mattress

In the previous section, the single sensor method has high sensitivity, while the multisensor method has high specificity. Presently, two methods are investigated for their ability to increase the accuracy of apnea detections: i) moving average windowing (MA) and ii) window elimination (WE). In this experiment, the effect of windowing on samplebased classification was investigated. The estimated apnea durations were compared to measurements from two commercial respibands bands which were placed around the chest and the abdomen of the participant to measure the change in circumference created by airflow into the lungs. The bands are sampled at $12 \mathrm{~Hz}$, with 12-bit resolution and also time stamped to a single log file.

\footnotetext{
${ }^{5}$ A significant portion of Sections 3.4 and 3.5 is published in [3].
} 
A subject followed a protocol of breathing exercises and movements which included normal breathing, shallow breathing, deep breathing, and relaxed breath-holding to simulate apneas while lying prone, supine, and on each side. This sequence was repeated on nine mattresses. Body positioning and the onset and offset of apneas were logged and time stamped. The respiband and pressure sensor samples were time-aligned using the time stamps present in both files. Manual analysis of the time stamped log notes and the respiband data yielded two single output files: apnea and body position. The simulated CAs in the dataset are described in Table 4.3. Simulated CAs represent $4 \%$ of the collected data. Participant data was separated into a training set ( $2 / 3$ of data) and a test set (1/3 of data). Figure 4.8 shows the block diagram for the windowing method.

Table 4.3 Properties of recorded apneas in dataset

\begin{tabular}{|lllllll|} 
& \multicolumn{2}{l}{ Complete Data } & \multicolumn{2}{c|}{ Training Set } & \multicolumn{2}{l|}{ Test Set } \\
& $\#$ & Length (s) & $\#$ & Length (s) & $\#$ & Length (s) \\
\hline Prone & 12 & $12.7 \pm 3.7$ & 7 & $14.2 \pm 3.0$ & 5 & $10.6 \pm 3.8$ \\
\hline Supine & 8 & $17.2 \pm 5.0$ & 5 & $16.1 \pm 2.9$ & 3 & $19.0 \pm 7.9$ \\
\hline Side & 9 & $15.0 \pm 2.4$ & 6 & $14.6 \pm 2.8$ & 3 & $16.0 \pm 1.7$ \\
\hline Total & 29 & & 18 & & 11 & \\
\hline
\end{tabular}

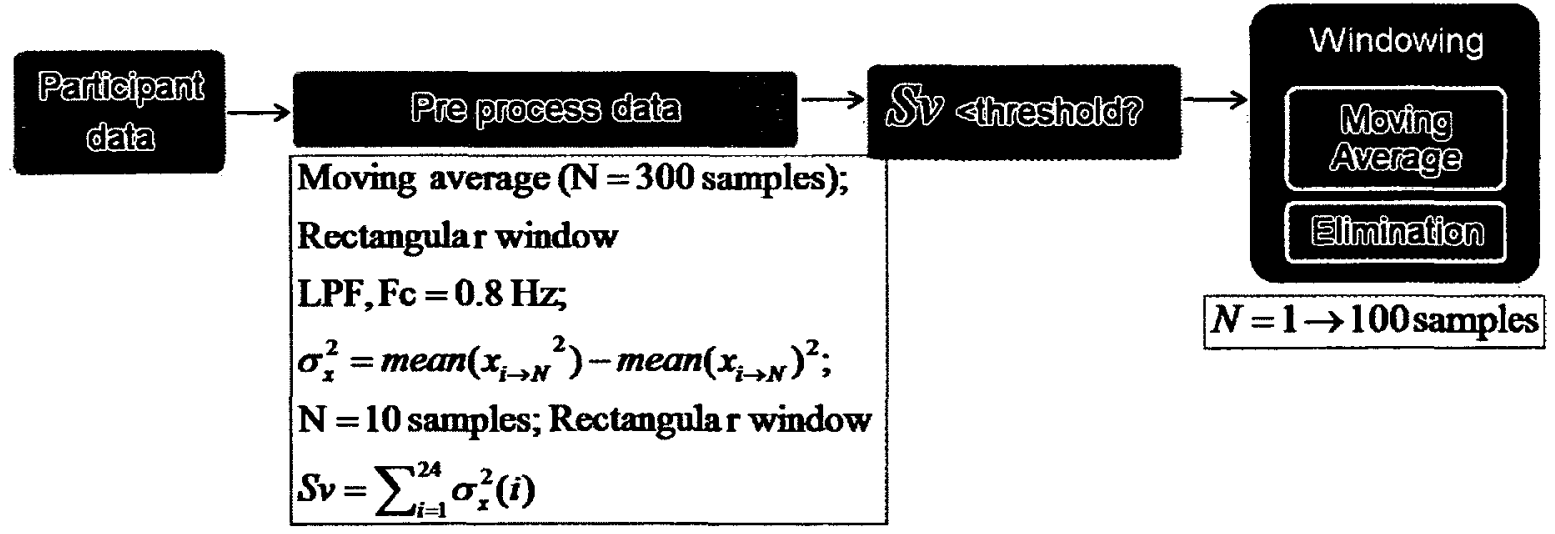

Figure 4.8 Block diagram for windowing approach showing pre-processing, classification and windowing steps. 
Figure 4.9 shows the output of two selected pressure sensors during the data preprocessing stage, as shown in Figure 4.2. The figure shows the data output from the acquisition box in the top plot. The middle plot shows the raw data with the mean loading value removed. The signals are now centered around zero. This time, the bottom plot shows the two pressure sensor outputs after removal of the mean loading value and after filtering with a low pass filter and time-shifting the signal to compensate for the group delay of the filter.Initial classification of the apnea state was performed by calculating a moving $1 \mathrm{~s}$ variance and classifying each sample as 'apnea' or 'no apnea' according to thresholds defined in Section 4.3 [2]. These thresholds vary according to body position.

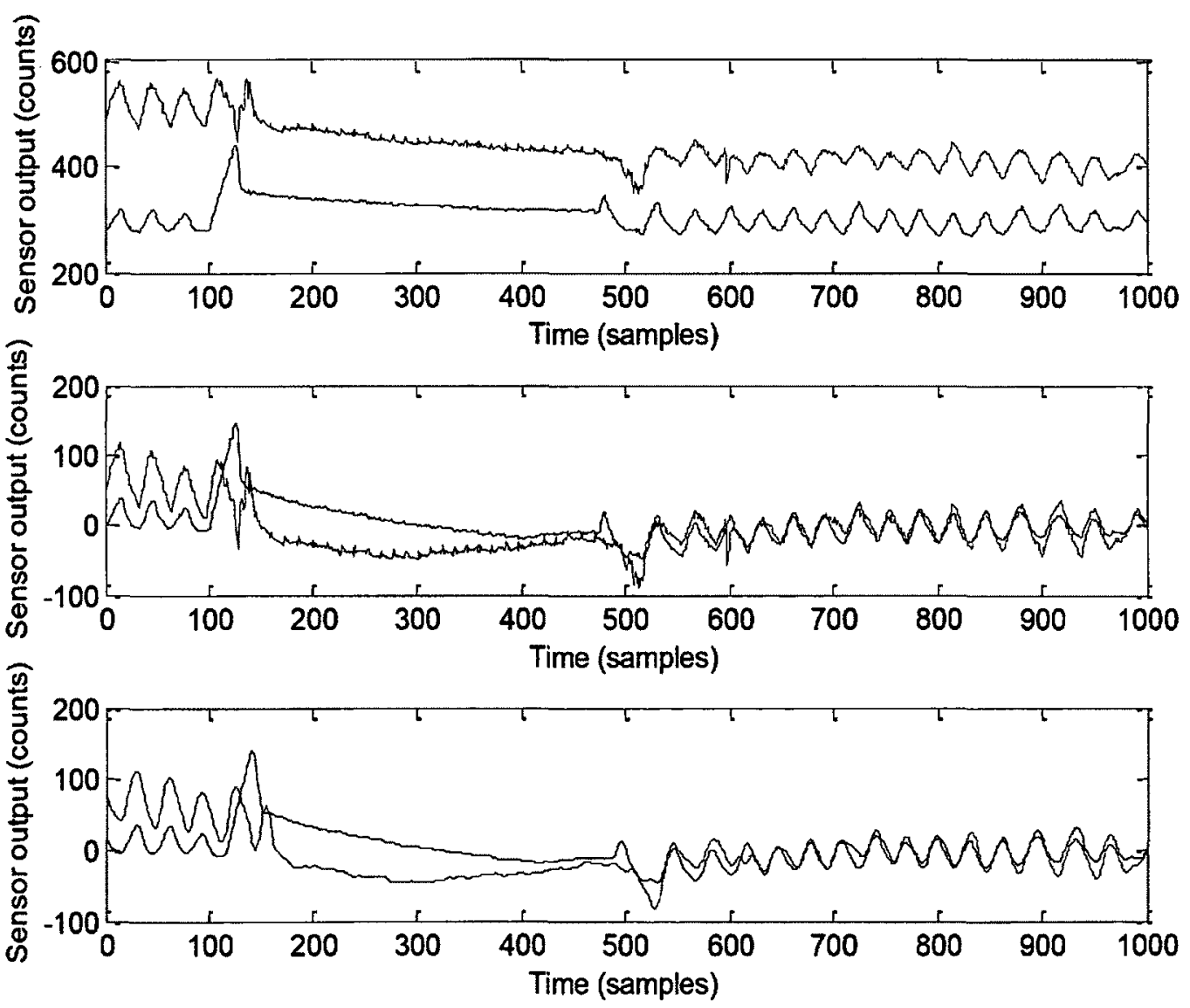

Figure 4.9 Output of two individual pressure sensors for raw data (top), data without mean load level (middle) and after filtering with $\mathrm{fc}=0.8 \mathrm{~Hz}$ (bottom). 
The MA and WE methods were applied to the initial classification results to reduce short, falsely classified segments. A MA filter with a window size ranging between 1 and 100 samples was applied to the classified signal. The signal was then rounded to the nearest integer $(1=$ apnea, $0=$ no apnea).Prior to comparison with the apnea annotations, the rounded signal was shifted forward by a number of samples equal to half the window size to compensate for the filter delay. Previous analysis has shown that detected apneas are most often classified as long positive segments whereas FP areas are often comprised of many short segments. Therefore the WE method uses a windowing approach to remove positive segments lasting from 1 to 100 samples.

Relevant signals for this CA detection method are shown in Figure 4.10. The top plot presents the sum of the variance signals from each of the 24 pressure sensors in the array for $60 \mathrm{~s}$ of data. The middle plot shows the classified signal and location of the apnea.
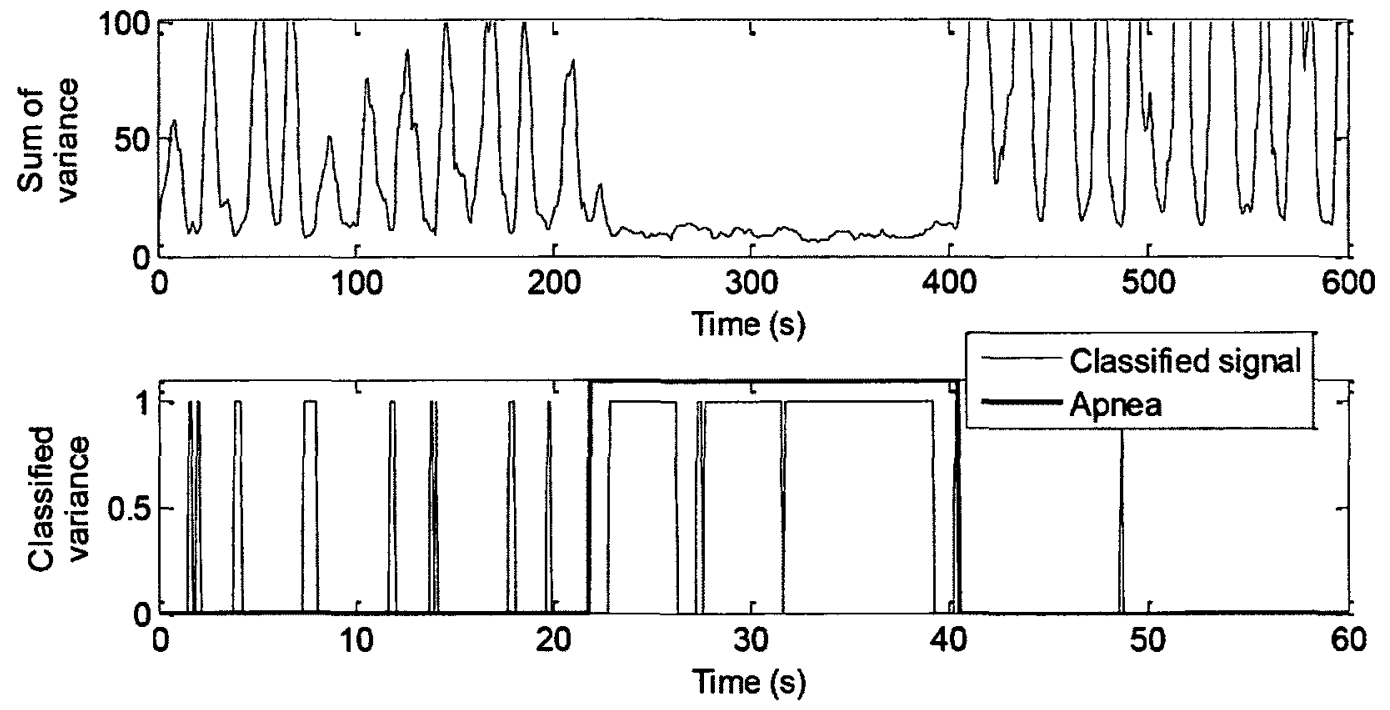

Figure 4.10 Sum of the variance from all 24 pressure sensors in the array (top), classified moving variance $(1=$ apnea, $0=$ no apnea) (bottom) 
Figure 4.11 shows the respiband signals (top 2 plots) and output of the pressure sensor array (bottom plot) for a $70 \mathrm{~s}$ period which includes a simulated CA (samples 3200 3400). The figure also shows that the start and end of apneas are sharper in the pressure data due to the responsiveness of the pressure array.
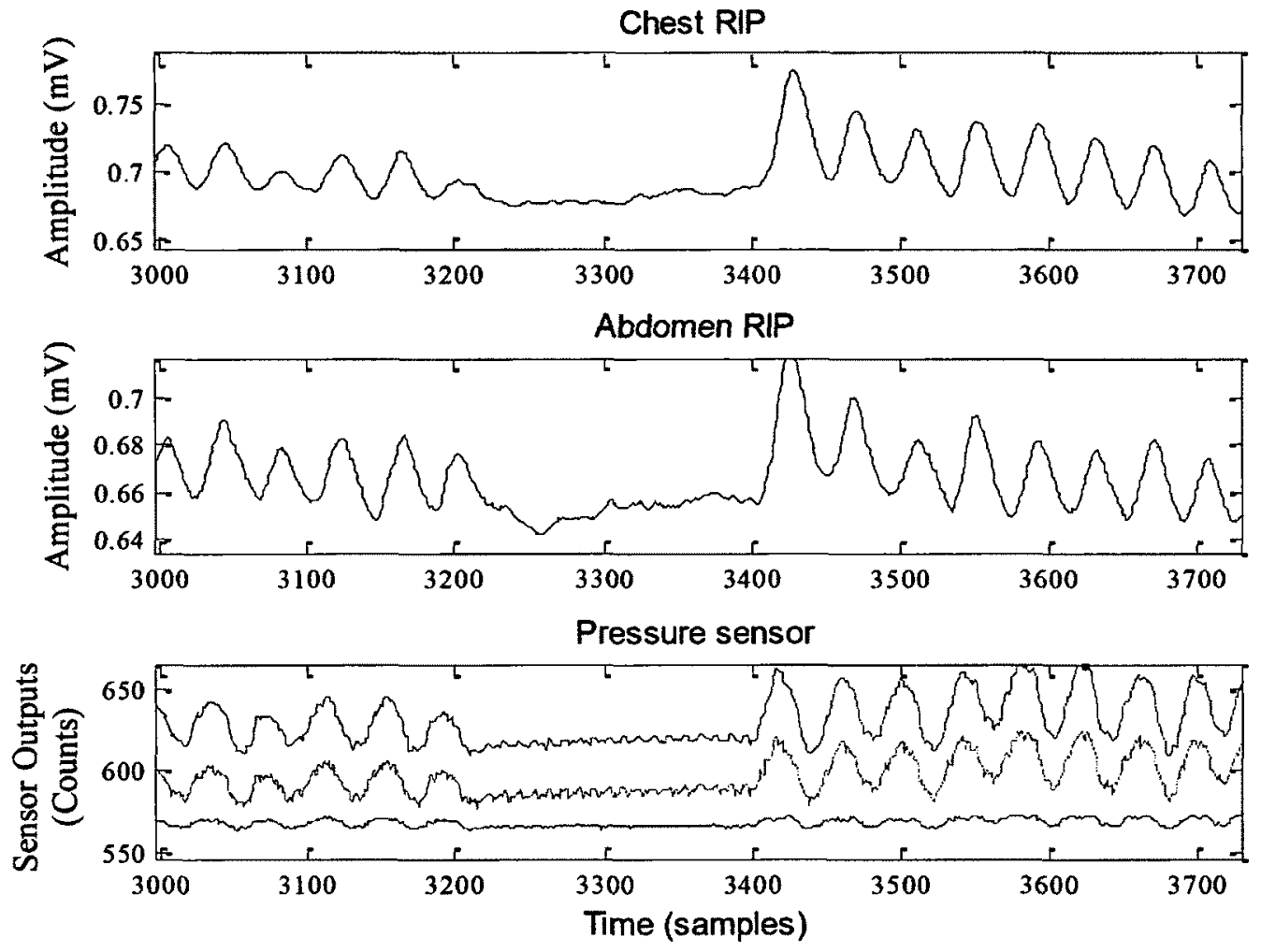

Figure 4.11 Chest and abdominal respiband signals and selected pressure sensor outputs during $\mathrm{CA}$ in controlled experiment

Figure 4.12 shows an example of the initial classification applied to one apnea. The apnea occurs between samples 8120 and 8320 . The classification resulted in many short FP segments (samples 8443-9110 and 9431-10000). The longest TP segment corresponds to the actual apnea (samples 8126-8310). Two long periods of TN occur during deep 
breathing (samples 8732-8874), and during a small muscular movement (samples 92629430).

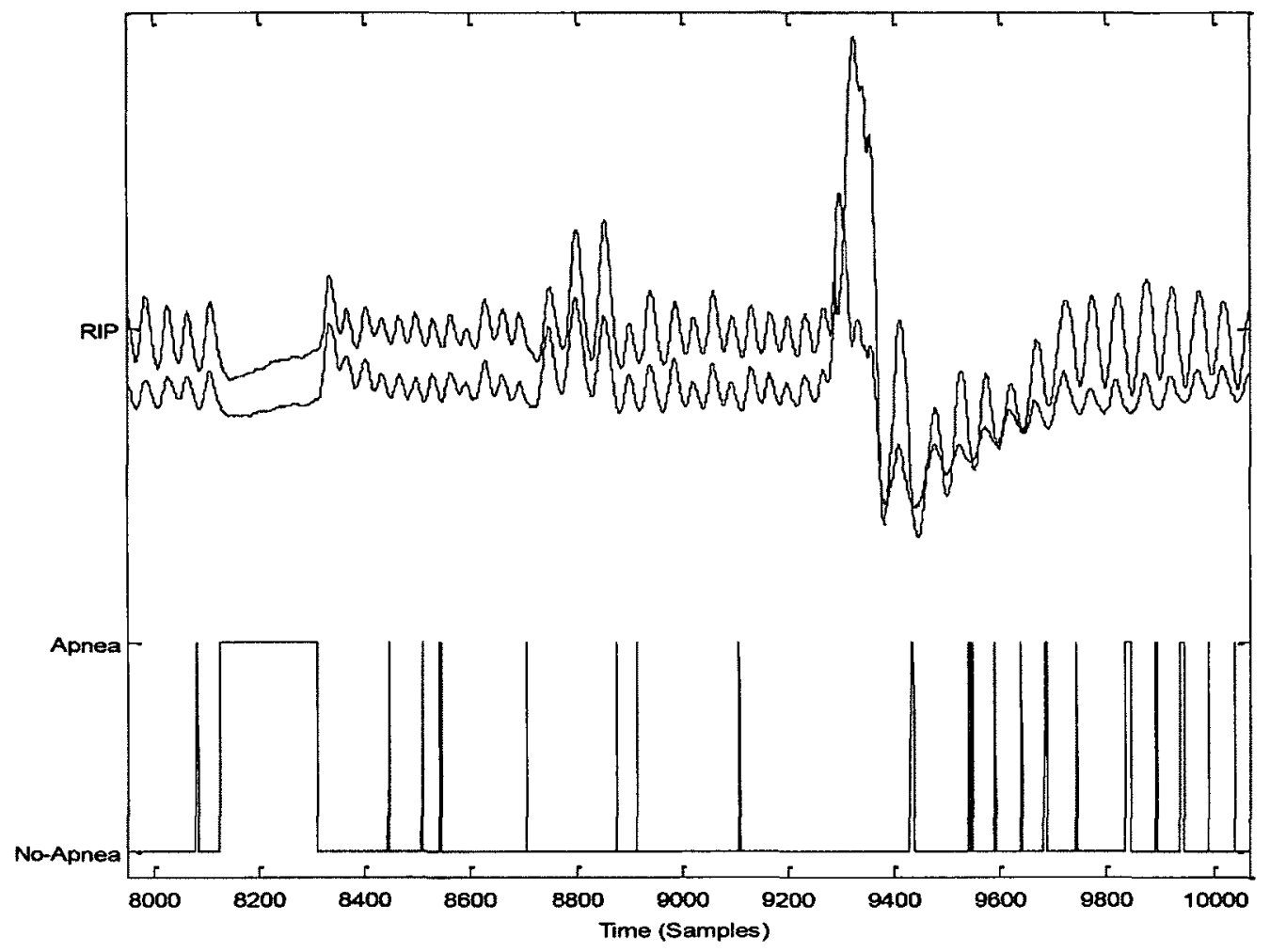

Figure 4.12 Respibands and classified moving variance during breathing and CA

The effect of each windowing method applied the classified moving variance is shown in the following figure. The top plot of Figure 4.13 shows how the MA method merged smaller sections of positive segments to create longer segments, and also removed some small positive segments. The bottom plot shows the elimination of positive segments in the WE method, both during regular breathing and during the apnea. 

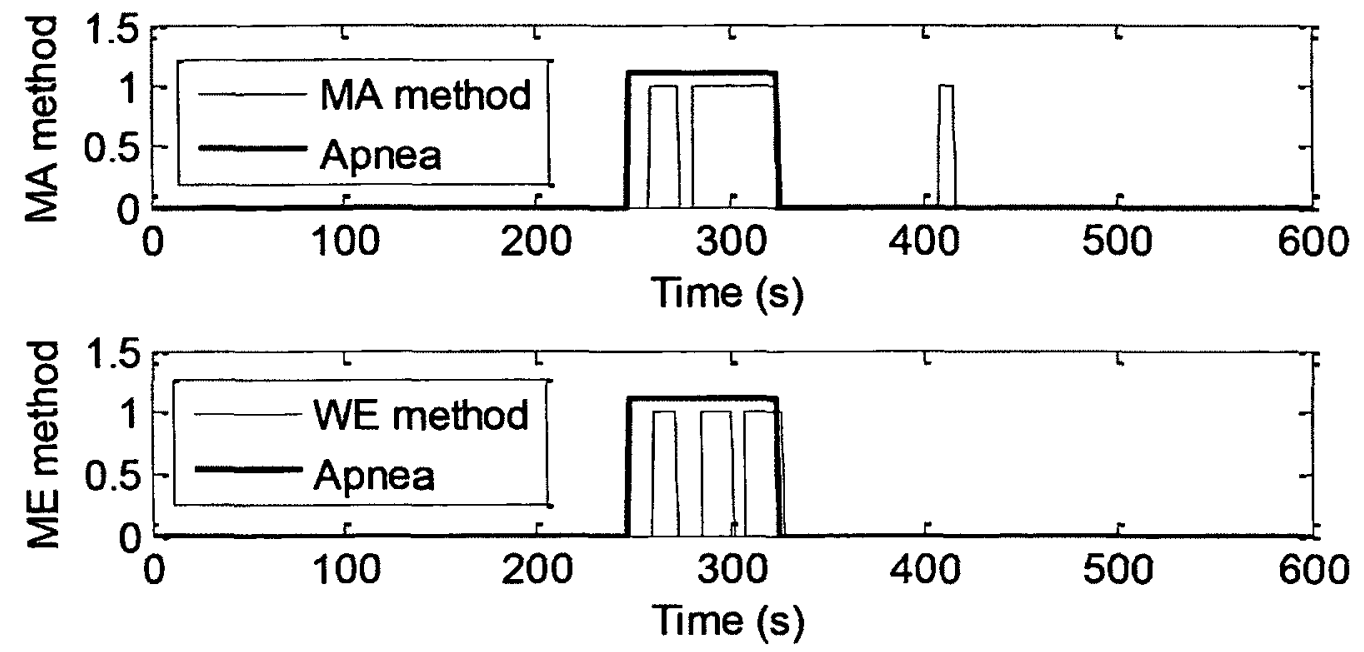

Figure 4.13 Windowing methods applied to classified moving variance signal

\subsection{Results and Verification}

Results for the test set are shown in Table 4.4. The results for MA and WE method are shown for their optimum window lengths, chosen by maximizing the MCC over the training data.

Table 4.4 Simulated CA classification results for test set

\begin{tabular}{|c|c|c|c|}
\hline ( & Initial & MA & WE \\
\hline Sens & 0.87 & 0.82 & 0.75 \\
\hline Spe & 0.35 & 0.69 & 0.92 \\
\hline FPR & 0.63 & 0.31 & 0.08 \\
\hline MCC & 0.10 & 0.30 & 0.45 \\
\hline Optimal window size & & 30 & 68 \\
\hline
\end{tabular}

The classification accuracy was affected by the window size as shown in Figure 4.14, while the classification of the WE method is shown in Figure 4.15. 

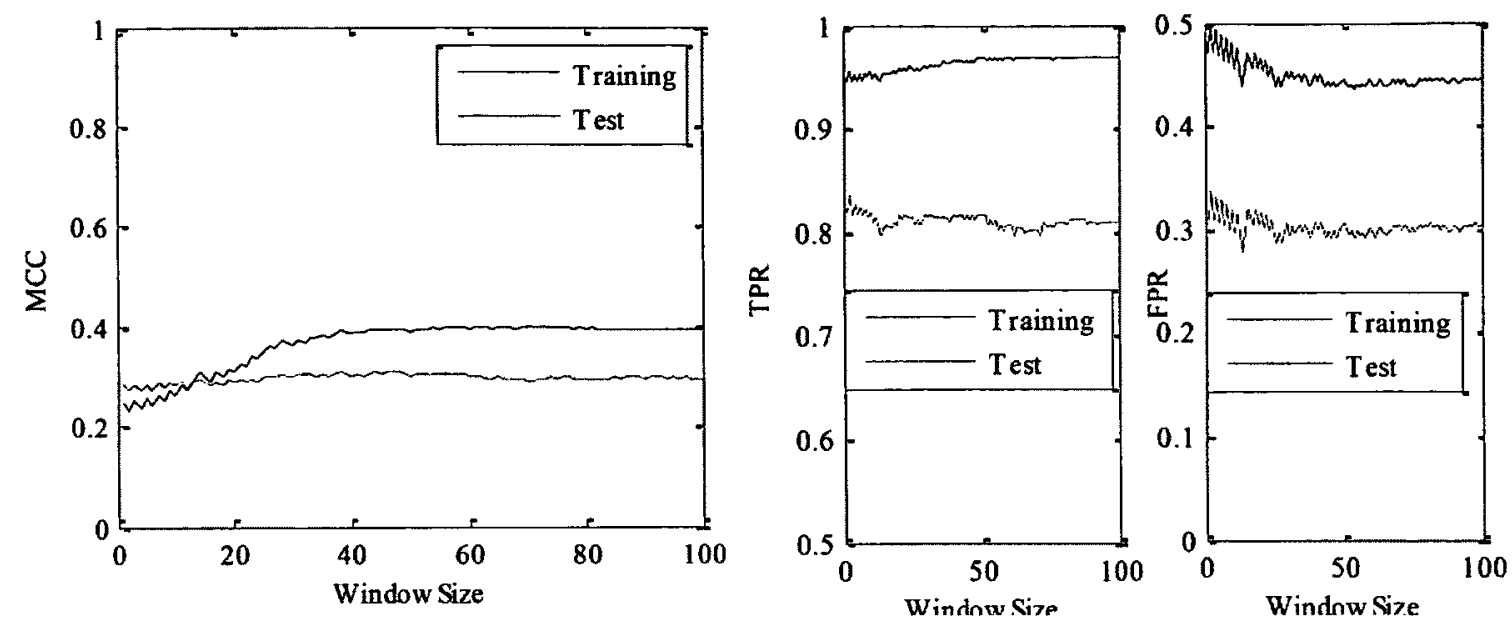

Figure 4.14 Effect of window size on MCC (left) and TPR and FPR (right) on CA detection for the MA classifier
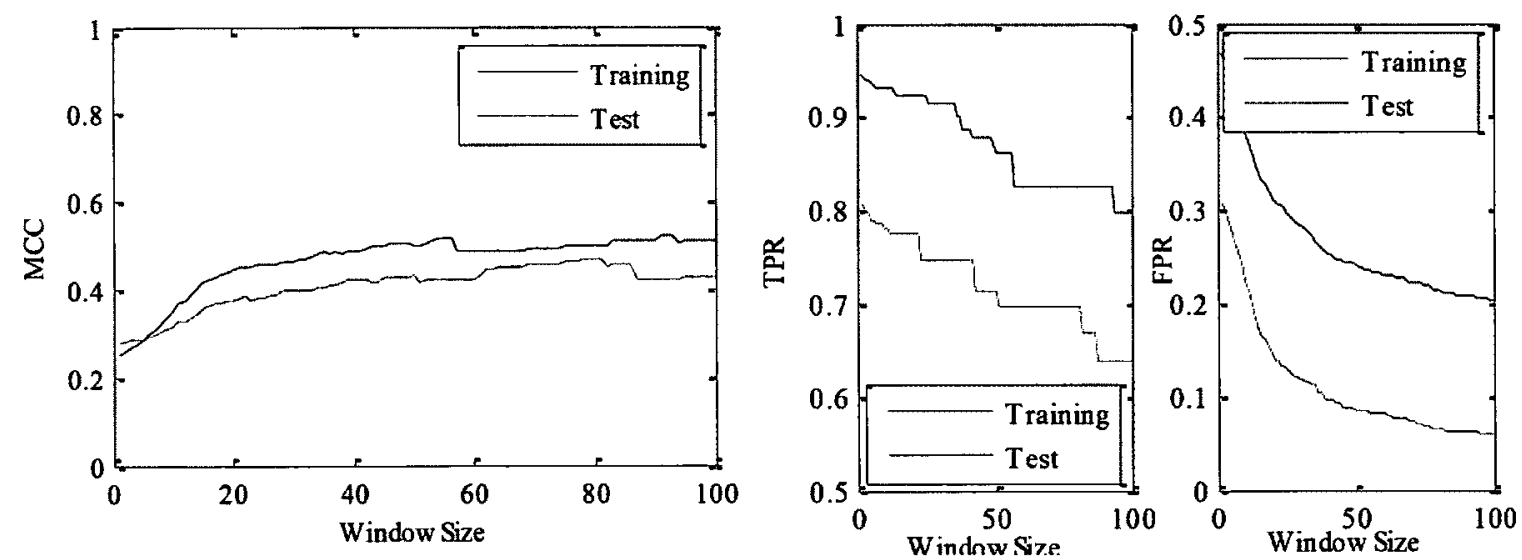

Figure 4.15 Effect of window size on MCC (left) and TPR and FPR (right) on CA detection for the WE classifier

The MA increased classifier performance up to a window size of 30 , but failed to reduce the FPR. The WE method significantly increased the MCC ( $\geq 14 \%)$ and specificity with a slight decrease in sensitivity. The error on apnea duration estimates for both methods are shown in Table 4.5. Most apneas were estimated within $1 \mathrm{~s}$ of their actual duration. 
Table 4.5 Error on apnea duration estimates for both windowing methods

\begin{tabular}{|llllllll|} 
& $\pm 1 s$ & $\pm 2 s$ & $\pm 3 s$ & $\pm 5 s$ & $\pm 10 s$ & $>10 s$ & Missed \\
\hline MA & 18 & 7 & 1 & 0 & 1 & 1 & 1 \\
\hline WE & 18 & 1 & 0 & 1 & 1 & 0 & 5 \\
\hline
\end{tabular}

Durations were calculated according to the diagrams of Figure 4.16. The duration of apneas in the MA classifier were measured from onset to offset of the segment as seen in Figure 4.16 (top). For the WE classifier, gaps between classified apnea segments were included in the apnea duration if $|g a p| \leq W_{W E}$, as shown in Figure 4.16 (bottom). In this case, only two segments remain after the WE algorithm was applied. Most apneas were detected within one second of their actual duration, due in part to the sharp boundaries between breathing and apneas provided by the pressure sensor array.
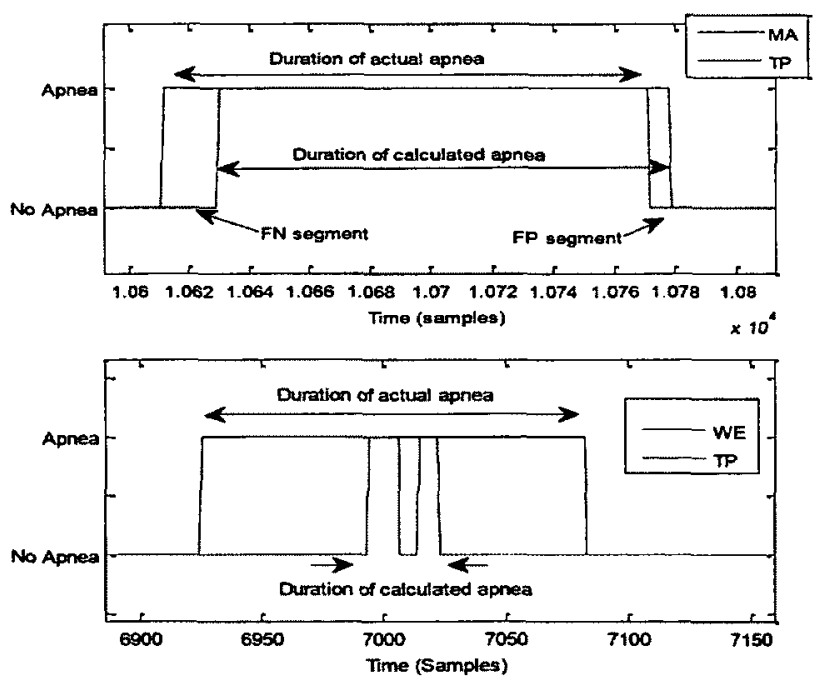

Figure 4.16 Calculating the apnea duration for the MA (top) and the WE (bottom) classifier 
The WE method compares favorably to the MA method, with an average error of $0.7 \pm 1.2$ $\mathrm{s}$, compared to $0.8 \pm 1.7 \mathrm{~s}$ for MA windowing. Apnea duration was not correlated with the estimation error as has been reported in the literature with human scorers [41].

The timing difference between the onset of the apnea in the pressure sensor and respibands was $1.2 \pm 2.8 \mathrm{~s}$ at the start and $1.2 \pm 0.7 \mathrm{~s}$ at the end, with the respibands lagging the pressure sensor in both cases as shown in Figure 4.17. The end of apneas, especially longer ones, is generally sharper in the initial stage as the person 'gasps' for air. The difference in calculated apnea duration however was small; the apneas measured with the pressure sensor were longer by an average of $0.1 \pm 0.3 \mathrm{~s}$.

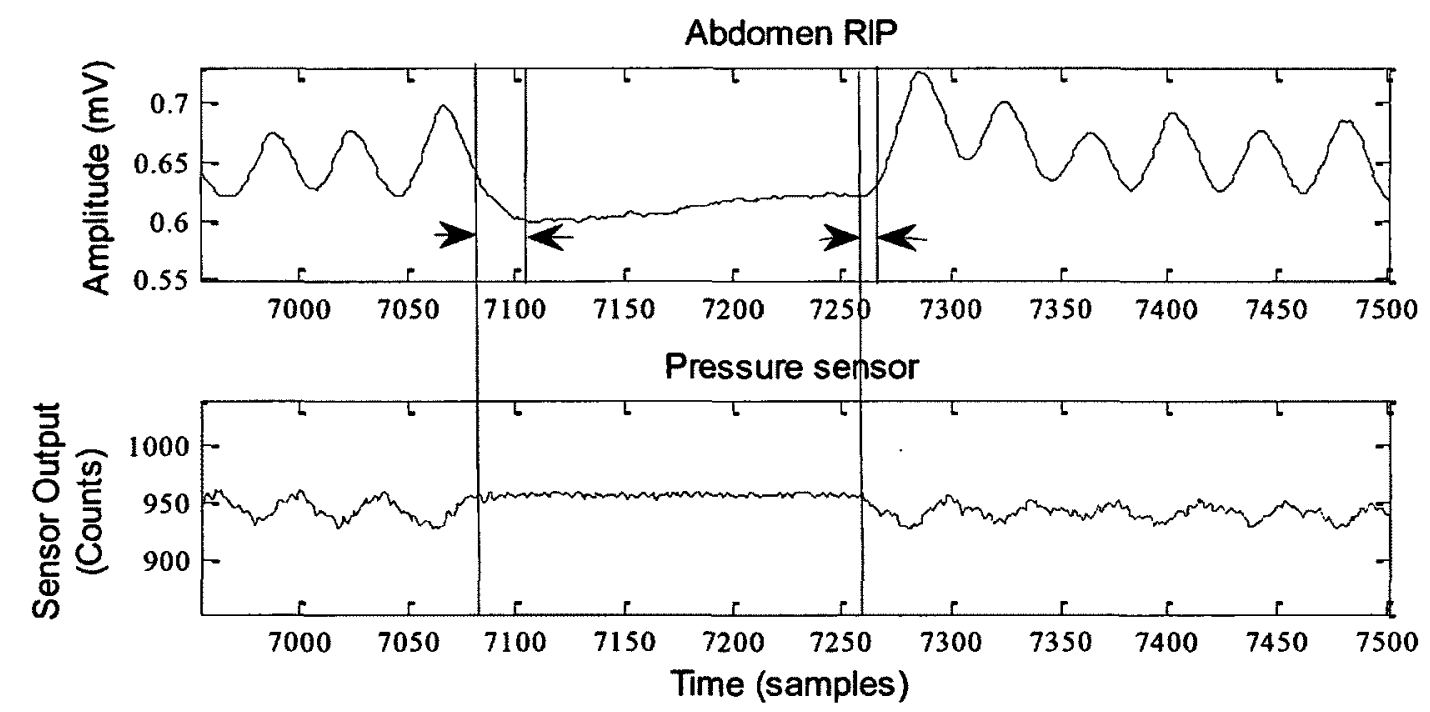

\section{Figure 4.17 Comparison of apnea onset and offset for the abdomen respiband and one pressure sensor output}

The initial sample-based classification was able to detect apneas, but resulted in many spurious FPs and some missed apneas. By connecting segments together, the MA method was able to increase the sensitivity at the expense of a slight FPR increase. The WE 
method, by instead removing short segments of positive results, improved the FPR, with only a modest decrease in the TPR. However, a number of apnea segments that were initially classified as a sequence of short duration apneas were no longer detected.

A decrease in the number of transitions between the two states in the classified signal was observed with both methods. The number of transitions in the MA method is 520 and in the WE method is 166 , a reduction of $89 \%$ and $96 \%$ respectively. The placement of the sensor underneath the mattress did not present any discomfort to the volunteers.

\subsection{Event-Based Detection - Controlled Experiment C}

Close examination of controlled experiment B's data revealed that some mattresses reacted differently to simulated CAs. As such, this section presents the use of an eventbased model of CAs and uses a relative rather than hard-coded threshold on the moving variance, allowing the algorithm to adapt to the different signal strengths seen with different mattress types and individual body weights ${ }^{6}$. This section presents a parametric apnea model to allow event-based detection and classification based on modifications of Han \& al.'s work [76]. The model is modified to include a new parameter $\left(t_{2}\right)$ and to use a relative threshold $d$ instead of a fixed value. The same dataset as controlled experiment B was used for the parametric model [3], [2]. One foam, one coil and one futon mattress were used to train the algorithm and select the model parameters; these are indicated by an asterix beside the mattress type. The remaining mattresses ( 3 coil, 3 foam including institutional) were used for the test set. After the experiment completion, two additional pressure-respiband signal pairs were collected from a secondary participant whose weight

\footnotetext{
${ }^{6}$ A significant portion of Section 4.5 is published in [4].
} 
was $16 \mathrm{~kg}$ more than the first participant. These form the validation set and are indicated by a superscript $\mathrm{V}$ in Table 4.6 .

Table 4.6 Position and mattress type for each CA during controlled experiment

\begin{tabular}{|llllll|}
$\begin{array}{l}\text { Mattress } \\
\text { Type }\end{array}$ & $\begin{array}{l}\text { Mattress } \\
\text { Thickness }(\mathrm{cm})\end{array}$ & $\begin{array}{l}\text { Total\# } \\
\text { apneas }\end{array}$ & $\begin{array}{l}\text { \#Supine } \\
\text { apneas }\end{array}$ & $\begin{array}{l}\text { \#Side } \\
\text { apnea }\end{array}$ & $\begin{array}{l}\text { \#Prone } \\
\text { apneas }\end{array}$ \\
\hline${ }^{*}$ Coil & 35 & 3 & 1 & 1 & 1 \\
\hline Coil & 33 & 4 & 1 & 1 & 2 \\
\hline Coil & 23 & 3 & 1 & 1 & 1 \\
\hline Coil & 18 & 3 & 1 & 1 & 1 \\
\hline Foam & 13 & 4 & 1 & 1 & 2 \\
\hline Foam & 15.2 & 4 & 1 & 1 & 2 \\
\hline *Foam & 15.2 & 3 & 1 & 1 & 1 \\
\hline Foam & 15.2 & 3 & 1 & 1 & 1 \\
\hline 'Foam & 20.2 & 3 & 1 & 1 & 1 \\
\hline *Futon & 15.2 & 6 & 1 & 1 & 4 \\
\hline Futon & 15.2 & 4 & 1 & 0 & 2 \\
\hline
\end{tabular}

training set, ${ }^{\nabla}$ validation set, ${ }^{\mathrm{L}}$ latex mattress

\subsubsection{Detection Approach}

The proposed approach searches the moving variance signal for a sudden decrease located between two areas of higher variance. Upon finding a decrease it then calculates the length of the decrease. The apnea model shown in Figure 4.18 is comprised of five parameters. The model's parameters are:

$\mathrm{N}$ : number of samples in the moving variance

$t_{1}$ : time for moving variance to decrease by $d \%$ to flag apnea onset

$\mathrm{d}: \%$ decrease from baseline/current moving variance required to start counting $\mathrm{t}_{3}$ samples as measured by the percentage difference between $d_{t}$ and $d_{b}$, the value of the variance at the top and bottom of an event as shown in Figure 4.19.

$t_{2}$ : time allotted for moving variance to return to baseline in order to flag apnea offset 
$t_{3}$ : portion of apnea where moving variance $\leq \% d$ decrease, $t_{3}$ values smaller than 10 s are discarded in this experiment as they do not meet the clinical criteria for CAs discussed earlier.

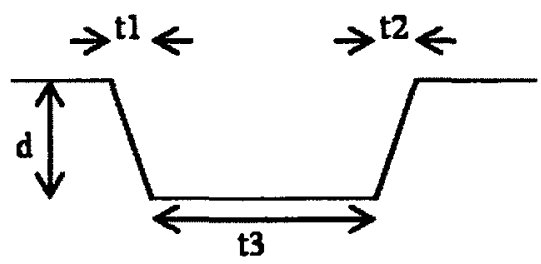

Figure 4.18 Four parameter apnea model used to detect CAs in controlled experiment data

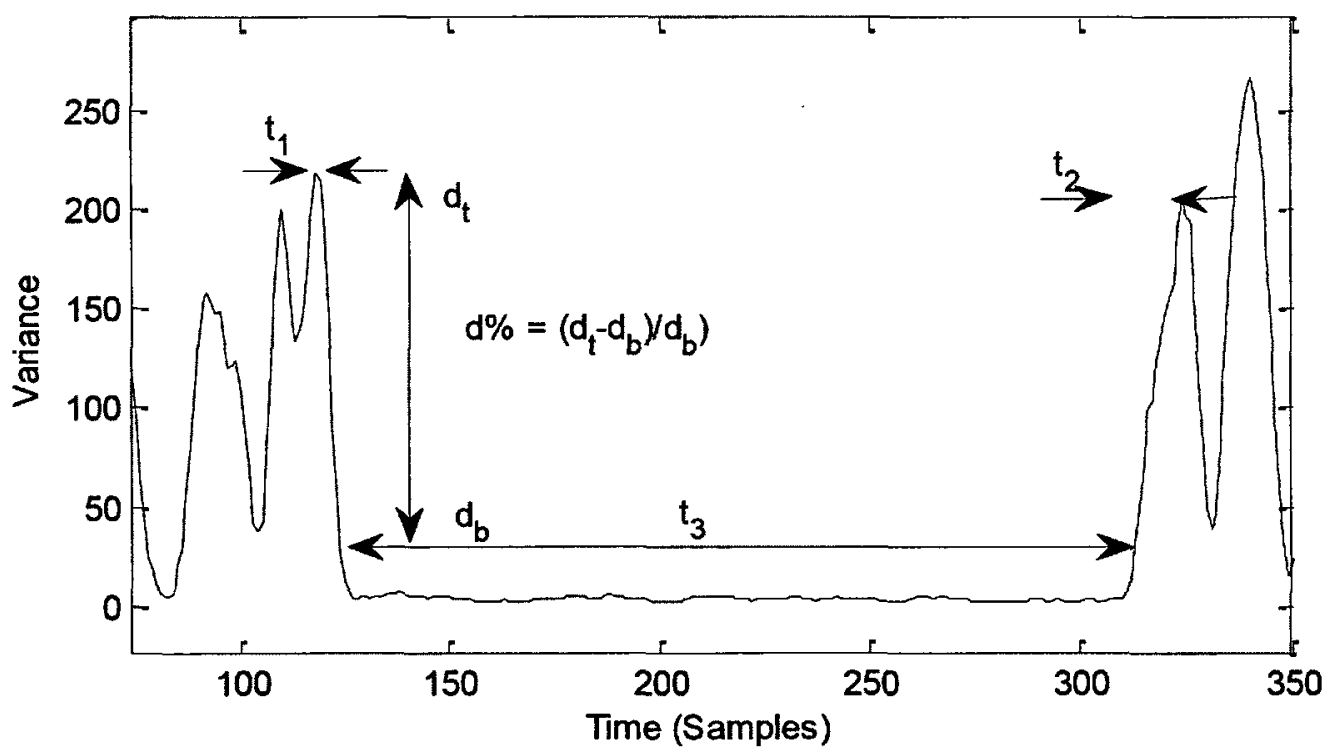

Figure 4.19 Four parameter apnea model applied to an apnea

Figure 4.20 shows the block diagram for the parametric apnea model presented in this section. The figure shows the preprocessing steps executed before the model is applied. 


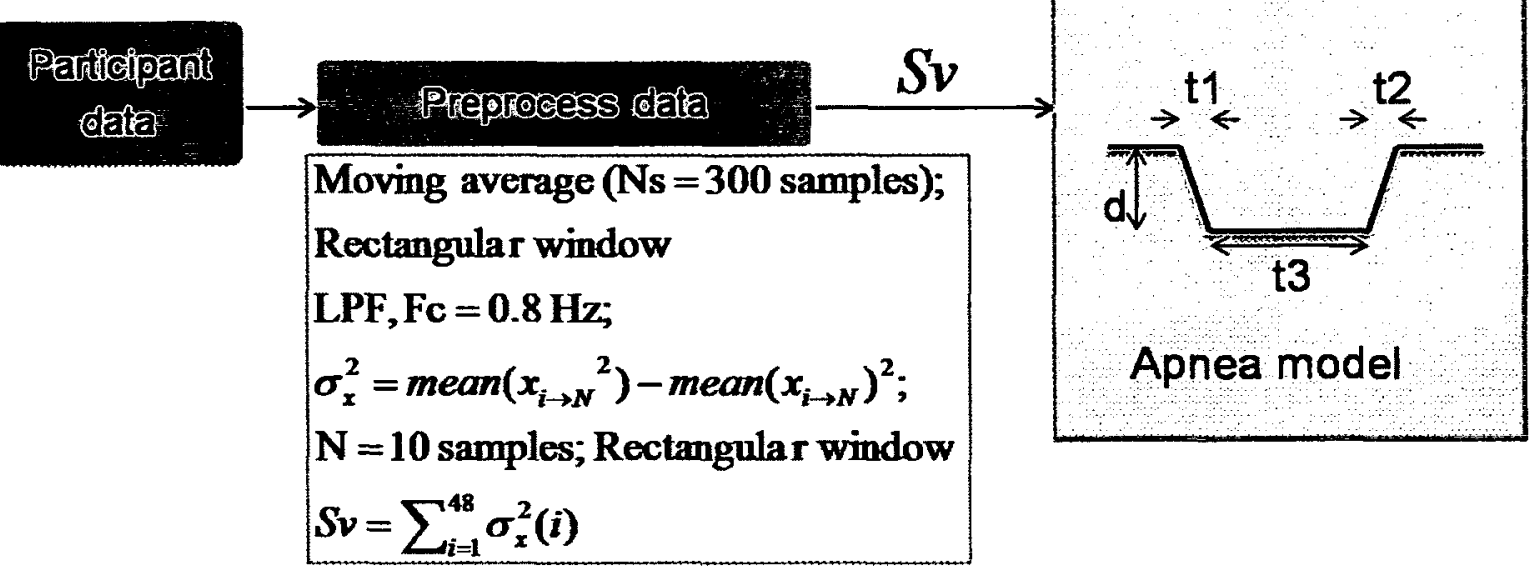

Figure 4.20 Parametric apnea model block diagram

Figure 4.21 presents the framework that was applied to the training set mattresses to find the optimal moving variance length and model parameters. The parameter values were selected, and the model then applied to the remaining $2 / 3$ of data which comprise the test set, and then later to the validation set. This framework applies the model to the moving variance signal using all possible permutations of parameters $d, t_{1}, t_{2}$ and $N$. To select parameters, the classification results were grouped by $d, t_{l}, t_{2}$ and $N$ and then plotted against the MCC and ROC curve to observe each parameter's effect on the results. In the final parameter selection, each parameter was tuned to maximize the MCC.

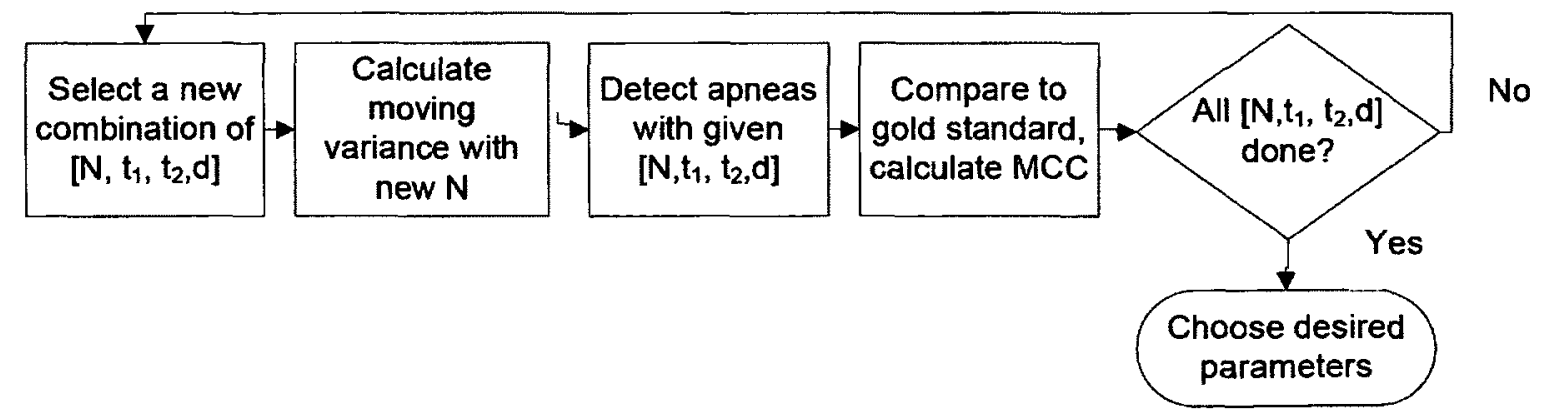

Figure 4.21 Parameter selection framework 
In controlled experiments A (multisensor vote method) all sensors had to agree on low variance value and one sensor could veto the apnea decision. In this case, sensors without breathing information will not show the relative drop in the variance. A new approach is presented here. First, the moving variance $\left(\sigma^{2}\right)$ from each sensor output $(x)$ for each sample time using a window of $N$ samples is calculated as in eq. (4.4). Then all moving variance signals are summed (Smvar) together as in eq. (4.5).

$$
\begin{aligned}
& \sigma_{\mathrm{s}}(\mathrm{i})=\operatorname{mvar}(\mathrm{x}(\mathrm{i}) \ldots \mathrm{x}(\mathrm{i}+\mathrm{N}-1)) \\
& S m \text { var }=\sum_{i=1}^{24} \sigma_{x}(i)
\end{aligned}
$$

\subsubsection{Context-Aware Error Correction}

Certain scenarios were identified as likely to cause FPs. As such, possible apnea segments occurring within the following situations were reverted to non-apnea status:

1. Apparent apneas lasting more than $60 \mathrm{~s}$ : these were removed during processing.

2. Movement artifacts: After movements or position changes, variances decrease substantially and could be misconstrued as apneas. By not allowing apneas to be detected for a duration equal to the window length plus 50 samples after any position change or muscular movement, false positive detections due to movements are eliminated. In practice, movements could be identified using a movement detection algorithm, such as [52], [77] and [78]. Automatic movement annotation is beyond the scope of this thesis, and is an active area of research for bed-based sensors [43], [47], [52], [77], [78], both for the purpose of creating a movement-free signal and to use the movements for activity classification.

3. Shallow, fast breathing: Similarly FPs during fast-paced shallow breathing were also not allowed. Study participants were asked to breathe shallowly for approximately $30 \mathrm{~s}$. 
To do so, most participants dramatically increased their breathing rate. Many participants breathed so quickly that the respiband could not respond to the changes in ribcage size and the shallow breathing segments are therefore quite similar to the apnea segments for both types of sensors as shown in Figure 4.22. This type of rapid breathing is unlikely to occur normally. Figure 4.22 shows a data segment for the chest respiband (top) and pressure sensor (bottom). The shallow breathing segment is indicated with a double arrow. Likely, it is not detected as breathing by the respiband due to low pass filtering performed in the instrument.
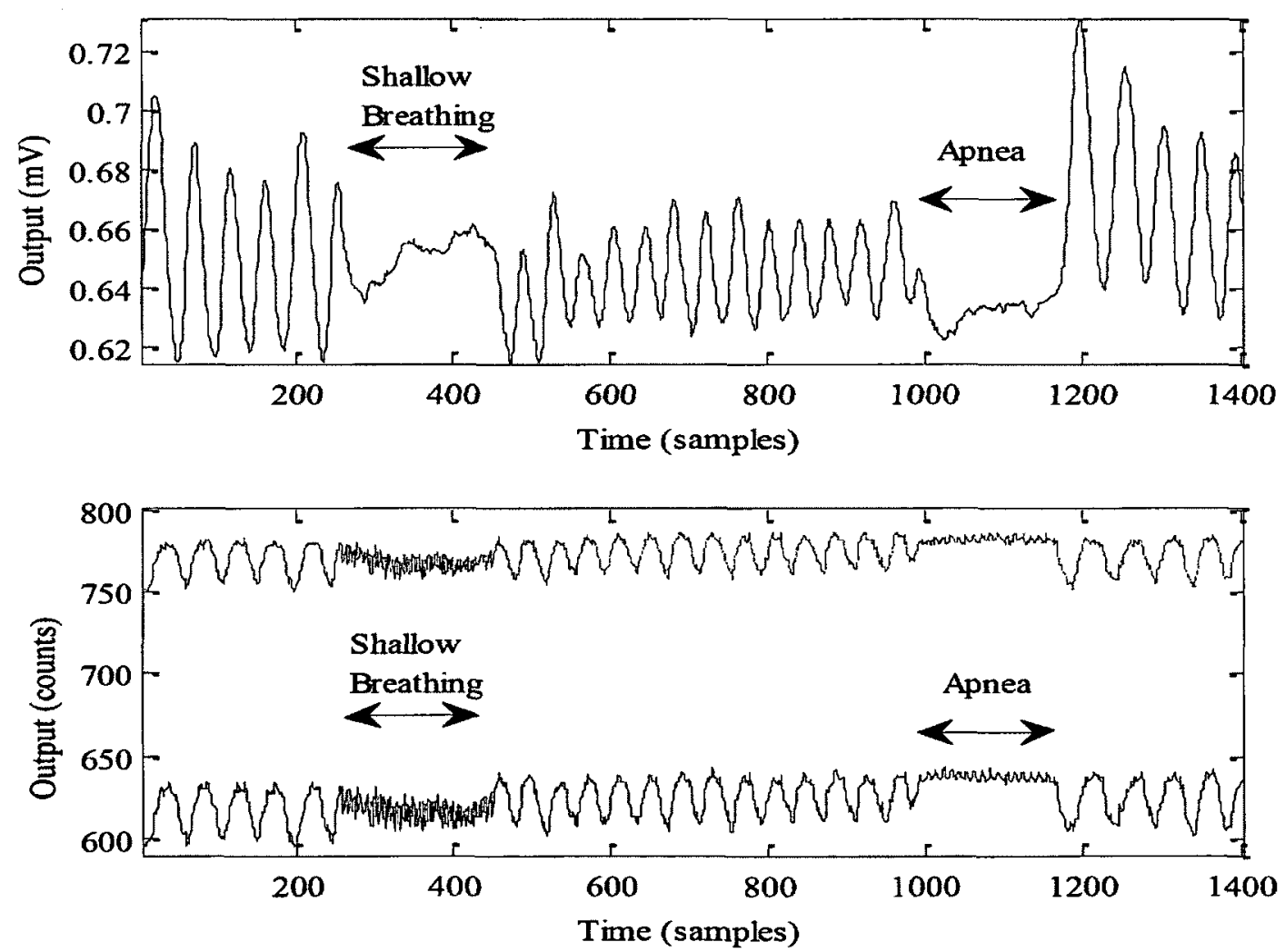

Figure 4.22 Similarity between shallow breathing and simulated $C A$ in respiband signal (top) and in pressure sensor array, two pressure sensor outputs shown (bottom) 
Movements, identified by circles in the sensor output plot, can create false positives as seen in Figure 4.23 where movements occurring at times $t=75,210$ and 390 trigger apnea onset and offset in the classified signal plot. The model detects apneas occurring between two periods of regular breathing as shown in Figure 4.24.

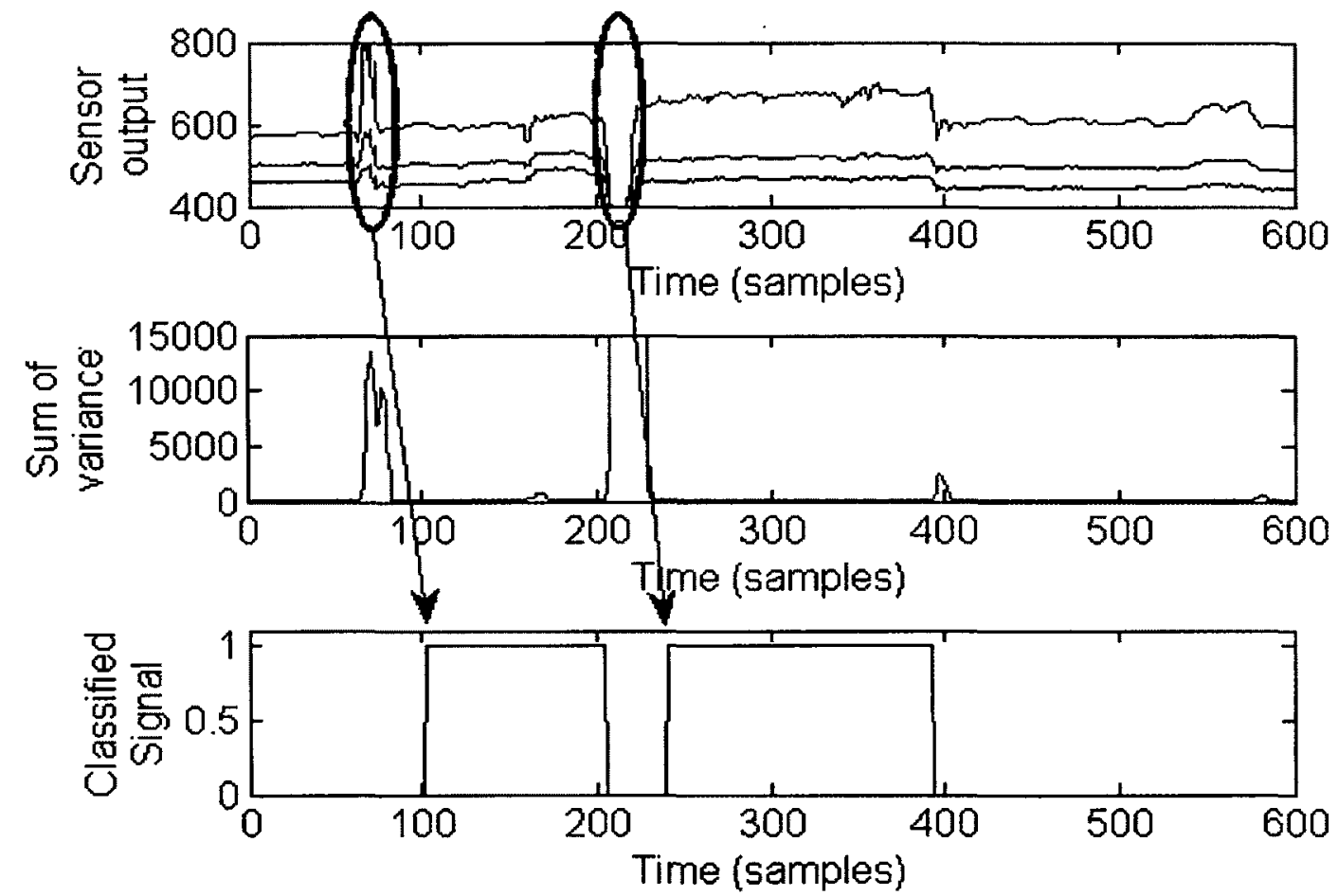

Figure 4.23 Output of three pressure sensors (top plot). Movements, circled in black, create peaks in the variance signal (middle plot), which leads to false positives classifications (bottom plot) 

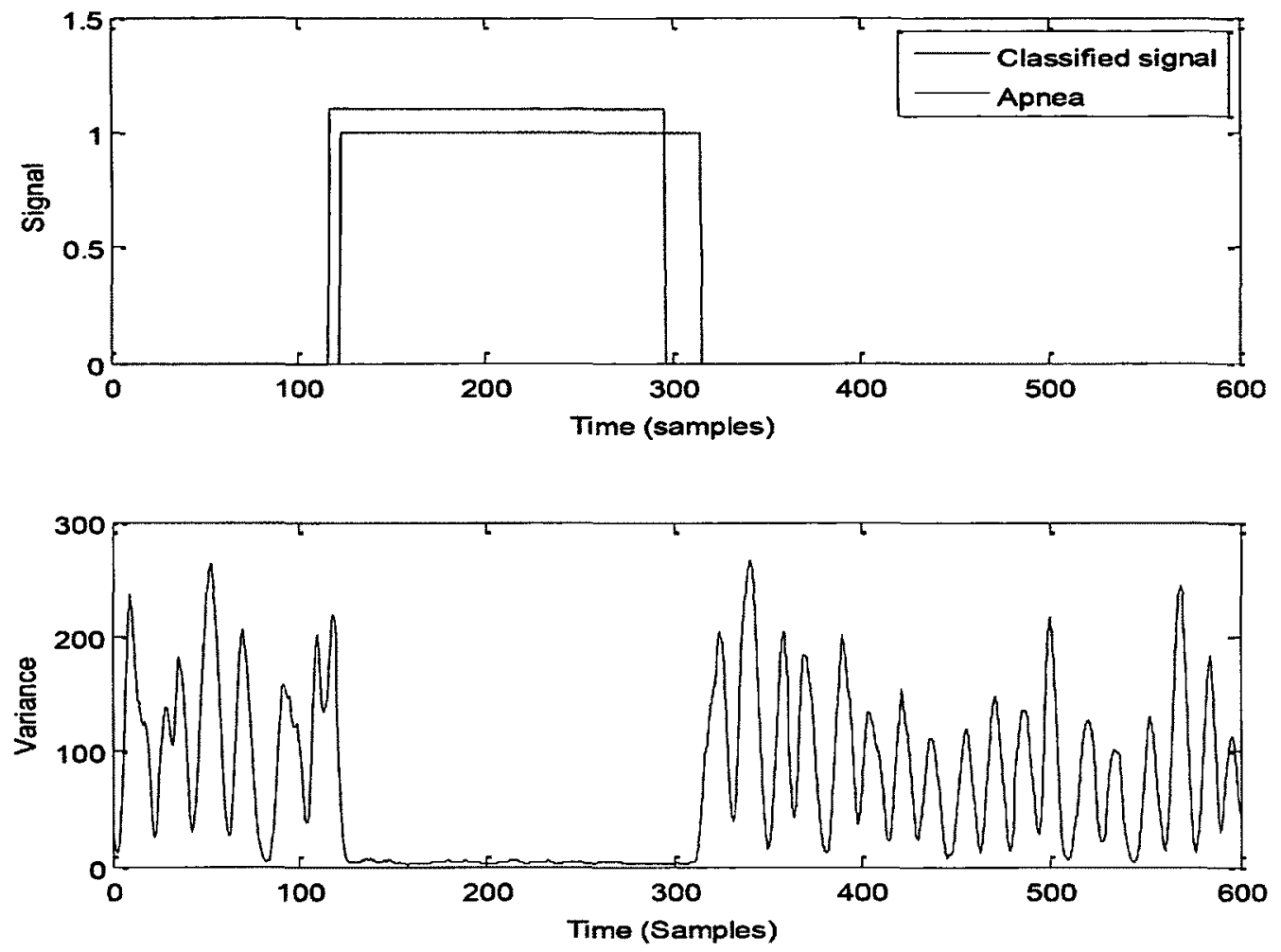

Figure 4.24 Correct apnea detection

\subsection{Results and Verification}

The effect of the $d$ and $t_{1,2}$ parameters on event-based apnea detection is shown by way of the MCC. In cases of event-based classification, the TN metric is not applicable. However, in order to obtain MCC, eq. (4.3) was used to obtain an approximate number of TN epochs by calculating the number of TN segments with a length of 300 samples or 30s. $\mathrm{TN}=\# \mid \mathrm{TN}$ segments $\mid \geq 300$ samples

The test set results were stratified according to position to ascertain the effect of lying position on model parameters. The MCC was calculated for values of $\mathrm{d}$ from 0.1 to 0.99 and values of $\mathrm{N}$ equal to1, 2, 3, and $5 \mathrm{~s}$. Figure 4.25 (left) presents the results for all data, and position separated data. Each data point shows the average MCC value for all 
combinations of $t_{1}$ and $t_{2}$. Figure 4.26 shows the effect of the timing parameters on the MCC curve of the training set.
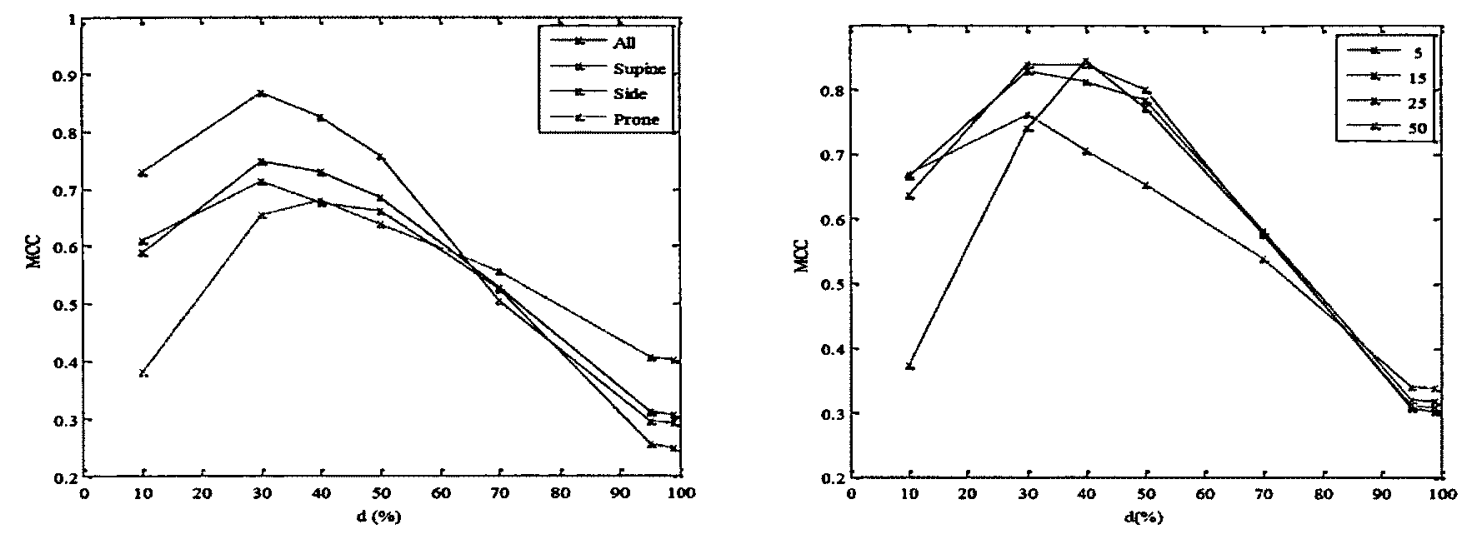

Figure 4.25 Averaged MCC results and effect of threshold d (left) and effect of t1 on MCC for all d (right). Each line represents a different value of $t 1$
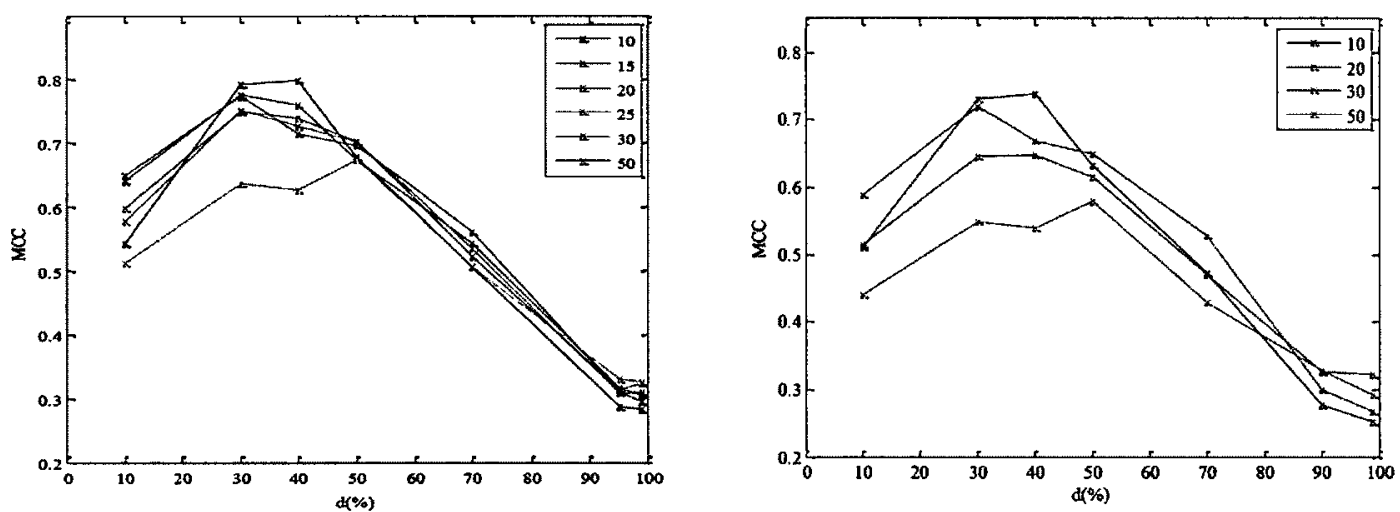

Figure 4.26 Effect of 2 on the MCC. Each curve represents a different value for t2 (left) and effect of window length on MCC (right)

Table 4.7 presents the optimal training set parameters. Parameters were selected to maximize the MCC of the training set. Parameters for the $d(\%)$ column can be read directly from Figure 4.25 (left) as the peak of the respective curves. The Figs. for position-specific $t_{1}, t_{2}$ and $N$ used to obtain the position-specific parameters are not shown. Table 4.8 presents the classification results for the training and test set. Results 
are divided into position and also listed are classification for the entire set, to simulate a context where the position is unknown, and for the 'Position average' which lists the average performance of the training and testing set when the position is known.

Table 4.7 Optimal parameters determined by the training set

\begin{tabular}{|lllll|}
\hline Data & $\mathrm{d}(\%)$ & $\mathrm{t}_{1}(\mathrm{~s})$ & $\mathrm{t}_{2}(\mathrm{~s})$ & $\mathbf{N}(\mathrm{s})$ \\
\hline All & 30 & 1.5 & 3.0 & 1.0 \\
\hline Supine & 30 & 2.0 & 3.0 & 1.0 \\
\hline Side & 30 & 1.5 & 5.0 & 2.0 \\
\hline Prone & 40 & 3.5 & 1.0 & 1.0 \\
\hline
\end{tabular}

Table 4.8 CA classification results for training, testing and validation set

\begin{tabular}{|c|c|c|c|c|c|c|c|c|}
\hline & PPV & $\mathrm{TP}$ & FP & FN & Sens & Spe & FPR & $\mathrm{MCC}$ \\
\hline \multicolumn{9}{|l|}{ Training Set } \\
\hline All & 1 & 7 & 0 & 2 & 0.78 & 1.00 & 0.00 & 0.88 \\
\hline Supine & 1 & 2 & 0 & 1 & 0.67 & 1.00 & 0.00 & 0.81 \\
\hline Side & 1 & 3 & 0 & 0 & 1.00 & 1.00 & 0.00 & 1.00 \\
\hline Prone & 1 & 3 & 0 & 0 & 1.00 & 1.00 & 0.00 & 1.00 \\
\hline $\begin{array}{l}\text { Position } \\
\text { average/total }\end{array}$ & 1 & 8 & 0 & 1 & 0.89 & 1.00 & 0.00 & 0.94 \\
\hline \multicolumn{9}{|l|}{ Test Set } \\
\hline All & 0.75 & 12 & 4 & 5 & 0.71 & 0.99 & 0.01 & 0.72 \\
\hline Supine & 0.43 & 3 & 4 & 2 & 0.60 & 0.99 & 0.01 & 0.49 \\
\hline Side & 1 & 5 & 0 & 1 & 0.83 & 1.00 & 0.00 & 0.91 \\
\hline Prone & 1 & 6 & 0 & 0 & 1.00 & 1.00 & 0.00 & 1.00 \\
\hline $\begin{array}{l}\text { Position } \\
\text { average/total }\end{array}$ & 0.78 & 14 & 4 & 3 & 0.81 & 1.00 & 0.00 & 0.80 \\
\hline \multicolumn{9}{|l|}{ Validation Set } \\
\hline All & 0.8 & 4 & 1 & 3 & 0.57 & 1.00 & 0.00 & 0.79 \\
\hline Supine & 0.33 & 1 & 2 & 2 & 0.33 & 0.99 & 0.01 & 0.32 \\
\hline Side & 1 & 4 & 0 & 1 & 0.80 & 1.00 & 0.00 & 1.00 \\
\hline Prone & 1 & 4 & 0 & 0 & 1.00 & 1.00 & 0.00 & 1.00 \\
\hline $\begin{array}{l}\text { Position } \\
\text { average/total }\end{array}$ & 0.78 & 9 & 2 & 2 & 0.71 & 1.00 & 0.00 & 0.77 \\
\hline
\end{tabular}




\subsubsection{Effect of Parameters on ROC Curve}

The effect of the apnea model parameters on the classifier properties were also assessed by means of the ROC curve. Figure 4.27 shows the ROC curve for a change in the threshold $d$, with the $d$ values indicated along the curve. Parameter values are labeled along the line clearly show that increasing $d$ increases the number of events classified as positives. This parameter shows the greatest change in area under the $\mathrm{ROC}$, and provides much of the flexibility in the model. A decrease in $t_{1}$ leads to a slightly higher false alarm rate and slightly lower TP rate until a $t_{2}$ value of $1.5 \mathrm{~s}$, where any further decrease reduces the false alarm rate. Increasing the value of $t_{2}$ increases the detection rate of events, whether TP or FP. Lower values of $t_{2}$ may be preferable as higher ones increase the risk of not finding successive apneas that occur within a few seconds.

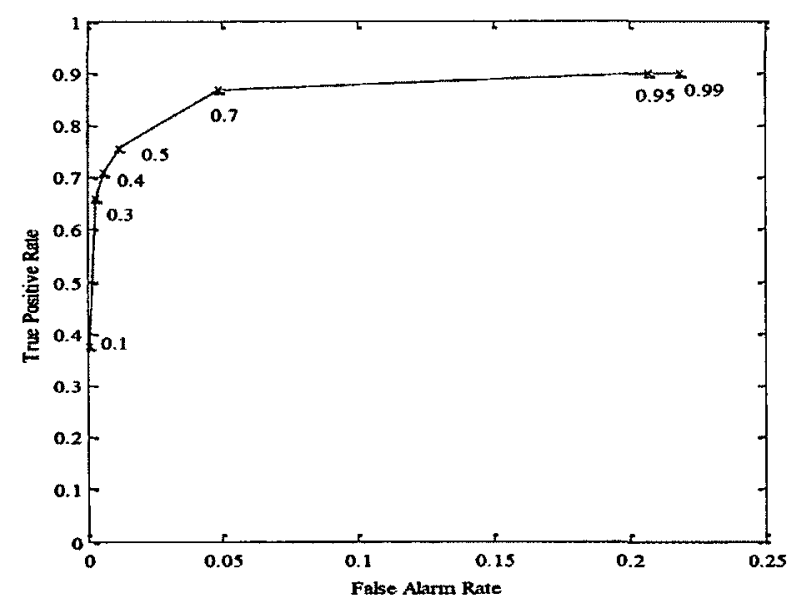

Figure 4.27 Effect of the threshold $d$ on the ROC curve for CA detection

\subsubsection{Effect of Mattress Type}

The test and validation sets were combined and their results compared by mattress type. Figure 4.28 shows a bar graph of MCC and PPV across each of the mattress types. In 
general, all mattress types are in a range above 0.5 for both PPV and MCC. Of the three types, the coil mattresses tend to be slightly worse at transmitting the apneas.

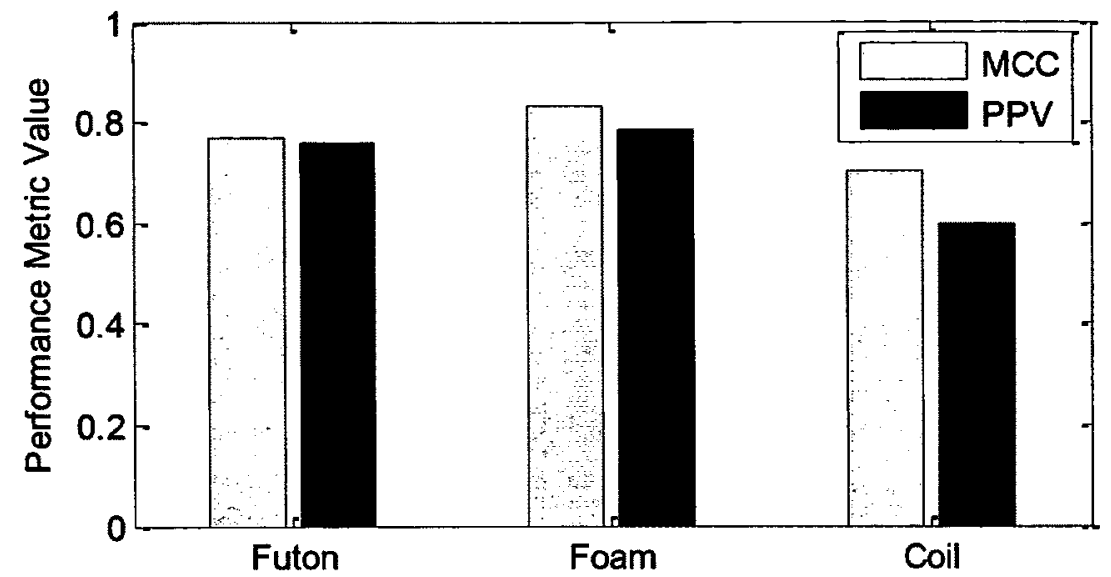

Figure 4.28 MCC and PPV according to mattress type

\subsubsection{Discussion}

Event classification can be more informative than epoch classification since it allows for precise localization and measurement of events as required for traditional PSG. The use of relative thresholding allowed simulated CAs to be detected on different mattresses. Relative thresholding is a technique that successfully detects apneas from a variety of mattresses because it is not greatly affected by the breathing amplitude or noise in the signal. This method also does not require much pre-conditioning or filtering of the raw signal. However it relies on proper movement annotation. Manual annotation is possible for short files as in these controlled experiments.

The $\mathrm{d}$ threshold parameter has the most impact on the model's ability to correctly identify simulated CAs, as demonstrated by the ROC curve of Figure 4.28 . It is the most important factor for tuning the FP and TP values. The present results were tuned to maximize the MCC by selecting a threshold $d$ of $30 \%$. Selecting a larger value increases 
the FP and TP values, while selecting a smaller $d$ can be used to decrease the FP rate which is useful for applications with strict limits on the false alarm rate.

The subjects were not constrained to a certain placement on the mattress, so that limb, spinal cord, torso, and shoulder position could vary with respect to sensor locations. The algorithm is expected to be robust to subject placement. Lying position has a slight effect on the MCC curves as shown in Figure 4.25. All curves peak between a $30 \%$ and $60 \%$ value for the threshold $\mathrm{d}$. Above the peak, the number of FP is quite high, while below, the number of detected apneas decreases. The effectiveness of the FP reduction strategy is apparent in the low FP event rate; a measure which is important in biomedical monitoring. Many false apneas were detected as very long ( $\geq 60 \mathrm{~s}$ ) segments. These often occurred as the result of a small muscular movement which created a peak in the moving variance signal, triggering the $t_{1}$ and $d$ from the apnea model. Removing these false apneas increases both the MCC and PPV of the classifier. The sensitivity was highest in side and prone positions in all data sets. In some cases, the BCG effect was particularly pronounced in the supine position, and this could have an effect on the detection algorithm.

The validation set results are consistent with those of the test set, suggesting that there is may be a generalization of the parameters found with the test set to different body types and weights, though more data is required to determine if the effect is statistically significant. The position based MCC result was lowest for the supine position. In the data used here, this position tends to have the lowest signal to noise ratio, which may affect the model's ability to correctly classify apneas. The parameters determined from the training set were applied to the test set and produced classification results of $0.78 \mathrm{PPV}$ if 
the bed occupant's position is known, and $0.75 \mathrm{PPV}$ if position is unknown. This is an improvement over sample [2] and more recent segment classification [3]. Independent of position awareness, the classifier displayed excellent specificity, indicating that few if any non-apnea events were classified as apneas.

In 2010, Shin et al. detected simulated CAs using a hard-coded threshold on the $3 \mathrm{~s}$ moving variance signal form an air mattress which collected pressure information from beneath the chest and abdomen region [79]. They obtained a PPV of 0.93 and a sensitivity of 0.88 with their classifier which used 15 simulated CAs and 15 epochs of normal breathing. One advantage of using a relative threshold is good classifier performance across mattress types, allowing the users of a biomedical monitoring system to select their own mattress. The relative threshold may also produce better results for weight and height variations between users.

\subsection{Conclusions}

To improve sample by sample classification, which is quick though not sufficiently discriminating for clinical applications, moving windows were applied. MA windowing improved the classification sensitivity, while WE improved the specificity. The duration of apnea detections in this controlled experiment correlated well with actual values. The third experiment demonstrated that using data-driven contextual information, to determine the person's sleeping position can improve classification results by changing parameters in the algorithm. Unlike in hospitals, where bedding is standardized, the different mattress types used in smart homes poses an additional challenge to successful unobtrusive monitoring. The use of an adaptive threshold allows features of signals to be detected under a variety of different conditions. If the patient-driven context is provided, 
it allows for mattress type as an input and may tailor the algorithm by changing a parameter. Hard coded parameters can be more useful for data with fully annotated movements and where the instrumentation and participant is static. The effect of successive algorithm improvements on classification results through sensor fusion and context-awareness in shown in Table 4.9.

Table 4.9 CA classification results through algorithm evolution

\begin{tabular}{|c|c|c|c|c|c|}
\hline & Multisensor & MA & WE & $\begin{array}{l}\text { Apnea Model } \\
\text { Position Known* }\end{array}$ & $\begin{array}{l}\text { Apnea Model } \\
\text { Position Unknown: }\end{array}$ \\
\hline Sens & 0.54 & 0.82 & 0.75 & 0.71 & 0.57 \\
\hline Spe & 0.96 & 0.69 & $\overline{0.92}$ & 1.00 & 1.00 \\
\hline FPR & 0.05 & 0.31 & 0.08 & $<0.01$ & 0.00 \\
\hline MCC & 0.48 & 0.30 & 0.45 & 0.8 & 0.79 \\
\hline
\end{tabular}

The under mattress pressure sensor does not directly measure the airflow or respiratory effort, though it captures movement information transmitted through the mattress by a person lying on it. The agreement between the actual and detected apneas in this work indicates a relationship between events that can be extracted from the pressure sensor and breathing patterns. 


\section{Chapter 5: \\ Central Apnea Clinical Tests}

\subsection{Introduction}

This chapter presents the small scale clinical tests performed for CA detection in contextaware sleep monitoring ${ }^{7}$. At the West Ottawa Sleep Center in Ottawa, ON, data was collected simultaneously from all PSG sensors and from two under the mattress pressure sensor arrays. Ethical approval was obtained from the sleep laboratory and from Carleton University. To arrive at a set of six patients, the author intended to look at data from consecutive patients referred for overnight laboratory polysomnography who would consent to have an under mattress sensor pad running simultaneously with a baseline polysomnogram. After scoring, if subjects had obstructive apneas, or Cheyne-Stokes respiration (CSR), or presented with a BMI over 35 they would be excluded. In many cases with higher BMI $(>35)$ most of the sensors were saturated throughout the recording, making any analysis more challenging with the particular sensor used. Section 5.2 describes the data collection at the sleep laboratory and Section 5.3 presents the proposed approach. Section 5.4 presents the results and validation of the apnea detection

\footnotetext{
${ }^{7} \mathrm{~A}$ part of this chapter is published in [5] with validation published in [6]. Clinical aspects of the study are presented in [7].
} 
on the PSG data followed by a discussion of the results in Section 5.5. The chapter concludes with Section 5.6.

\subsection{Data Collection and Preparation}

Patients regularity scheduled to undergo a baseline polysomnogram were given the option of participating in the study. From the patient's perspective, participation in the study involved no extra effort, tests or signal calibrations than that required by the polysomnogram itself as the sensor is unobtrusive and had been previously installed under the mattress. For the technologist acquiring the polysomnography signals, patient participation in the study required the extra connection of two RJ 45 type cables into a data acquisition box with colour coded outlets located at the patient bedside. Data collection was performed by serial connection for one sensor array and by USB connection for the other sensor to the laptop located underneath the bed as described in Townsend et al. [10]. Two rooms were each equipped with two under the mattress pressure sensor arrays and one logging computer (Compaq Armada/Dell Inspiron) located underneath the bed which logged data 24 hrs a day. The logging program created one new comma separated-values file every six hours with one row recording the pressure at each sensor element approximately every $0.1 \mathrm{~s}$. The sensor arrays remained in the same position for the entire data collection period and their placement underneath the mattress did not interfere with daily linen changing. Two pressure sensor arrays were placed beneath the double size coil mattress $(137 \mathrm{~cm} \times 190 \mathrm{~cm}), 10 \mathrm{~cm}$ apart to maximize

sensor coverage of the torso area of the participants and were further secured to a sheet of plywood with tape to ensure they remained in position. 


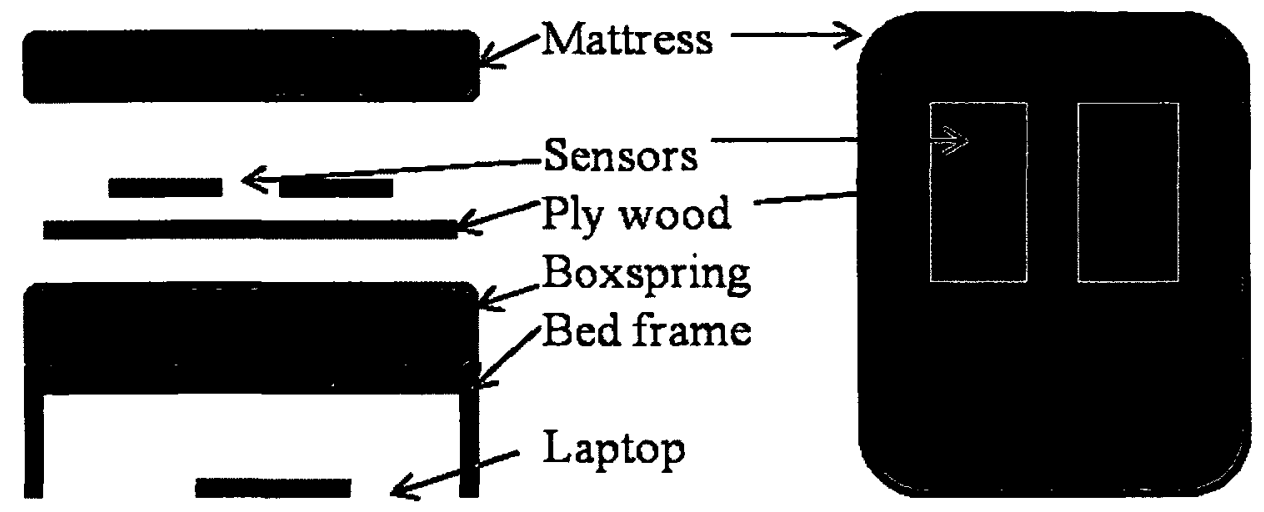

Figure 5.1 Instrumentation setup for data collection in sleep laboratory showing sensors secured to piece of plywood located between mattress and box spring

The sensors were manually connected to the data acquisition system for consenting patients and the $\log$ files were collected bi-weekly by M. Holtzman and/or D. Townsend. The data collection yielded complete datasets for over forty patients, though not all presenting with CAs. Incomplete physical connection of the sensor's cable with the data acquisition box and disconnection of the laptop from the power outlet was responsible for most cases of incomplete and missing data logs.

For each consenting participant for whom pressure array data had been successfully collected, the West Ottawa Sleep Center provided the following files:

1) an .EDF file containing the PSG signals for all sensors used (except video camera information). The EDF (European Data Format) is the standard format for EEG and PSG data. It contained the raw sensor signals.

2) a .txt file containing a time stamped annotated list of all epochs, events, event durations and sleep stage information as well as the time and description of the biocalibration events. This second file, known as the Event Report situates each event three ways: 

a. in the epoch in which it occurred
b. its start time in relative seconds since the beginning of the recording
c. its time relative to the sleep lab's computer.

Biocalibration allows the technician to scale and apply digital filters to the signals as well as assess electrode placement. Most patients were asked to perform tasks such as opening and closing the eyes, moving the legs, holding a breath for 5-10 $\mathrm{s}$ and hyperventilate for 5s. When performed, these last two breathing events were used to align the pressure array data to the .txt file. Each PSG file was scored by one of the West Ottawa Sleep Center's four polysomnographers who are registered with the AASM. The polysomnographers interpreted the overnight recording using the guidelines published by the AASM.

The EDF file, Event Report and pressure data log were aligned according to the following steps. Most processing was performed in MATLAB (R2009a):

1. Individual signals from the EDF file were exported to ASCI then converted to integer files. Signals were then scaled using the calibration information saved in the file header and down sampled to $10 \mathrm{~Hz}$.

2. Using the Event Report (computer time column), the relevant pressure array sensor log files were retrieved. Data from both arrays was combined and missing samples were interpolated. Two or three 6 hour-long files were associated with each overnight recording depending on its length and start time.

3. A script was created to read the Event Report and create a single-outcome file for body position and apnea (central and obstructive when available). 
4. The down sampled PSG and pressure array signals (all at $10 \mathrm{~Hz}$ ) were plotted and visually aligned by locating the exact start times of the biocalibration events listed in the Event Report.

5. Lastly, extracted PSG signals and single-event signals were padded to the same length as the pressure array files for ease of processing.

\subsection{Proposed Approach: Amplitude Limit}

The amplitude limit approach is presented in this chapter to validate the role of the pressure sensor array in CA detection for sleep laboratory patients. The modifiable parameters in the algorithm are the maximum allowable amplitude ' $a$ ' and the length of the sliding window ' $\mathrm{S}_{\mathrm{L}}$ '. These parameters allow the tailoring of a model to determine if there is a set of parameters that produces optimal results for each file to make a screening or patient specific tool for long-term monitoring tool.

Figure 5.2 presents a block diagram of the proposed amplitude limit approach. The method allows a limit to be set on the amplitude of the segment of moving variance found in the interval defined by the window. A mean loading value is removed from the raw data, and then the moving variance of each individual sensor's output is calculated. These moving variance signals are then summed together to produce a fused signal. After selection of the amplitude parameter, the sliding window is applied to the moving variance signal. If the peak amplitude of the signal falls under the limit, the portion of signal in the window is classified as 'apnea', otherwise it is 'non-apnea'. 


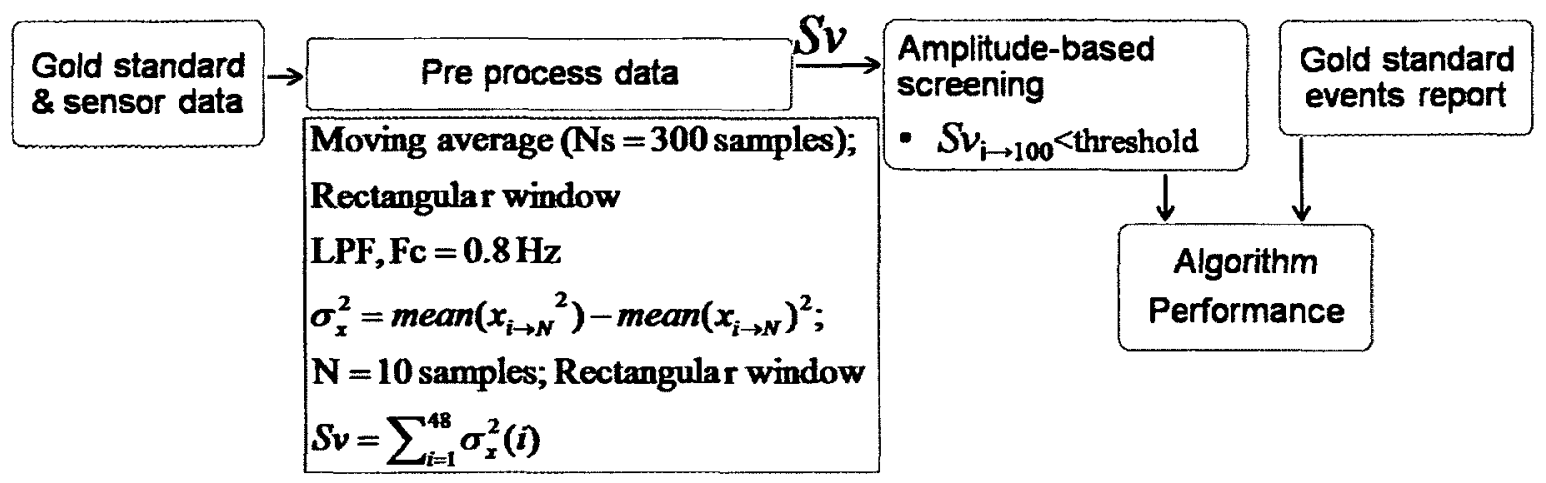

Figure 5.2 Block diagram of data analysis for the amplitude limit approach

Figure 5.3 shows how the model is applied to a variance signal. Top plot shows the variance signal for a minute long segment, while the bottom plot focuses on $t=12.5 \mathrm{~s}$ to $t$ $=37.5 \mathrm{~s}$. The horizontal arrow shows a $10 \mathrm{~s}$ long window in which the maximal amplitude is calculated. The calculated value, denoted by ' $a$ ' in the figure, is compared to the specified threshold to determined if the segment fits the criteria for CA classification.

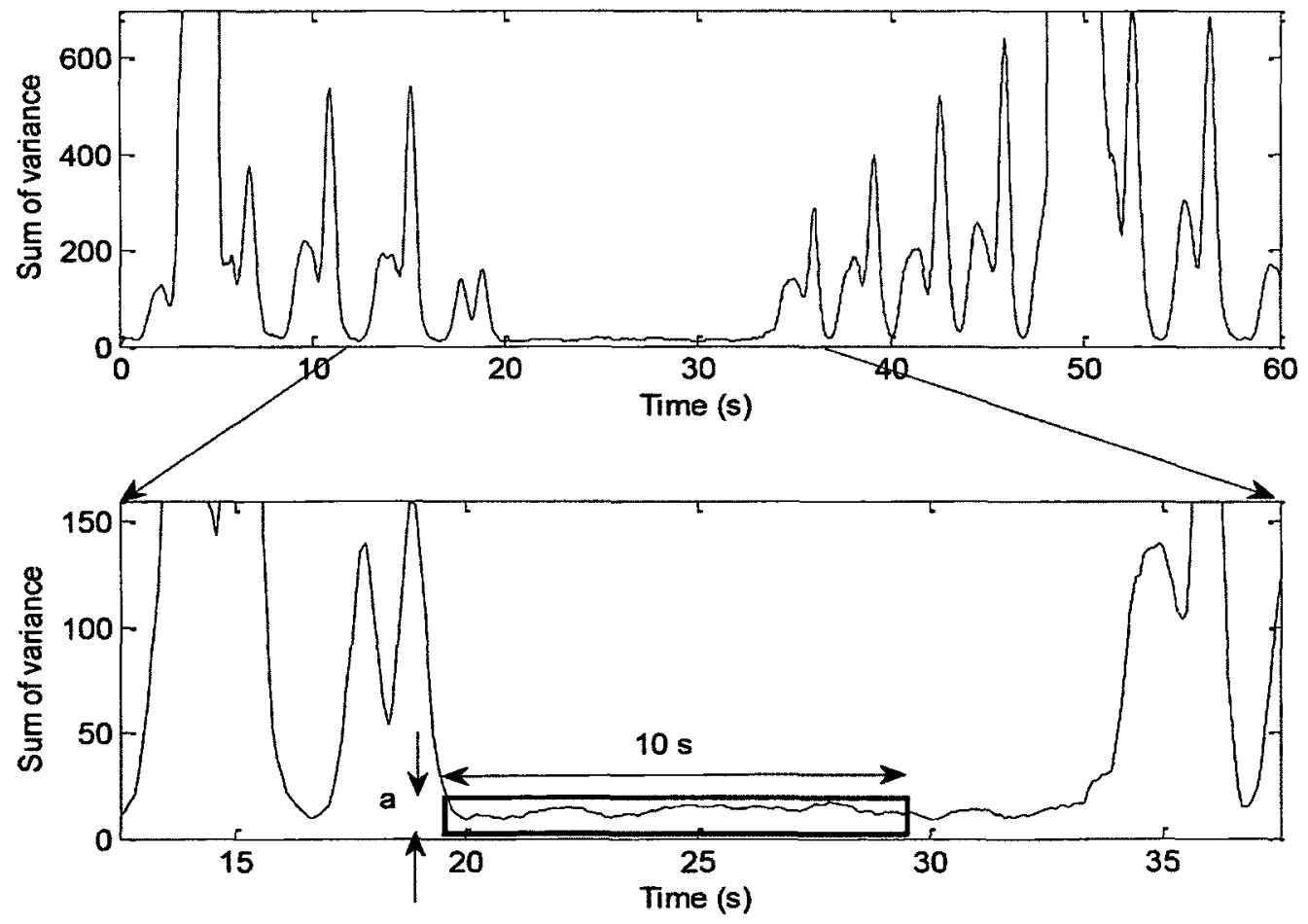

Figure 5.3 Central apnea model applied to moving variance signal, where ' $a$ ' is the amplitude of the variance contained within the $10 \mathrm{~s}$ sliding window 
The algorithm was applied to the fused sensor pressure array, and to four PSG signals: the two respibands, the airflow sensor and the thermistor. The blood oxygen saturation $\left(\mathrm{SpO}_{2}\right)$ signal was independently processed to determine the presence of a desaturation $\geq 1 \%$ during periods detected as apneas by the algorithm on any signal.

Multiple scenarios were analyzed to observe and meet the following goals:

1) Confirm the ability of the pressure sensor array to detect CAs in PSG data from multiple patients.

2) Compare the pressure sensor array to PSG sensors without expert interpretation.

3) Examine if the inclusion of a desaturation criterion on the CA scoring guideline affects the pressure sensor array and respibands classification similarity.

\subsection{Results and Verification}

\subsubsection{Pre-Validation Work}

The algorithm was implemented and controlled experiments were conducted with eight participants to determine the effect of the number of samples in the moving variance window, amplitude limit and the impact of mattress type on the proposed algorithm. Figure 5.4 shows the probability of detection rising quickly to over $50 \%$ with an amplitude limit of 1 , and then rising at a slower pace in all four curves. As the length of the moving variance window increases from $1 \mathrm{~s}$ to $5 \mathrm{~s}$, fewer apneas are detected at each amplitude value. Figure 5.5 shows the probability of detection for data divided by mattress type. The moving variance was calculated with a 1s window only. Each mattress type produced a distinct curve with the coil mattresses reaching $100 \%$ detection at a very low limit. 
The preliminary results suggest that the detection of CAs is possible under these controlled conditions. The most important condition is that the variance signal during the simulated apnea be free of movement. The probability of apnea detection generally decreased as the window length increased indicating the transition zone between breathing and apnea became longer, thus reducing the length of the apnea's flat section. Shorter apneas, measuring closer to the $10 \mathrm{~s}$ limit, did not get detected until the limit on the apnea was high enough to consider the curves at either side. Shortening the sliding window would help determine which FN occur as a result of length and which remain undetected due to their amplitude

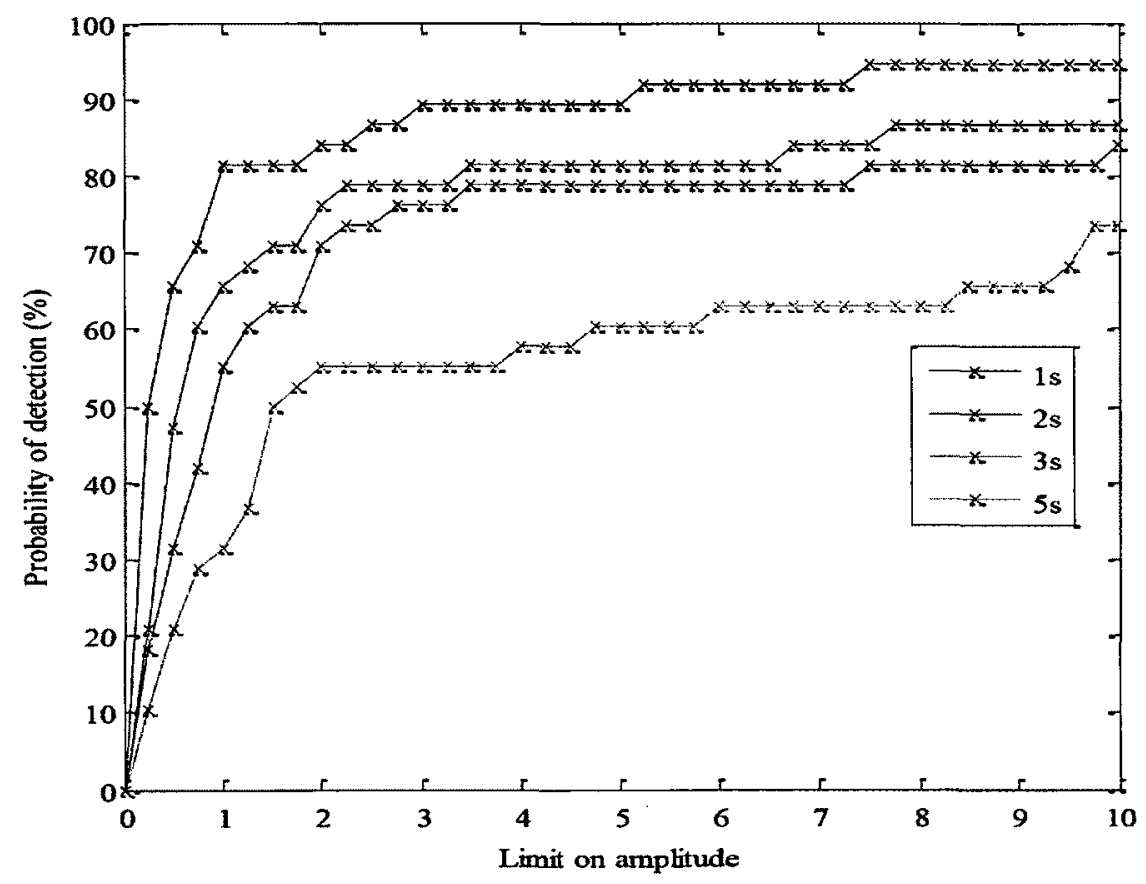

Figure 5.4 Effect of the amplitude limit on the probability of CA detection for changes in window length $\mathbf{N}$ 


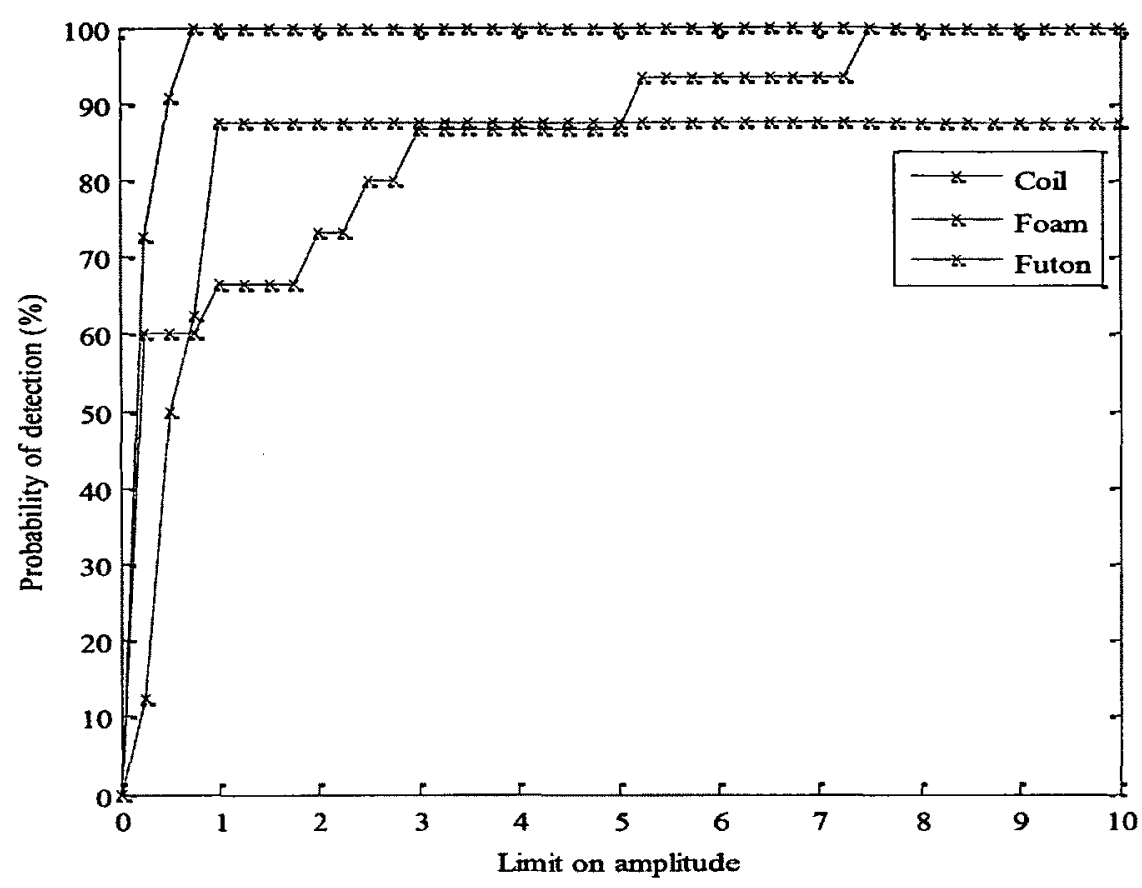

Figure 5.5 Effect of mattress type on the probability of CA detection ( $N=1 \mathrm{~s}$ )

The size of the sliding window could be reduced to capture apneas which are shorter or which may be affected by forms of data processing including down sampling. This amplitude-based screening offers a high probability of detection for simulated central apneas on coil and futon mattresses even at low amplitude limits. The proposed approach was applied to the validation data.

\subsubsection{Data Synchronization}

The biocalibration is performed at the beginning of a polysomnogram, and repeated when electrodes fall off and are reapplied. The respiband calibration lends itself well to a sensor synchronization task. Figure 5.6 shows the chest and abdomen PSG respiband signals during the relevant portion of the biocalibration, while Figure 5.7 shows the output of the pressure sensor array during the same movements. 


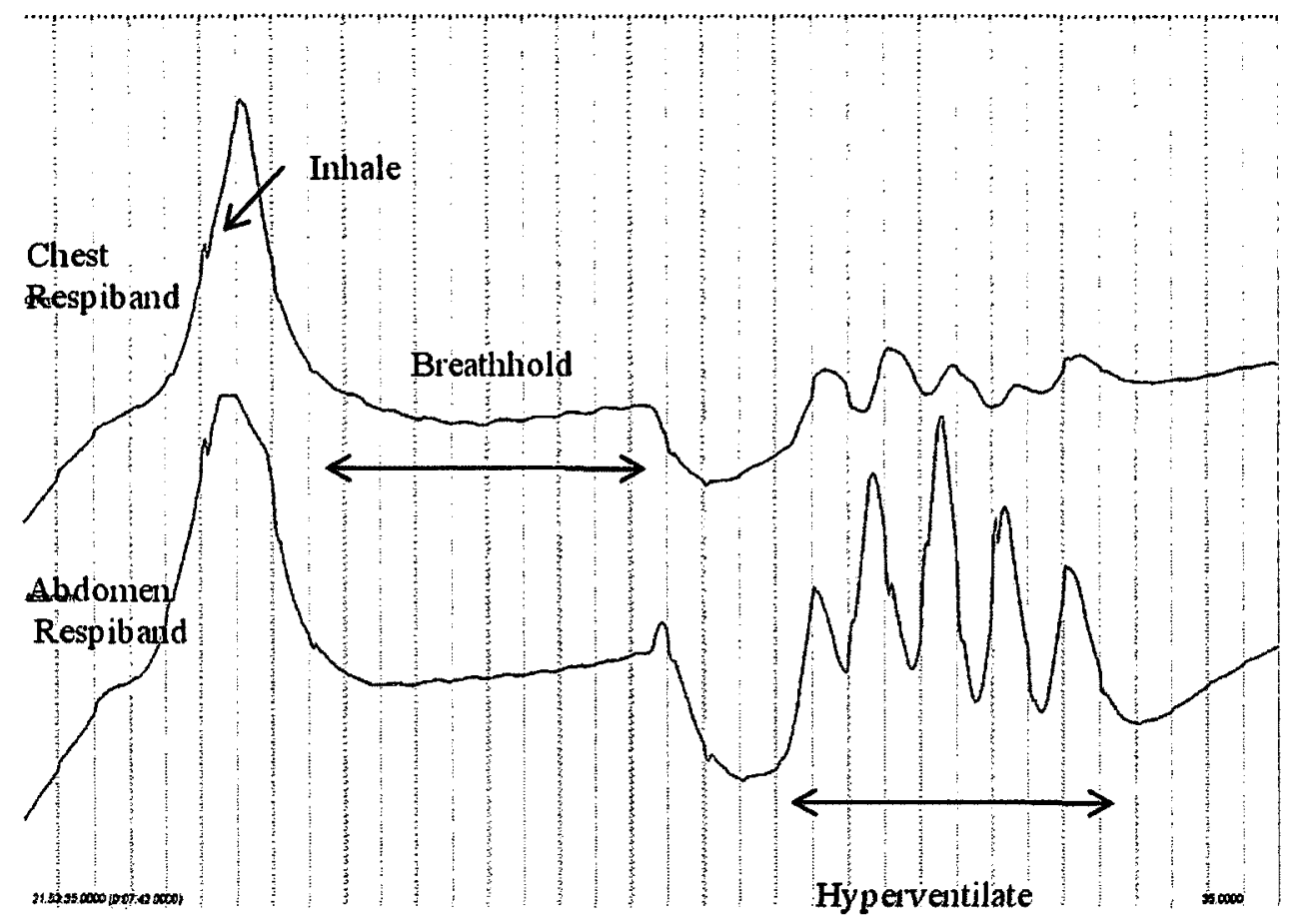

Figure 5.6 PSG respiband signals during biocalibration ( $35 \mathrm{~s}$ of signals shown)

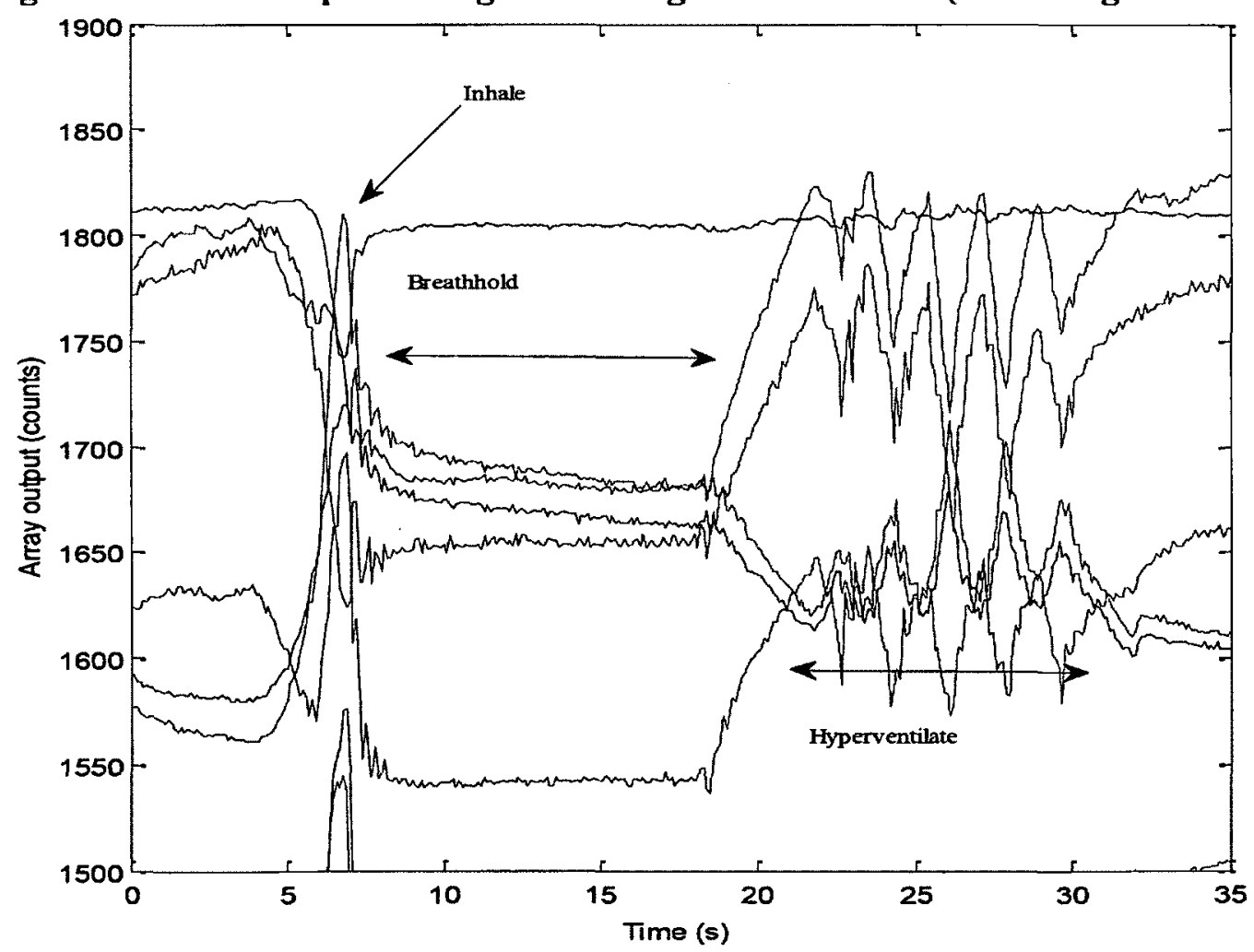

Figure 5.7 Output signal of selected array sensors during biocalibration of respibands 
The inhalation peak is sharper in the chest respiband while the peaks of respiration during the hyperventilation segment are sharper in the abdominal respiband. In Figure 5.7, the top (blue) sensor contains information about the inhalation event suggesting that this sensor may be minimally loaded, while the 5 other sensors have similar shapes and display the three biocalibration movements.

Table 5.1 lists the anthropometric patient information including patient number $(\mathrm{Pt})$, body mass index (BMI) and AHI of the eight analyzed files ready.

Table 5.1 Anthropometric patient information

\begin{tabular}{|llllll|}
\hline Pt\# & Gender & cm/in & Kg/lb & BMI & AHI \\
\hline 1 & Male & $182 / 6^{\prime} 0^{\prime \prime}$ & $116.5 / 256$ & 34.2 & 24.4 \\
\hline $\mathbf{2}$ & Female & $164 / 5^{\prime} 4^{\prime \prime}$ & $81.4 / 179$ & 31.0 & 0.3 \\
\hline 3 & Male & $178 / 5^{\prime} 10^{\prime \prime}$ & $77.4 / 170$ & 24.4 & 3.1 \\
\hline $\mathbf{4}$ & Male & $187 / 6^{\prime} 2^{\prime \prime}$ & $86.4 / 190$ & 24.7 & 1.6 \\
\hline $\mathbf{5}$ & Female & $132 / 4^{\prime} 4^{\prime \prime}$ & $45.4 / 100$ & 26.1 & 0.3 \\
\hline 6 & Female & $167 / 5^{\prime} 6^{\prime \prime}$ & $52.3 / 115$ & 18.8 & 0.8 \\
\hline 7 & Male & $170 / 5^{\prime} 7^{\prime \prime}$ & $110.4 / 243.0$ & 38.2 & 57.9 \\
\hline $\mathbf{8}$ & Male & $167 / 5^{\prime} 6^{\prime \prime}$ & $108.8 / 239$ & 39.0 & 9.4 \\
\hline
\end{tabular}

Figure 5.8 below shows the output of selected pressure sensors during a central apnea in a polysomnography patient. The CA occurs between $\mathrm{T}=23 \mathrm{~s}$ and $\mathrm{T}=40 \mathrm{~s}$ and is scored as just over $10 \mathrm{~s}$ in length. 


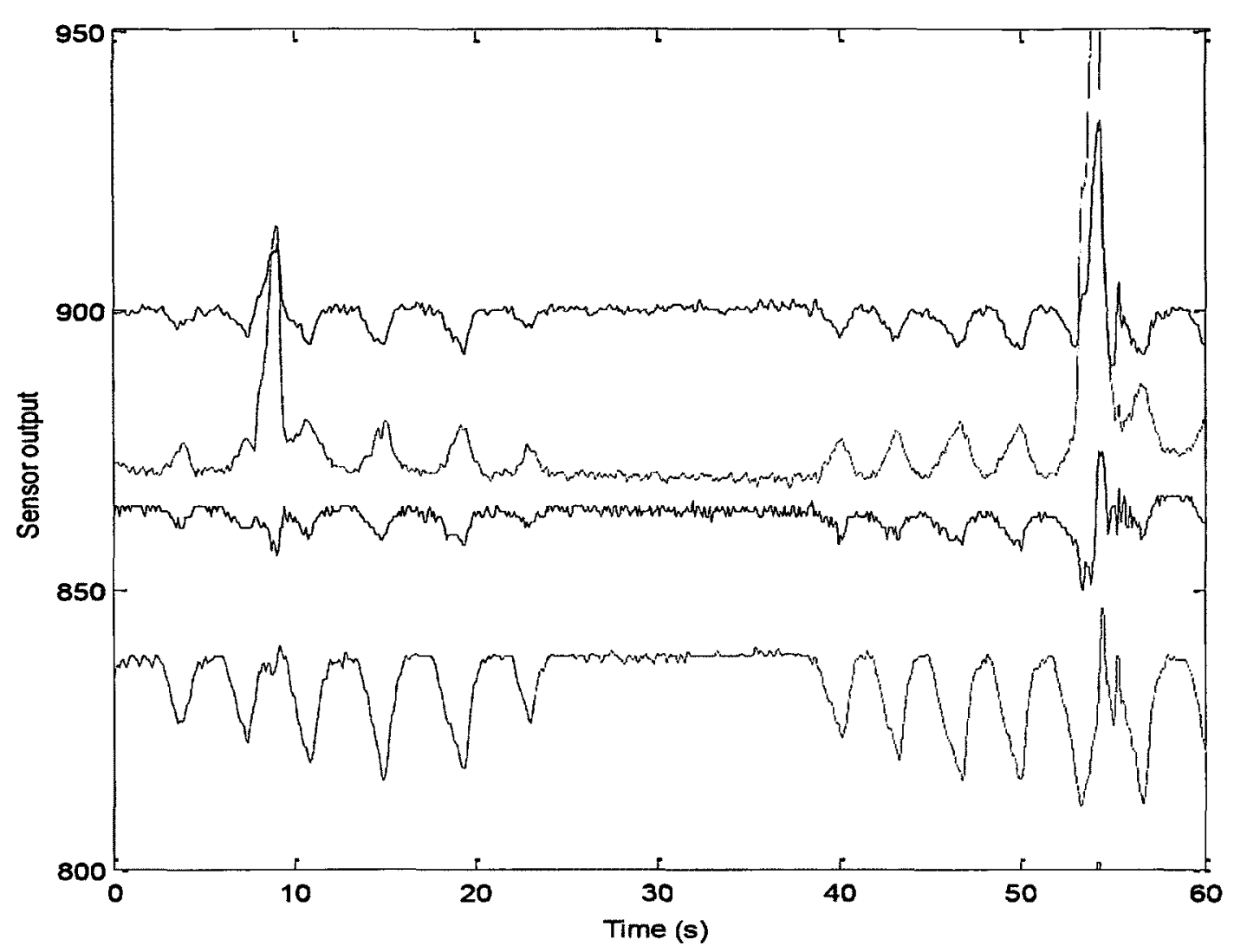

Figure 5.8 Central apnea $(t=23 \mathrm{~s}$ to $t=40 \mathrm{~s})$ detected during a polysomnogram as captured by selected pressure sensors.

Figure 5.9 shows some of the sleep laboratory signals. The respibands, oronasal air pressure and $\mathrm{SpO}_{2}$ sensors are used in the identification of a CA. In the top plot the chest and abdomen respiband signals show the $\mathrm{CA}$ occurring as a relatively still section between $\mathrm{t}=20 \mathrm{~s}$ and $\mathrm{t}=40 \mathrm{~s}$. The air pressure signal from the middle plot also clearly shows the CA, while the bottom plot shows a desaturation occurring during, and one occurring after the CA. 

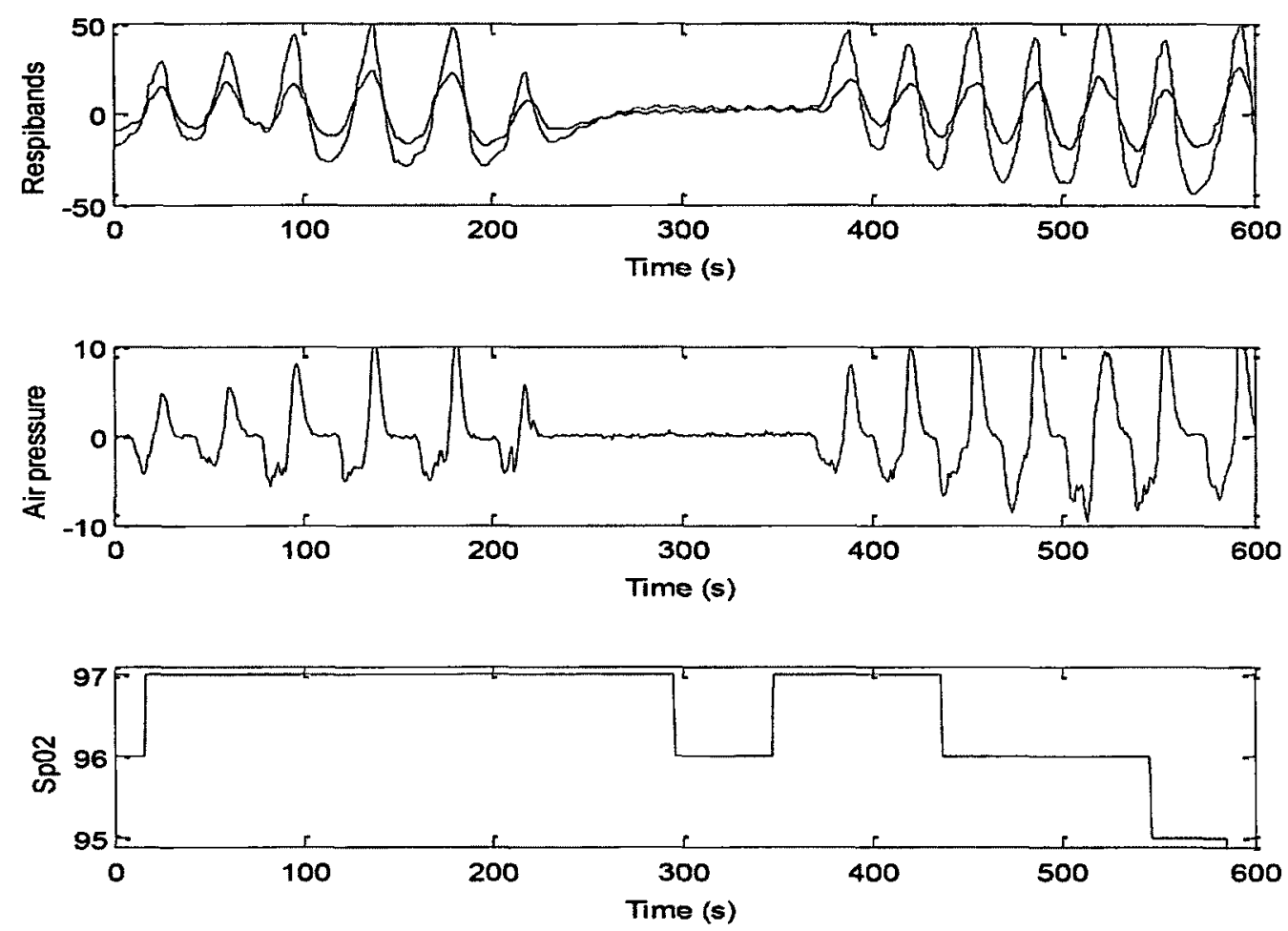

Figure 5.9 Central apnea in validation data as captured during the polysomnogram by the respiration band signals, air pressure sensor, and $\mathrm{SpO}_{2}$ sensor

\subsubsection{Validation of CA Detection}

The CA detection algorithm was applied to the complete night PSG recordings and to the pressure sensor array data for the patients listed in the table above. $100 \%$ of PSG epochs and the corresponding epochs in the sensor array data were used in this chapter's analysis. No epochs were discarded. The a and $S_{L}$ parameters were tailored for each participant to provide optimal detection results. The first objective was to determine if the pressure sensor array can detect CAs in PSG data. To meet this objective, the classified pressure sensor array data was compared to the expert interpretation, or scoring. 
Table 5.2 presents the epoch-based comparison of the scoring to the apneas detected by the pressure sensor array. Cohen's kappa value $(\kappa)$ is reported for each file. Both classified signals were divided into 30s epochs. Any epoch containing more than $1 \mathrm{~s}$ of an apnea was classified as positive. Remaining epochs were classified as negative.

For readability purposes in the tables 'respibands' was shortened to 'Respi', and the processed pressure sensor array as 'Array' in all tables of this chapter. The first column in the table below indicates the date of the study and the next indicates the number of CAs and the position in which they occurred. The positions include lateral (L), prone (P) or supine (S). The Array column lists the total number of apneas as detected by the algorithm and the three remaining columns rate its classification properties.

Table 5.2 CA classification results for the pressure sensor array

\begin{tabular}{|llllll|}
\hline Pt\# & $\begin{array}{l}\text { Scoring } \\
(\# C A)\end{array}$ & $\begin{array}{l}\text { Array } \\
(\# C A)\end{array}$ & Sens & Spe & $\kappa$ \\
\hline 1 & $1 \mathrm{~S}$ & 1 & 100 & 100.00 & 1.00 \\
\hline 2 & $1 \mathrm{~L}$ & 1 & 100 & 100.00 & 1.00 \\
\hline 3 & $6 \mathrm{~S}$ & 8 & 90 & 99.91 & 0.86 \\
\hline 4 & $7 \mathrm{P}$ & 6 & 73 & 99.93 & 0.80 \\
\hline 5 & $2 \mathrm{~L}$ & 2 & 100 & 100.00 & 1.00 \\
\hline 6 & $6 \mathrm{~S}$ & 8 & 63 & 99.81 & 0.59 \\
\hline Avg & & & $\mathbf{8 7 . 5 8}$ & $\mathbf{9 9 . 9 4}$ & $\mathbf{0 . 8 7 5}$ \\
\hline Stdev & & $\mathbf{1 6 . 1 8}$ & $\mathbf{0 . 0 8}$ & $\mathbf{0 . 1 6}$ \\
\hline
\end{tabular}

\subsubsection{Objective Multisensor Comparison}

The second objective was to directly compare the pressure sensor array to the relevant PSG sensors. Table 5.3 presents multiple detection scenarios compared to the expert interpretation of the PSG signal. The number of TP CA detection is indicated in brackets along with the number of FPs followed by the position in which the FP occurred. 
Table 5.3 CA detection results for multiple sensor scenarios showing true positives (TP) and false positives (FP). Notation: \#TP (\#FP)

\begin{tabular}{|c|c|c|c|c|c|c|c|}
\hline$P t \#$ & $\begin{array}{l}\text { Scoring } \\
(\# C A)\end{array}$ & Respi. only & $\begin{array}{l}\text { Respi. + } \\
\text { Airflow }\end{array}$ & $\begin{array}{l}\text { Respi. } \\
+\mathrm{SpO}_{2}\end{array}$ & $\begin{array}{l}\text { Array } \\
\text { only }\end{array}$ & $\begin{array}{l}\text { Array + } \\
\text { Airflow }\end{array}$ & $\begin{array}{l}\text { Array } \\
+\mathrm{SpO}_{2}\end{array}$ \\
\hline 1 & 1 & 1 & 1 & 0 & 1 & 1 & 0 \\
\hline 2 & $1 \mathrm{~L}$ & $1(1)$ & $(1)$ & 1 & 1 & 1 & 0 \\
\hline 3 & $6 S$ & $6(2)$ & $6(2)$ & 5 & $6(2)$ & $6(2)$ & $5(1)$ \\
\hline 4 & $7 \mathrm{P}$ & $3(1)$ & $3(1)$ & 2 & $5(1)$ & $5(1)$ & $4(1)$ \\
\hline 5 & $2 \mathrm{~L}$ & $2(13)$ & $2(1)$ & $0(4)$ & 2 & 2 & 1 \\
\hline 6 & $6 \mathrm{~S}$ & $4(4)$ & $3(3)$ & 1 & $4(4)$ & $4(4)$ & 0 \\
\hline
\end{tabular}

The CA detections obtained with the Array can be compared to those obtained with and without supplemental information from the airflow sensor (or thermistor when the airflow sensor data was unavailable). The 'Respi+SpO${ }_{2}$ ' and 'Array $+\mathrm{SpO}_{2}$ ' columns indicate how well the respibands and pressure array detect CAs which caused an oxygen desaturation in the patient. Table 5.4 presents value of Cohen's kappa which was calculated for the signal combinations presented in Table 5.3.

Table 5.4 Cohen's kappa coefficient for CA classification results for multiple sensor scenarios compared to PSG scoring

\begin{tabular}{|lllllll|}
\hline Pt\# & $\begin{array}{l}\text { Respi. } \\
\text { only }\end{array}$ & $\begin{array}{l}\text { Respi. }+ \\
\text { Airflow }\end{array}$ & $\begin{array}{l}\text { Respi. } \\
+\mathrm{SpO}_{2}\end{array}$ & $\begin{array}{l}\text { Array } \\
\text { Only }\end{array}$ & $\begin{array}{l}\text { Array } \\
\text { Airflow }\end{array}$ & $\begin{array}{l}\text { Array } \\
+\mathrm{Sp0}_{2}\end{array}$ \\
\hline $\mathbf{1}$ & 1.00 & 1.00 & 0.00 & 1.00 & 1.00 & 0.00 \\
\hline $\mathbf{2}$ & 0.40 & 0.40 & 0.67 & 1.00 & 1.00 & 1.00 \\
\hline $\mathbf{3}$ & 0.78 & 0.78 & 0.89 & 0.86 & 0.86 & 0.84 \\
\hline $\mathbf{4}$ & 0.59 & 0.59 & 0.50 & 0.80 & 0.80 & 0.67 \\
\hline $\mathbf{5}$ & 0.20 & 0.57 & 0.40 & 1.00 & 1.00 & 0.67 \\
\hline $\mathbf{6}$ & 0.47 & 0.50 & 0.20 & 0.59 & 0.50 & 0.22 \\
\hline Avg. & $\mathbf{0 . 5 7}$ & $\mathbf{0 . 6 4}$ & $\mathbf{0 . 4 4}$ & $\mathbf{0 . 8 7 5}$ & $\mathbf{0 . 8 6}$ & $\mathbf{0 . 5 7}$ \\
\hline Stdev. & $\mathbf{0 . 2 8}$ & $\mathbf{0 . 2 2}$ & $\mathbf{0 . 3 2}$ & $\mathbf{0 . 1 6}$ & $\mathbf{0 . 2 0}$ & $\mathbf{0 . 3 8}$ \\
\hline
\end{tabular}

* data analyzed in $30 \mathrm{~s}$ epochs

To quantify how much the detection obtained with the array and other signal combinations resemble the scoring, the interrater reliability was evaluated using the intraclass correlation coefficient on the class judgments. The two-way mixed model as 
directed by Shrout and Fleiss [80] in IBM SPSS Statistics Version 19 was used to calculate the ICC. Table 5.5 shows the ICC coefficient calculated between the expert scoring and the output obtained by using the signal combination listed at the top of each column.

Table 5.5 ICC for CA classification for multiple sensor scenarios compared to PSG scoring

\begin{tabular}{|lllllll|}
\hline Pt\# & $\begin{array}{l}\text { Respi. } \\
\text { only }\end{array}$ & $\begin{array}{l}\text { Respi. }+ \\
\text { Airflow }\end{array}$ & $\begin{array}{l}\text { Respi. } \\
+\mathrm{SpO}_{2}\end{array}$ & $\begin{array}{l}\text { Array } \\
\text { Only }\end{array}$ & $\begin{array}{l}\text { Array } \\
\text { Airflow }\end{array}$ & $\begin{array}{l}\text { Array } \\
+\mathrm{Sp0}_{2}\end{array}$ \\
\hline $\mathbf{1}$ & 1.00 & 1.00 & 0.00 & 1.00 & 1.00 & 0.00 \\
\hline $\mathbf{2}$ & 0.57 & 0.57 & 0.80 & 1.00 & 0.00 & 1.00 \\
\hline $\mathbf{3}$ & 0.88 & 0.88 & 0.94 & 0.92 & 0.92 & 0.91 \\
\hline $\mathbf{4}$ & 0.58 & 0.61 & 0.48 & 0.89 & 0.89 & 0.80 \\
\hline $\mathbf{5}$ & 0.33 & 0.73 & 0.57 & 1.00 & 1.00 & 0.80 \\
\hline $\mathbf{6}$ & 0.64 & 0.67 & 0.33 & 0.74 & 0.65 & 0.33 \\
\hline Avg. & $\mathbf{0 . 6 7}$ & $\mathbf{0 . 7 4}$ & $\mathbf{0 . 5 2}$ & $\mathbf{0 . 9 3}$ & $\mathbf{0 . 7 4}$ & $\mathbf{0 . 6 4}$ \\
\hline Stdev. & $\mathbf{0 . 2 0}$ & $\mathbf{0 . 1 2}$ & $\mathbf{0 . 2 5}$ & $\mathbf{0 . 1 0}$ & $\mathbf{0 . 3 9}$ & $\mathbf{0 . 3 9}$ \\
\hline \multicolumn{7}{|c}{ data analyzed in $30 \mathrm{~s}$ epochs } \\
\end{tabular}

\subsubsection{Effect of Desaturation Criteria on CA Scoring and Classification}

The $\mathrm{SpO}_{2}$ sensor reported the oxygenation percentage in increments of $1 \%$. The desaturation criterion was therefore set to the smallest possible value, $1 \%$, to retain the largest number of events. A function was created to identify desaturations during the scored CA with the addition of a $1 \mathrm{~s}$ buffer on either side. Table 5.6 shows how the processed array and respiband signals are affected by the inclusion of a desaturation criterion on the scoring which is the third objective of the analysis. The criterion was first applied to the scoring. The remaining number of CAs present in each patient's file is presented in the "Modified Scoring" column of Table 5.6. Some files, such as Pt\# 1 no longer contained any CAs. The Respi. and Array columns report the total number of positive detections while the remaining three columns report the classification parameters of the Array sensor. 
Table 5.6 Effect of including a desaturation criterion on CA Classification, Measured with Cohen's Kappa

\begin{tabular}{|lllllll|}
\hline Pt\# & $\begin{array}{l}\text { Modified } \\
\text { Scoring }\end{array}$ & $\begin{array}{l}\text { Respi. } \\
+\mathbf{S p 0}_{2}\end{array}$ & $\begin{array}{l}\text { Array } \\
+\mathbf{S p 0}_{2}\end{array}$ & $\begin{array}{l}\text { Array } \\
\text { Sens. }\end{array}$ & $\begin{array}{l}\text { Array } \\
\text { Spe. }\end{array}$ & $\begin{array}{l}\text { Array } \\
\mathbf{K}\end{array}$ \\
\hline $\mathbf{1}$ & $\mathbf{0}$ & 1 & 1 & 100.00 & 100.00 & 1.00 \\
\hline $\mathbf{2}$ & 1 & 5 & 8 & 100.00 & 99.10 & 0.86 \\
\hline 3 & 6 & 6 & 72.73 & 99.93 & 0.80 \\
\hline $\mathbf{4}$ & 7 & 4 & 6 & 100.00 & 99.95 & 0.67 \\
\hline $\mathbf{5}$ & 1 & 4 & 2 & 100.00 & 100.00 & 1.00 \\
\hline $\mathbf{6}$ & 1 & 1 & 1 & $\mathbf{9 4 . 5 5}$ & $\mathbf{9 9 . 8 0}$ & $\mathbf{0 . 8 7}$ \\
\hline Avg. & & & $\mathbf{1 2 . 2 0}$ & $\mathbf{0 . 3 9}$ & $\mathbf{0 . 1 4}$ \\
\hline Stdev. & & & & &
\end{tabular}

No CAs occur along with a $1 \%$ desaturation criterion

\subsubsection{CA Detection Example}

This section shows two examples of PSG (pressure sensor, Chest and abdominal respiband, airflow thermistor (TH Flow)) and Array signal pairs. Each figure shows data for two consecutive 30 s epochs. On the next page, Figure 5.10 shows the apnea model applied to the pressure sensor variance signal while Figure 5.11 shows the corresponding PSG signal. The CA in this case is easily distinguished from the neighboring data which contain clear breathing patterns in all sensors. Three lines are shown on Figure 5.10 the summed variance signal from the pressure sensor array (Variance), the output of the proposed approach (CA Algorithm) and the sleep laboratory expert interpretation (Scoring).

Figure 5.12 shows the fused signal variance along with the predicted apnea and the scored apnea for $60 \mathrm{~s}$ of data. The summed variance signal from the pressure sensor array (Variance), the output of the proposed approach (CA Algorithm) and the sleep laboratory expert interpretation (Scoring) are again shown on Figure 5.12. Figure 5.13 shows the PSG signals for 60 s of data. It is possible to see two distinct areas of stillness for the 
respibands. The first area is not scored as a CA while the second is. This illustrates some of the challenges in detecting CAs both for the expert interpreters and for algorithms relying on those interpretations.

\subsection{Discussion}

Data from patients with a BMI $>35 \mathrm{~kg} / \mathrm{m}^{2}$ was not classified as accurately as those with a BMI $<35$. These patients tended to have a very high number of hypopneas, which were often falsely detected as CAs. Mack et al. [42] also reported less reliable estimates of apnea with increasing AHI.

As such, the classifier presented in this chapter must take into account the weight and height of the bed occupant. This easily available and static data provides one contextual limitation of the pressure sensor array. While CAs may not be detected, it is quite possible that large movements such as rollovers and some breathing analysis may still be performed successfully. Table 5.2 showed that the pressure sensor array provided a very good detection of the CAs in the PSG data for the six patients studied whose BMI was appropriate for the sensor. For the valid patients, the algorithm classified the CA events with an average sensitivity of $87.58 \%$, specificity of $99.94 \%$ and Cohen's kappa value of 0.875 when the array was compared directly to the scoring. 


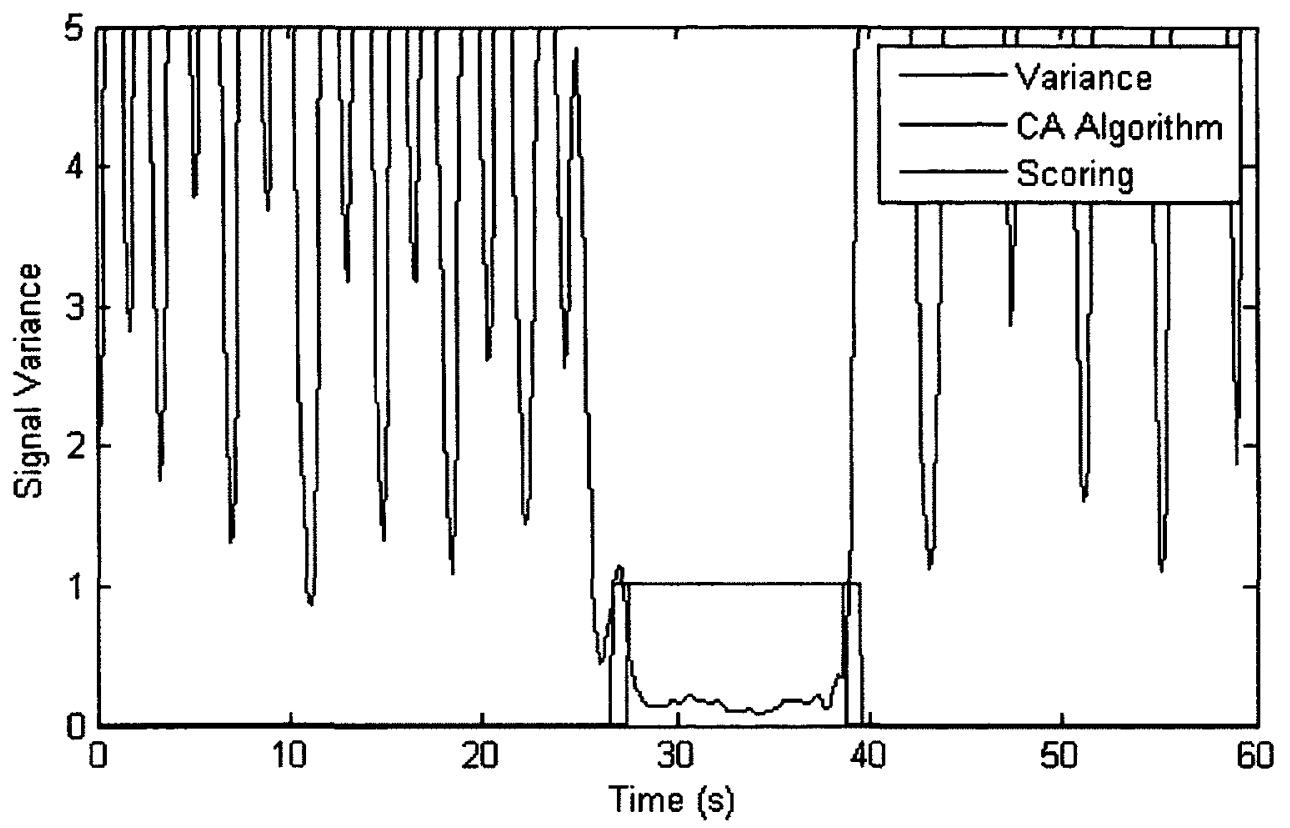

Figure 5.10 Processed sensor array data showing correct identification of CA event (from approx. $28 \mathrm{~s}$ to $40 \mathrm{~s}$ )

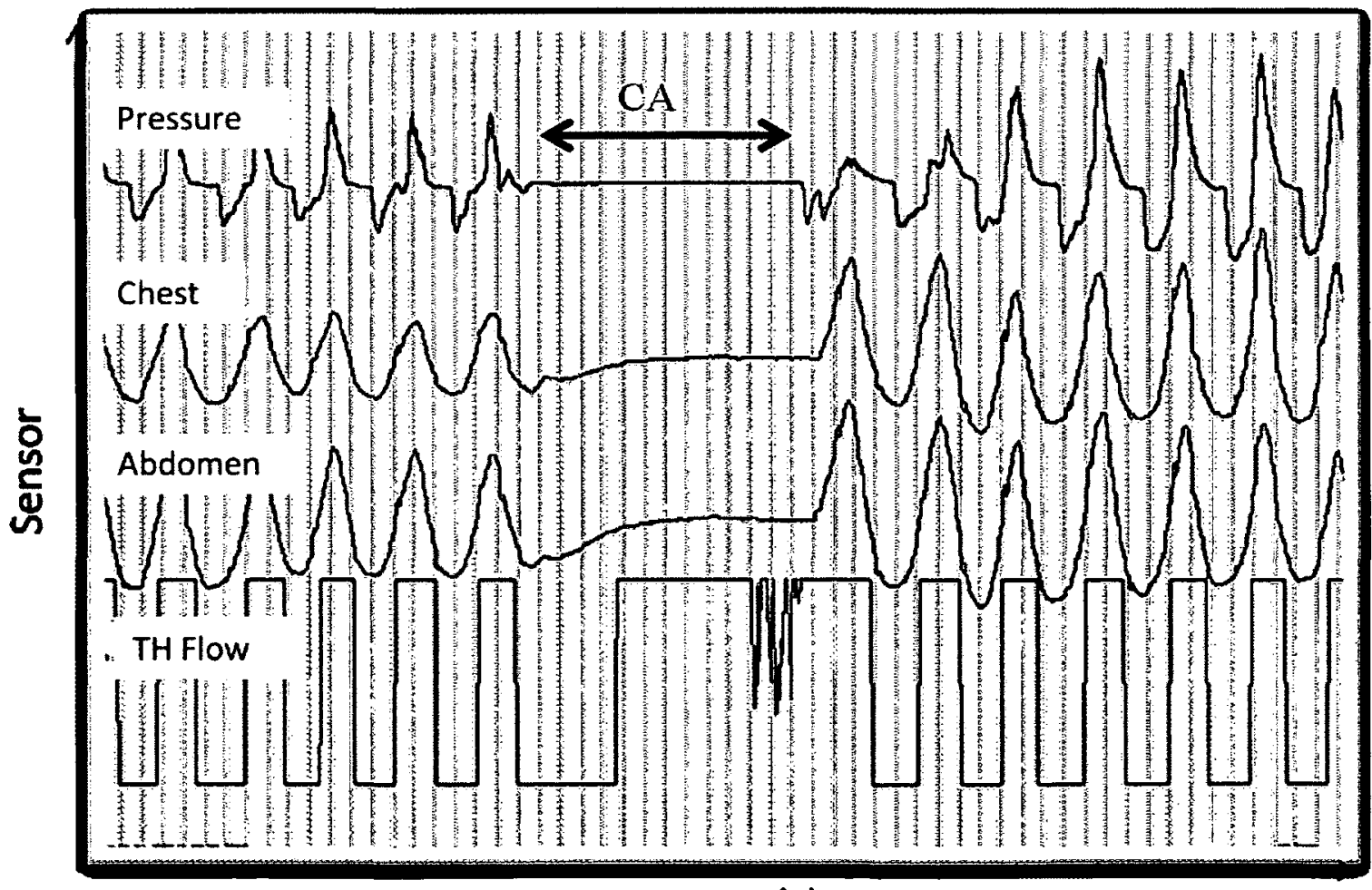

Time (s)

Figure 5.11 PSG signals for oronasal air pressure, and chest and abdomen respiration bands 


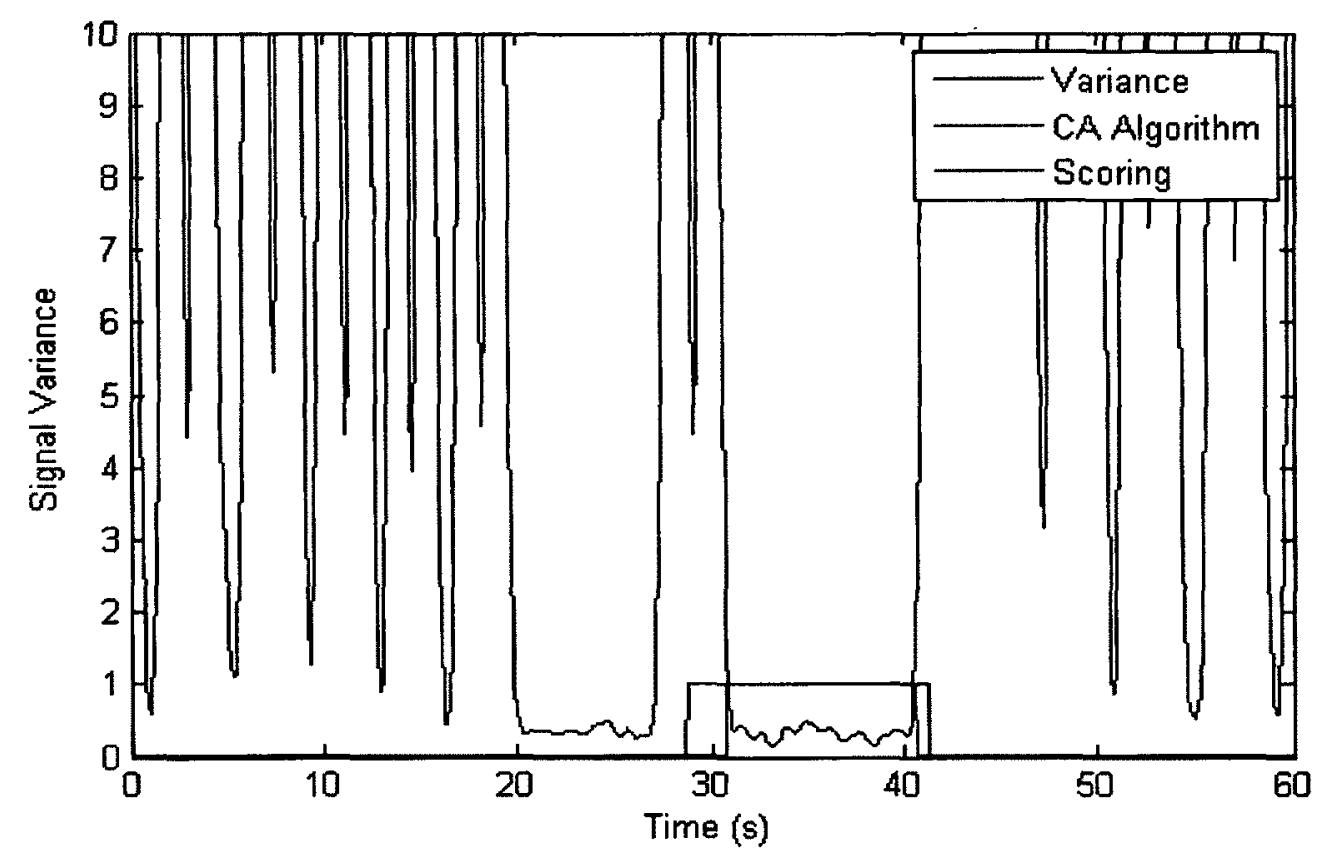

Figure 5.12 Correct classification of one apnea

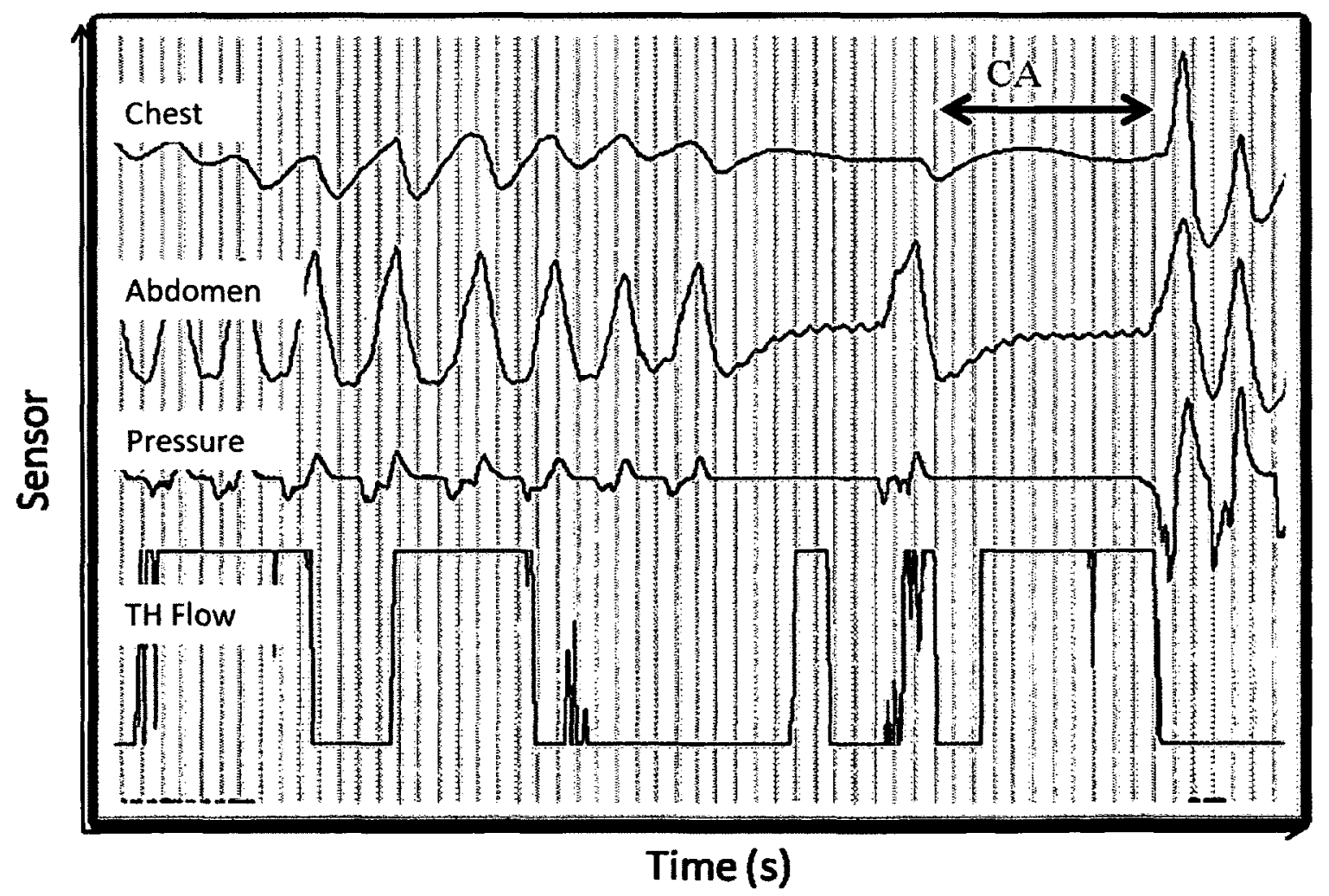

Figure 5.13 PSG signals for one CA 
These results are an improvement over results reported by Beattie et al. [49] who used four PSG patients. The average interscorer reliability between the array and the scoring of 0.875 compares well with Jensen et al. who found $\kappa=0.87$ in 18 control patients, suggesting that the error between the scoring and the pressure sensor array is no greater than the error that would occur between two human expert scorers [39]. The present results also compare well with Shin et al. who obtained a sensitivity of $88 \%$ using an air mattress [79].

Table 5.6 showed that the inclusion of a desaturation criterion on the CA requirements in the PSG data improves classifier performance. A similar effect was reported by Morillo et al. amongst others, suggesting that in this measure, the pressure sensor array data also behaves in a similar fashion to the respibands [58].

This chapter met objective 1 in section 5.4 .3 by showing that for every patient whose BMI was appropriate for the sensor there is a set of parameters for the model that correctly detects scored apneas with good classification accuracy.

To meet objective 2, section 5.4.4 used Cohen's kappa to measure the agreement between two raters beyond what would occur by chance and used the ICC, a form of reliability testing, to evaluate the sensor combinations. The sensor array's ability to detect CAs in this validation dataset was presented. The ICCs of Respi+Airflow and Array+Airflow columns were equal. This suggests that the array provides equivalent discrimination to the respibands when used in conjunction with the airflow sensor as is the case during a PSG interpretation.

To meet objective 3 , section 5.4 .5 showed that the array used in the context of apneas with a desaturation was capable of detecting them very accurately. CAs do not have a 
$\mathrm{SpO}_{2}$ desaturation requirement as per AASM guidelines, however hypopneas do. The results of section 5.4.5 suggest that the array could also be used to detect some hypopneas.

The sensor arrays remained in place, below the mattress at the sleep laboratory for many months. During that time they did not require any adjustment to their position. The loading levels of the sensors was visually assessed during the biweekly data collections and the number of sensors activated, but not saturated by the weight of the mattress remained stable, and did not vary throughout the experiments. This suggests that although sensor values may drift over a short period of time as reported in Chapter 3, they do not drift until saturation.

\subsection{Conclusions}

The pressure sensitive array was successful at detecting CAs in PSG data obtained from patients at the West Ottawa Sleep Center who consented to the study. The pressure sensor array successfully detected the majority of CAs scored by the polysomnographers. The inclusion of a desaturation criterion on CA scoring affected the pressure sensor array classification similarly to the respiband classification as reported in the literature. Context-awareness allows the system to not produce apnea detections for patients above the limit BMI with this algorithm. This clinical data contains some of the same features noticed in the controlled experiment data, notably a difference in the signals captured depending on lying position. Position changes occur with a rollover, which the next chapter presents. 


\section{Chapter 6: Controlled Experiment for Rollover Classification}

\subsection{Introduction}

As introduced in Chapter 2, in addition to being associated with specific sleep states, rollovers indicate position changes. Positional sleeping has an effect on the severity of multiple breathing disorders including sleep apnea. This chapter presents an algorithm for rollover classification using features extracted from the pressure data ${ }^{8}$. Section 6.2 describes the controlled experiment and Section 6.3 presents the proposed approach. The results and discussion are in Section 6.4 while conclusions are presented in Section 6.5 .

\subsection{Multiple Sensor Positions - Controlled Experiment}

The pressure sensor array was installed between a standard hospital 4-inch mattress and a Stryker Medical $190 \mathrm{~cm}$ by $86 \mathrm{~cm}$ metal adjustable bed frame remaining in a flat position throughout the experiment. One standard hospital pillow was placed at the head of the

\footnotetext{
${ }^{8}$ A significant portion of the contents in this chapter is published in [8].
} 
bed. The bed sections measure approximately $79,23,36$ and $51 \mathrm{~cm}$. Tests were conducted with the sensor in five positions as shown in Figure 6.1. A healthy volunteer performed a series of large movements (bed entry/exists, rollovers, rocking, sleep startles, posture shifts) and small movements (arm/leg twitches, gasps) with the sensor in each position. An observer noted the times of movement onset and position changes to allow for manual annotation of the data. In total 177 movements were recorded: 60 rollovers and 117 other movements. They are shown in Table 6.1. The objectives of this controlled experiment are: 1) create decision trees for rollover classification, and 2) rank feature importance.
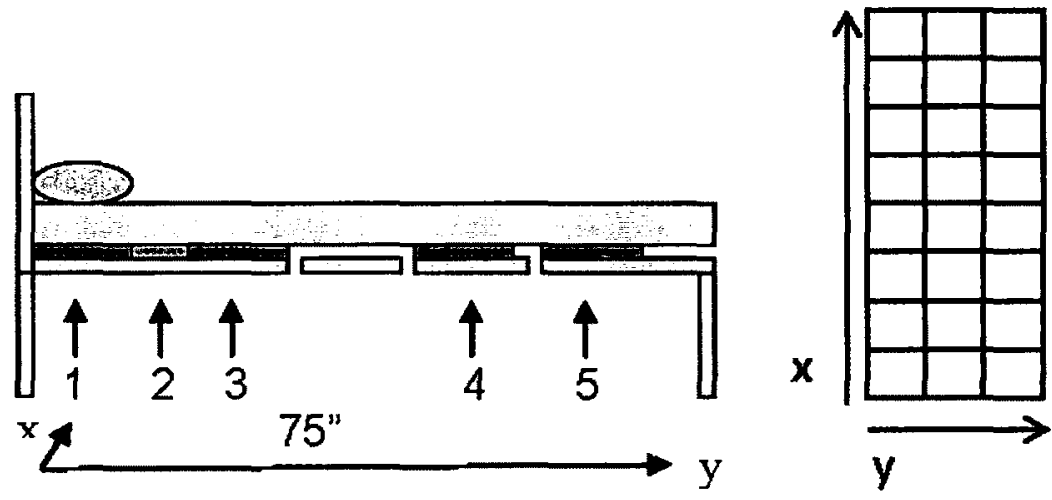

Figure 6.1 Diagram of hospital bed showing sensor placement and grid layout of sensor elements

Table 6.1 Number and type of movements for each pressure sensor position

\begin{tabular}{|c|c|c|c|c|c|}
\hline Movement & Pos. 1 & Pos. 2 & Pos. 3 & Pos. 4 & Pos. 5 \\
\hline Entry & 8 & 11 & 5 & 7 & 4 \\
\hline Exit & 7 & 10 & 5 & 7 & 3 \\
\hline Rollover & 15 & 14 & 10 & 10 & 11 \\
\hline $\operatorname{Leg}(s)$ & 0 & 0 & 0 & 6 & 1 \\
\hline $\operatorname{Arm}(\mathbf{s})$ & 0 & 1 & 5 & 0 & 0 \\
\hline Body movement & 0 & 8 & 12 & 5 & 12 \\
\hline Total & 30 & 44 & 37 & 35 & 31 \\
\hline
\end{tabular}




\subsection{Classification Approach}

The beginning and end of each movement was identified manually and the features for each movement were extracted and used in a decision tree. MATLAB's (R2009a) decision tree classes were selected to build a classifier because their intuitive interpretation is appropriate for exploratory work and pruning can identify salient features [81]. Pruning was performed by finding the smallest tree with an error within one standard deviation of the best classification tree's resubstitution error. This provides computational savings. Pruning balances "predictive accuracy and model complexity for applications where model interpretation is still desired" [81]. The features extracted from the sensor data for each annotated movement are listed in Table 6.2. The five are salient features in rollovers.

Table 6.2 Extracted features for each movement

\begin{tabular}{|ll|}
\hline Parameter & \multicolumn{1}{c|}{ Description } \\
\hline Amplitude & Amplitude of the CoG $x$ signal \\
\hline Duration & Duration of fluctuations \\
\hline \# Excurs. & Number of excursions in CoG $x$ signal \\
\hline $\boldsymbol{\Delta}$ & $\begin{array}{l}\text { Difference between final and initial } \\
\text { CoG } x \text { position }\end{array}$ \\
\hline Mvar & $\begin{array}{l}\text { Average value of moving variance } \\
\text { calculated with } 1 \text { s window }\end{array}$ \\
\hline
\end{tabular}

A measure of the center of gravity in the $\mathrm{x}$ direction $(\mathrm{CoG} x)$ was used to estimate the person's placement on the sensor array. First the total pressure on each of the eight rows, $\mathrm{Wr}$, was calculated with eq. (6.1) where $\mathrm{p}_{\mathrm{i}, \mathrm{j}}$ is the pressure received by the sensor on the $i^{\text {th }}$ row and $j^{\text {th }}$ column as indicated in Figure 6.1. The most heavily weighted row was then used to approximate a CoGx signal. Each sensor element was weighted equally. 
$W r_{i}=\sum_{j=1}^{3} p_{i, j}$

Figure 6.2 shows the pressure array output for four position changes and below, the CoGx signal for the same data. To meet objective 1), a decision tree was generated using the data from each sensor position with a stratified 10-fold cross-validation. Then, movements from all sensor position files were combined to build one general tree. For objective 2), linear discrimination analysis measured the relative importance of features from the single-position decision trees.

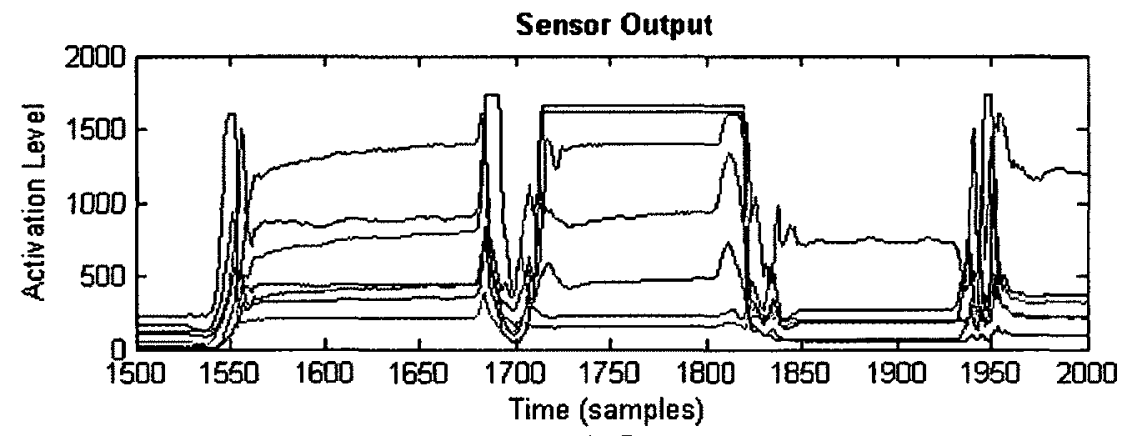

$\operatorname{CoG} x$

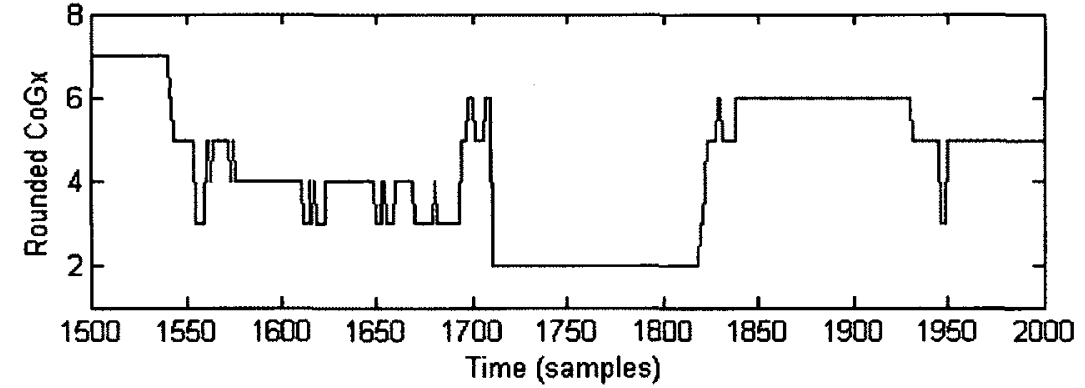

Figure 6.2 Sensor data during four rollovers (top) and approximated center of gravity signal (bottom)

\subsection{Results and Discussion}

Table 6.3 shows the intrapositional classification results for each pruned tree built with single position sensor data. These results indicate that the ideal sensor position for 
rollover classification is position 4 (placed near the thigh), though position 2 and 3 (near the chest and abdomen) maintain good classification.

Table 6.3 Intrapositional classification results for rollover detection (pruned tree)

\begin{tabular}{|l|l|l|l|l|l|l|l|}
\hline \multicolumn{2}{|c|}{ Pos. 1} & Pos.2 & Pos.3 & \multicolumn{1}{l}{ Pos. 4 } & Pos. 5 & \multicolumn{1}{l|}{ Avg \pm Stdev } & General Tree \\
\hline Sens. & 73.3 & 100 & 100 & 100 & 100 & $94.7 \pm 11.9$ & $\mathbf{8 1 . 7}$ \\
\hline Spe. & 93.3 & 96.7 & 96.2 & 100 & 75 & $92.3 \pm 9.9$ & $\mathbf{1 0 0}$ \\
\hline \hline MCC & $\mathbf{0 . 7}$ & $\mathbf{1 . 0}$ & $\mathbf{0 . 9}$ & $\mathbf{1}$ & $\mathbf{0 . 7}$ & $\mathbf{0 . 8} \pm \mathbf{0 . 2}$ & $\mathbf{0 . 9}$ \\
\hline
\end{tabular}

Data from positions 1 and 5 may be better suited for head and leg movement classification respectively. In controlled experiments $A, B$ and $C$, the sensor was positioned length-wise along the bed and covered positions 2 and 3. Analysis from a fully sensorized bed could focus on sensors from these regions. This is further supported by observing that the general tree provided reduced discrimination ability compared to individual positions 2,3 , and 4 as measured by MCC and percentage of movements in correct class. As such it may improve classification results to narrow the focus onto certain regions of a fully sensorized bed. The results of Table 6.4, ranking the relative importance of features using linear discriminant analysis, suggest that feature importance also changes with sensor position as movements are transferred to pressure sensors differently along the length of the bed. When the sensor is closer to the head or foot of the bed, other features may be required to provide the same classification rate as in centered positions. The type of movement most likely to be misclassified was side-to-side rocking, a movement which resembles a failed rollover attempt. The features related to the repetitive nature of this movement can be studied and added to the features list to increase specificity in rollover detection. The duration of rollovers in this study was 5.4 $\pm 4.4 \mathrm{~s}$, though it may be significantly longer for older adults who may require several attempts to change position. An increased number of attempts are expected to affect the 
number of fluctuations in the CoGx signal. The \# Excurs. feature captures some of the movement duration information, however including actual movement durations may provide additional information to the classifier.

Table 6.4 Relative feature importance for rollover detection for each positional tree

\begin{tabular}{|l|l|l|l|l|}
\hline Pos. 1 & Pos. 2 & Pos. 3 & Pos. 4 & Pos. 5 \\
\hline$\Delta$ & $\Delta$ & $\Delta$ & \# Excurs. & Duration \\
\hline Duration & \# Excurs. & Amplitude & $\Delta$ & $\Delta$ \\
\hline \# Excurs. & Mvar & \# Excurs. & Duration & \# Excurs. \\
\hline Amplitude & Amplitude & Mvar & Amplitude & Mvar \\
\hline Mvar & Duration & Duration & Mvar & Amplitude \\
\hline
\end{tabular}

The generalized pruned tree correctly classified $81.7 \%$ of rollovers (49 out of 60 ) and $100 \%$ of other movements (117 out of 117$)$. The proportion of movements in the correct class was $93.8 \%$, which compares with other measures reported in the literature. Miwa et al. [28] detected rollovers in patients wearing a SenseWear Pro2 Armband. Thresholding the maximum and mean acceleration (in $\mathrm{x}$ and $\mathrm{y}$-axis) identified posture differences "when the actigraph detected 'sleep" and $82.4 \%$ (400 out of 475 ) of rollovers were detected. Walsh et al. [82] collected data simultaneously from a pressure sensor array and wrist-worn actigraph. Thresholds on the pressure sensor's value for standard deviation and squared difference were used to map the movements detected by actigraphy to the unobtrusive sensor with $69.6 \%$ sensitivity and $89.6 \%$ specificity $(86.7 \%$ correct classification rate). Compliance problems with the use of actigraphy were reported for two of the ten study volunteers because of device positioning and device obtrusiveness. Foubert [50] used a full length sensor array to recognize six static postures and the lie to sit transition. Rollover classification as provided in this chapter acts as a preprocessing step to posture estimation by recognizing posture shifts to enable "meaningful 
segmentation of pressure signals as a preprocessing step before applying posture-specific algorithms".

\subsection{Conclusions}

The unobtrusive pressure sensor array can be part of a long-term monitoring strategy in smart homes and used to detect specific movements in bed such as rollovers and gain knowledge about sleep patterns. Studying the effect of sensor placement on classification of movements and feature importance provided useful information for outfitting smart homes where it is desirable to optimize the number of sensors (minimizing cost while maintaining detection properties). Since the general tree did not perform as well as individual trees, it may not be necessary to fully cover the bed in sensor arrays for optimal rollover detection. Rollovers are an important event in normal and fragment sleep as they mark movement and position changes. The use of the pressure sensor array offers advantages compared to actigraphy and PSG in terms of cost and obtrusiveness and can be used to provide context for other algorithms. 


\section{Chapter 7: \\ Analysis using Delay Profile}

\subsection{Introduction}

In an array multiple sensors can have common information, and if combined properly can create a higher amplitude signal, improve the SNR, and result in a signal that is more easily analyzed. This chapter presents an algorithm to measure torso movement and create a delay profile for the sensor array ${ }^{9}$. Section 7.2 describes the proposed approach and the results are presented in Section 7.3. The discussion takes place in Section 7.4 and the chapter concludes with Section 7.5

\subsection{Proposed Approach}

Eight healthy participants (20-35 yrs old) volunteered to lie on a mattress while wearing respibands. Two pressure sensor arrays were placed beneath the single mattress, $10 \mathrm{~cm}$ apart, as shown in Figure 7.1. In total, 48 pressure sensors are located under the mattress, beneath the person's torso, approximately every $10 \mathrm{~cm}$ in an $8 \times 6$ framework. Participants were asked to produce normal, deep and shallow breaths, and movements while in four different positions (left and right side, supine and prone). Ethical approval was obtained from Carleton University, and the participants signed informed consent forms. Respiband

\footnotetext{
${ }^{9}$ A significant portion of this chapter is published in [9] and [10], and additional work related to signal combining during movement is published in [11].
} 
signals were time stamped and down sampled to $10 \mathrm{~Hz}$ to match the pressure array signals.

Figure 7.2 shows the block diagram for the sensor alignment and combining approach. The block diagram shows how the participant data is preprocessed in section 7.2.2. Section 7.2.3 addresses the polarity of the sensors and section 7.2.4 calculates the delay values. Valid sensors are determined in section 7.2.5.

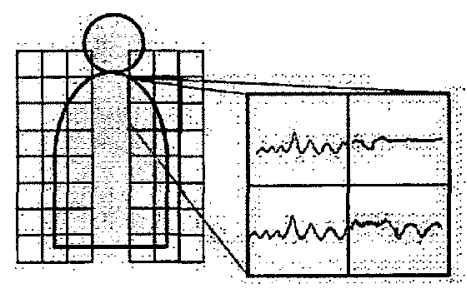

Figure 7.1 Sensor positioning beneath volunteer and change in pressure signal shown for four sensors during normal breathing

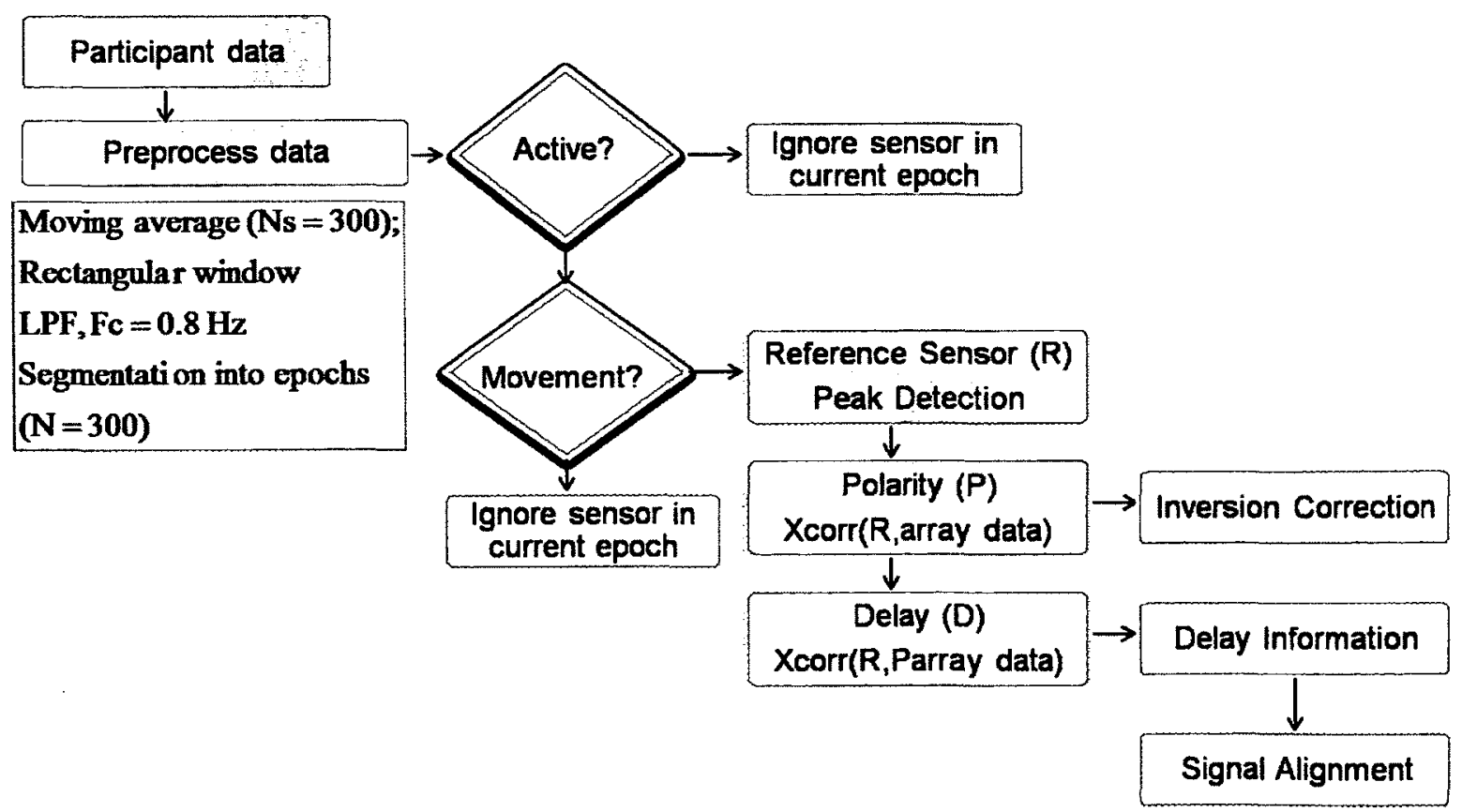

Figure 7.2 Block diagram showing steps of sensor alignment, and delay correction 


\subsubsection{Origin of Delays and Inversions}

Two array attributes require attention before the data fusion. They are i) signal inversion and ii) signal delay. The pressure sensor array captures breathing motion generated along the torso and shoulders of the participant. These signals can have different polarities due to their position relative to the participant's torso which may apply pressure to the elements of the array at slightly different times. However there is another source of inverted signals; pressure applied to an area of the rigid plastic covering the array can cause the area next to it to bend upward, thereby creating an artifact of pressure reduction on an adjacent sensor. Figure 7.3 illustrates this effect. The top signal has greater amplitude than the second signal, however they have opposite polarity. Simply adding signals together results in destructive interference - a loss of information.

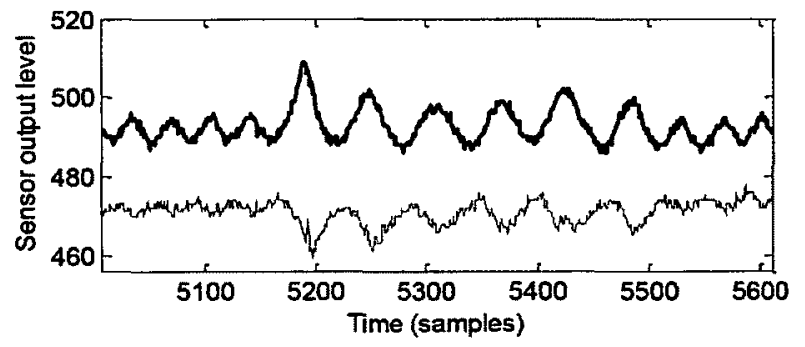

Figure 7.3 Recorded pressure in two adjacent sensors having inverse polarities

The pressure sensor array used in these experiments samples the pressure ten times per second. As such it is possible to determine with precision whether the signals reporting a breathing signal are peak-aligned. The array can capture breathing signals from the shoulders, chest and abdomen. Whether due to the physiology of breathing, or to a particular breathing pattern (e.g.: deep breathing, abdominal breathing), there may be an 
asynchrony between some sensors, resulting in a measurable delay between the peaks of signals measured from the different sensor elements.

\subsubsection{Data Preprocessing}

Fusing the array data into one signal is a multi-step process. The data is first segmented into $30 \mathrm{~s}$ epochs to provide a quantitative analysis of signal strength at each stage in the combining process and to conform to the standard for sleep analysis. Then the mean loading value of the sensor is removed by taking a $30 \mathrm{~s}$ moving average, and each signal is filtered by a low pass filter with a cutoff frequency of $0.8 \mathrm{~Hz}$ in order to focus on the breathing frequencies. Each epoch is then analyzed to determine the signal of largest amplitude.

Since not all signals are centered on zero throughout an epoch, the signal amplitude is approximated by dividing the average peak to valley distance by two. The strongest signal is the reference signal for each epoch. Methods for selecting a reference sensor selection were investigated and published in [11]. These approaches are not presented here as this chapter focuses on the delay profile.

Figure 7.4 shows the filtered output of four selected sensors during three complete breaths. This short period highlights the slight irregularity present in consecutive breaths and the difference between the sensors from different mat locations. The four sensors were selected because they display the properties of interest to this section; strongest (S), delayed (D), advanced (A) and inverted (I). The four sensors detecting these signals are identified in Figure 7.5, where each sensor is represented by a shaded box and the space between the arrays is represented by an empty box. 


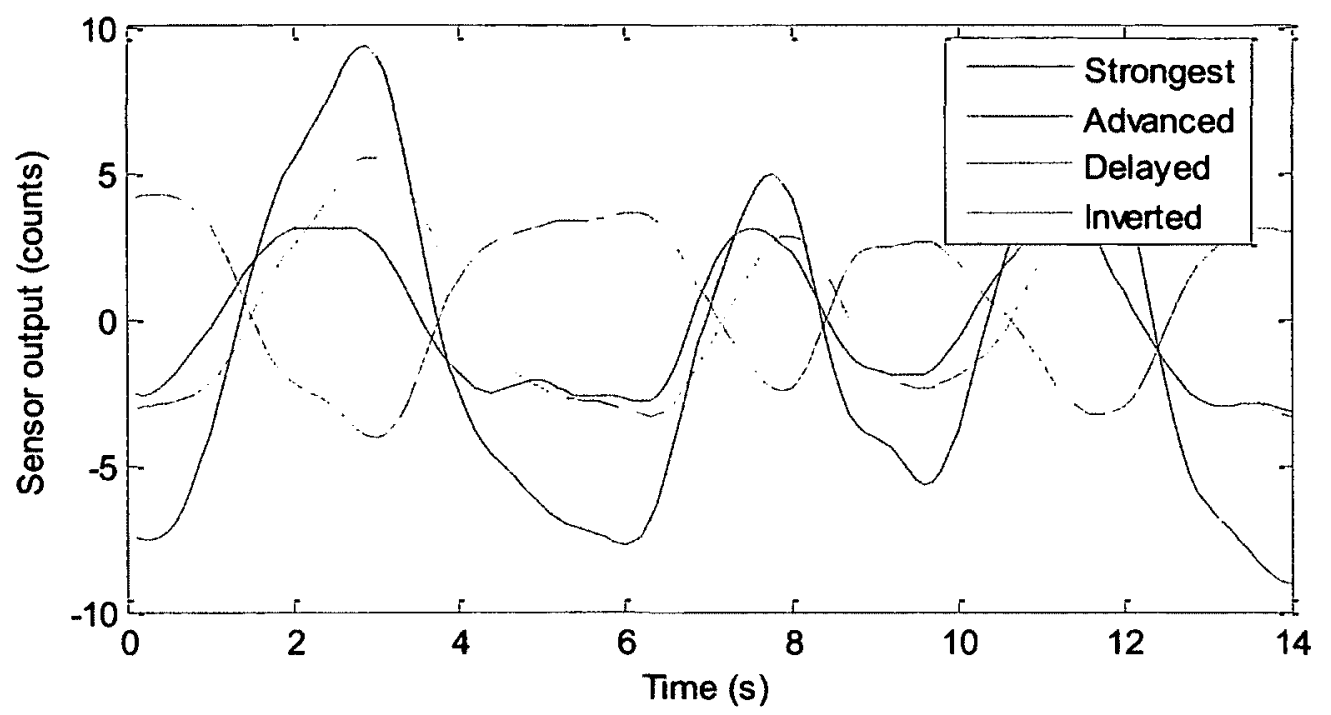

Figure 7.4 Array output showing four sensors with important properties for consideration during alignment and delay mapping

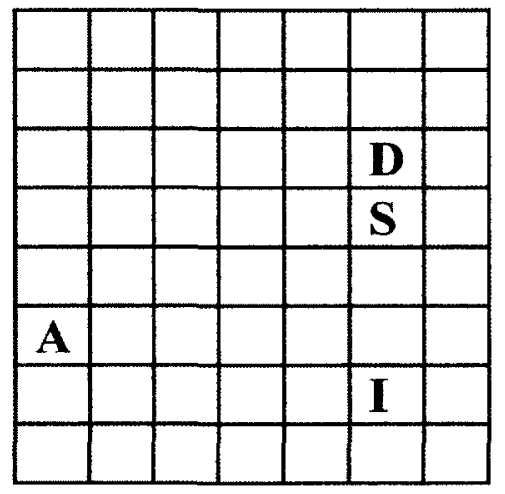

Figure 7.5 Diagram of array instrumentation showing location of sensors with strongest (S), delayed (D), advanced (A) and inverted (I) signals shown in Figure 7.4

\subsubsection{Polarity Identification}

The polarity of each signal compared to the reference signal was determined using their cross-correlation. Each signal is inverted if its cross-correlation value (at delay $\tau=0$ ) with the signal of largest amplitude is stronger with the inverted signal than with the original signal. Figure 7.6 showed a delayed signal, with its crests occurring at roughly the same time as the troughs in the strongest signal. 


\subsubsection{Delay Analysis}

To evaluate the pressure sensor array as a delay measurement system, the delay between the reference and the other signal is calculated using cross-correlation. This reveals information about the synchronization between the sensors. During alignment, the crosscorrelation was constrained to the lag range of $[-10: 10]$ samples $( \pm 1 \mathrm{~s})$ to compel individual breaths to align with the nearest breath in the reference sensor. Lastly, signals are aligned using the delay value that maximizes the cross-correlation and the signals are summed to produce the delay adjusted signal. Figure 7.6 compares the polarity aligned sensors (left) to the time aligned (right) sensors.
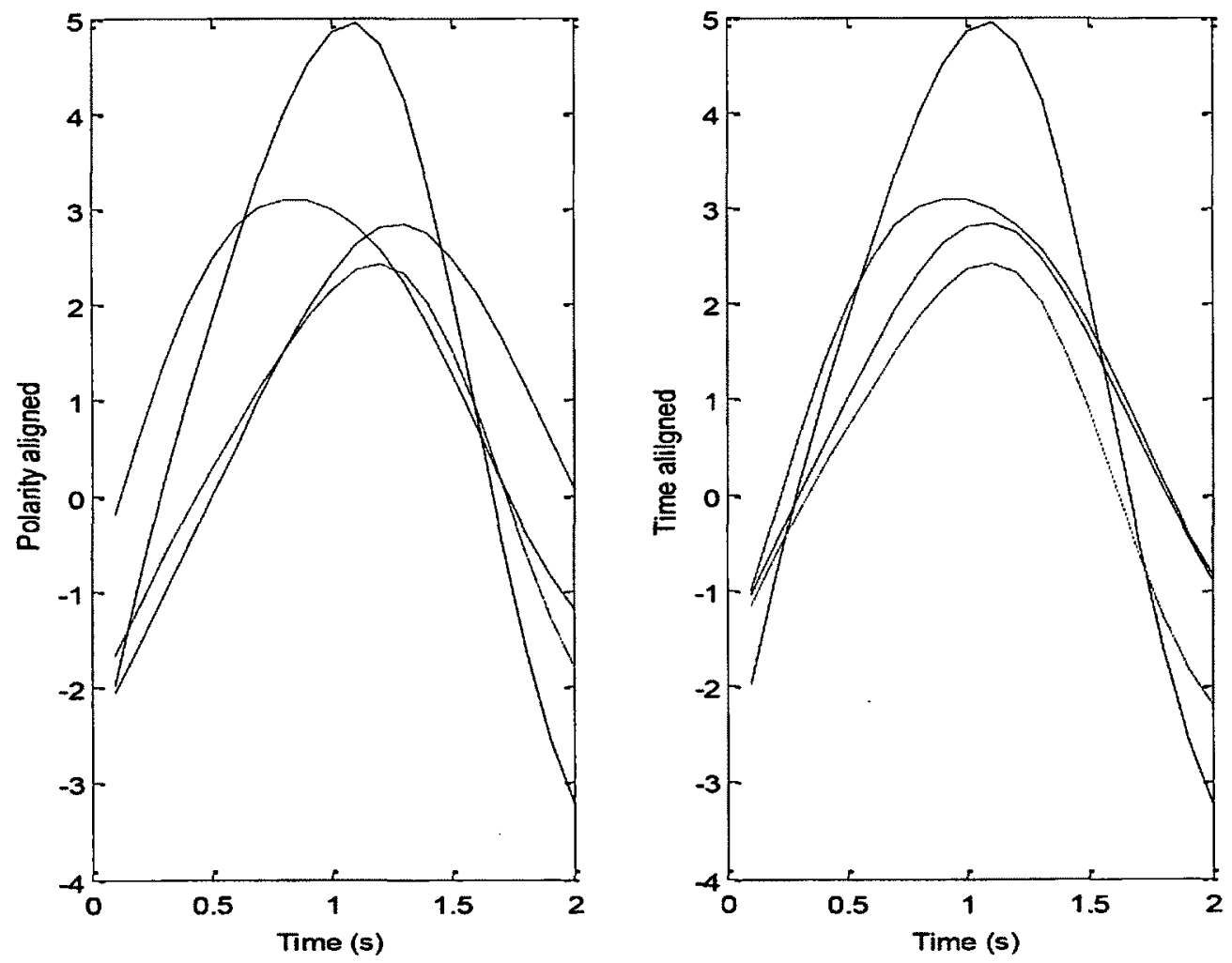

Figure 7.6 Output of four sensors after polarity alignment (left) and after timealignment (right) 


\subsubsection{Selecting Valid Sensors}

A sensor was considered valid if it met two criteria, which introduce upper and lower bounds on the moving variance and moving average:

i) The signal's moving variance was above the threshold indicating apnea for this environment. A threshold on the $1 \mathrm{~s}$ moving variance was determined empirically in [2] for the presence of CA for a participant whose position on the pressure sensor array is unknown (see Section 4.3). The threshold value was modified to 100 , to reflect the effect of the sensor's placement with respect to the mattress on the signal power [1].

ii) No muscular movements were flagged. This experiment uses a previously published movement detection algorithm [52]. To provide further results on movement artifacts in the data, results from the movement detection algorithm were compared to manual movement identification in order to determine the accuracy of the classification of movement corrupted epochs (CEs) with a physical scorer.

Figure 7.7 shows a map of valid sensors in blue. The others (saturated, unloaded or loaded but not meeting conditions i) and ii)) are shown in light purple.

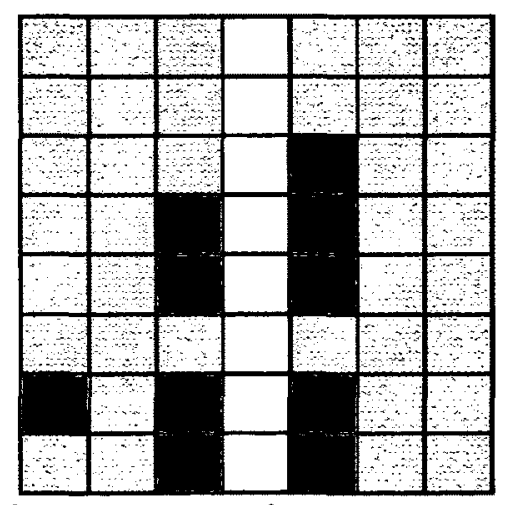

Figure 7.7 Pressure map of sensors showing movement (dark squares) for grid of $8 \times 6$ sensors (gap shown in white) 


\subsection{Results}

\subsubsection{Breathing Signal Availability}

During movements, the proposed method does not produce a signal as CEs are removed. A similar strategy was used by Kuo et al. [70] who described this approach as contextaware processing. Eq. (7.1) describes the corrupted epoch percentage (CE\%), which is the percentage of epochs that have movement reported simultaneously in three or more sensors.

$$
\mathrm{CE} \%=\frac{\# \text { epochs } \text { with movement at } \geq 2 \text { sensors }}{\# \text { epochs in file }} * 100
$$

Table 7.1 compares the automated and manual movement identification. The $\mathrm{CE} \%$ is very similar between methods and the average Sens and Spe values are $71 \%$ and $87 \%$.

Table 7.1 Comparison of automated vs. manual movement detection
\begin{tabular}{|cccccc|} 
Participant\# & Epochs & $\begin{array}{l}\text { Automated } \\
\text { CE\% }\end{array}$ & $\begin{array}{l}\text { Manual } \\
\text { CE\% }\end{array}$ & $\begin{array}{l}\text { Sens } \\
(\%)\end{array}$ & $\begin{array}{c}\text { Spe } \\
(\%)\end{array}$ \\
\hline $\mathbf{1}$ & 282 & 32 & 30 & 83 & 90 \\
\hline $\mathbf{2}$ & 240 & 18 & 18 & 74 & 94 \\
\hline $\mathbf{3}$ & 259 & 28 & 24 & 63 & 83 \\
\hline $\mathbf{4}$ & 255 & 27 & 28 & 69 & 90 \\
\hline $\mathbf{5}$ & 245 & 47 & 32 & 67 & 79 \\
\hline $\mathbf{6}$ & 233 & 31 & 30 & 79 & 90 \\
\hline $\mathbf{7}$ & 231 & 36 & 29 & 82 & 83 \\
\hline $\mathbf{8}$ & 218 & 24 & 30 & 54 & 89 \\
\hline Average & 253 & 29 & 28 & 71 & 87 \\
\hline
\end{tabular}

Table 7.2 presents the number of sensors with breathing signals detected by the two criteria listed, and the average number of sensors affected by movement during CEs. The results show that at least one sensor had breathing information during every epoch (CEs excluded), showing $100 \%$ availability for this data by visual analysis for presence of 
breathing. Each movement affected on average 10 sensors. The $\mathrm{CE} \%$ was high due to the protocol requiring participants to perform a movement every 2 minutes over most of a $2 \mathrm{hr}$ period; a minimum $18 \%$ of epochs had at least three sensor simultaneously affected by movement at some point.

Table 7.2 Number of sensors with breathing or movement

\begin{tabular}{|ccrc|}
\hline Participant\# & $\begin{array}{l}\text { *Average \# of } \\
\text { sensors with } \\
\text { breathing }\end{array}$ & $\begin{array}{l}* \text { Minimum, Maximum] \# } \\
\text { of sensors with breathing }\end{array}$ & $\begin{array}{l}\text { Average \# of sensors } \\
\text { with movement per } \\
\text { corrupted epoch (CE) }\end{array}$ \\
\hline $\mathbf{1}$ & $10 \pm 3$ & {$[4,21]$} & $10 \pm 5$ \\
\hline $\mathbf{2}$ & $8 \pm 2$ & {$[5,16]$} & $12 \pm 7$ \\
\hline $\mathbf{3}$ & $11 \pm 4$ & {$[4,20]$} & $8 \pm 6$ \\
\hline $\mathbf{4}$ & $7 \pm 3$ & {$[2,17]$} & $9 \pm 5$ \\
\hline $\mathbf{5}$ & $12 \pm 4$ & {$[5,22]$} & $9 \pm 6$ \\
\hline $\mathbf{6}$ & $9 \pm 3$ & {$[3,16]$} & $10 \pm 5$ \\
\hline $\mathbf{7}$ & $10 \pm 4$ & {$[3,19]$} & $9 \pm 6$ \\
\hline $\mathbf{8}$ & $6 \pm 2$ & {$[4,17]$} & $12 \pm 6$ \\
\hline Average & 9 & {$[4,19]$} & 10 \\
\hline
\end{tabular}

Figure 7.8 shows the average number of sensors with breathing information per $30 \mathrm{~s}$ epoch for one participant before movement removal. Intermittent high values in the number of sensors reporting breathing information, occurring at epochs 50 and 100 for example, correspond to movements. Some changes in mean loading level correspond to a position change in the participant. The vertical spikes correspond to rapid changes in loading values from the sensors, whereas the flat areas show where the sensor was loaded at a constant value, as in still breathing.

Figure 7.9 shows the raw pressure signal data, where the frequent movements are visible as pronounced 'spikes'. 


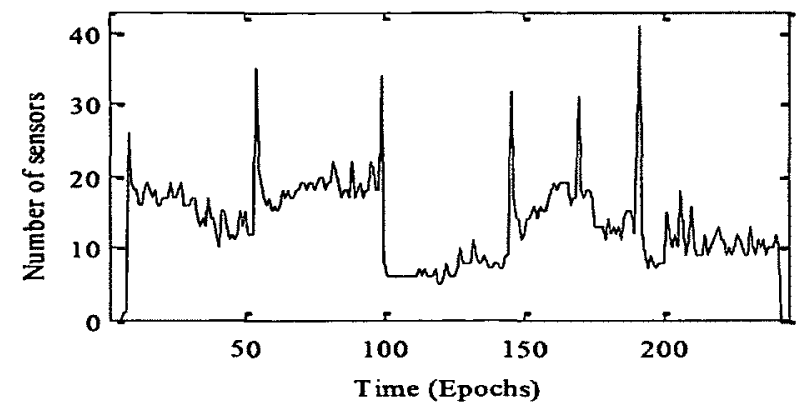

Figure 7.8 Number of sensors with breathing information

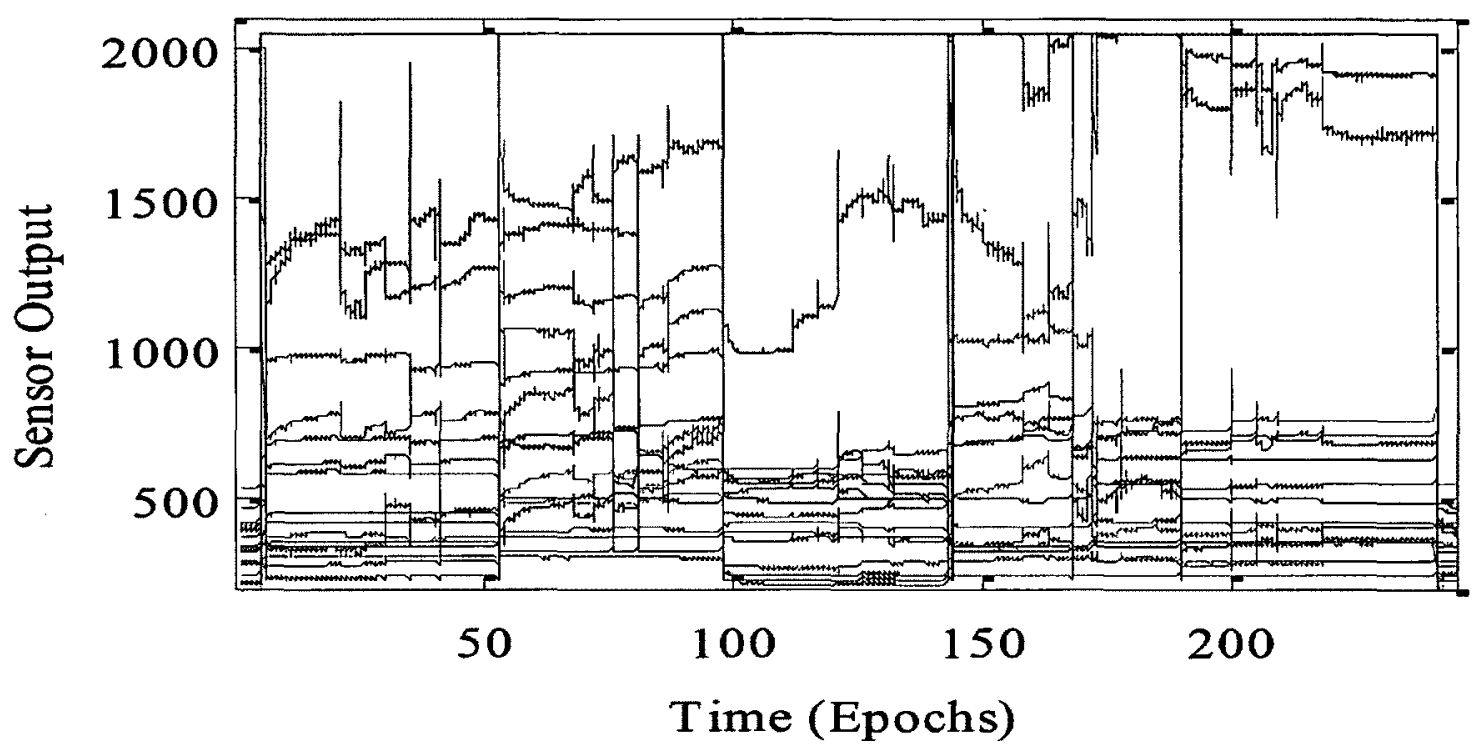

The vertical spikes correspond to rapid changes in loading values from the sensors, whereas the

flat areas show where the sensor was loaded at a constant value, as in still breathing.

Figure 7.9 Raw sensor array data

\subsubsection{Breathing Signal Quality}

Amplitude and SNR values were used to describe the signals during the alignment process and to evaluate the breathing signal produced by the algorithm. A correlation analysis was also used to evaluate the algorithm.

The SNR was calculated based on the signal's maximum spectral peak in the respiratory frequency band. This was proposed by Watanabe et al. although some adjustments have been made [47]. The SNR methodology is the contribution of Megan Holtzman, the 
second author in reference [9], which is a journal article publishing most of this chapter's content. The SNR was calculated as described by eq. (7.2)

$\mathrm{SNR}_{\mathrm{dB}}=10 \log \left(\frac{P_{\text {signal }}}{P_{\text {noise }}}\right)=10 \log \left(\frac{S_{\text {peak }}-N_{\text {peak }}}{N_{\text {bomd }}}\right)$

where $S_{\text {peak }}$ is the power at the maximum spectral peak, summed over frequency bins within the $3 \mathrm{~dB}$ lobe width of the Hanning window and $\mathrm{N}_{\text {peak }}$ is the estimated noise power summed over the same frequency bins.

This differs from Watanabe et al. 's calculation by subtracting the noise power from the peak value in the numerator to obtain only the signal power component. The denominator noise power also differs. Rather than the average noise power, noise power is represented by the total power in the respiratory frequency band $\left(\mathrm{N}_{\text {band }}\right)$. Table 7.3 presents an analysis of the array data using the strongest amplitude sensor as the reference sensor and the combined signal's properties during the alignment process.

Table 7.3 Epoch-based results for signal amplitude and SNR during signal combining steps

\begin{tabular}{|c|c|c|c|c|c|c|}
\hline \multirow[t]{2}{*}{ Participant\# } & \multicolumn{2}{|c|}{ Strongest Sensor } & \multicolumn{2}{|c|}{$\begin{array}{l}\text { With Polarity } \\
\text { Adjustment }\end{array}$} & \multicolumn{2}{|c|}{$\begin{array}{l}\text { With Delay } \\
\text { Adjustment }\end{array}$} \\
\hline & Amplitude & $\begin{array}{l}\text { SNR } \\
{[\mathrm{dB}]}\end{array}$ & Amplitude & $\begin{array}{l}\text { SNR } \\
{[\mathrm{dB}]}\end{array}$ & Amplitude & $\begin{array}{l}\text { SNR } \\
{[\mathrm{dB}]}\end{array}$ \\
\hline 1 & 10.1 & 10.8 & 37.4 & 11.0 & 42.0 & 11.1 \\
\hline 2 & 5.2 & 9.8 & 16.6 & 10.3 & 17.3 & 10.3 \\
\hline 3 & 11.1 & 9.9 & 41 & 10.0 & 43.9 & 10.1 \\
\hline 4 & 4.9 & 9.7 & 15.6 & 9.9 & 15.9 & 10.1 \\
\hline 5 & 8.4 & 9.1 & 36.6 & 9.5 & 38.4 & 9.5 \\
\hline 6 & 6.8 & 9.2 & 24.3 & 9.3 & 25.3 & 9.3 \\
\hline 7 & 6.6 & 8.7 & 25.8 & 8.7 & 26.8 & 8.8 \\
\hline 8 & 7.3 & 7.8 & 24.4 & 8.6 & 24.7 & 8.6 \\
\hline Average & 7.6 & 9.4 & 27.7 & 9.7 & 29.3 & 9.7 \\
\hline
\end{tabular}




\subsubsection{Signal Refinement}

The first plot of Figure 7.10 shows the signal waveform of the summation of all signals, without polarity adjustment. Such noisy signals often result from this combining method. The signal from the strongest amplitude sensor is shown in the second plot and the next plots show the successive signal refinement by addition of polarity adjusted signals and then delay adjustment. Clear breathing movement is seen, with successively stronger amplitudes as the signal is refined.
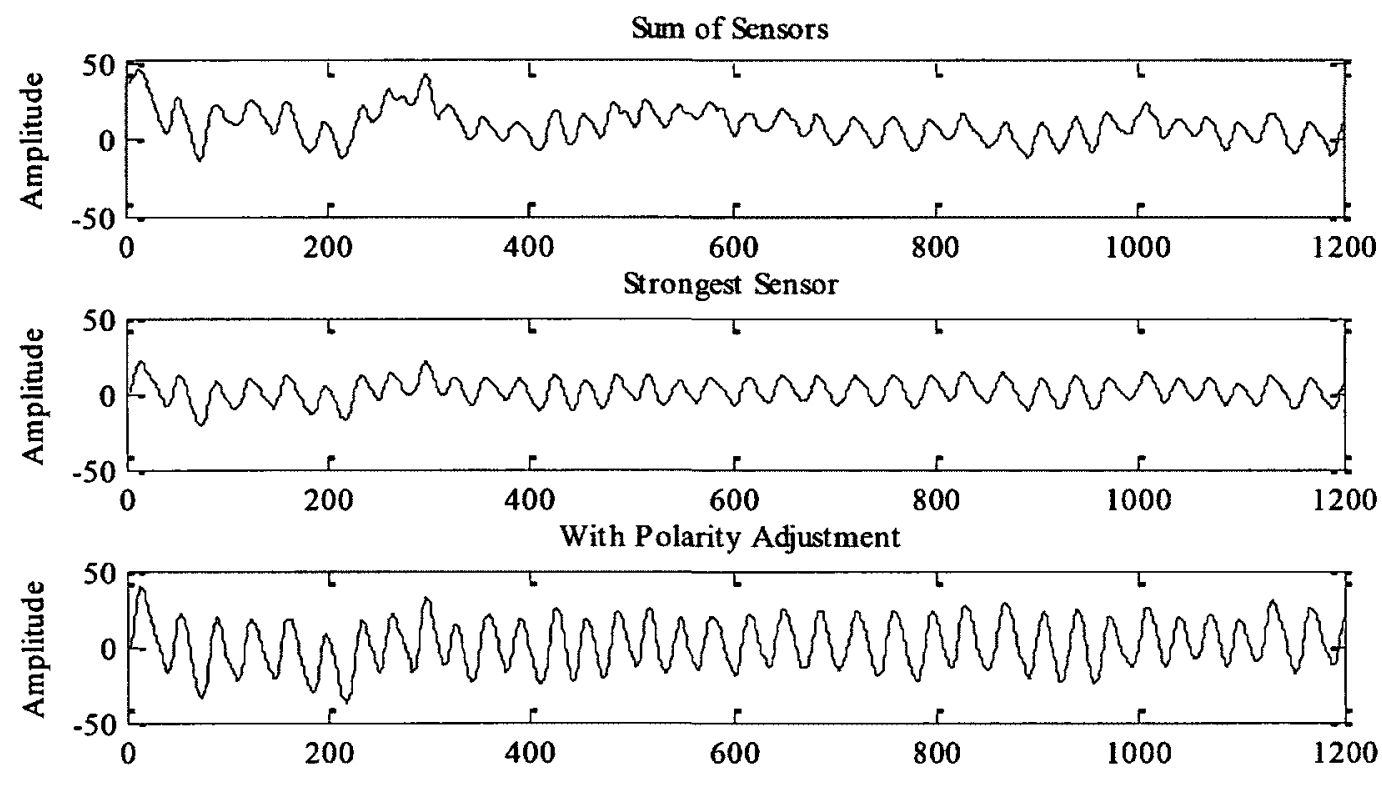

With Delay Adjustment

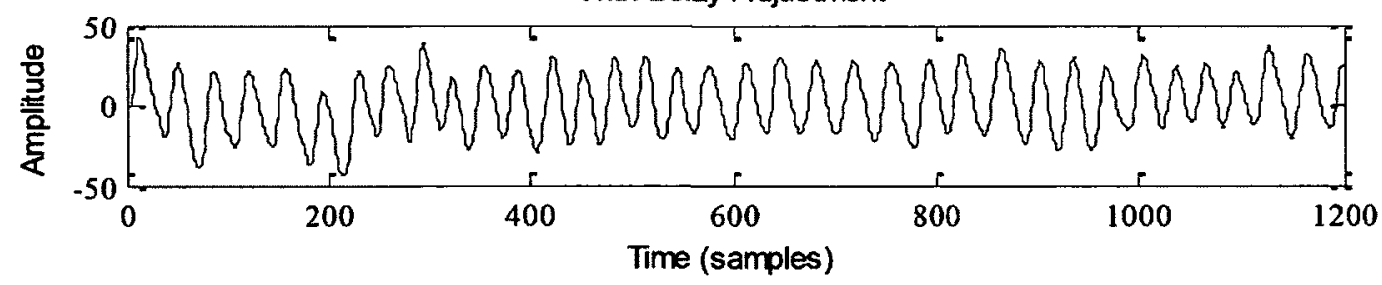

Figure 7.10 Evolution of signal strength during alignment algorithm

The histogram in Figure 7.11 shows the distribution of the number of signals which were adjusted for polarity to match that of the reference aligned signal. Given that Table 7.2 reports an average of nine sensors with breathing signals, Figure 7.11 shows that the 
mean number of inverted signals per epoch is 4.3 , which equates to approximately half of all signals. The number of polarity adjusted sensors per epoch changed with the participant's posture and position relative to the sensor array.

Figure 7.12 shows the advantage resulting from using the combining algorithm presented herein in terms of increase in breathing signal amplitude, while Figure 7.13 shows the increase in SNR for the three analyzed signals in the form of a MatLab box plot where the line in the middle of the box is the median and the box itself is defined by the $25^{\text {th }}$ and $75^{\text {th }}$ percentiles. The error-type bars indicate the most extreme data points not considered outliers. For all participants, aligning the signals increased the average epoch amplitude, while the SNR values were higher or equal in the aligned signal. The SNR increase is less noticeable than the amplitude increase, in part because in eq. 7.2 , which measures the SNR, the spectral properties of the signal are less affected by the alignment than the time domain properties.

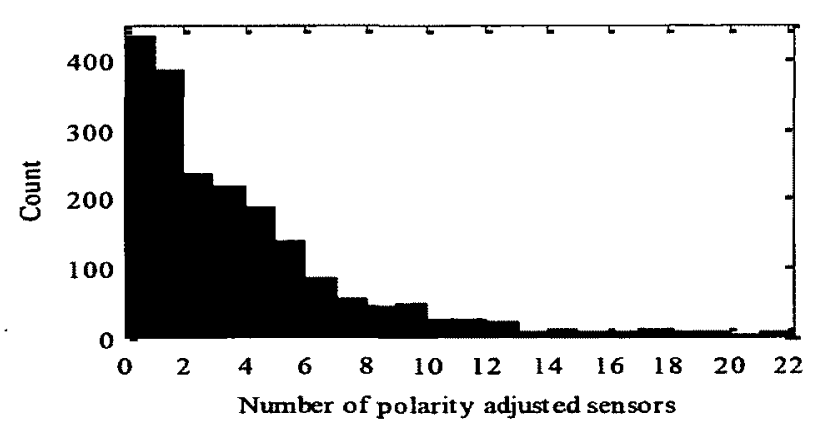

Figure 7.11 Number of polarity adjusted sensors per epoch 


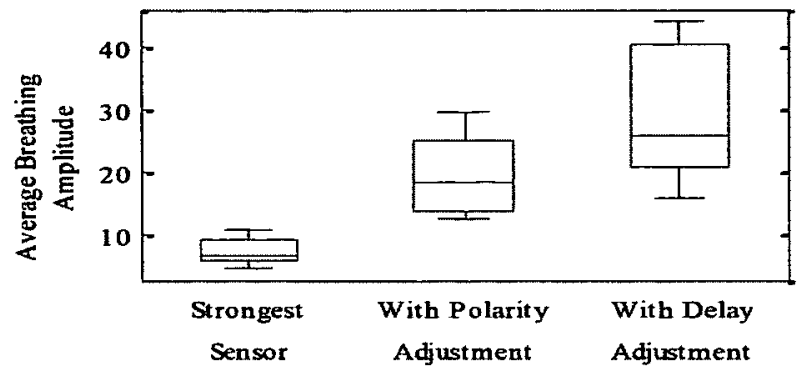

Figure 7.12 Epoch-based average breathing amplitude during signal alignment steps

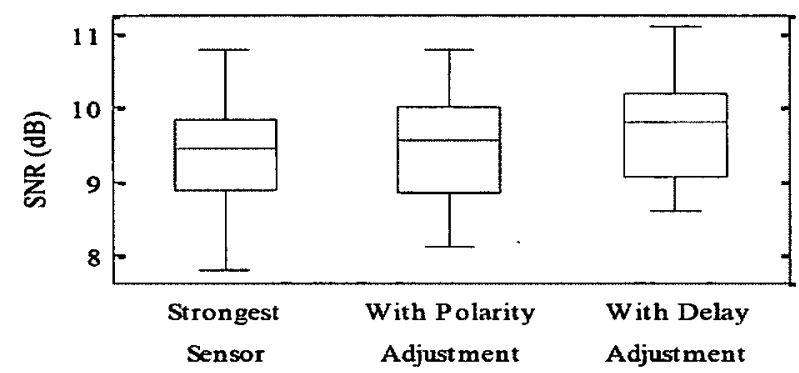

Figure 7.13 Epoch-based SNR value during signal alignment steps

\subsubsection{Comparison to Respibands}

Correlation coefficients were used to evaluate the quality of the breathing signal produced by the algorithm. Each epoch, the final pressure signal was aligned with the two respiband signals using the cross-correlation (as previously used in the algorithm). The epoch-based correlation coefficients were then calculated between the respibands themselves, and between each respiband and the final pressure signal. CEs were excluded from the calculations of Table 7.4. The measurements show average correlation coefficients between the pressure signal and the respibands to be $0.72 \pm 0.03$ and $0.68 \pm$ 0.04 , whereas the average correlation coefficient between respibands was $0.81 \pm 0.05$. 
Table 7.4 Correlation coefficients for respibands and pressure sensor

\begin{tabular}{|cccc|} 
Participant\# & $\begin{array}{l}\text { Abdominal } \\
\text { Respiband } \\
\text { and Pressure } \\
\text { Sensor }\end{array}$ & $\begin{array}{l}\text { Chest } \\
\text { Respiband } \\
\text { and Pressure } \\
\text { Sensor }\end{array}$ & $\begin{array}{l}\text { Abdominal and } \\
\text { Chest } \\
\text { Respiband }\end{array}$ \\
\hline $\mathbf{1}$ & 0.67 & 0.69 & 0.80 \\
\hline $\mathbf{2}$ & 0.76 & 0.74 & 0.82 \\
\hline $\mathbf{3}$ & 0.72 & 0.71 & 0.87 \\
\hline $\mathbf{5}$ & 0.72 & 0.69 & 0.85 \\
\hline $\mathbf{6}$ & 0.72 & 0.60 & 0.73 \\
\hline $\mathbf{7}$ & 0.73 & 0.67 & 0.78 \\
\hline $\mathbf{8}$ & 0.71 & 0.67 & 0.77 \\
\hline Average & 0.71 & 0.70 & 0.87 \\
\hline & $0.72 \pm 0.03$ & $0.68 \pm 0.04$ & $0.81 \pm 0.05$ \\
\hline
\end{tabular}

\subsubsection{Delay Analysis}

The histogram of Figure 7.14 shows the non-zero delay values used to correct the alignment of the sensor signals with breathing information for a 2 hour data sample. Delay values of 0 are most common but are not shown in order to highlight the cases where delay is indicated. Delays were markedly more positive than negative for this data, meaning that the first sensor to reach its peak reported pressure may be an indicator of a strong signal. After the signals from the array were inverted, aligned and summed, the amplitude of the combined signal was greater than that of individual sensors, which created a signal that was less complex to analyze for chest-wall movement timing. Figure 7.15 shows the combined pressure signal along with both respiband signals for three epochs. All three signals have a very similar shape. The average correlation coefficient between the delay-adjusted pressure signal and the chest respiband for the three $30 \mathrm{~s}$ epochs is 0.87 with the chest respiband and 0.88 with the abdominal respiband. 


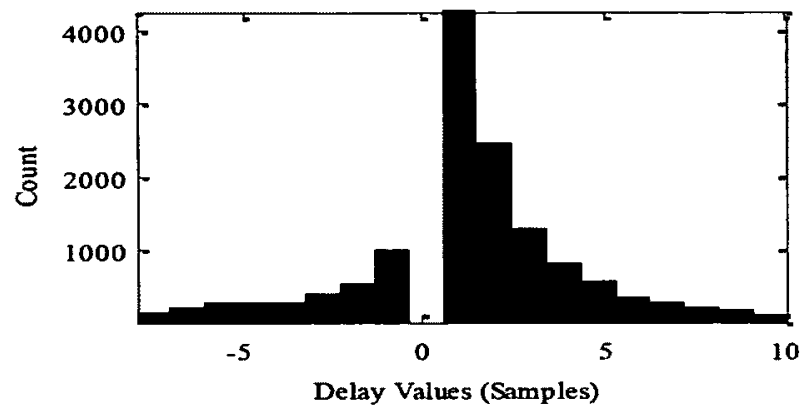

Figure 7.14 Delay values for participant data
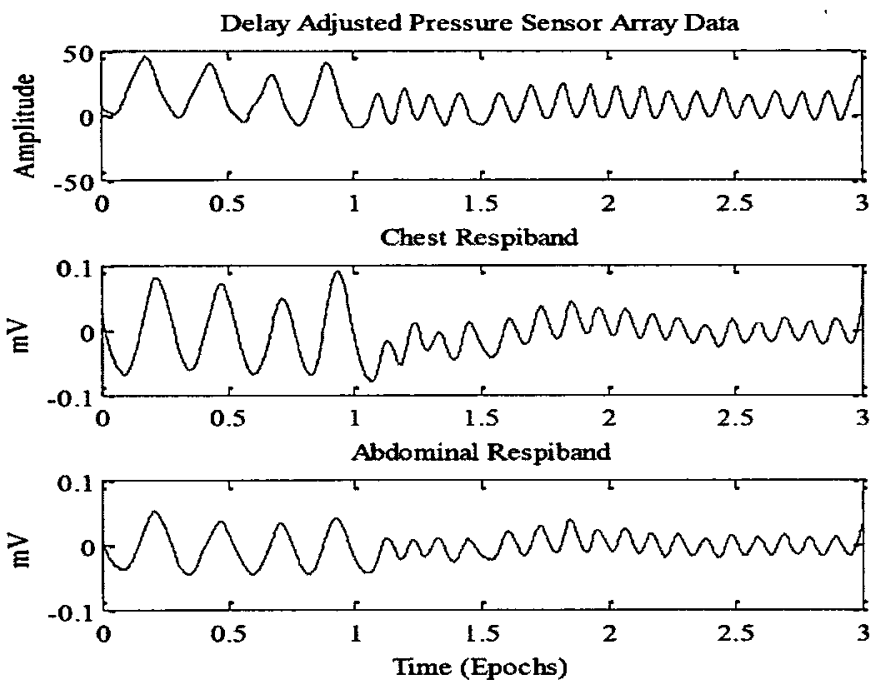

Figure 7.15 Aligned array data and respiband signals showing deep and regular breathing

\subsubsection{Pressure Image}

Figure 7.16 shows a map of the $8 \times 6$ pressure sensor grid with breathing information using color intensity to denote signal amplitude. The amplitude values, ranging from 1 to 18, were normalized. Figure 7.16 shows the strongest signal originating from the centerlower part of the right side array. A second region with strong breathing signals can be seen just above the first; these areas coincide with the ribcage and abdomen of the participant. Loaded sensors which contained no breathing information, often because the 
sensors were saturated are shown in black, as are unloaded sensors. Figure 7.17 demonstrates how the sensor with the highest amplitude, as well as the number and
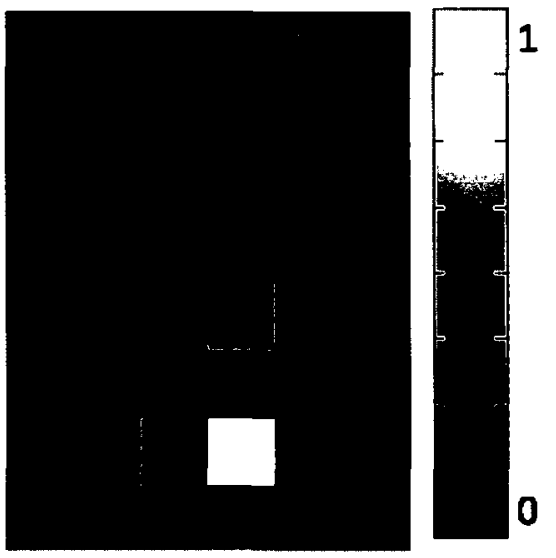

Figure 7.16 Pressure map of breathing signal amplitude with lighter colour representing higher pressure
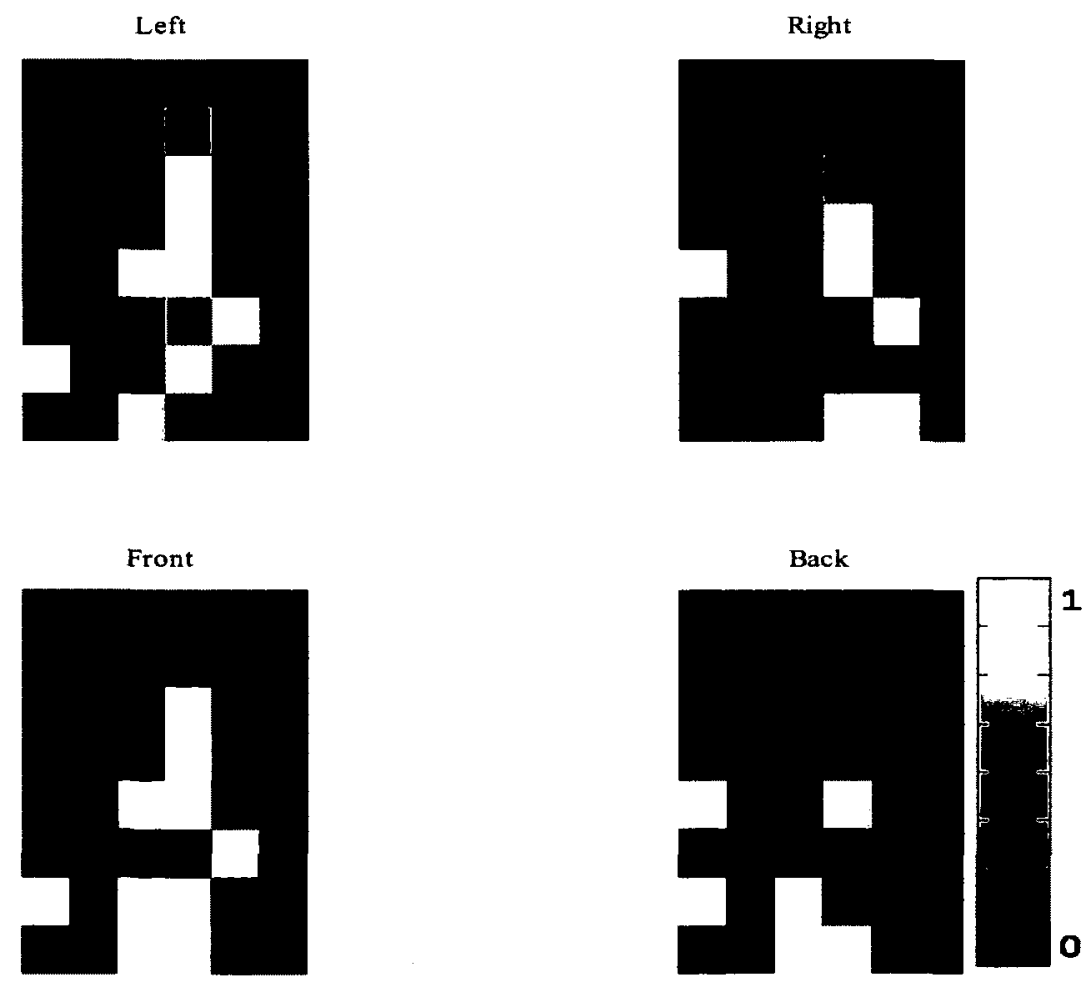

Figure 7.17 Pressure map for normalized breathing signal availability with lighter colour representing larger breathing amplitude 
distribution of the sensors containing breathing information changes with posture. The number of samples identified as containing breathing information during a 9 minute data sample for each position were summed then normalized. The colour bar next to Figure 7.17 indicates sensor locations; higher intensity (close to 1) are the sensors where breathing was detected more frequently. The four intensity images show the sensors with breathing information changing with body positions, underlining the importance of analyzing each sensor for breathing signal availability.

\subsubsection{Delay Image}

During deep breathing, some sensors which were not delayed during regular breathing incur a delay. This is best seen by mapping the delay values into the sensor grid to form a delay image. Figure 7.18 shows the filtered sensor array data for both deep and regular breathing. Figure 7.19 shows the delay profile of the array during the regular breathing (left) and during the deep breathing (right). The location of the reference sensor is shown in crosshatch. There is an area in the lower portion of the image (where more sensors are delayed in the right image than in the left image). When participants were in the supine position, this change in the delay map was common in deep breathing and less frequent in regular breathing. In Figure 7.19, during regular breathing (left), the sensors report a common delay value throughout the area identified by the box; however during deep breathing this area becomes delayed compared to the upper section of the pressure sensor array. 


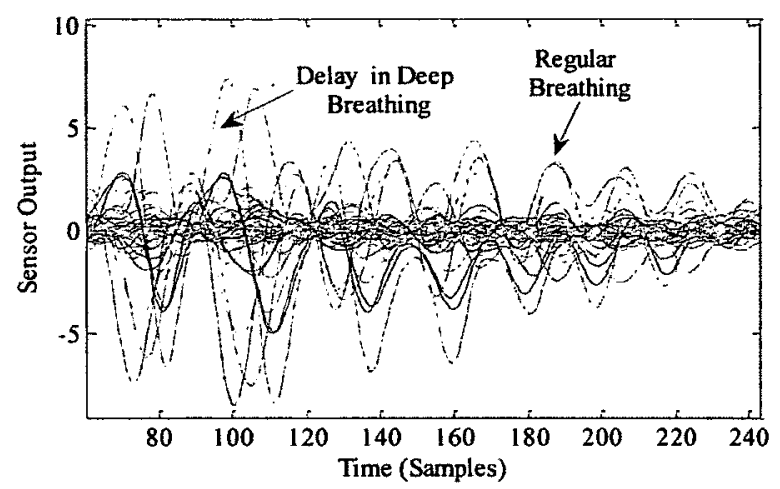

Figure 7.18 Delays between sensor outputs seen with changes in breathing depth in array data
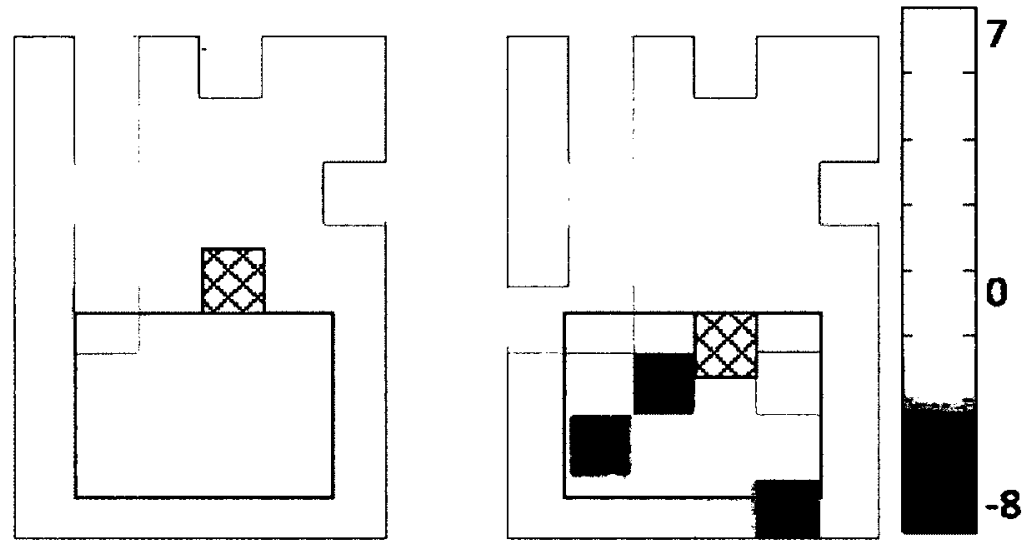

Figure 7.19 Regular breathing (left) and breathing with delay (right) with lighter colour representing positive delay and darker colour representing negative delay with respect to reference sensor (shown in crosshatch)

\subsection{Discussion}

The array data is not immediately intuitive to interpret when plotted because of the numerous signals, many of which are low in informational content (saturated, noisy, and inverted). However, it can be combined to form a breathing signal shape that is correlated strongly to both respiband signals. This chapter presented an algorithm for the combining and alignment of array data from pressure sensors placed beneath a mattress and 
capturing torso movement information. In comparison to taking the sum of all signals, selecting a single sensor provides an increase in SNR which depends on its own amplitude. Selecting a random sensor from which to extract chest motion information is problematic as a random sensor may not have breathing information or it may lose the signal after a movement. Using the listed criterion to identify breathing signals, on average, 4 to 19 of 48 sensors $(8-40 \%)$ have a useful contribution to the combined signal. Selecting the sensor with the strongest amplitude requires some processing because all sensors need to be analyzed and must be periodically reanalyzed since the strongest sensor may change after a movement or with a change in breathing depth. The additional processing required to align the signals can provide improvements in terms of signal amplitude and SNR. The choice of method employed to select the reference sensor may affect the combined signal and was investigated by Holtzman et al. in [11], a publication in which the author is also a co-author. Additionally, the criteria used to determine the presence of breathing in the signal may have led to an underestimation of the number of breathing signals per epoch.

A minimum of one sensor with breathing information was found in every epoch that was not corrupted by movement in this controlled experiment. This result is supported by a previous finding by Holtzman et al. [1] that breathing information is present in at least one sensor for every participant in that controlled experiment data. The effect of interparticipant variability can be seen in Table 7.2 where participant $\# 8$ has an average of 6 sensors available for alignment versus participant \#5 with 12 such sensors.

During regular breathing, respiband signals are phase locked, meaning the chest and abdominal curves peak at the same time, although some phase angle is expected and may 
change with aging [83] and in the presence of breathing disorders [84]. Delay mapping allows for the creation of a delay profile that presents overall torso motion. There are many applications in which the delay profile is required, for example in the case of obstructive sleep apnea where the diagnosis requires the identification of breathing motion on two areas of the torso.

Incorporating delay during sensor fusion may improve the SNR. Results from Table 7.3 show that the delay adjusted signals have the largest amplitude as measured by the peakvalley method, and have equal or higher SNR. When signals are coherent and noise is uncorrelated, alignment increases the SNR. In the case of data from the pressure sensors, the signals are not necessarily coherent: some resemble a saw tooth signal while others have a slight to moderate notch in the middle of the peak.

Additionally, the delay values may also provide information pertaining to changes in breathing depth as showed by Figure 7.18, where deep breathing caused a misalignment of the signals not seen in regular breathing. The origin of the delay between pressure sensors and respibands likely occurs because of their position relative to the participant; for example, the strongest signal may have been from the shoulders or from a local area not directly under an respiband and the physiology of breathing itself may introduce delays as normally occurs between respibands. Regions of the array under the chest and abdomen are expected to be aligned with the respective respibands. Even though the increase in the pressure array data signal SNR after aligning the delays can be small, the information is in the delay values themselves. This demonstrates the usefulness of calculating the full cross-correlation, rather than computing solely the correlation coefficient for polarity adjustment, as has been previously proposed [1]. 
To gather data respibands must be worn appropriately and connect them to the acquisition device with wires. Even wireless devices would still have to be worn properly to ensure correct data collection. The pressure array does not measure breathing only at specific points of interest, nor does it directly measure the change in ribcage circumference. However, the pressure sensor array does measure the movement of the torso, from which a respiration signal may be derived. It measures breathing without requiring special action from the participant, and when installed under the mattress as intended, it is virtually undetectable.

\subsection{Conclusions}

This chapter made use of the information captured by measuring the respiratory signal delay between sensors in the array. Cross-correlation was used to realign polarity adjusted signals from a pressure sensor array and to create a map of delays across the torso. The delay profile captured along the length and width of the sensor array reveals important information about movement timing in the torso while breathing.

Long-term sleep monitoring in the smart home context is one application for unobtrusive pressure sensor arrays. The value of unobtrusive monitoring for physiological parameters is in assessments and long-term monitoring of population groups who are at risk for respiratory events of concern without the need to actively use a biomedical monitoring instrument, a task which may exceed some patient's physical or cognitive abilities. 


\section{Chapter 8: \\ Sensor Acceptance}

\subsection{Introduction}

To increase the acceptability of long-term monitoring technologies, the perceptions of older adults towards sensor technology is investigated using a literature review to help design and implementation guidelines. Understanding behaviors that predict intentions to use and attitudes contributing to the perceived obtrusiveness of technology can help "predict acceptance of technical devices and their successful utilization [85]." This chapter validates a hypothesis regarding older adults' adoption of home monitoring technologies by conducting a literature review of articles studying older adults' attitudes and perceptions of sensor technologies ${ }^{10}$. The hypothesis states that sensor acceptance relies on a balance of privacy versus autonomy. In other words, the gain in autonomy from accepting the sensor must be equal to or outweigh the loss in privacy incurred by the sensor itself. Older adults' perceived advantages and concerns are presented in Section 8.2. The results of the literature review are presented according to study type, and sensor type in Section 8.3. The tradeoff model was applied to the results to explain the acceptance of intrusive monitoring technologies. The model created a harmonized ranking of sensors which is proposed in Section 8.4 and the chapter concludes in Section 8.5 .

\footnotetext{
${ }^{10}$ A significant portion of this chapter is published in $[13,14]$.
} 


\subsection{Literature Review}

This literature review is limited to work published in English between 2004 and 2010, including journal articles, reviews and conferences articles. IEEE Xplore and PubMed were searched with the string: (acceptance or perception* or perspective* or focus group) and (smart home or ((home monitoring or sensor network or sensor technology) and (elderly or older adult))).

The database search returned 167 articles after elimination of duplicates. Reading titles and abstracts eliminated articles not meeting the requirement for: study type (technical analysis, discussions, drug trial, and retrospective analysis), topic (single diseases, patient/doctor interaction, telecare, food safety, medication adherence, pain management and sit-to-stand approaches) or participants (doctors and caregivers, infants, adolescents, students, women $<65$, general population). Reading full papers further eliminated articles not meeting the criteria for study type, topic or participant. The exclusion criteria were chosen to focus the study on the target population of older adults who experience a broad range of biomedical monitors. As shown in Figure 8.1, 18 articles were included in the analysis.

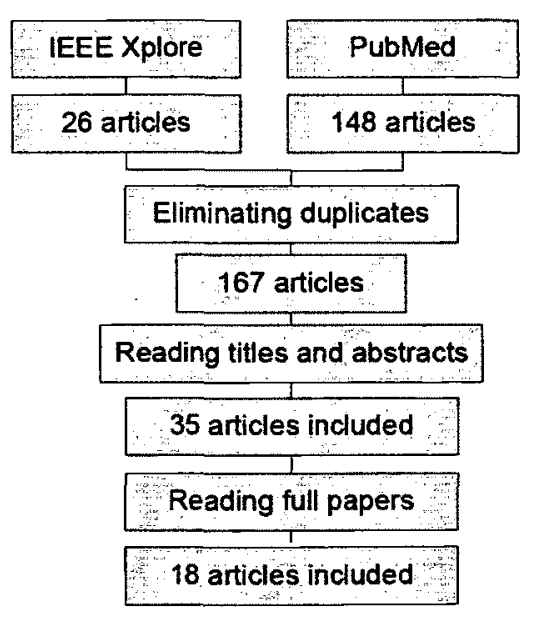

Figure 8.1 Method used in selecting articles for literature review and synthesis 
Table 8.1 displays the number of participants in each focus group and pilot study. In total, 14 focus group studies and 5 pilot studies were included. One article included both study types [86]. On average, the number of participants in the focus group was larger than in the pilot studies (average of 19 vs. 12 participants). Few studies explicitly referenced cost or time as limiting the study size, though the cost for equipment and time investments are significantly larger for pilot studies.

Table 8.2 indicates which types of sensors were included in focus groups and pilot studies when stated explicitly. Wearable sensors were presented for location and physiological monitoring. One focus group presented implanted physiological and location monitoring chips to participants. Environmental sensors included switches, stove temperature sensors, video and infrared cameras, bed occupancy and bed-based heart rate along with respiration monitoring type sensors. Few focus groups presented implanted physiological and location monitoring chips to participants.

Table 8.1 Type of study and number of participants for articles included in literature review

\begin{tabular}{|l|l|l|}
\multicolumn{2}{c|}{$\begin{array}{l}\text { Number of } \\
\text { participants }\end{array}$} \\
\hline Focus Group & $21-30$ & {$[87],[88],[89],[90]$} \\
& $10-20$ & {$[19],[91],[92],[93],[94],[95],[96],[97]$} \\
& $1-10$ & {$[86],[98]$} \\
\hline \hline Pilot & $21-30$ & {$[99]$} \\
& $11-20$ & {$[20]$} \\
& $1-10$ & {$[86],[100],[101]$} \\
\hline
\end{tabular}

Table 8.2 Type of sensor presented in focus group or used in pilot

\begin{tabular}{|l|l|ll|}
\hline Focus Group & {$[19],[87],[88],[89]$,} & {$[92],[96]$} & {$[19],[86],[87],[89],[90],[91]$,} \\
& {$[90],[92],[93],[97]$} & {$[92],[93],[94],[95],[98],[96]$,} \\
& & {$[97]$} \\
& & \\
\hline \hline Pilot & {$[20],[99],[100],[101]$} & {$[20],[86]$} \\
\hline
\end{tabular}


The potential advantages to smart home monitoring foreseen by potential users of such systems are listed in Table 8.3. The concerns elucidated by participants about the monitoring technology were extracted from all 18 articles and classified into themes which were found to be popular in the literature. They are presented in

Table 8.4. This review was limited by keywords, study topic and participant age in order to best investigate support for the tradeoff hypothesis.

Table 8.3 Potential advantages to home monitoring sensors technologies as reported by participants

\begin{tabular}{|lll|}
\hline & Focus Group & Pilot \\
\hline Autonomy & {$[20],[88],[92]$} & \\
\hline Cognitive Health & {$[20],[88],[89]$} & {$[99]$} \\
\hline Social Contact & {$[89],[90]$} & {$[101]$} \\
\hline Socially Desirable & {$[89]$} & {$[86]$} \\
\hline Information Sharing & {$[88],[89],[92],[95]$} & {$[20],[86],[99]$} \\
\hline $\begin{array}{l}\text { Usefulness Safety, } \\
\text { Emergency, Falls }\end{array}$ & {$[88],[90],[92],[93],[94],[95],[97]$} & {$[20],[86],[101]$} \\
\hline
\end{tabular}

Table 8.4 Participant concerns classified in themes

\begin{tabular}{|lll|}
\hline \multicolumn{1}{l}{ Focus Group } & Pilot \\
\hline Physical & {$[19],[87],[90],[92],[95]$} & {$[86],[99],[100],[101]$} \\
\hline $\begin{array}{l}\text { Human } \\
\text { Interaction }\end{array}$ & {$[87],[88],[90],[92],[93],[94]$} & {$[20]$} \\
\hline Usability & {$[87],[90],[92],[93],[94]$} & {$[99],[100],[101]$} \\
\hline Stigma & {$[19],[87],[92],[93],[95],[98]$} & \\
\hline Privacy & {$[19],[87],[88],[89],[90],[91],[92],[93],[95]$,} & {$[101]$} \\
& {$[98]$} & \\
\hline Routine & {$[89],[92]$} & {$[20],[99],[101]$} \\
\hline Function & {$[87],[90],[92],[95]$} & {$[20],[86],[100]$} \\
\hline Cost & {$[87],[90],[92],[93]$} & \\
\hline
\end{tabular}

\subsection{Results}

Comparisons made between at least two types of sensors were collected from the papers and are presented in Table 8.5, a ranking of sensors according to the information they capture. A number indicates the sensor preference reported by participants, with the 
number 1 indicating the most preferred. Explicit and implicit rankings were available in half of the retained papers. Comparison presented between at least two types of sensors are presented in the table below with sensors divided into four categories according the amount of private information they collect to represent the level of privacy traded: On/off (e.g. user activated health line alarms), Intermittent physiological information (e.g. blood pressure monitoring cuff), Continuous Physiological (CP) information (e.g. wearable heart rate monitor), Activities of daily living (ADL) (e.g. sensors in bathroom and kitchen), Location and position (L\&P) (e.g. computer vision for fall detection), and Complete Visual information (VC) (e.g. video camera).

Table 8.5 shows the ranking of levels of information which can be captured by smart home monitoring technologies along with numbers indicating how they were ranked by focus group or pilot participants.

Table 8.5 Sensor preferences as reported by participants and harmonized ranking

\begin{tabular}{|llllll|}
\hline$[20]$ & On/Off & CP & ADL & L\&P & VC \\
\hline$[90](40-59)$ & 1 & 2 & & & \\
\hline$[90](65+)$ & 1 & 1 & 4 & 3 & 5 \\
\hline$[91]$ & & 2 & 4 & 3 & 5 \\
\hline$[92]$ & 1 & 1 & 1 & 1 & 2 \\
\hline$[93]$ & 1 & 2 & 4 & 3 & 5 \\
\hline$[95]$ & & 1 & & 2 & 3 \\
\hline$[98]$ & & & & 1 & 2 \\
\hline$[96]$ & & 1 & & 1 & 2 \\
\hline$[97]$ & & 1 & & 2 & 2 \\
\hline$[101]$ & 1 & 2 & & & \\
\hline $\begin{array}{l}\text { Harmonized } \\
\text { ranking }\end{array}$ & 1 & 2 & 4 & 3 & 5 \\
\hline
\end{tabular}




\subsection{Discussion}

\subsubsection{Tradeoff Model: Privacy versus Autonomy}

Trends in Table 8.5 allow the creation of a harmonized ranking which was created by determining the global trends in sensor preference. The ranking is as follows from lowest to highest level of information captured: On/off, CP, L\&P, ADL, VC, which is congruent with $9 / 10$ of the papers containing sensor rankings. As the acceptability of sensors decreases, the information captured by the sensors becomes increasingly revealing. It is hypothesized that the gain from accepting the sensor technology must be bigger to be traded against the loss of privacy and intrusion of the sensor. The tradeoff for the helpline (on/off type sensor) is getting to the emergency room more quickly. There is little loss of privacy as the user has full control of the sensor and a little gain in autonomy from being able to activate it. The $\mathrm{CP}$ and intermittent sensors prevent wearers from having to go to the clinic as often for physiological monitoring and thus a slight loss of privacy is traded for a slight gain in autonomy. In the case of L\&P monitoring, there is a moderate loss of privacy and a moderate gain in autonomy. In the case of the ADL monitor, the older adult is able to avoid home visits or going to a retirement home. This monitor confers a moderate to high loss of privacy by way of continuous monitoring however there is a moderate gain in autonomy. VC type sensors have a high loss of privacy and a moderate gain in autonomy hence ranked last. Participants in many studies stated they would accept the use of video monitoring if it allowed them to stay in their homes longer [19], [20]. Participants felt the privacy intrusion was tolerable in exchange for feeling safe in their own homes [92]. 
The privacy versus autonomy tradeoff model can explain sensor acceptance in older adults. For example at the less intrusive end of the spectrum, an older adult at risk of stroke would likely accept intermittent use of a wearable blood pressure device, but not video cameras throughout the home. An older adult at risk of falls may be willing to accept the more intrusive monitoring. In this example, a bigger loss in privacy is traded to avoid not being found quickly after a fall. Older adults in this case would not likely accept video monitoring if a less obtrusive sensor is adequate. At the most obtrusive level, the privacy lost from accepting video cameras would only be acceptable if it could prevent transfer to a long-term care facility which represents the greatest loss in autonomy. The literature contains numerous examples of the extreme case of trading all privacy (allowing video cameras in the home) to prevent a long-term care placement [87], [88], [90].

The autonomy associated with aging-in-place is valued higher than privacy. The loss of autonomy associated with placement in a nursing home was felt to be worse than the loss of privacy associated with all types of sensors.

Coughlin et al.'s focus group [87] was in agreement that loss of privacy $24 / 7$ "cost the individual too much unless they are extremely frail or the only other alternative may be nursing care or living with an adult child." This was supported by Mihailidis et al. who identified the tradeoff of "the perceived invasion of privacy versus perceived risk of injury" $[90]$.

Across both study types, 56 of 88 (64\%) participants opposed the use of video cameras for monitoring because it was a violation of their privacy [20],[90],[91],[92],[93],[95]. Additionally 'some' or 'up to half' expressed privacy concerns [19],[87],[89], [98]. The 
infrared cameras used as proximity sensors in Demiris et al. were seen as less intrusive and more acceptable [86]. Adults 70-74 yrs old reported feeling more comfortable with a modification made to video capture, that rendered it unidentifiable (creating a silhouette image), than with regular video [90], as did older adults in Demiris et al. [93].

\subsubsection{Validation of the Tradeoff Model}

There are many factors which may lead an older adult to accept home monitoring technologies (aging-in-place, increased cognitive and physical safety, information transfer to circle of care), and factors which direct them against acceptance (cost, privacy and technological burden).

The tradeoff model of privacy versus autonomy for sensor acceptance simplifies the decision to one dimension. A decision making model can be validated by recruiting older adults and presenting them with a questionnaire. To validate the harmonized sensor ranking, the questionnaire would present different disease levels and monitoring technologies and ask the participant which type of information they would provide (reduction in privacy by way of sensor) in exchange for maintaining the current level of autonomy.

Applied to the pressure sensor array used in this thesis, the privacy vs. autonomy tradeoff model suggests that the array, as a CP sensor, would be accepted by older adults. The tradeoff model can be applied to the specific case of video cameras. The value of the video monitoring service was thought to be more important if the use of such devices allowed them to remain in the home. Applying the trade-off model, the literature synthesis suggest that older healthy adults would likely not accept video surveillance in 
any situation, however frail older adults might be willing to "trade in their privacy" if it meant preventing nursing home placement.

Many older adults stated that they would acquire or use a device if they thought they needed it. However, one third of focus groups reported at least one participant with the difficulty of perceiving themselves as benefiting or even requiring smart home technology in the future [87],[88],[95],[98]. Courtney et al. provides a list of nine factors affecting the perception older adults' for the need for the technology, and noted that family, caregivers and health care provider's assessment of an older adults' need was not consistent with their own [98]. These older adults associated smart home technologies with frailty and stigma. Coughlin et al. reported "few adults of any age see themselves as 'old' and even fewer as "frail"' and that technologies, especially wearable monitors, would be a stigma to frail adults rather than taking charge of their health and independence [87]. This is supported by Wild et al.'s finding that participants had an “inability to anticipate one's own cognitive decline" [88].

Participants in one focus group that reported privacy concerns when discussing the concept of video monitoring had reduced concerns when assuming that there was a proven need for monitoring with video [98]. Courtney showed that older adults' "own perceptions of their need for the technology can override their privacy concerns" [19].

\subsubsection{Challenges Comparing Focus Group and Pilot Results}

The questionnaires presented to pilot participants were not published in the five pilots retained for the review, though they may be published in a separate article. Information presented to focus groups to elicit responses was published alongside results in Wild et 
al. [88] and Beaudin et al. [89], and most focus group studies discussed in depth the methodology and topics covered.

Privacy concerns were reported in 9 of 12 focus groups compared to only 1 of 5 pilots. Two of sixty individuals participating in pilots withdrew from studies (none from focus groups): one due to obtrusiveness of video camera [20] and the other because they suffered a stroke during the pilot and experienced problems using the wearable device [100]. Perhaps the concept of monitoring conjures thoughts on the surveillance end of the spectrum rather than 'just checking in', and that the negative feelings towards surveillance are more likely to surface in focus groups where there is not actual firsthand experience to ground these perceptions. Coughlin et al. suggest that the "digital divide between adopters and non-adopters of technology," the user's mental model of how technology should work [87] and education in technology to explain barriers to use and initial suspicion. This difference in the prevalence of privacy concerns may be due to difficulties in conceiving technology without interacting with it.

\subsubsection{Challenges in Defining "Obtrusive"}

The definition of unobtrusive, invasive, passive, active and nonintrusive sensors for monitoring differs depending on the application and in comparison to the invasiveness of the traditional clinical approach. Courtney et al. [102] and Hensel et al. [103] defined obtrusiveness as having a quality that is "perceived as undesirable and physically and/or psychologically prominent" and identified eight dimensions affected from privacy, cost, fear of embarrassment, to the effect on relationships. 'Obtrusive' is used differently because it is context dependent. Obtrusive compared to not having the device is different to obtrusive when considering device options. For example a home telemedicine center 
with video camera was so obtrusive that no participant wanted to use it, but when a wooden box with a picture frame was put on it when not in use the same technology was accepted by $95.5 \%$ of participants [104]. Compared to the contact sensors used in PSG and to the proposed alternate sensor, the pressure sensor arrays used in this thesis can be considered unobtrusive because they are non-contact, dissimulated within furniture and do not require user interaction once installed. The sensor is also unobtrusive in that neither picture nor sounds are collected.

\subsection{Conclusions}

Sensors and biomedical monitoring were consistently seen as positive when supporting aging-in-place. Maintaining independence is a driving factor for sensor acceptance. Numerous examples of older adults circumventing the use of medical devices such as alert pendants, hearing aids, fall sensors and cameras abound in the literature. Understanding which factors of monitoring affect acceptance can help reduce intentional non-compliance. This chapter highlights the overall positive attitude of older adults towards unobtrusive sensor technologies in smart homes when they perceive the need and whenever they support autonomy. The tradeoff model was applied to sensor acceptance and describes how as the information captured by the sensor becomes more intrusive and the infringement on privacy increases, sensors are accepted if the loss in privacy is traded for an equal amount of autonomy. 


\section{Chapter 9: Conclusions}

\subsection{Thesis Conclusions}

This chapter concludes the thesis. Thesis conclusions are presented in section 9.1 and thesis contributions are presented in section 9.2. Finally, section 9.3 presents areas for continuing work to be completed by future students.

The value in unobtrusive sensors for sleep and apnea monitoring is that they require no user interaction and can collect long-term health information without modifying the subject's behavior. This is especially relevant for patients with cognitive difficulties or patients who do not want to wear monitoring devices. The pressure sensor array used in this thesis lies underneath the mattress to detect movement and respiration. Advantages stemming from its unobtrusive placement are increased compliance with monitoring protocol and the ability to monitor people at home. The pressure sensor array used in these experiments and the experimental setup was described in chapters 2 and 3 . The unobtrusive sensor used herein is less expensive and more widely applicable than standard full polysomnography and can detect rollovers and central apneas in a variety of home-based contexts. To understand which factors affect the acceptance of sensor technologies in smart homes, a model was proposed after synthesizing current constructs of attitudes towards monitoring technologies. 


\subsection{Thesis Contributions}

A sleep monitoring system was presented in Chapter 1 , which system integrates datadriven context and static patient-driven context. The experimental setup and verification was conducted in Chapter 3, validation result were published in [1]. For example, lying position and context-aware error correction are used in Chapter 4 to reduce false positive detections in order to adapt apnea detection algorithms. The system also makes use of static patient-driven context in Chapter 5 to screen patients who do not meet the BMI criteria for the sensor [6]. These algorithms along with two others for apnea detection and one focusing on rollover detection presented in Chapter 6 have been published in [2], [3], [4], [5], and [8]. Two sensor arrays were used for data collection and interpreted as a low resolution image in Chapter 7 . To do so the delay profile was created for the sensor during regular and deep breathing as well as for different lying positions. The delay mapping was published in [9] with preliminary results also presented in [10]. Preliminary work focusing on methods for choosing a reference sensor has been submitted [11]. The acceptability of sensor technologies for smart home monitoring was studied in Chapter 8 and ranked in terms of acceptability according to the developed Privacy versus Autonomy tradeoff model. The model was proposed and published in [13], with preliminary results presented separately [14].

\subsection{Future Work}

Sleep monitoring is a growing and very interesting area of research. There are many paths of work for future students, which lay outside the scope of this thesis. Some of these are listed below. 
1. The delay profile analysis in chapter 7 presented the initial work towards the identification of obtrusive apneas using an analysis of region of interests on the pressure view as well as the measurement of delays found between the sensor elements of the array. Many patient files collected from the West Ottawa Sleep Centre present obstructive apneas and could be analyzed for the presence of these events. This involves significant data analysis and processing work to remove movements and possibly determine a preliminary dataset of typical apneas. Controlled experiments where healthy volunteers simulate OAs may be helpful in determining possible signal morphologies.

2. Design and prepare validation of sensor acceptance model. Conducting a pilot test or focus group to validate the sensor acceptance model is outside the scope of this thesis. The research protocol could propose a pilot study at the Smart Apartment at the Elizabeth Bruyère Hospital. This work would entail the drafting of an informed consent form, letter to participants, recruitment notices and participant questionnaire to be completed after a visit in the Smart Apartment. The pilot study protocol could require participants to visit and make use of the Smart Apartment for a few hours or overnight to obtain their impressions about the various monitoring technologies available.

3. The pressure sensor arrays produce great quantities of information, as do the numerous algorithms developed to interpret its data. A major factor limiting the dissemination of this technology is the lack of a comprehensive and 'low-effort' interface. An ideal interface could make use of WebGL, which is a $3 D$ modeling technology available through a web browser such as Google Chrome. An application 
could be built to present the smart home occupant as an avatar, with the avatar mimicking breathing and movement motion as detected by the pressure sensor array and its algorithms. This type of modeling and data visualization may make the monitoring technology more accessible.

4. Validate rollover detection algorithm with PSG data. This requires a manual annotation of rollovers and of many other movements from a number of overnight recording files which are approximately 8 hours long. The pressure sensors were oriented differently for the sleep laboratory data collection than they were in the controlled experiments for rollover detection. As such, the feature extraction and center of gravity equations must be modified. Upon visual inspection, rollovers in the PSG data appear different than in the controlled experiments, and new features may be required to obtain a high correct classification rate.

5. Determine how rollovers and posture changes correlate to sleep state. Rollovers were used by Miwa et al. in [28] to infer deep vs. light sleep as determined by the algorithm of wrist worn actigraphy. This methodology could be improved by comparing rollovers to the actual sleep state determined by the polysomnographers interpreting the overnight EEG recording. Rollover proximity to REM sleep and to awakenings could be analyzed. This allows some level of correlation measurement between the presence or frequency of rollovers and the sleep stage. This analysis makes use of PSG data and calculates the correlation measurement for a group of patients or for individual patients. 


\section{References}

[1] M. Holtzman, D. Townsend, R. Goubran and F. Knoefel, "Validation of pressure sensors for physiological monitoring in home environments," in Proc. IEEE Int. Workshop Med. Meas. Appl., 2010, pp. 38-42.

[2] D. Townsend, M. Holtzman, R. Goubran, M. Frize and F. Knoefel, "Simulated central apnea detection using the pressure variance," in Proc. 31st Annu. Int. Conf. IEEE EMBS, 2009 , pp. 3917-3920.

[3] D. Townsend, M. Holtzman, R. Goubran, M. Frize and F. Knoefel, "Effect of windowing on central apnea detection," in Proc. IEEE Int. Workshop Med. Meas. Appl., 2010 , pp. 117-120.

[4] D. Townsend, M. Holtzman, R. Goubran, M. Frize and F. Knoefel, "Relative Thresholding with Under Mattress Pressure Sensors to Detect Central Apnea," IEEE Trans. Instrum. Meas., vol. 60, pp. 3281 - 3289, Oct. 2011.

[5] D. Townsend, R. Goubran and F. Knoefel, "Amplitude-based central apnea screening," in Proc. IEEE Int. Symp. Med. Meas. Appl., 2011, pp. 395-398.

[6] D. Townsend, R. Goubran, F. Knoefel and J. Leech, "Validation of Unobtrusive Pressure Sensor Array for Central Sleep Apnea Screening," IEEE Trans. Instrum. Meas., vol. 61, Jul. 2012.

[7] D. Townsend, J. Leech, F. Knoefel and R. Goubran, "Clinical Investigation into the Use of an Under Mattress Pressure Sensor in the Detection of Central Apneas," 26th Annual Meeting of the Associated Professional Sleep Societies, SLEEP, 2012.

[8] D. Townsend, R. Goubran, M. Frize and F. Knoefel, "Preliminary results on the effect of sensor position on unobtrusive rollover detection for sleep monitoring in smart homes," in Proc. 31st Annu. Int. Conf. IEEE EMBS, 2009, pp. 6135-6138.

[9] D. Townsend, M. Holtzman, R. Goubran, M. Frize and F. Knoefel, "Measurement of Torso Movement With Delay Mapping Using an Unobtrusive Pressure-Sensor Array," IEEE Trans. Instrum. Meas., vol. 60, pp. 1751-1760, May. 2011.

[10] D. Townsend, R. Goubran, M. Frize and F. Knoefel, "Measuring chest movement using an array of unobtrusive pressure sensors," in Proc. 27th Annu. Int. Conf. IEEE IMTC, 2010, pp. 1053-1056.

[11] M. Holtzman, D. Townsend, R. Goubran and F. Knoefel, "Breathing sensor selection during movement," in Proc. 33rd Annu. Int. Conf. IEEE EMBS, 2011, pp. 381-384. 
[12] D. Townsend, R. Goubran and F. Knoefel, "Time domain characterization of window length and type on moving variance signal features," in Proc. IEEE Int. Symp. Med. Meas. Appl., 2012,

[13] D. Townsend, F. Knoefel and R. Goubran, "Privacy versus autonomy: A trade off model for smart home monitoring technologies," in Proc. 33rd Annu. Int. Conf. IEEE EMBS, 2011, pp. 4749-4752.

[14] D. Townsend, F. Knoefel and R. Goubran, "Home Video Monitoring: A Tradeoff Between Privacy and Autonomy," IEEE Ist Annu. Int. Conf. WIE, 2011.

[15] National Institutes of Health, "2003 national sleep disorders research plan," U.S. Department of Health and Human Services, 2003.

[16] T. Young, et al, "Burden of sleep apnea: rationale, design, and major findings of the Wisconsin Sleep Cohort study," WMJ, vol. 108, pp. 246-249, Aug. 2009.

[17] American Association of Sleep Medicine, "Obstructive Sleep Apnea," 2008, http://www.aasmnet.org/Resources/FactSheets/SleepApnea.pdf.

[18] P. Bartolomeu, J. Fonseca and F. Vasques, "Challenges in health smart homes," in Proc. 2nd Int. Conf. Pervasive Computing Technologies for Healthcare, 2008, pp. 19-22.

[19] K. L. Courtney, "Privacy and senior willingness to adopt smart home information technology in residential care facilities," Methods Inf. Med., vol. 47, pp. 76-81, 2008.

[20] A. Essen, "The two facets of electronic care surveillance: an exploration of the views of older people who live with monitoring devices," Soc. Sci. Med., vol. 67, pp. 128-136, Jul. 2008.

[21] D. M. Mahoney, P. H. Mutschler, B. Tarlow and E. Liss, "Real world implementation lessons and outcomes from the Worker Interactive Networking (WIN) project: workplace-based online caregiver support and remote monitoring of elders at home," Telemed. J. E. Health., vol. 14, pp. 224-234, Apr. 2008.

[22] M. H. Kryger, T. Roth and W. C. Dement, Principles and Practice of Sleep Medicine. ,4th ed.Philadelphia: Elsevier/Saunders, 2005, pp. 1517.

[23] P. Johansson, U. Alehagen, E. Svanborg, U. Dahlström and A. Broström, "Sleep disordered breathing in an elderly community-living population: Relationship to cardiac function, insomnia symptoms and daytime sleepiness," Sleep Med., vol. 10, pp. 1005$1011,2009$.

[24] F. Giganti, G. Ficca, S. Gori and P. Salzarulo, "Body movements during night sleep and their relationship with sleep stages are further modified in very old subjects," Brain Res. Bull., vol. 75, pp. 66-69, Jan. 2008. 
[25] N. Wolkove, O. Elkholy, M. Baltzan and M. Palayew, "Sleep and aging: 1. Sleep disorders commonly found in older people," CMAJ, vol. 176, pp. 1299-1304, Apr. 2007.

[26] E. L. Hill, R. G. Cumming, R. Lewis, S. Carrington and D. G. L. Couteur, "Sleep Disturbances and Falls in Older People," J. Gerontol. A Biol. Sci. Med. Sci., vol. 62, pp. 62-66, 2007.

[27] I. Szollosi, T. Roebuck, B. Thompson and M. T. Naughton, "Lateral sleeping position reduces severity of central sleep apnea / Cheyne-Stokes respiration," Sleep, vol. 29, pp. 1045-1051, Aug. 2006.

[28] H. Miwa, S. I. Sasahara and T. Matsui, "Roll-over detection and sleep quality measurement using a wearable sensor," in Proc. 29th Annu. Int. Conf. IEEE EMBS, 2007, pp. 1507-1510.

[29] C. Iber, S. Ancoli-Isreal, A. L. Chesson and S. F. Quan, The AASM Manual for the Scoring of Sleep and Associated Events: Rules, Terminology and Technical Specifications. Westchester, IL: The American Academy of Sleep Medicine, 2007,

[30] A. Rechtschaffen and A. Kales, A Manual of Standardized Terminology, Techniques, and Scoring Systems for Sleep Stages of Human Subjects. Los Angeles: Brain Information/Brain Research Institute, 1968,

[31] K. Banno and M. H. Kryger, "Sleep apnea: clinical investigations in humans," Sleep Med., vol. 8, pp. 400-426, Jun. 2007.

[32] W. W. Flemons, N. J. Douglas, S. T. Kuna, D. O. Rodenstein and J. Wheatley, "Access to diagnosis and treatment of patients with suspected sleep apnea," Am. J. Respir. Crit. Care Med., vol. 169, pp. 668-672, Mar. 2004.

[33] C. F. George, "Reduction in motor vehicle collisions following treatment of sleep apnoea with nasal CPAP," Thorax, vol. 56, pp. 508-512, Jul. 2001.

[34] A. Yoshino, et al, "Risk factors for traffic accidents in patients with obstructive sleep apnea syndrome," Sleep and Biol. Rhythms, vol. 4, pp. 144-152, June. 2006.

[35] Canadian Medical Association, Determining Medical Fitness to Operate Motor Vehicles CMA Driver's Guide. ,7th ed.2006, pp. 127.

[36] Ministry of Health and Long-Term Care, "Assisted Devices Program," May 5th 2011. http://www.health.gov.on.ca/en/public/publications/pub adp.aspx.

[37] C. Jilek, et al, "Prognostic impact of sleep disordered breathing and its treatment in heart failure: an observational study," European Journal of Heart Failure, vol. 13, pp. 68-75, Jan. 2011. 
[38] J. Cohen, "A coefficient of agreement for nominal scales," Educational and Psychological Measurement, vol. 20, pp. 37-46, 1960.

[39] P. S. Jensen, H. B. Sorensen, H. L. Leonthin and P. Jennum, "Automatic sleep scoring in normals and in individuals with neurodegenerative disorders according to new international sleep scoring criteria," J. Clin. Neurophysiol., vol. 27, pp. 296-302, Aug. 2010 .

[40] C. W. Whitney, et al, "Reliability of scoring respiratory disturbance indices and sleep staging," Sleep, vol. 21, pp. 749-757, Nov. 1998.

[41] M. Suzuki, et al, "Discrepancy in polysomnography scoring for a patient with obstructive sleep apnea hypopnea syndrome," Tohoku J. Exp. Med., vol. 206, pp. 353360, Aug. 2005.

[42] D. C. Mack, J. T. Patrie, P. M. Suratt, R. A. Felder and M. Alwan, "Development and Preliminary Validation of Heart Rate and Breathing Rate Detection Using a Passive, Ballistocardiography-Based Sleep Monitoring System," IEEE Trans. Inf. Technol. Biomed., vol. 13, pp. 111-120, Jan. 2009.

[43] M. Brink, C. H. Müller and C. Schierz, "Contact-free measurement of heart rate, respiration rate, and body movements during sleep," Behav. Res. Methods, vol. 38, pp. 511-521, Aug. 2006.

[44] G. S. Chung, B. H. Choi, D. U. Jeong and K. S. Park, "Noninvasive heart rate variability analysis using loadcell-installed bed during sleep," in Proc. 29th Annu. Int. Conf. IEEE EMBS, 2007, pp. 2357-2360.

[45] J. Alihanka, K. Vaahtoranta and I. Saarikivi, "A new method for long-term monitoring of the ballistocardiogram, heart rate, and respiration," Am. J. Physiol., vol. 240, pp. R384-R392, May. 1981.

[46] E. Rauhala, M. Erkinjuntti and O. Polo, "Detection of periodic leg movements with a static-charge-sensitive bed," J. Sleep Res., vol. 5, pp. 246-250, Dec. 1996.

[47] K. Watanabe, et al, "Noninvasive measurement of heartbeat, respiration, snoring and body movements of a subject in bed via a pneumatic method," IEEE Trans. Biomed. Eng., vol. 52, pp. 2100-2107, Dec. 2005.

[48] N. A. Fox, C. Heneghan, M. Gonzalez, R. B. Shouldice and P. de Chazal, "An evaluation of a non-contact biomotion sensor with actimetry," in Proc. 29th Annu. Int. Conf. IEEE EMBS, 2007, pp. 2664-2668.

[49] Z. T. Beattie, C. C. Hagen, M. Pavel and T. L. Hayes, "Classification of breathing events using load cells under the bed," in Proc. 31st Annu. Int. Conf. IEEE EMBS, 2009, pp. 3921-3924. 
[50] N. Foubert, "Posture recognition and postural transition detection using bed-based pressure sensor arrays," Ma. Sc Thesis, Dept. of Sys. and Comp. Eng., Carleton University, Ottowa, Canada, 2010.

[51] M. Holtzman, et al, "Force estimation with a non-uniform pressure sensor array," in Proc. 25th Annu. Int. Conf. IEEE IMTC, 2008, pp. 1974-1979.

[52] M. H. Jones, R. Goubran and F. Knoefel, "Identifying movement onset times for a bed-based pressure sensor array," in Proc. IEEE Int. Workshop Med. Meas. Appl. 2006, pp. 111-114.

[53] I. Sato and M. Nakajima, "Non-contact breath motion monitoring system in full automation," in Proc. 27th Annu. Int. Conf. IEEE EMBS, 2005, pp. 3448-3451.

[54] R. Murthy, I. Pavlidis and P. Tsiamyrtzis, "Touchless monitoring of breathing function," in Proc. 26th Annu. Int. Conf. IEEE EMBS, 2004, pp. 1196-1199.

[55] A. $\mathrm{K}$. Ng, et al, "Could formant frequencies of snore signals be an alternative means for the diagnosis of obstructive sleep apnea?" Sleep Med., vol. 9, pp. 894-898, Dec. 2008.

[56] E. J. W. van Someren, "Actigraphic monitoring of movement and rest-activity rhythms in aging, alzheimer's disease and parkinson's disease," in Proc. 18th Annu. Int. Conf. IEEE EMBS, 1996, pp. 69-70 vol.1.

[57] A. Bates, M. J. Ling, J. Mann and D. K. Arvind, "Respiratory rate and flow waveform estimation from tri-axial accelerometer data," in Proc. Int. Conf. Body Sensor Networks, 2010, pp. 144-150.

[58] D. S. Morillo, J. L. R. Ojeda, L. F. C. Foix and A. L. Jimenez, "An AccelerometerBased Device for Sleep Apnea Screening," IEEE Trans. Inf. Technol. Biomed., vol. 14, pp. 491, Mar. 2010.

[59] A. Sadeh, "The role and validity of actigraphy in sleep medicine: An update," Sleep Medicine Reviews, vol. 15, pp. 259-267, 8. 2011.

[60] K. E. Bloch, "Polysomnography: a systematic review," Technol. Health Care, vol. 5, pp. 285-305, Oct. 1997.

[61] J. Pagani, et al, "Detection of central and obstructive sleep apnea in children using pulse transit time," Computers in Cardiology, pp. 529-532, 2002.

[62] J. V. Marcos, et al, "Radial basis function classifiers to help in the diagnosis of the obstructive sleep apnoea syndrome from nocturnal oximetry," Med. Biol. Eng. Comput., vol. 46, pp. 323-332, Apr. 2008.

[63] A. H. Khandoker, J. Gubbi and M. Palaniswami, "Recognizing central and 
obstructive sleep apnea events from normal breathing events in ECG recordings," Computers in Cardiology, pp. 681-684, 2008.

[64] A. H. Khandoker, M. Palaniswami and C. K. Karmakar, "Support Vector Machines for Automated Recognition of Obstructive Sleep Apnea Syndrome From ECG Recordings," IEEE Trans. Inf. Technol. Biomed., vol. 13, pp. 37-48, Jan. 2009.

[65] P. de Chazal, et al, "Automated processing of the single-lead electrocardiogram for the detection of obstructive sleep apnoea," IEEE Trans. Biomed. Eng., vol. 50, pp. 686696, Jun. 2003.

[66] F. Yen, K. Behbehani, E. A. Lucas, J. R. Burk and J. R. Axe, "A noninvasive technique for detecting obstructive and central sleep apnea," IEEE Trans. Biomed. Eng., vol. 44, pp. 1262-1268, Dec. 1997.

[67] E. Kaniusas, H. Pfutzner and B. Saletu, "Acoustical signal properties for cardiac/respiratory activity and apneas," IEEE Trans. Biomed. Eng., vol. 52, pp. 18121822, Nov. 2005.

[68] B. Schilit, N. Adams and R. Want, "Context-aware computing applications," in Proc. First Workshop Mobile Computing Sys. Appl. 1994, pp. 85-90.

[69] R. J. Robles and T. Kim, "Context aware systems, methods and trends in smart home technology," in Security-Enriched Urban Computing and Smart Grid, vol. 78, T. Kim, A. Stoica and R. Chang, Eds. Springer Berlin Heidelberg, 2010, pp. 149-158.

[70] Y. M. Kuo, J. S. Lee and P. C. Chung, "A visual context-awareness-based sleepingrespiration measurement system," IEEE Trans. Inf. Technol. Biomed., vol. 14, pp. 255265, Mar. 2010.

[71] W. Karlen and D. Floreano, "Adaptive Sleep-Wake Discrimination for Wearable Devices," IEEE Trans. Biomed. Eng., vol. 58, pp. 920, Apr. 2011.

[72] M. Jahn, et al, "The energy aware smart home," in Proc. 5th Int. Conf. Future Inf. Technology (FutureTech), 2010, pp. 1-8.

[73] W. Chen, X. Zhu and T. Nemoto, "A new sensory device and optimal position for monitoring HR/RR during sleep," in Proc. World Congress on Med. Physics and Biomed. Eng., Vol. 25 Iss. 7, 2009, pp. 126-129.

[74] Y. Tanimoto, H. Takechi, A. Tokuhiro and H. Yamamoto, "Measurements of pressure and sinking depth for mattress selection [spinal cord injured patients]," IEEE Trans. Instrum. Meas., vol. 52, pp. 1320-1324, 2003.

[75] M. Howell Jones, "Autonomous patient monitoring with a pressure sensor array," Ma. Sc. Thesis, Dept. Sys. and Comp. Eng, Carleton University, Ottawa, Canada, 2006. 
[76] J. Han, H. B. Shin, D. U. Jeong and K. S. Park, "Detection of apneic events from single channel nasal airflow using 2nd derivative method," Comput. Methods Programs Biomed., vol. 91, pp. 199-207, Sep. 2008.

[77] A. M. Adami, M. Pavel, T. L. Hayes and C. M. Singer, "Detection of Movement in Bed Using Unobtrusive Load Cell Sensors," IEEE Trans. Inf. Technol. Biomed., vol. 14, pp. 481-490, 2010.

[78] T. Harada, T. Sato and T. Mori, "Estimation of bed-ridden human's gross and slight movement based on pressure sensors distribution bed," in Proc. 2002 Int. Conf. Robotics and Automation, 2002, pp. 3795-3800.

[79] J. H. Shin, Y. J. Chee, D. U. Jeong and K. S. Park, "Nonconstrained Sleep Monitoring System and Algorithms Using Air-Mattress With Balancing Tube Method," IEEE Trans. Inf. Technol. Biomed., vol. 14, pp. 147-156, 2010.

[80] P. E. Shrout and J. L. Fleiss, "Intraclass correlations: uses in assessing rater reliability," Psychol. Bull., vol. 86, pp. 420-428, Mar. 1979.

[81] A. J. Myles, R. N. Feudale, Y. Liu, N. A. Woody and S. D. Brown, "An introduction to decision tree modeling," Journal of Chemometrics, vol. 18, pp. 275-285, 2004.

[82] L. Walsh, S. McLoone, J. Behan and T. Dishongh, "The deployment of a nonintrusive alternative to sleep/wake wrist actigraphy in a home-based study of the elderly," in Proc. 30th Annu. Int. Conf. IEEE EMBS, 2008, pp. 1687-1690.

[83] S. Nosal, et al, "Age-dependent changes of airway obstruction parameters," Journal of Physiology and Parmacology, vol. 58, Suppl 5, pp. 493-500, 2007.

[84] P. Varady, S. Bongar and Z. Benyo, "Detection of airway obstructions and sleep apnea by analyzing the phase relation of respiration movement signals," IEEE Trans. Instrum. Meas., vol. 52, pp. 2-6, Feb. 2003.

[85] J. R. Boulanger and C. Deroussent, "Preliminary based service evaluation for elderly people and healthcare professionals in residential home care units," in Proc. 2nd Int. Conf. Digital Society, 2008, pp. 93-101.

[86] G. Demiris, D. P. Oliver, G. Dickey, M. Skubic and M. Rantz, "Findings from a participatory evaluation of a smart home application for older adults," Technol. Health Care, vol. 16, pp. 111-118, 2008.

[87] J. Coughlin, L. A. D'Ambrosio, B. Reimer and M. R. Pratt, "Older adult perceptions of smart home technologies: Implications for research, policy \& market innovations in healthcare," in Proc. 29th Annu. Int. Conf. IEEE EMBS, 2007, pp. 1810-1815.

[88] K. Wild, L. Boise, J. Lundell and A. Foucek, "Unobtrusive In-Home Monitoring of 
Cognitive and Physical Health: Reactions and Perceptions of Older Adults," $J$ Appl Gerontol, vol. 27, pp. 181-200, 2008.

[89] J. S. Beaudin, S. S. Intille and M. E. Morris, "To track or not to track: user reactions to concepts in longitudinal health monitoring," J. Med. Internet Res., vol. 8, pp. e29, 2006.

[90] A. Mihailidis, A. Cockburn, C. Longley and J. Boger, "The acceptability of home monitoring technology among community-dwelling older adults and baby boomers," Assist. Technol., vol. 20, pp. 1-12, Spring. 2008.

[91] H. W. Tyrer, et al, "Technology for successful aging," in Proc. 28th Annu. Int. Conf. IEEE EMBS, 2006, pp. 3290-3293.

[92] R. Steele, C. Secombe and W. Brookes, "Using wireless sensor networks for aged care: The patient's perspective," in Proc. Pervasive Health Conference and Workshops, 2006, pp. 1-10.

[93] G. Demiris, et al, "Older adults' attitudes towards and perceptions of "smart home" technologies: a pilot study," Med. Inform. Internet Med., vol. 29, pp. 87-94, Jun. 2004.

[94] G. Demiris, M. J. Rantz, M. Skubic, M. A. Aud and H. W. Tyrer Jr, "Home-based assistive technologies for elderly: Attitudes and perceptions," in Proc. AMIA Annual Symposium, 2005, pp. 935.

[95] G. Demiris, B. K. Hensel, M. Skubic and M. Rantz, "Senior residents' perceived need of and preferences for "smart home" sensor technologies," Int. J. Technol. Assess. Health Care, vol. 24, pp. 120-124, Winter. 2008.

[96] R. Steele, A. Lo, C. Secombe and Y. K. Wong, "Elderly persons' perception and acceptance of using wireless sensor networks to assist healthcare," Int. J. Med. Inform., vol. 78, pp. 788-801, 2009.

[97] M. Govercin et al., "Defining the user requirements for wearable and optical fall prediction and fall detection devices for home use," Inform. Health Social Care, vol. 35, pp. 177-187, Sep-Dec. 2010.

[98] K. L. Courtney, G. Demiris, M. Rantz and M. Skubic, "Needing smart home technologies: the perspectives of older adults in continuing care retirement communities," Inform. Prim. Care., vol. 16, pp. 195-201, 2008.

[99] T. Bratan, M. Clarke, R. Jones, A. Larkworthy and R. Paul, "Evaluation of the practical feasibility and acceptability of home monitoring in residential homes," $J$. Telemed. Telecare, vol. 11, Suppl 1, pp. 29-31, 2005.

[100] M. Marschollek et al., "Multimodal home monitoring of elderly people--first results 
from the LASS study," in Proc. 21st Int. Conf. Adv. Inf. Networking Appl. Workshops, 2007, pp. 815-819.

[101] A. Melander-Wikman, M. Jansson, J. Hallberg, C. Mortberg and G. Gard, "The Lighthouse Alarm and Locator Trial - a pilot study," Technol. Health Care, vol. 15, pp. 203-212, 2007.

[102] K. L. Courtney, G. Demiris and B. K. Hensel, "Obtrusiveneness of informationbased assistive technologies as perceived by older adults in residential care facilities: a secondary analysis," Med Inform Internet Med, vol. 32, pp. 241-9, 2007.

[103] B. K. Hensel, G. Demiris and K. L. Courtney, "Defining Obtrusiveness in Home Telehealth Technologies: A Conceptual Framework," J Am Med Inform Assoc., vol. 13, pp. 428-31, 2006 Jul-Aug. 2006.

[104] L. Versweyveld, "Nurses and patients more than satisfied with Nova Scotia home telehealth pilot," Virtual Medical Worlds Monthly, Apr. 2002. 